\title{
Enantioselective Construction of Chiral Cyclopropa[c]coumarins via Lewis Base Catalyzed Cyclopropanation
}

\author{
Jun-Chao Sun ${ }^{a}$, Xiao-Hui Wang ${ }^{a}$, Cong-Bin $\mathrm{Ji}^{b}$, Yi-Yuan Peng ${ }^{a}$ and Xing-Ping Zeng ${ }^{a, *}$
}

${ }^{a}$ Key Laboratory of Small Functional Organic Molecule, Ministry of Education and Jiangxi Key Laboratory of Green Chemistry, College of Chemistry and Chemical Engineering, Jiangxi Normal University, Nanchang, Jiangxi 330022, China. E-mail: 005173@ jxnu.edu.cn (X.-P. Zeng)

${ }^{b}$ Jiangxi Provincial Research of Targeting Pharmaceutical Engineering Technology, Shangrao Normal University, Shangrao, Jiangxi, 334001, China

Contents

Page

1. Crystallographic Information for Product 11a \& $15 f$

2. NMR Spectrum

S5-S58

3. HLPC spectrum

S59-S84 


\section{Crystallographic Information for Product 11a \& $15 f$}

The single crystal of compound 11a \& $\mathbf{1 5 f}$ was prepared from its solution in dichloromethane/petroleum ether by slow evaporation of the solvent. The data integration and empirical absorption correction were carried out using SAINT program. Using Olex2 and SHELXTL, the structure was solved by direct method and refined matrix least-squares on F2 with anisotropic displacement. Non-hydrogen atoms were refined anisotropically, hydrogen atoms were constrained to ideal geometries.

The absolute configuration was determined by single crystal X-ray diffraction analysis on Rigaku XtaLAB PRO MM003-DS dual system with a $\mathrm{Cu}$ micro-focus source.

\subsection{Single-crystal X-ray crystallography of 11a}

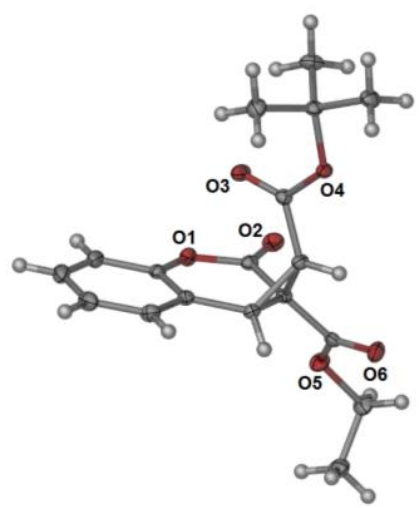

Figure S1. ORTEP of 11a (The ellipsoid contour of probability level is 50\%).

Table S1 Crystal data and structure refinement for 11a.

\begin{tabular}{ll}
\hline Identification code & exp_951 \\
Empirical formula & $\mathrm{C}_{18} \mathrm{H}_{20} \mathrm{O}_{6}$ \\
Formula weight & 332.34 \\
Temperature/K & $100.00(10)$ \\
Crystal system & orthorhombic \\
Space group & $\mathrm{P} 2{ }_{1} 2_{1} 2_{1}$ \\
$\mathrm{a} / \AA$ & $5.81970(10)$ \\
$\mathrm{b} / \AA$ & $16.03060(10)$ \\
$\mathrm{c} / \AA$ & $17.9580(2)$ \\
$\alpha /{ }^{\circ}$ & 90 \\
$\beta /{ }^{\circ}$ & 90 \\
$\gamma /{ }^{\circ}$ & 90 \\
Volume/ $\AA^{3}$ & $1675.36(4)$ \\
$\mathrm{Z}$ & 4 \\
$\rho_{\text {calcg }} / \mathrm{cm}^{3}$ & 1.318 \\
$\mu / \mathrm{mm}^{-1}$ & 0.825 \\
$\mathrm{~F}(000)$ & 704.0 \\
$\mathrm{Crystal}$ size $/ \mathrm{mm}^{3}$ & $0.32 \times 0.26 \times 0.22$ \\
$\mathrm{Radiation}$ & $\mathrm{CuK \alpha}(\lambda=1.54184)$ \\
$2 \Theta$ range for data collection $/{ }^{\circ}$ & 7.392 to 148.9 \\
\hline
\end{tabular}




\begin{tabular}{ll}
\hline Index ranges & $-7 \leq \mathrm{h} \leq 5,-19 \leq \mathrm{k} \leq 19,-22 \leq 1 \leq 22$ \\
Reflections collected & 16041 \\
Independent reflections & $3371\left[\mathrm{R}_{\text {int }}=0.0315, \mathrm{R}_{\text {sigma }}=0.0219\right]$ \\
Data/restraints/parameters & $3371 / 0 / 221$ \\
Goodness-of-fit on $\mathrm{F}^{2}$ & 1.053 \\
Final $\mathrm{R}$ indexes [I $>=2 \sigma(\mathrm{I})]$ & $\mathrm{R}_{1}=0.0260, \mathrm{wR}_{2}=0.0654$ \\
Final R indexes [all data] & $\mathrm{R}_{1}=0.0265, \mathrm{wR}_{2}=0.0657$ \\
Largest diff. peak/hole / e $\AA^{-3}$ & $0.14 /-0.20$ \\
Flack parameter & $0.04(6)$ \\
\hline
\end{tabular}

\subsection{Single-crystal X-ray crystallography of $15 f$.}

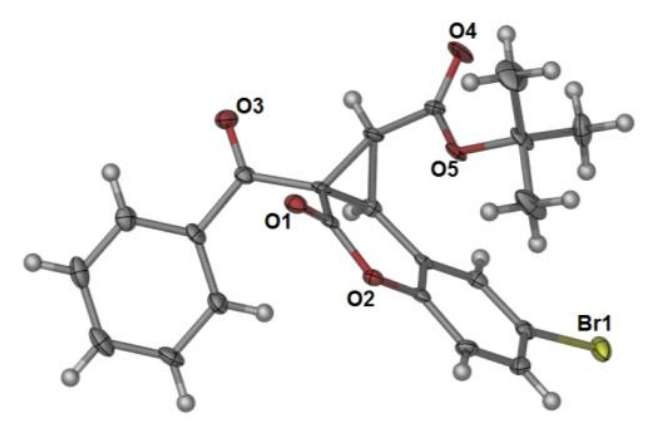

Figure S2. ORTEP of $\mathbf{1 5 f}$ (The ellipsoid contour of probability level is $50 \%$ ).

Table S2. Crystal data and structure refinement for $\mathbf{1 5 f}$.

\begin{tabular}{ll}
\hline Identification code & exp_1138 \\
Empirical formula & $\mathrm{C}_{22} \mathrm{H}_{19} \mathrm{BrO}_{5}$ \\
Formula weight & 443.28 \\
Temperature/K & 100 \\
Crystal system & orthorhombic \\
Space group & $\mathrm{P} 2{ }_{1} 2_{1} 2_{1}$ \\
a/A & $10.62270(10)$ \\
$\mathrm{b} / \AA$ & $12.13930(10)$ \\
$\mathrm{c} / \AA$ & $15.69910(10)$ \\
$\alpha /{ }^{\circ}$ & 90 \\
$\beta /{ }^{\circ}$ & 90 \\
$\gamma /{ }^{\circ}$ & 90 \\
Volume/ $\AA^{3}$ & $2024.43(3)$ \\
$\mathrm{Z}$ & 4 \\
$\rho_{\text {calcg/cm }}^{3}$ & 1.454 \\
$\mu / \mathrm{mm}^{-1}$ & 3.025 \\
$\mathrm{~F}(000)$ & 904.0 \\
Crystal size/mm & 3 \\
Radiation & $0.38 \times 0.28 \times 0.25$ \\
$2 \Theta$ range for data collection $/{ }^{\circ}$ & $\mathrm{CuK \alpha}(\lambda=1.54178)$ \\
Index ranges & 9.208 to 149.52 \\
\hline
\end{tabular}


Reflections collected

Independent reflections

Data/restraints/parameters

Goodness-of-fit on $\mathrm{F}^{2}$

Final $\mathrm{R}$ indexes $[\mathrm{I}>=2 \sigma(\mathrm{I})]$

Final $\mathrm{R}$ indexes [all data]

Largest diff. peak/hole / e $\AA^{-3}$

Flack parameter
48301

$4107\left[\mathrm{R}_{\mathrm{int}}=0.0989, \mathrm{R}_{\mathrm{sigma}}=0.0323\right]$

$4107 / 0 / 254$

1.035

$\mathrm{R}_{1}=0.0328, \mathrm{wR}_{2}=0.0849$

$\mathrm{R}_{1}=0.0331, \mathrm{wR}_{2}=0.0851$

$0.48 /-0.67$

$-0.001(14)$ 
${ }^{1} \mathrm{H}$ NMR Spectrum (400 MHz, Chloroform- $d$ ) of 11a sjc-g-76

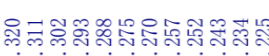
Hititititititi

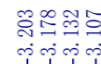

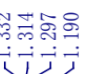

$\operatorname{coo}^{t} \mathrm{Bu}$

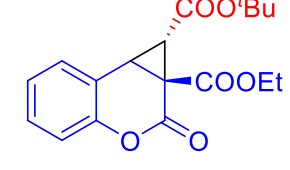

11a

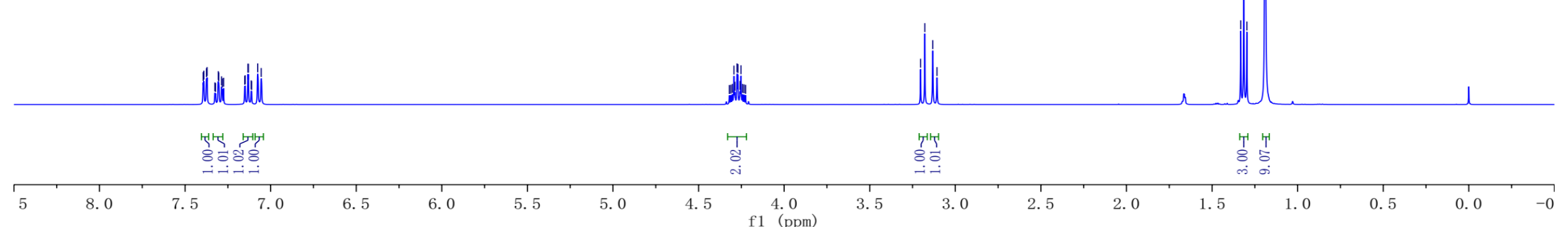


${ }^{13} \mathrm{C}\left\{{ }^{1} \mathrm{H}\right\}$ NMR Spectrum (101 MHz, Chloroform- $d$ ) of 11a

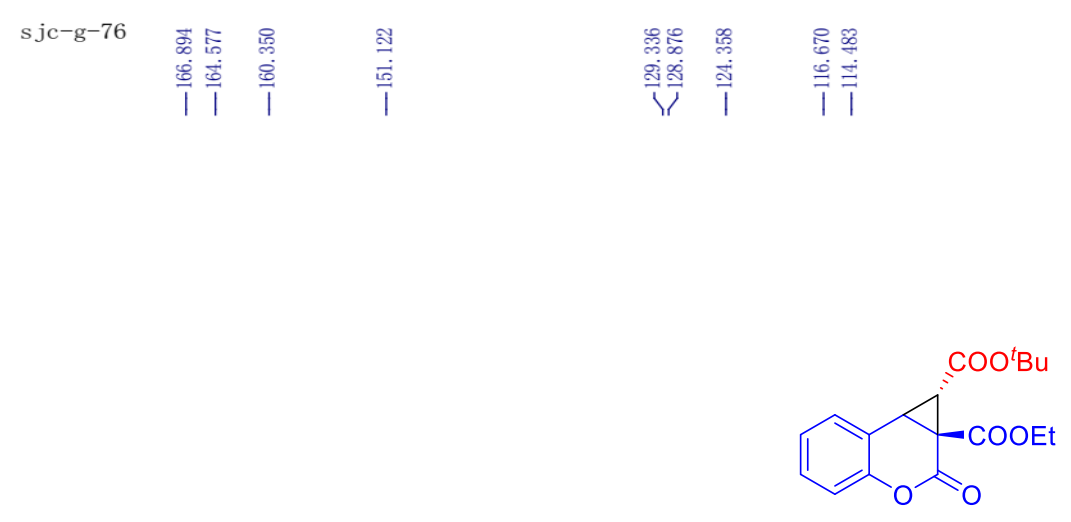

11a

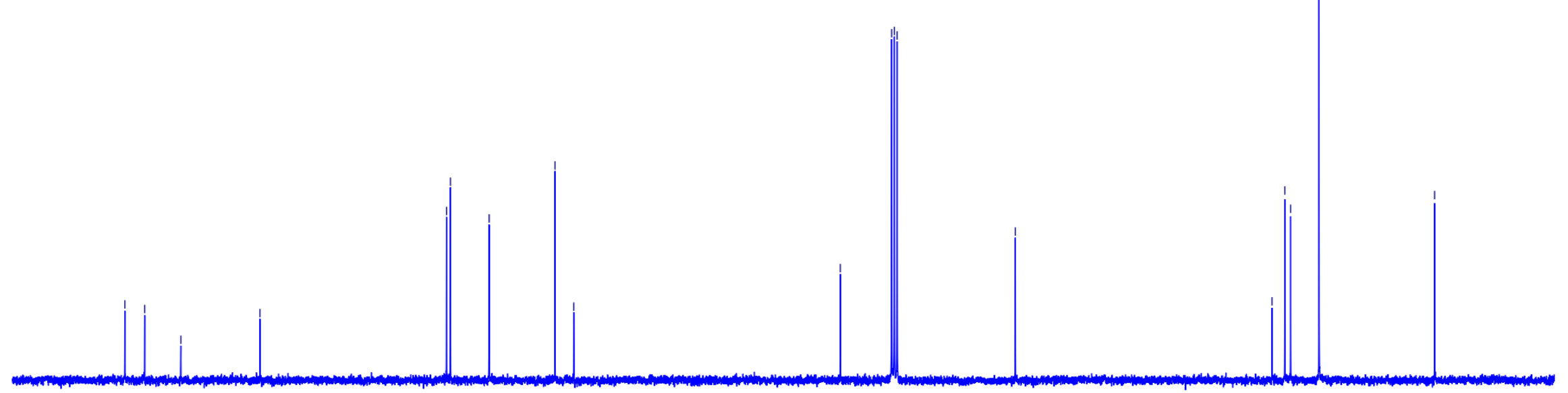

30

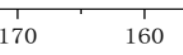

150

140
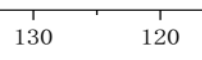

110

100

f1 $\stackrel{90}{(\mathrm{ppm})}$

80

70

60

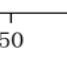

40

30 
${ }^{1} \mathrm{H}$ NMR Spectrum (400 MHz, Chloroform- $d$ ) of 11a

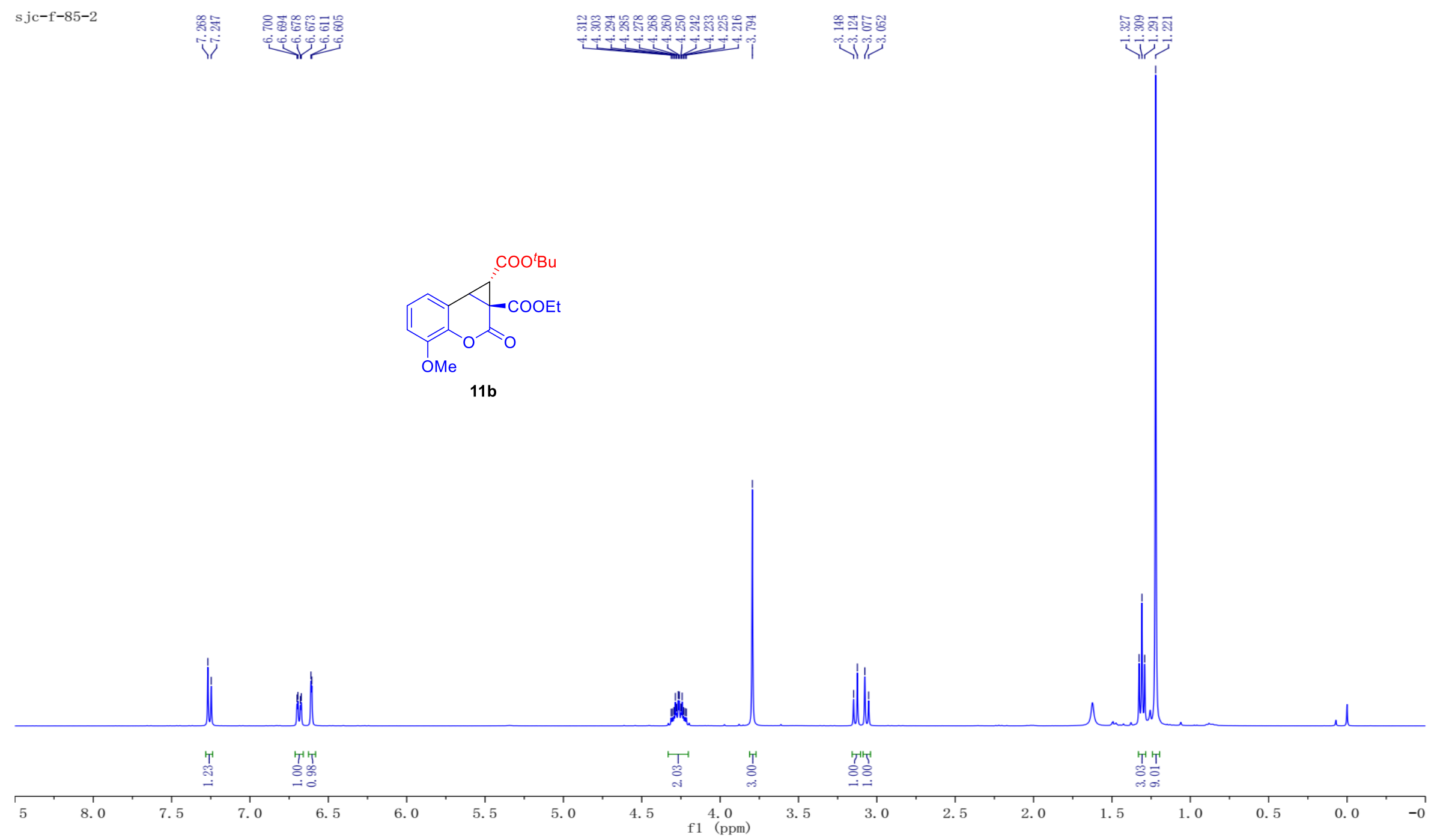


${ }^{13} \mathrm{C}\left\{{ }^{1} \mathrm{H}\right\}$ NMR Spectrum (101 MHz, Chloroform- $d$ ) of $\mathbf{1 1 b}$
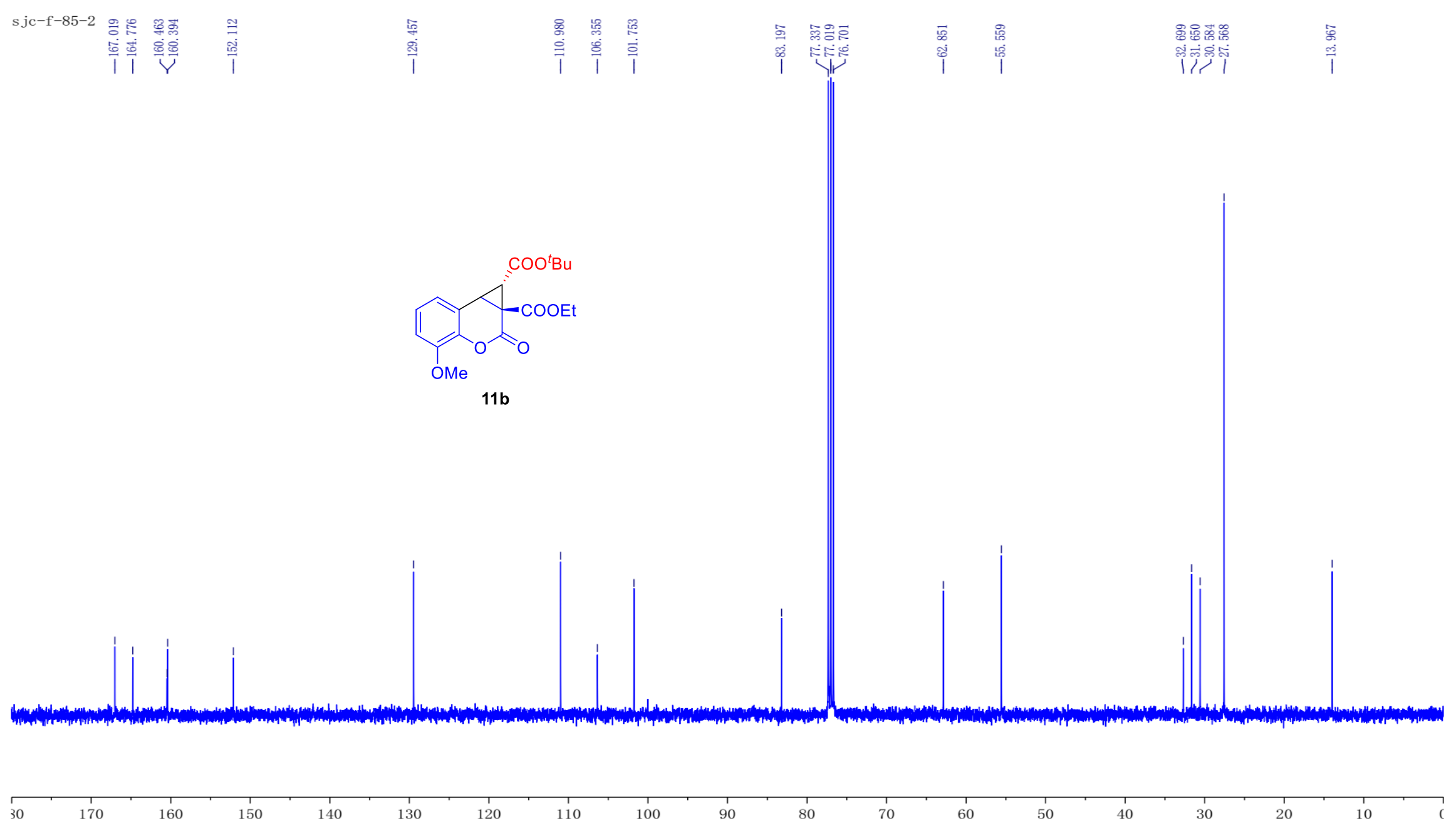
${ }^{1} \mathrm{H}$ NMR Spectrum (400 MHz, Chloroform- $d$ ) of 11c

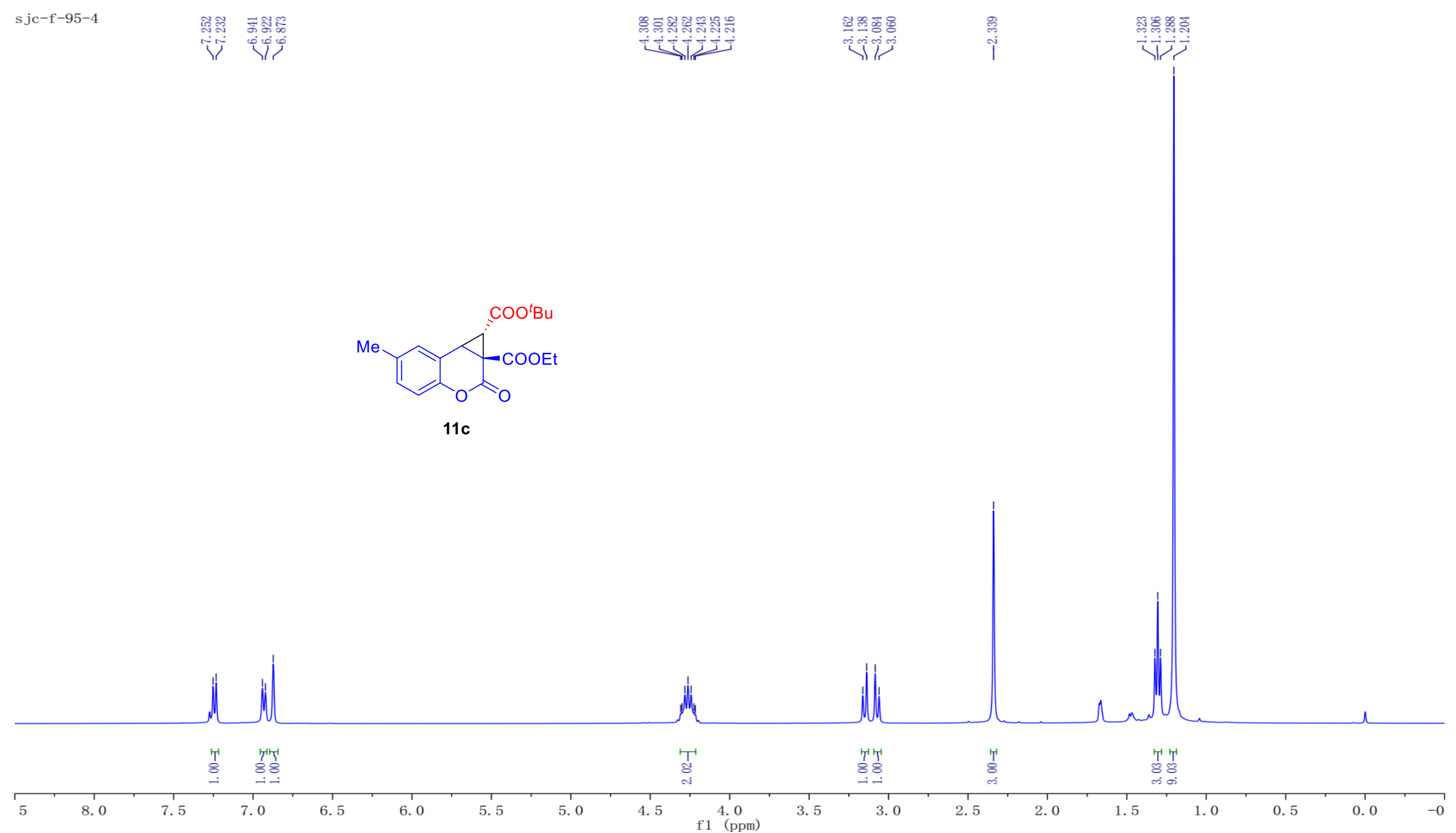


${ }^{13} \mathrm{C}\left\{{ }^{1} \mathrm{H}\right\}$ NMR Spectrum (101 MHz, Chloroform- $d$ ) of 11c

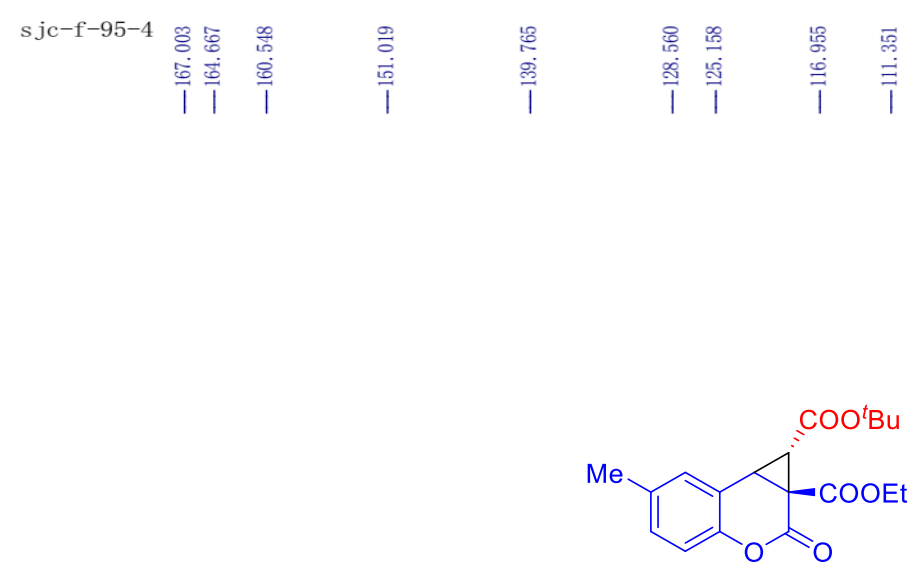

$11 \mathrm{c}$

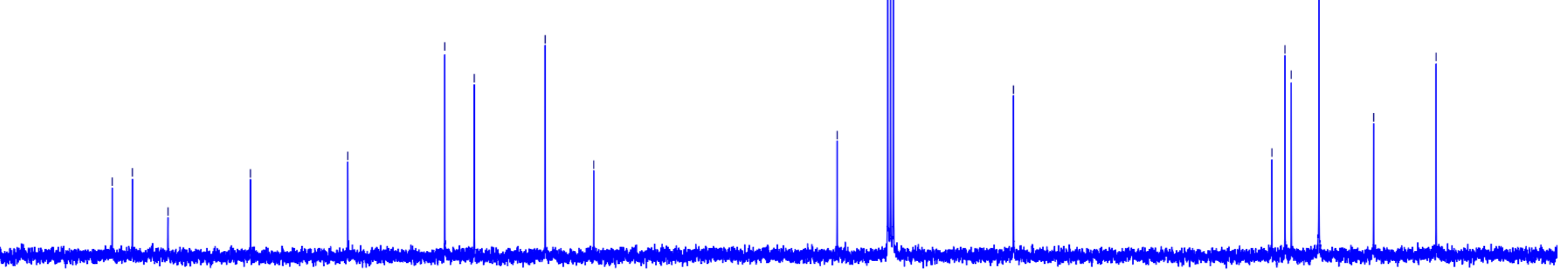

\section{0}
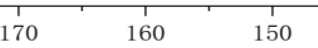

140
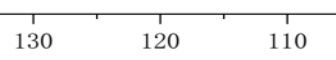

100

f1 $\stackrel{90}{(\mathrm{ppm})}$

80

70

60

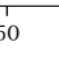

30 
${ }^{1} \mathrm{H}$ NMR Spectrum (400 MHz, Chloroform- $d$ ) of 11d

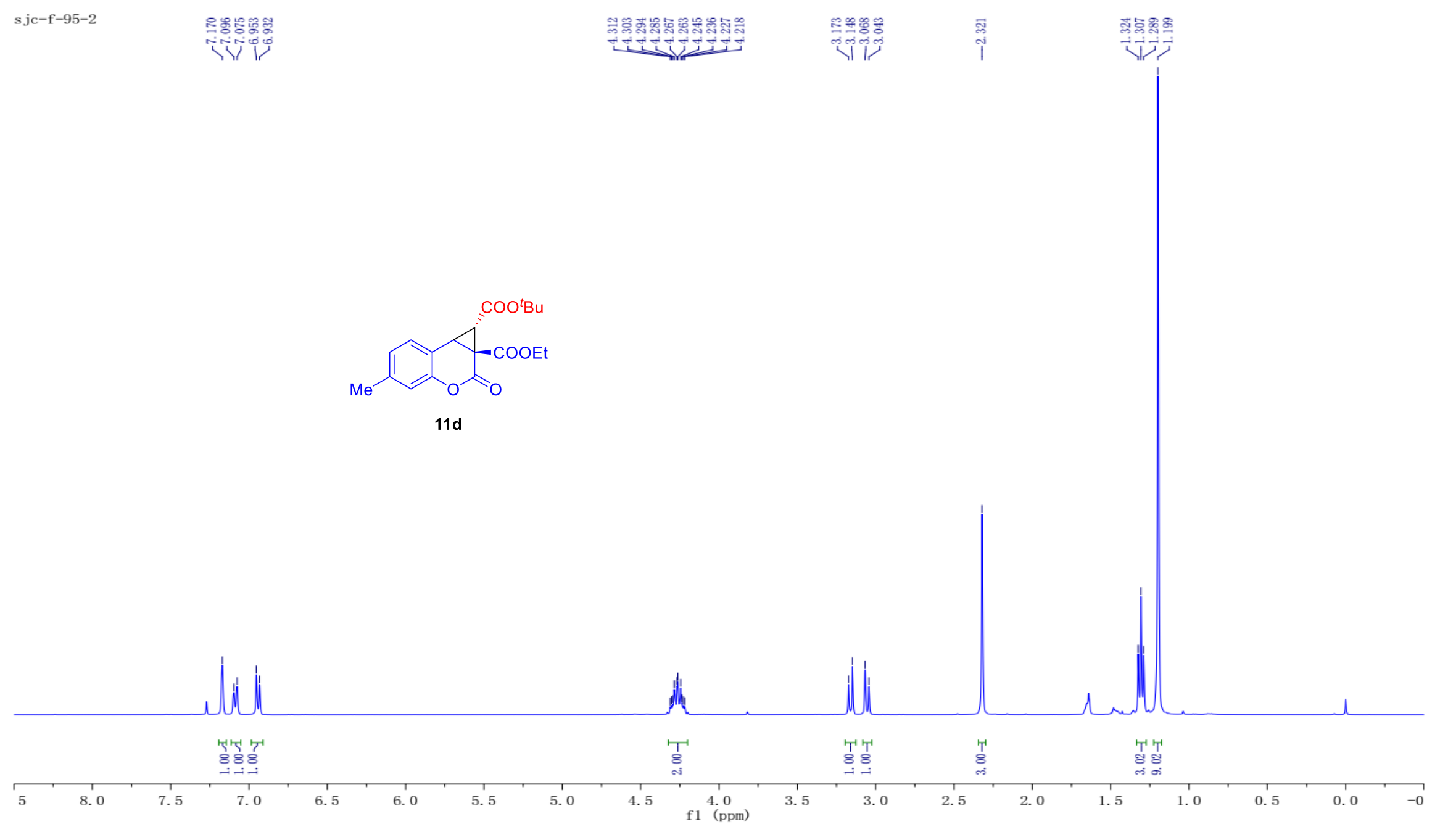


${ }^{13} \mathrm{C}\left\{{ }^{1} \mathrm{H}\right\}$ NMR Spectrum (101 MHz, Chloroform- $d$ ) of 11d
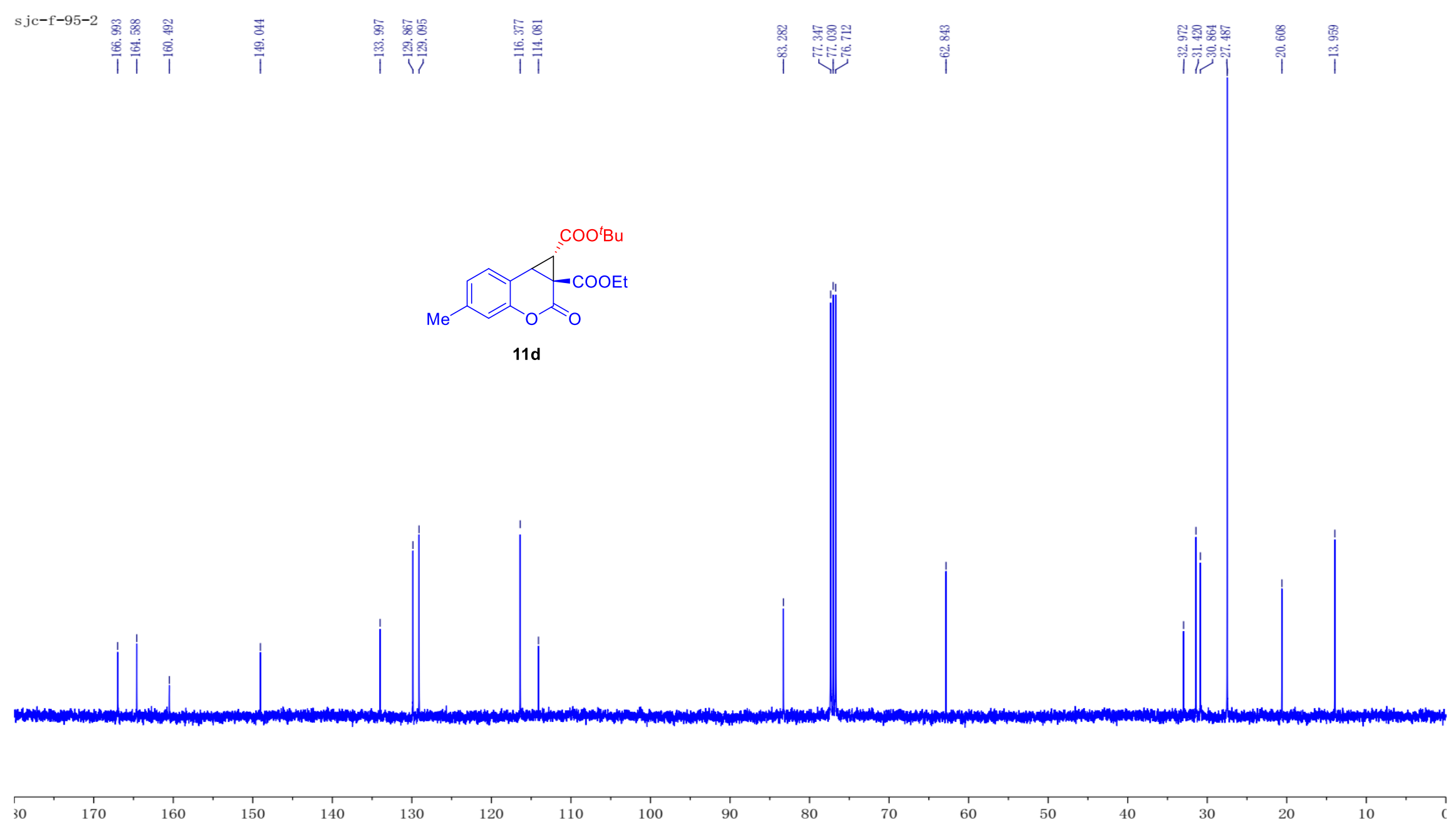
${ }^{1} \mathrm{H}$ NMR Spectrum (400 MHz, Chloroform- $d$ ) of 11e

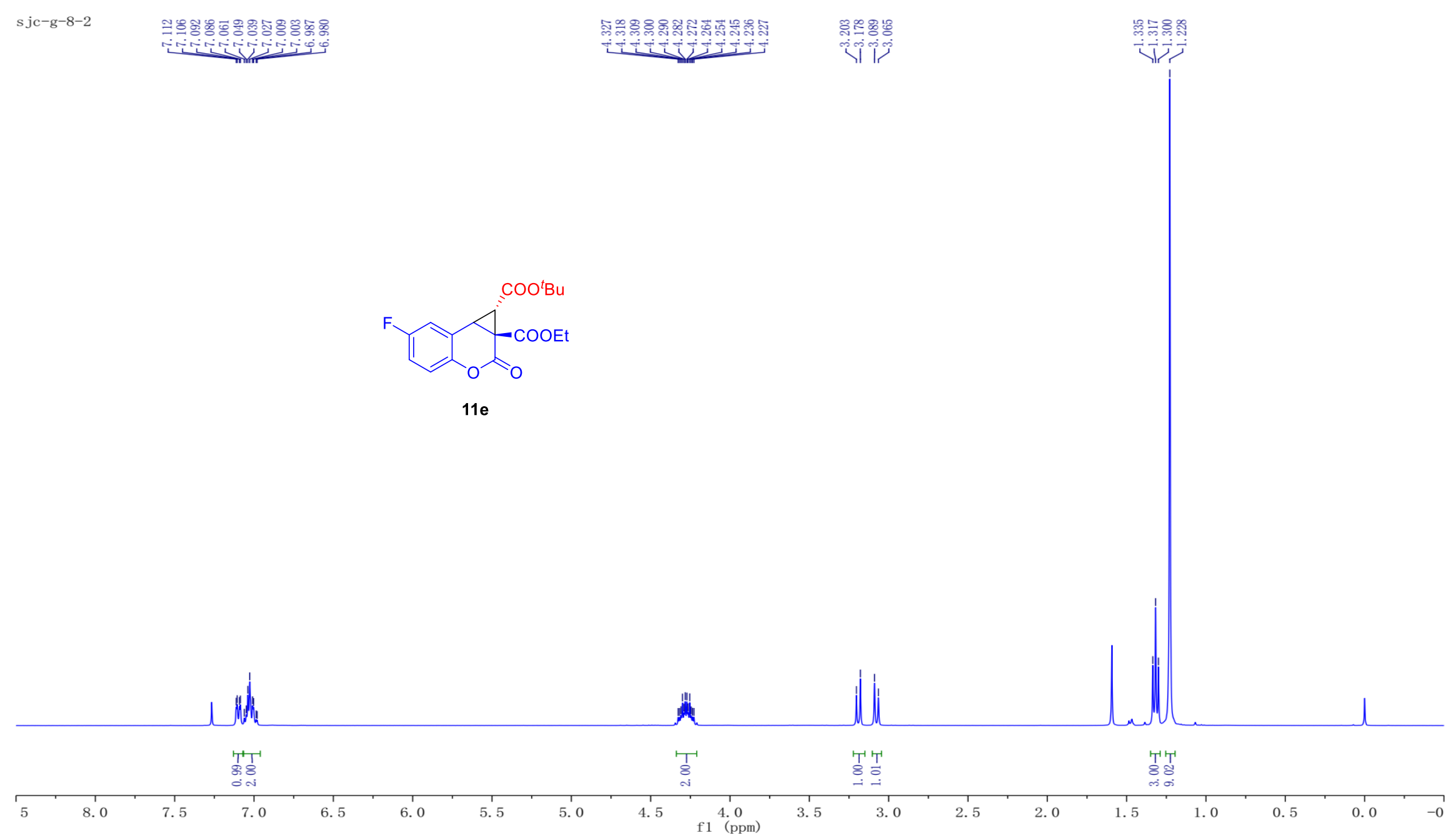


${ }^{13} \mathrm{C}\left\{{ }^{1} \mathrm{H}\right\}$ NMR Spectrum (101 MHz, Chloroform- $d$ ) of 11e

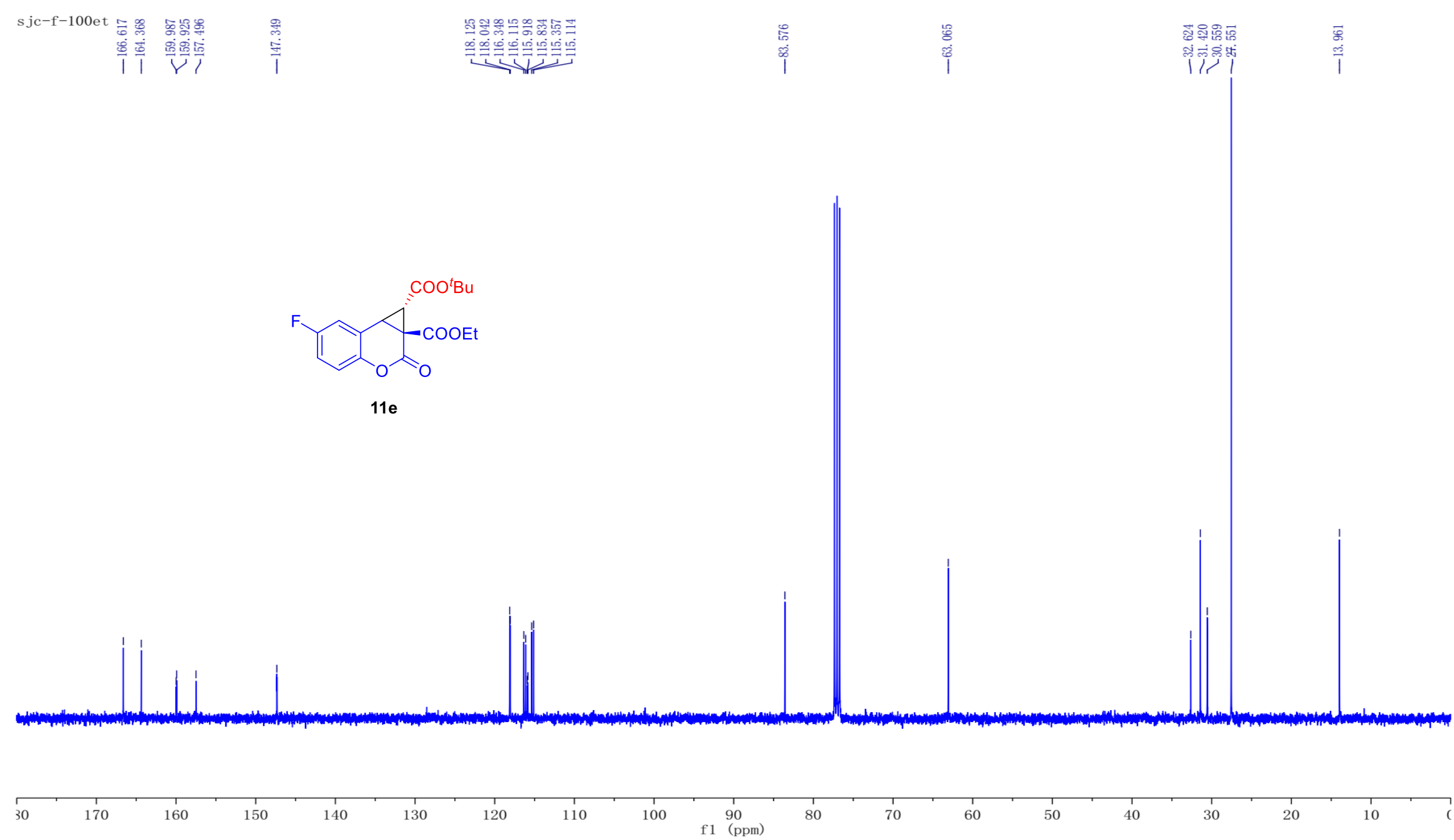


${ }^{19} \mathrm{~F}$ NMR (282 MHz, Chloroform- $d$ ) of 11e

sjc-f-100et

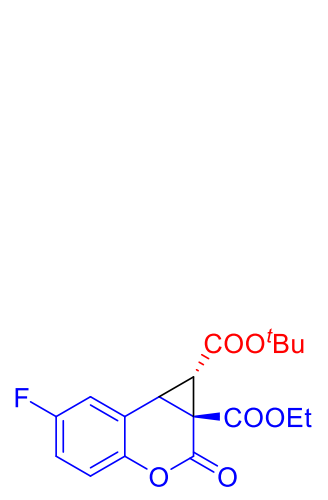

$11 \mathrm{e}$ 
${ }^{1} \mathrm{H}$ NMR Spectrum (400 MHz, Chloroform- $d$ ) of $\mathbf{1 1 f}$

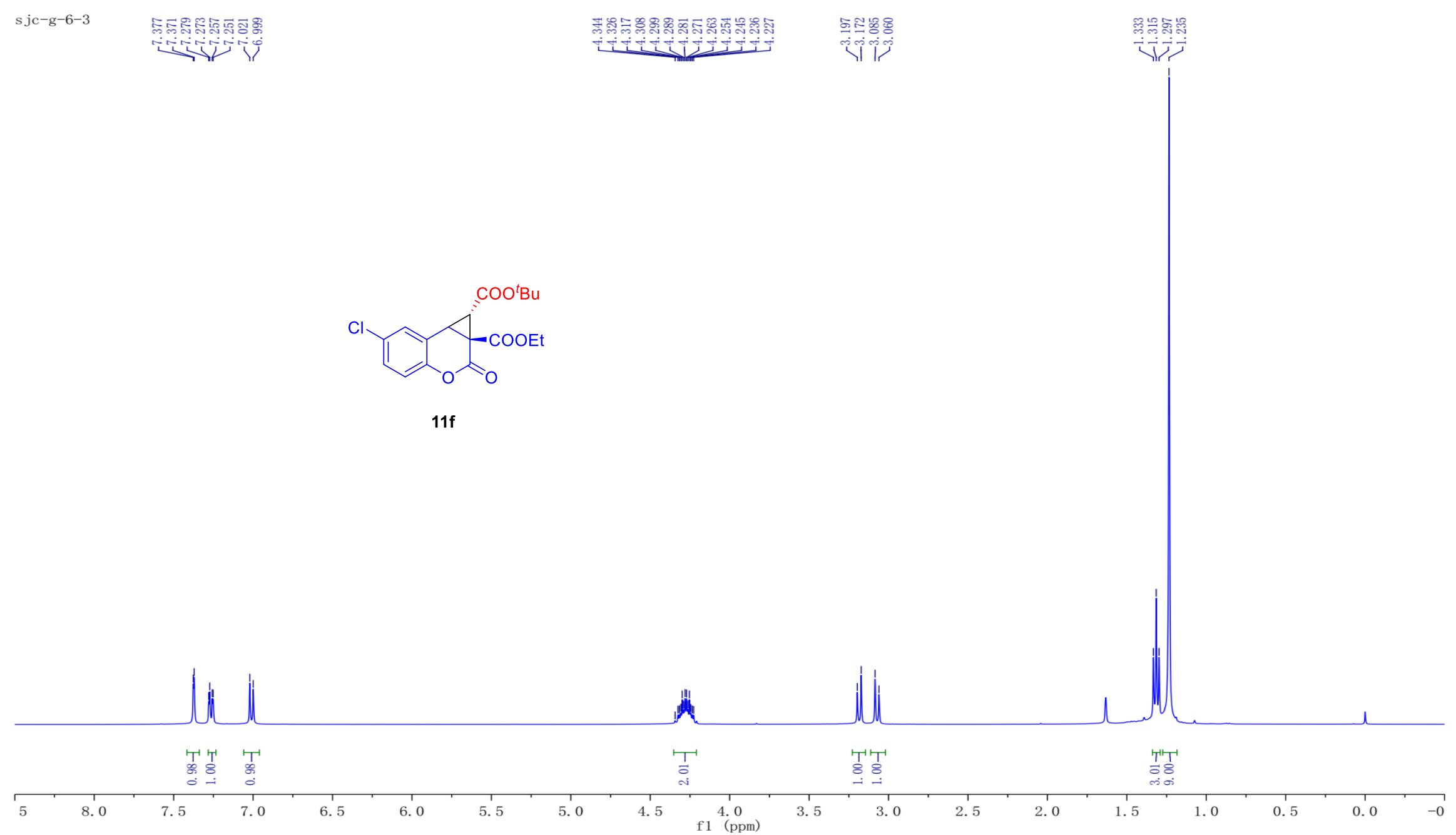


${ }^{13} \mathrm{C}\left\{{ }^{1} \mathrm{H}\right\}$ NMR Spectrum (101 MHz, Chloroform- $d$ ) of $\mathbf{1 1 f}$

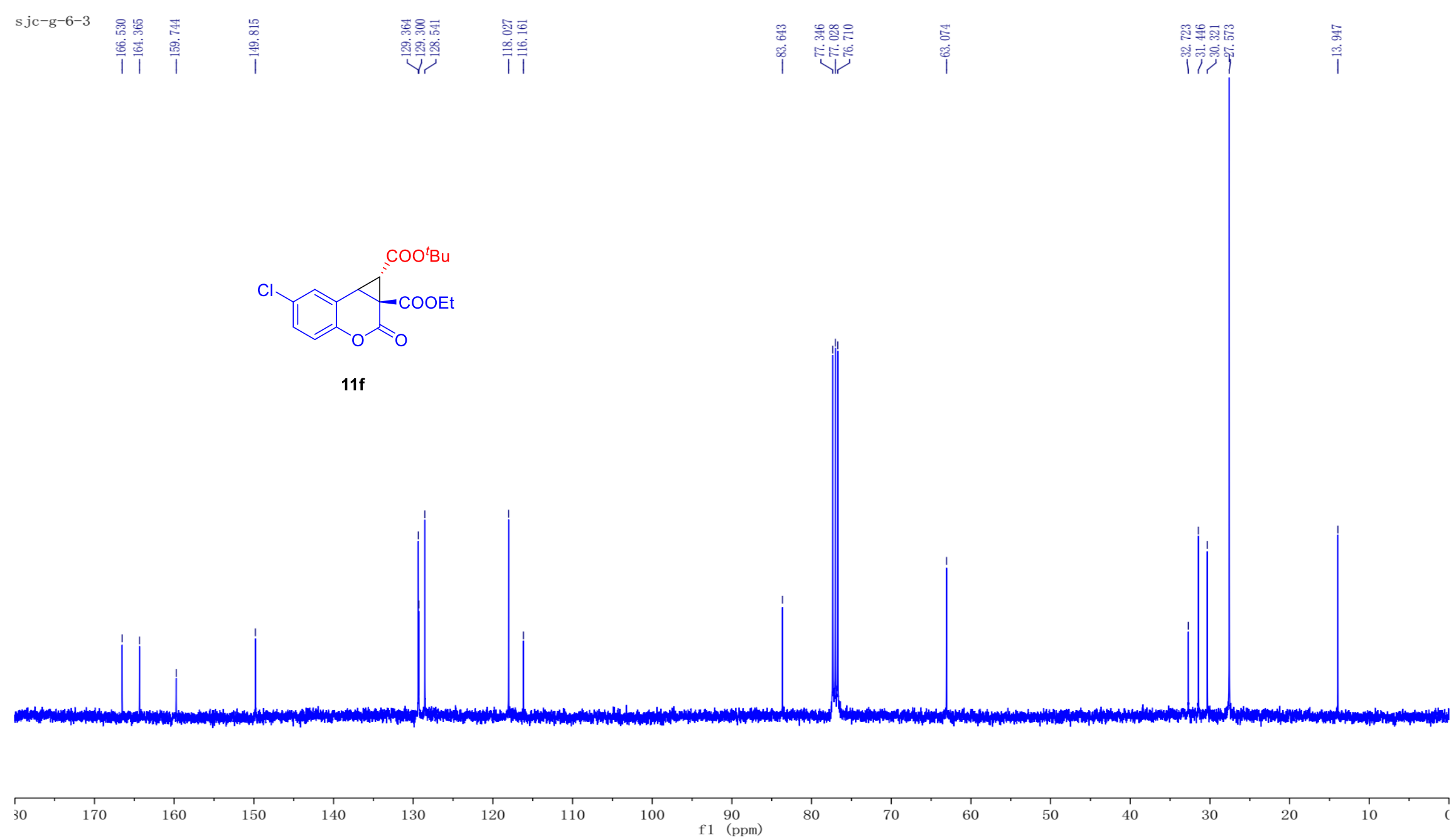


${ }^{1} \mathrm{H}$ NMR Spectrum (400 MHz, Chloroform- $d$ ) of $\mathbf{1 1 g}$

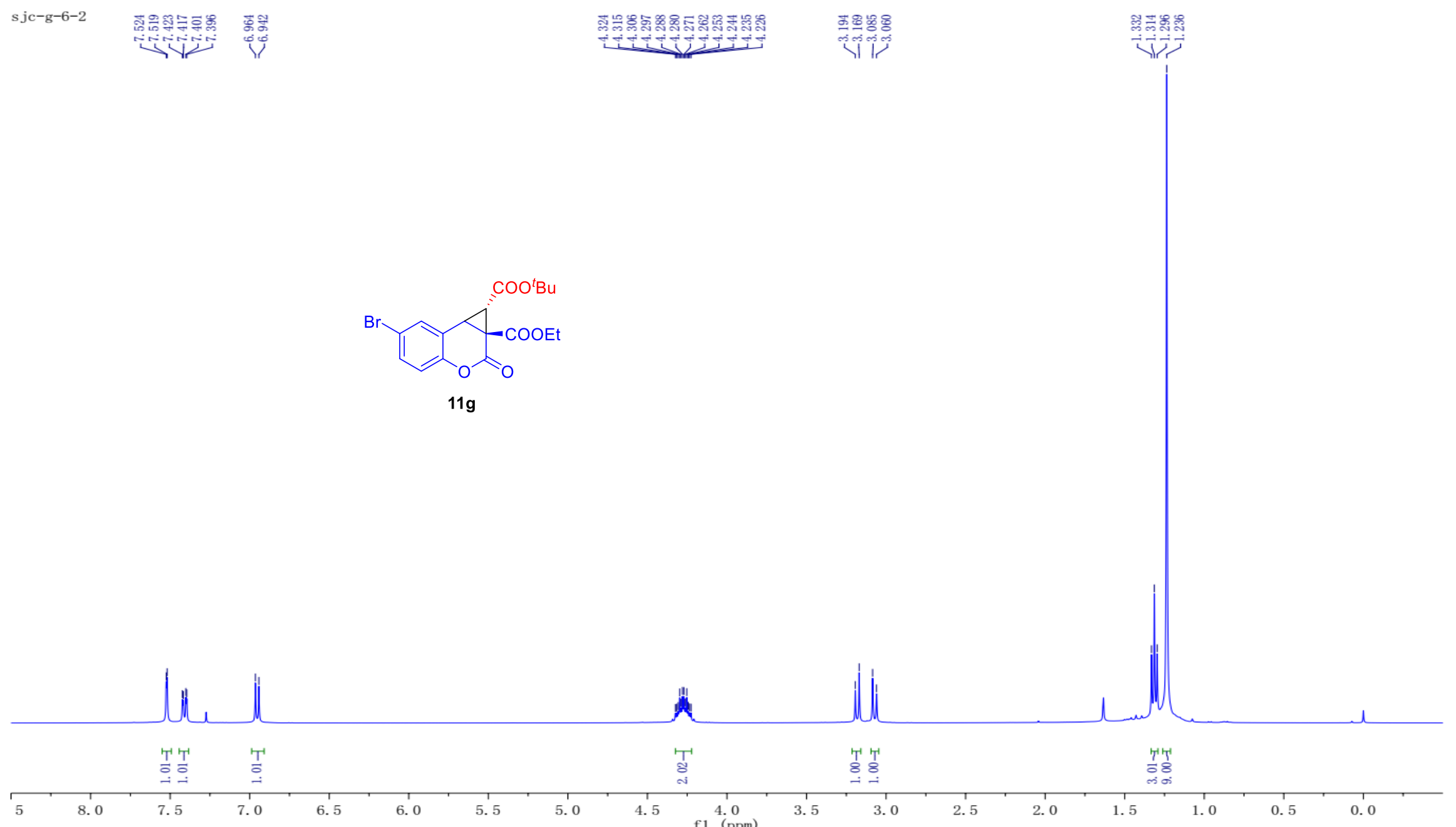


${ }^{13} \mathrm{C}\left\{{ }^{1} \mathrm{H}\right\}$ NMR Spectrum (101 MHz, Chloroform- $d$ ) of $\mathbf{1 1 g}$
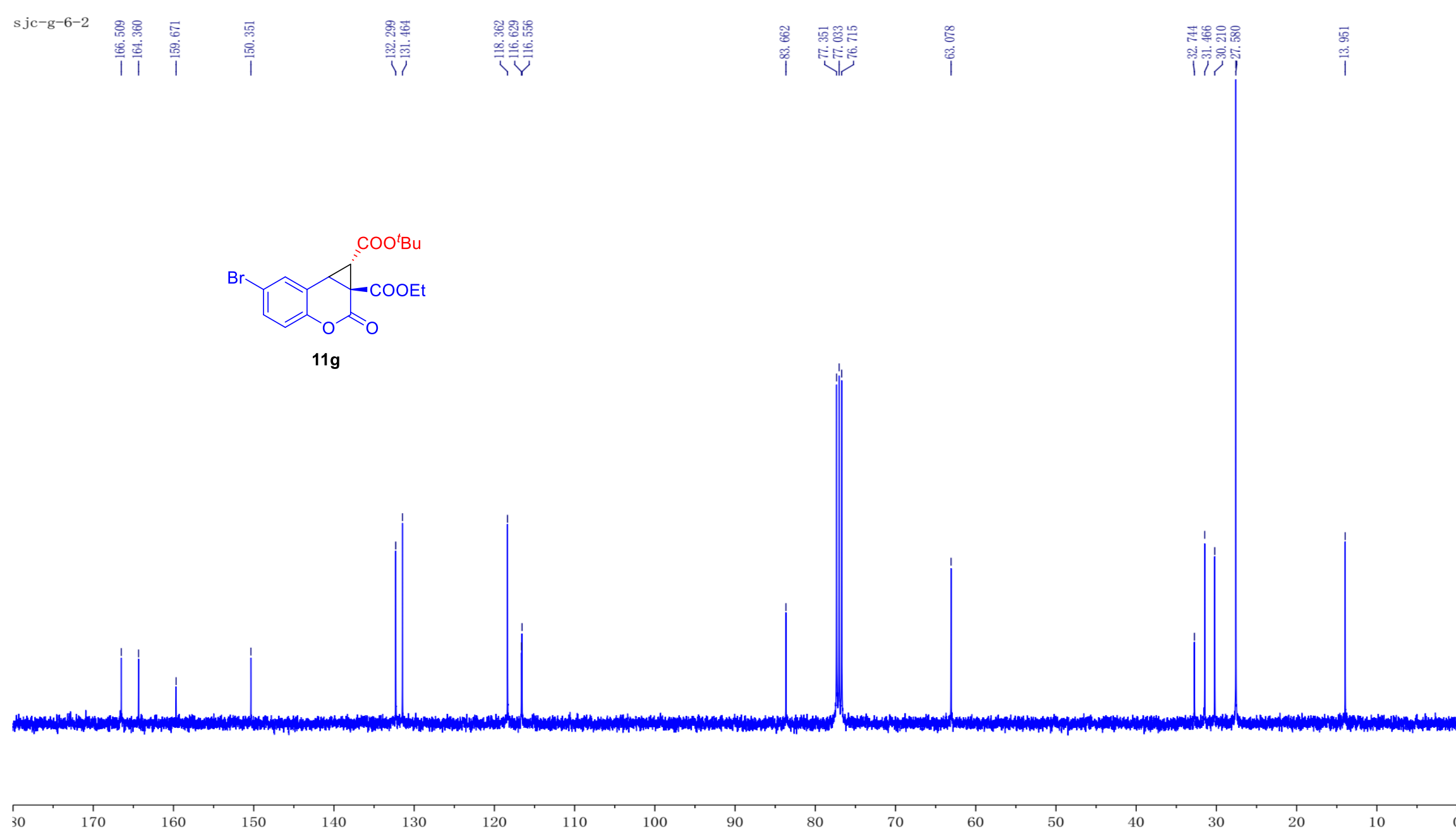
${ }^{1} \mathrm{H}$ NMR Spectrum (400 MHz, Chloroform- $d$ ) of $\mathbf{1 1 h}$
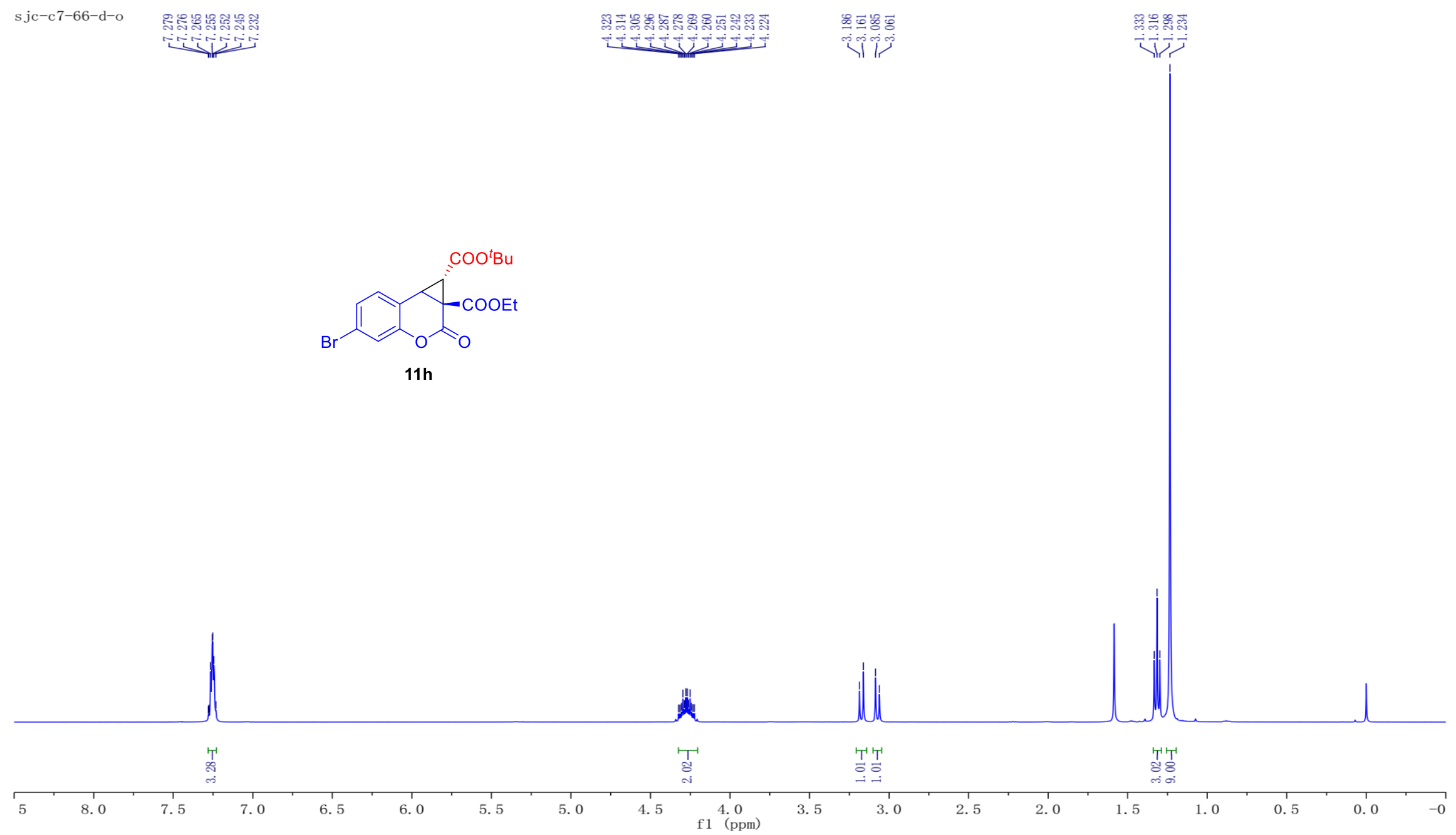
${ }^{13} \mathrm{C}\left\{{ }^{1} \mathrm{H}\right\}$ NMR Spectrum (101 MHz, Chloroform- $d$ ) of $\mathbf{1 1 h}$
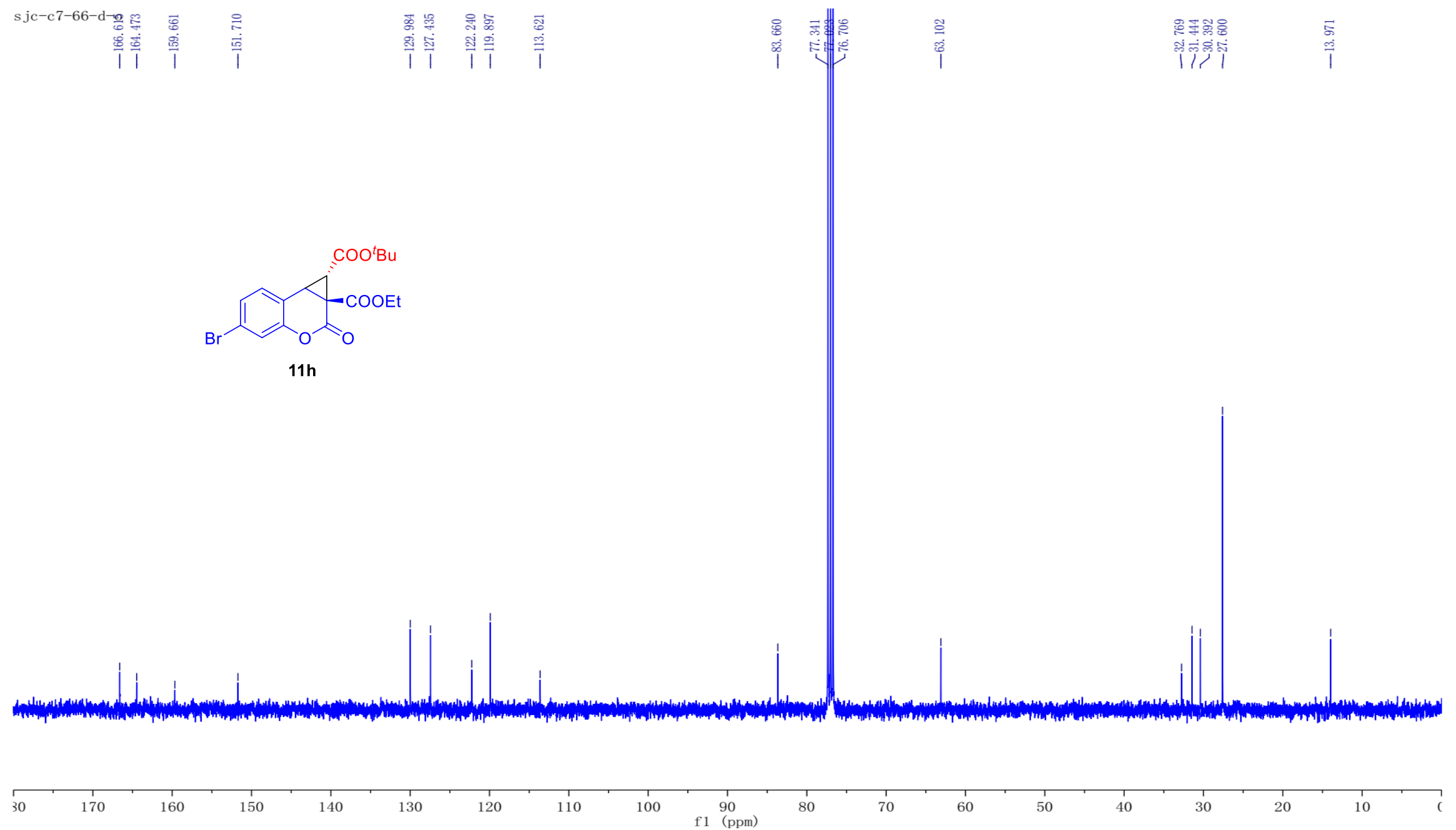
${ }^{1} \mathrm{H}$ NMR Spectrum (400 MHz, Chloroform- $d$ ) of 11i

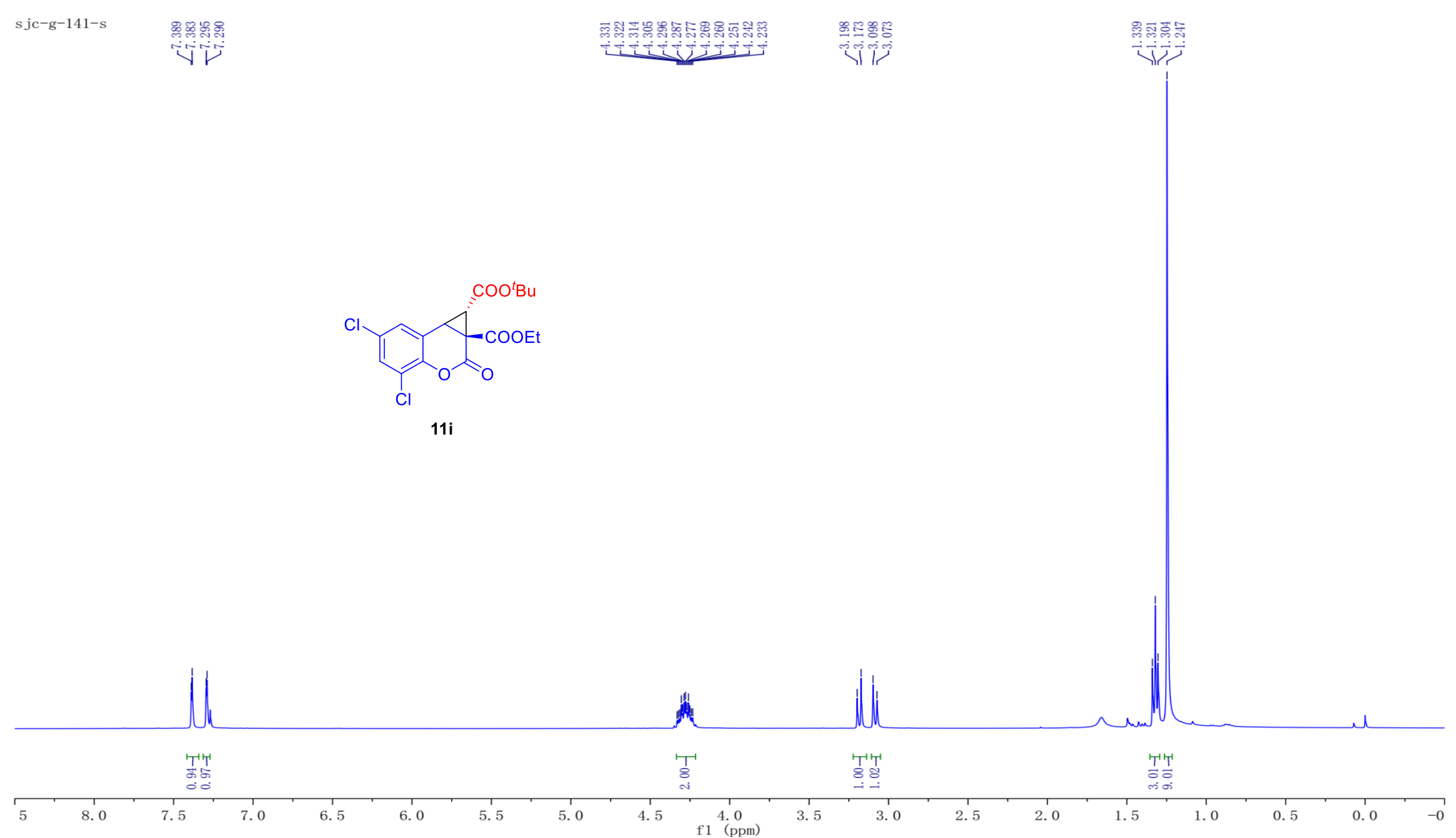


${ }^{13} \mathrm{C}\left\{{ }^{1} \mathrm{H}\right\}$ NMR Spectrum (101 MHz, Chloroform- $d$ ) of $\mathbf{1 1 i}$

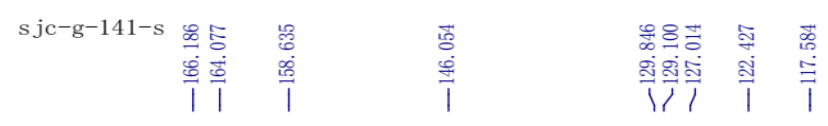

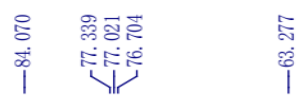

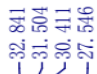
$\underset{\substack{F \\ \text { । }}}{\vec{j}}$

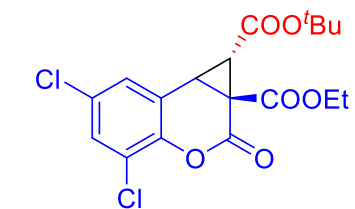

$11 \mathrm{i}$

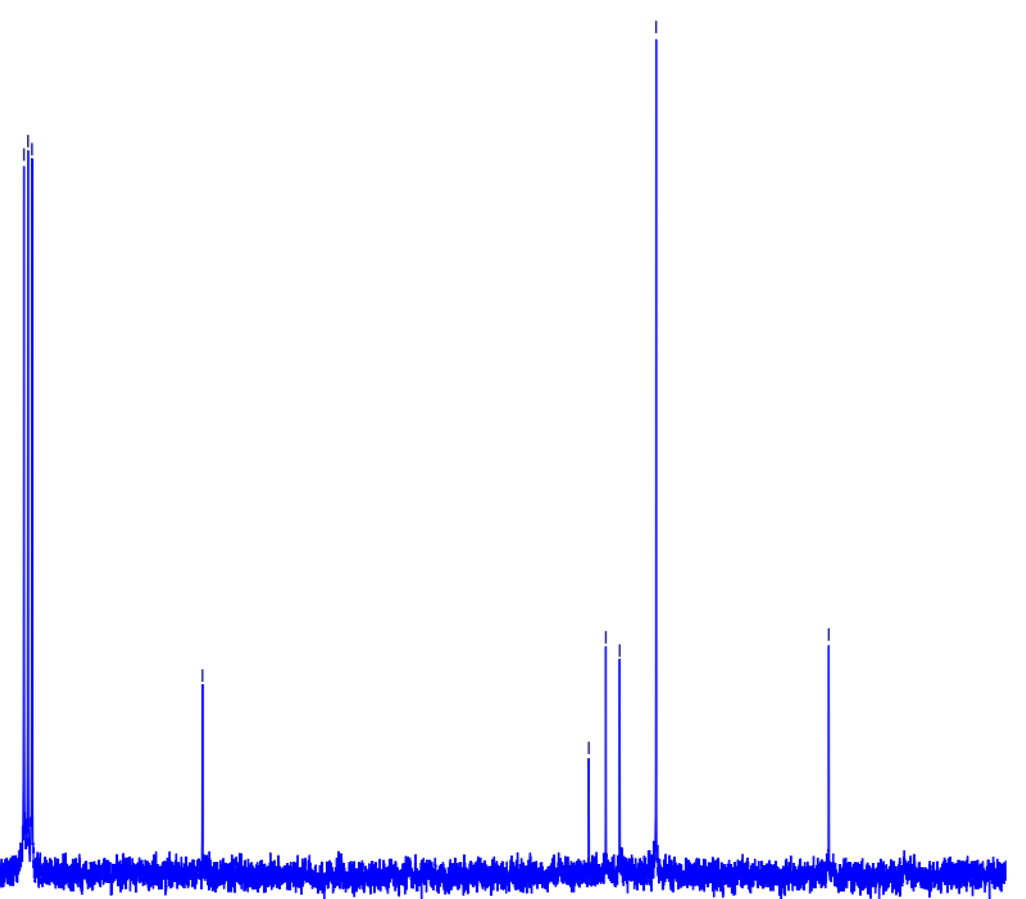

30

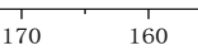

150

140

130

120

100

f1 $\stackrel{90}{(\mathrm{ppm})}$

80

70

30

20 
${ }^{1} \mathrm{H}$ NMR Spectrum (400 MHz, Chloroform- $d$ ) of $\mathbf{1 1} \mathbf{j}$

$$
\text { sjc-h-29-2 }
$$

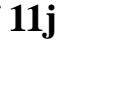

.

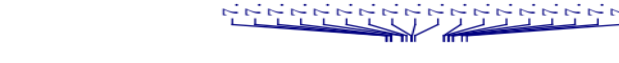
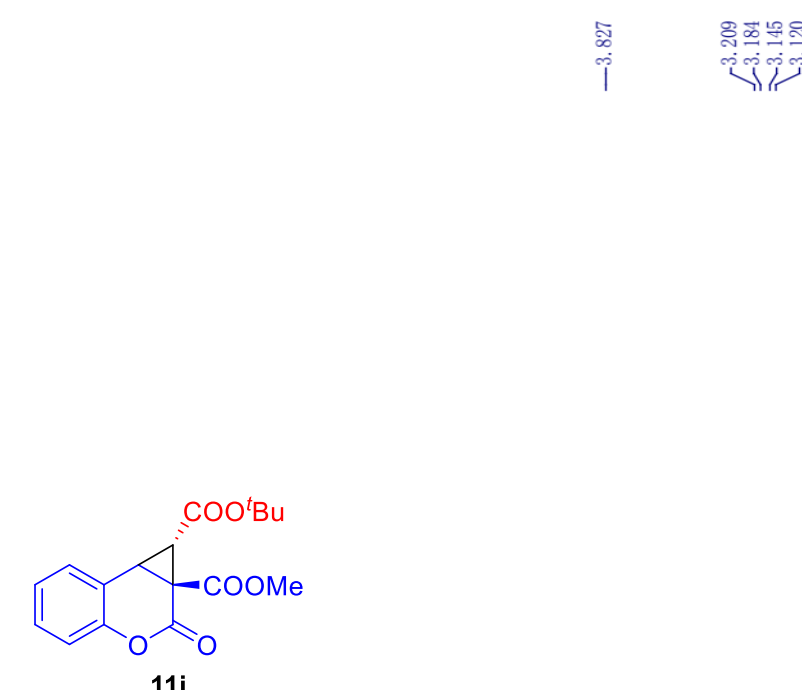

$11 \mathrm{j}$

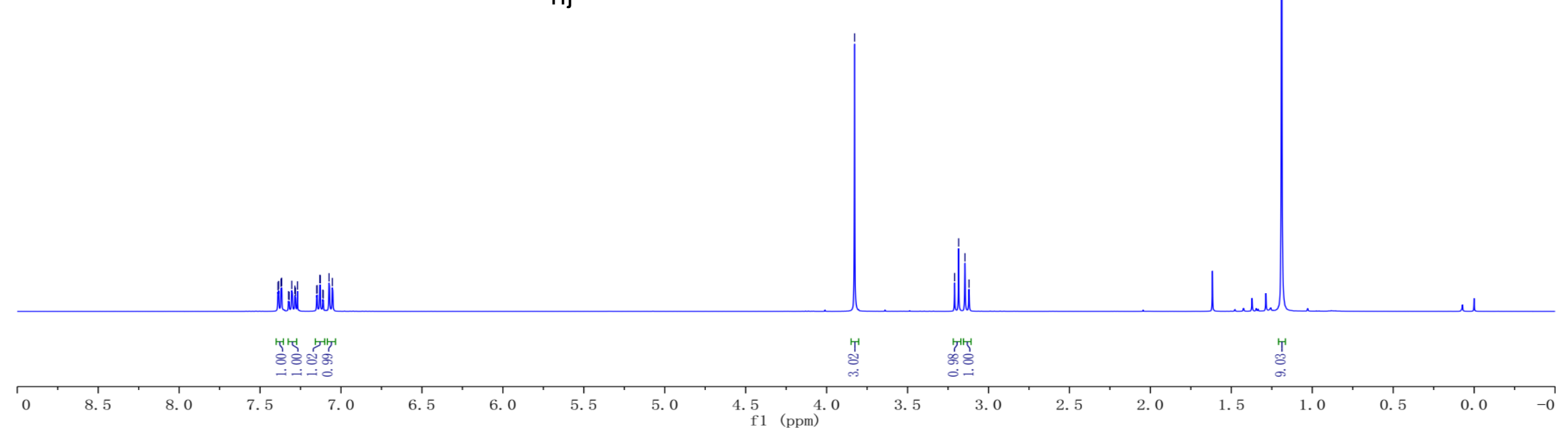


${ }^{13} \mathrm{C}\left\{{ }^{1} \mathrm{H}\right\}$ NMR Spectrum (101 MHz, Chloroform- $d$ ) of $\mathbf{1 1} \mathbf{j}$
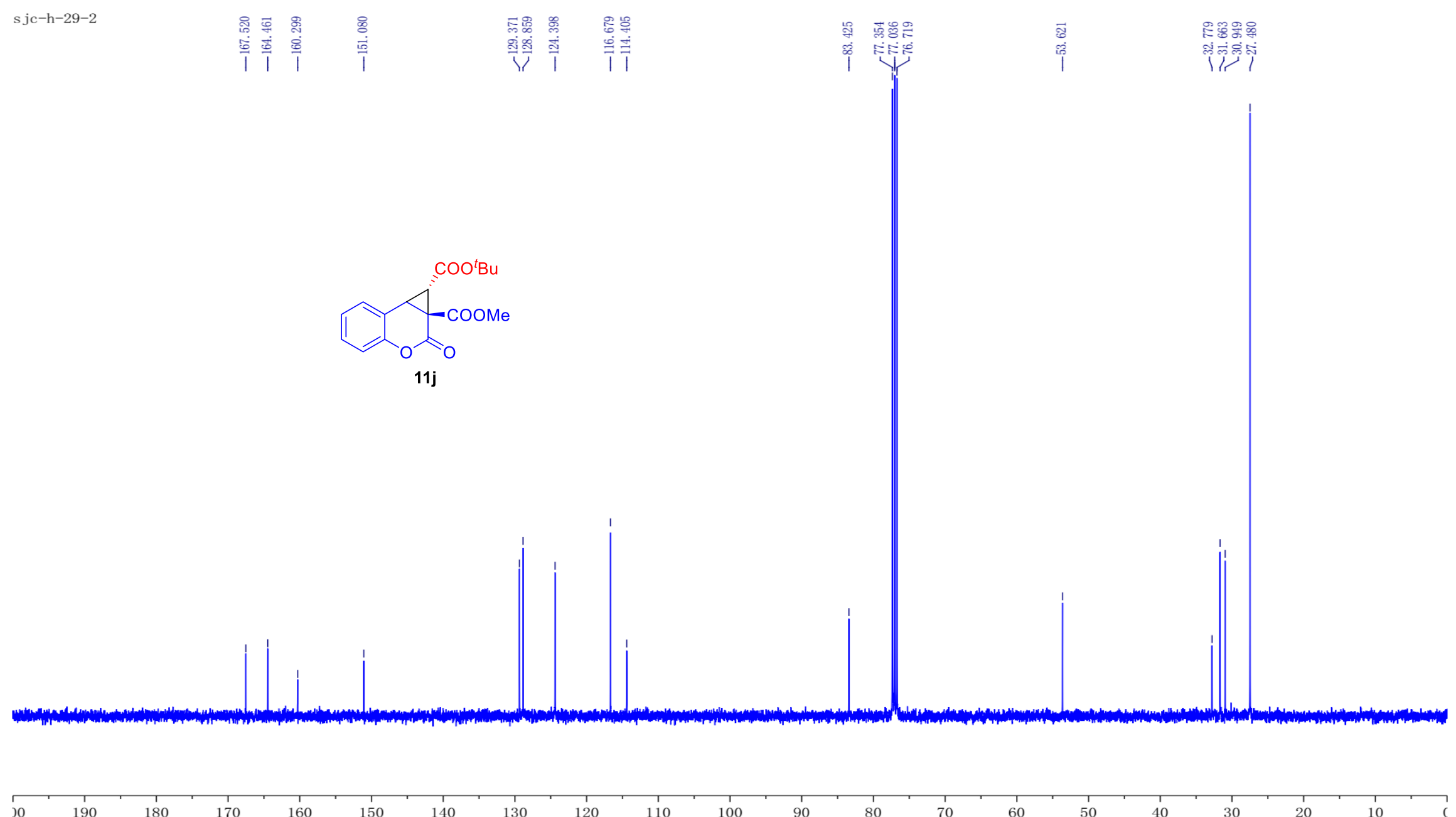

$40 \quad 130$

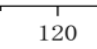

$10 \quad 100$ 
${ }^{1} \mathrm{H}$ NMR Spectrum (400 MHz, Chloroform- $d$ ) of $\mathbf{1 1 k}$

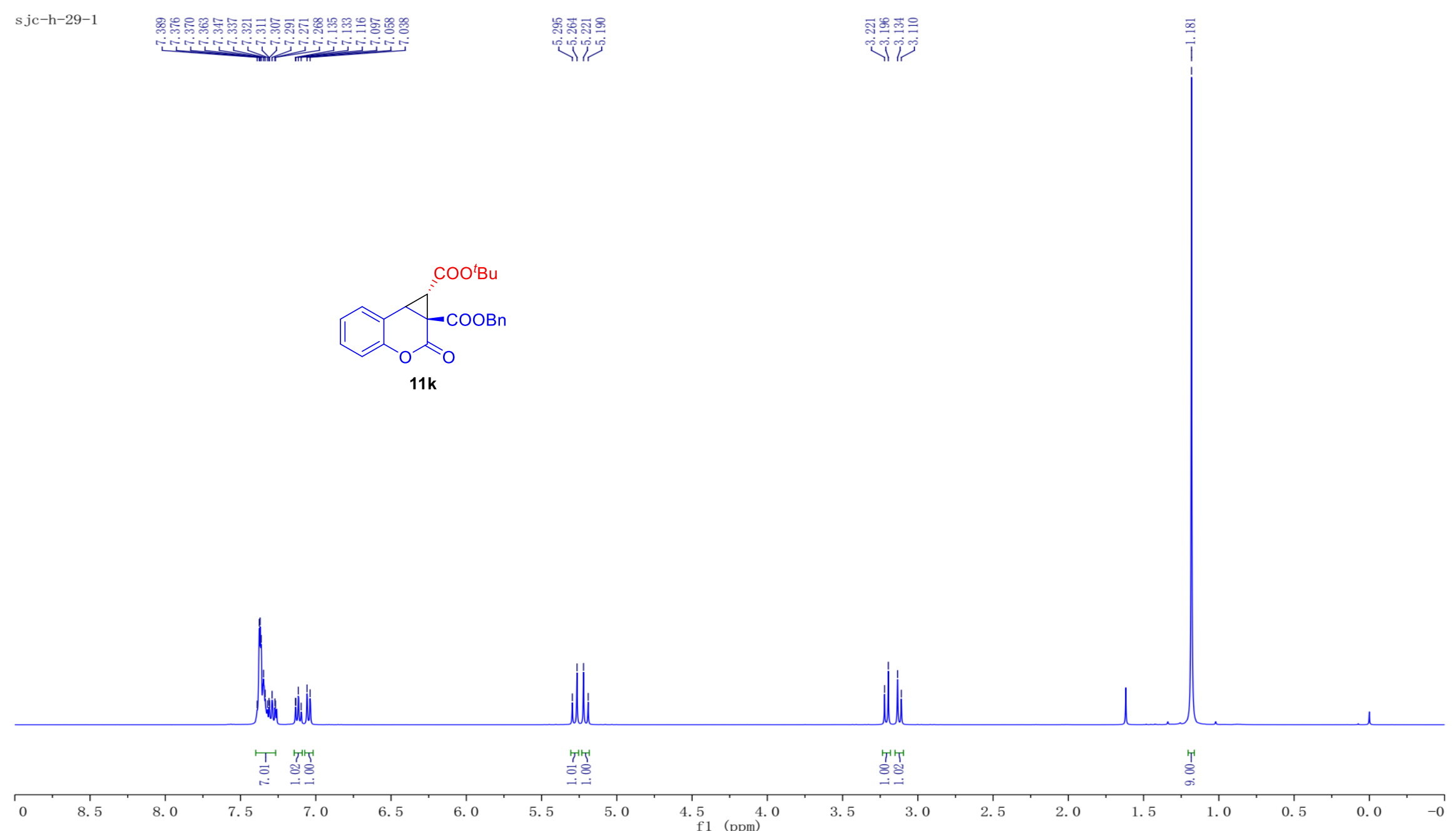


${ }^{13} \mathrm{C}\left\{{ }^{1} \mathrm{H}\right\}$ NMR Spectrum (101 MHz, Chloroform- $d$ ) of 11k

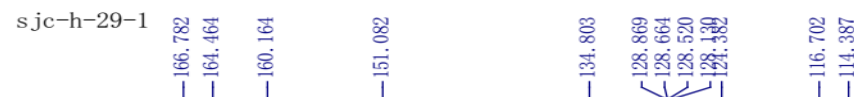

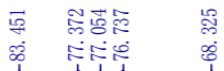

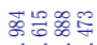

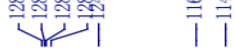

1

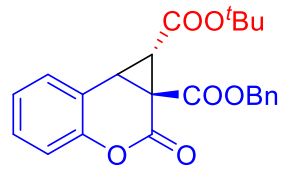

$11 \mathrm{k}$
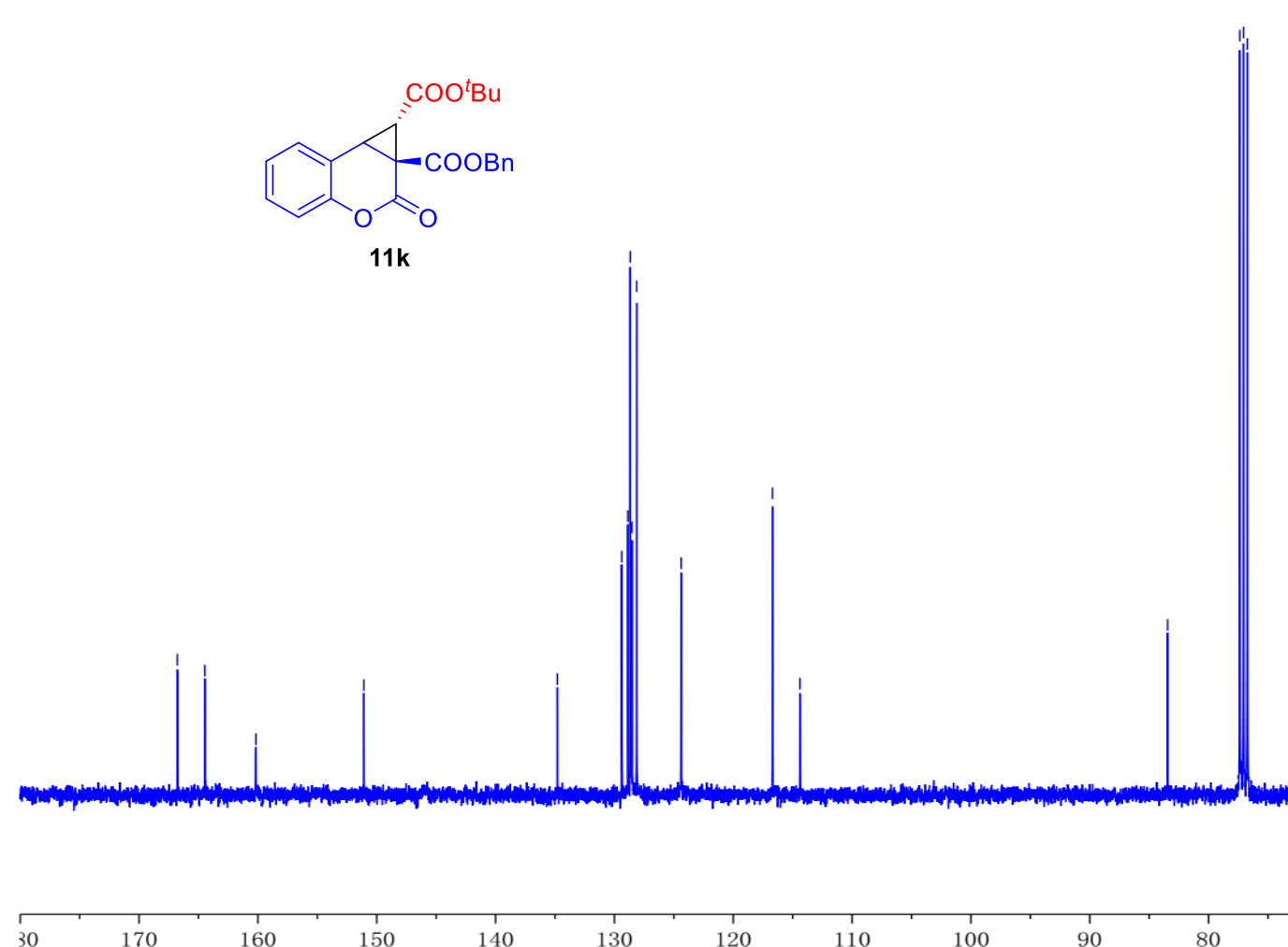

150

140

130

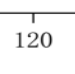

110

100

f1 $\stackrel{90}{(\mathrm{ppm})}$

80

70

60

50

30

20

10 
${ }^{1} \mathrm{H}$ NMR Spectrum (400 MHz, Chloroform- $d$ ) of $\mathbf{1 5 a}$

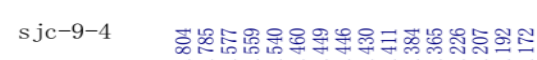

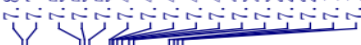

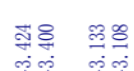

iं i

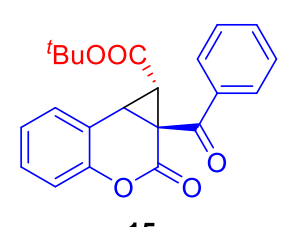

$15 a$

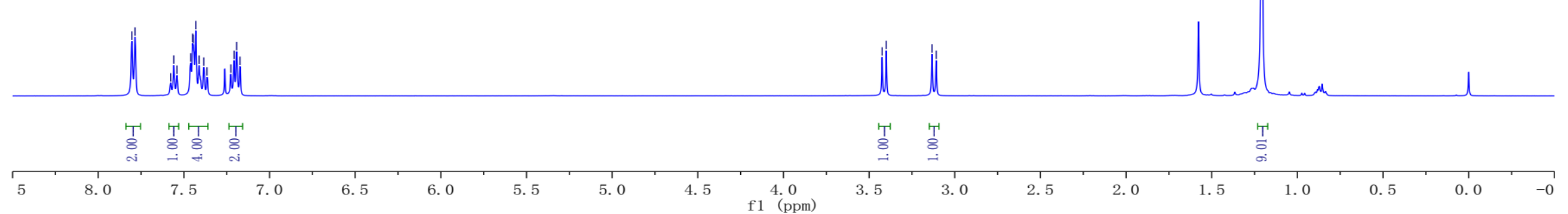


${ }^{13} \mathrm{C}\left\{{ }^{1} \mathrm{H}\right\}$ NMR Spectrum (101 MHz, Chloroform- $d$ ) of $\mathbf{1 5 a}$

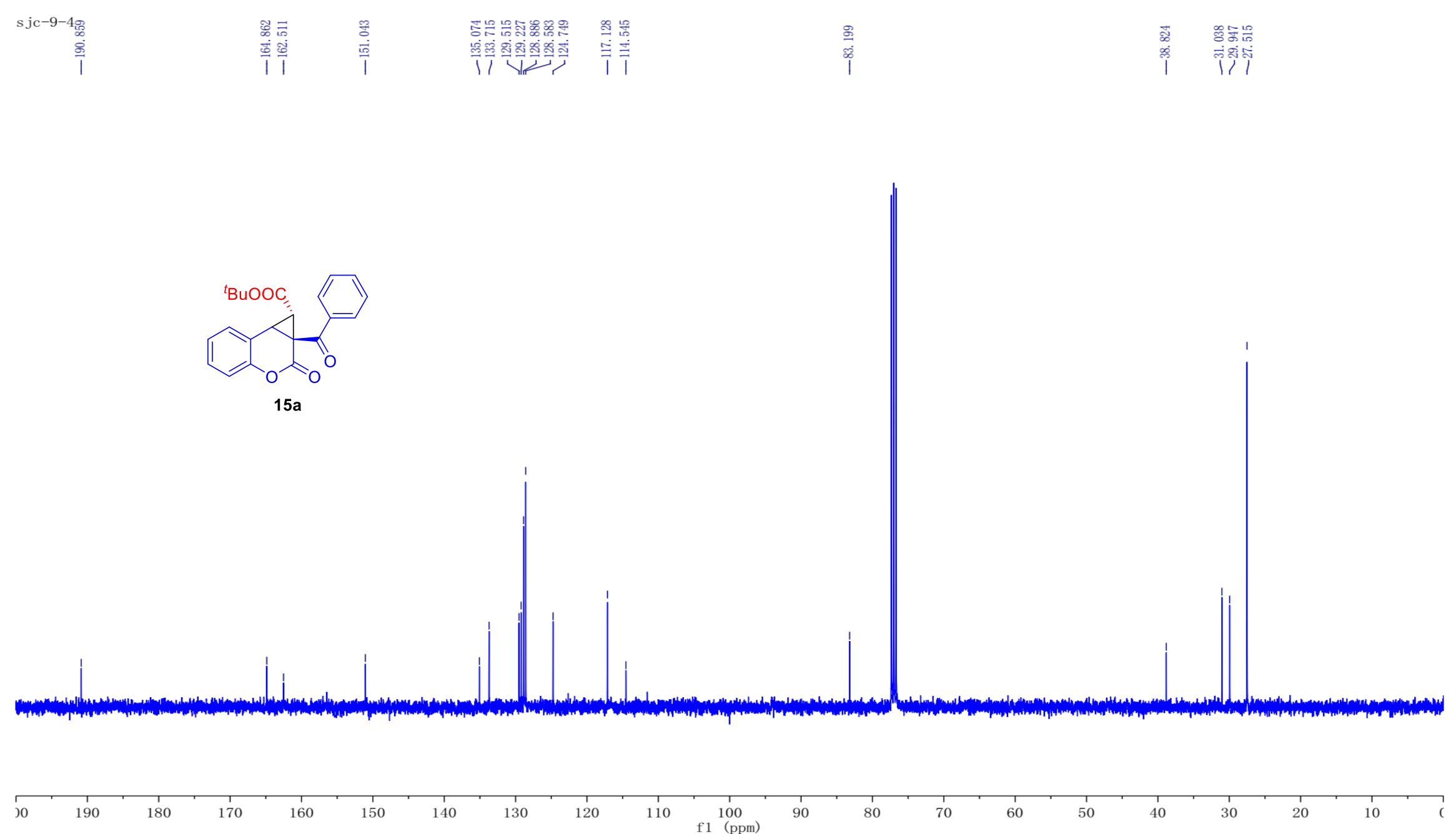


${ }^{1} \mathrm{H}$ NMR Spectrum (400 MHz, Chloroform- $d$ ) of $\mathbf{1 5 b}$

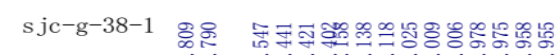

i ivivinivisis

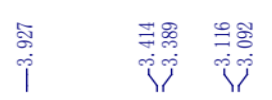

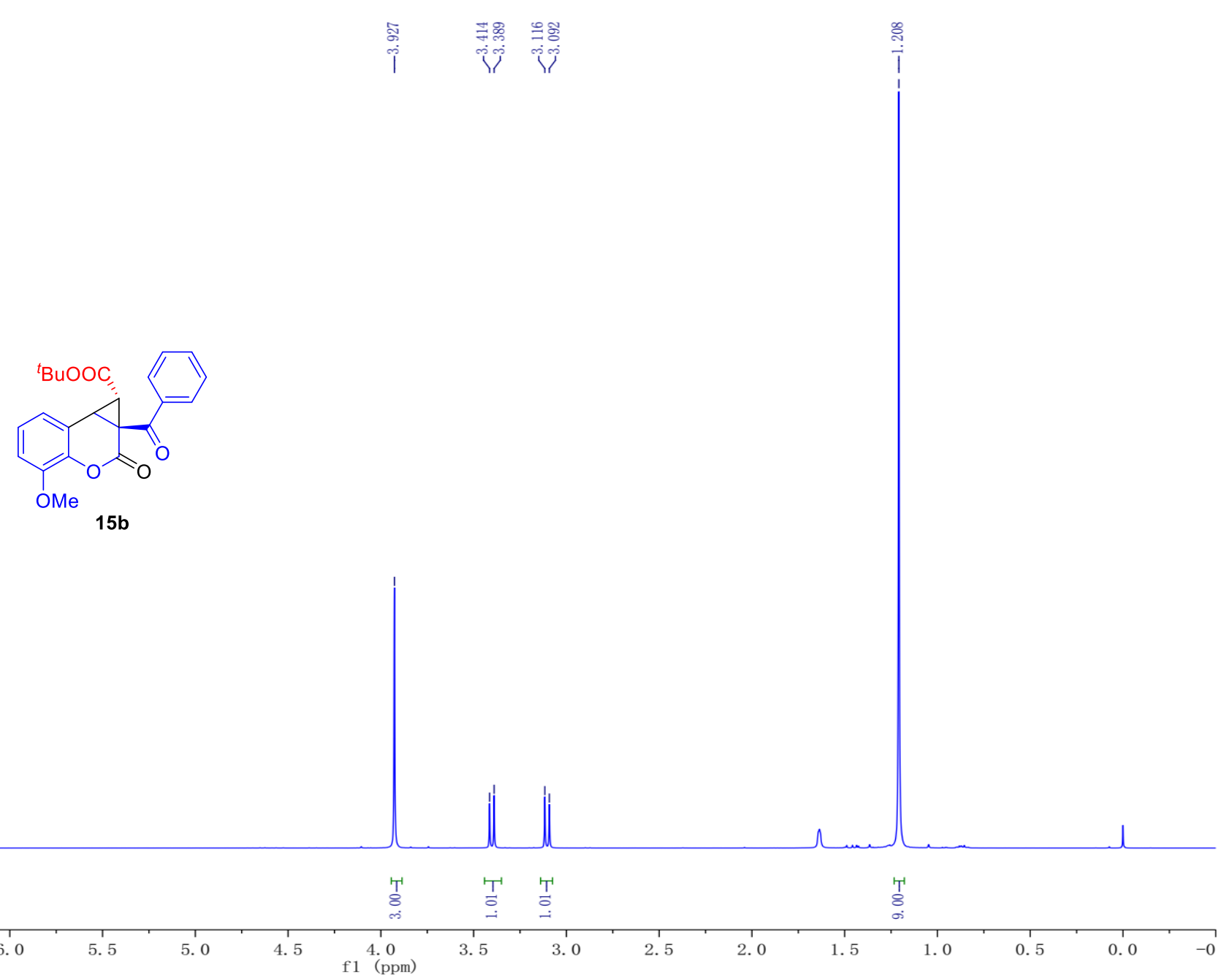

S30 
${ }^{13} \mathrm{C}\left\{{ }^{1} \mathrm{H}\right\}$ NMR Spectrum (101 MHz, Chloroform- $d$ ) of $\mathbf{1 5 b}$
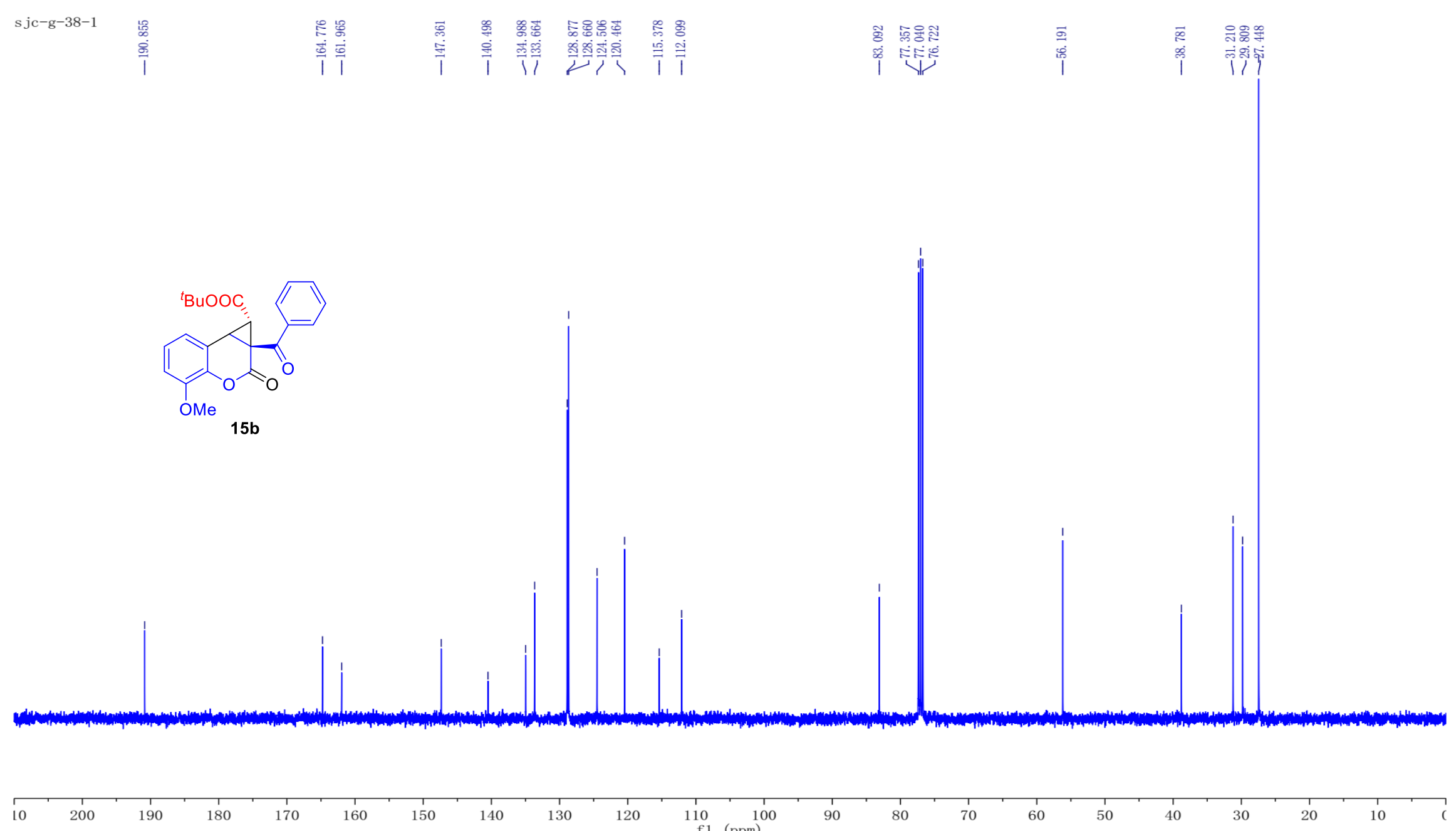
${ }^{1} \mathrm{H}$ NMR Spectrum (400 MHz, Chloroform- $d$ ) of $\mathbf{1 5 c}$

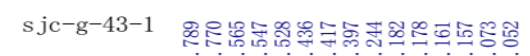

ivivinitivisis
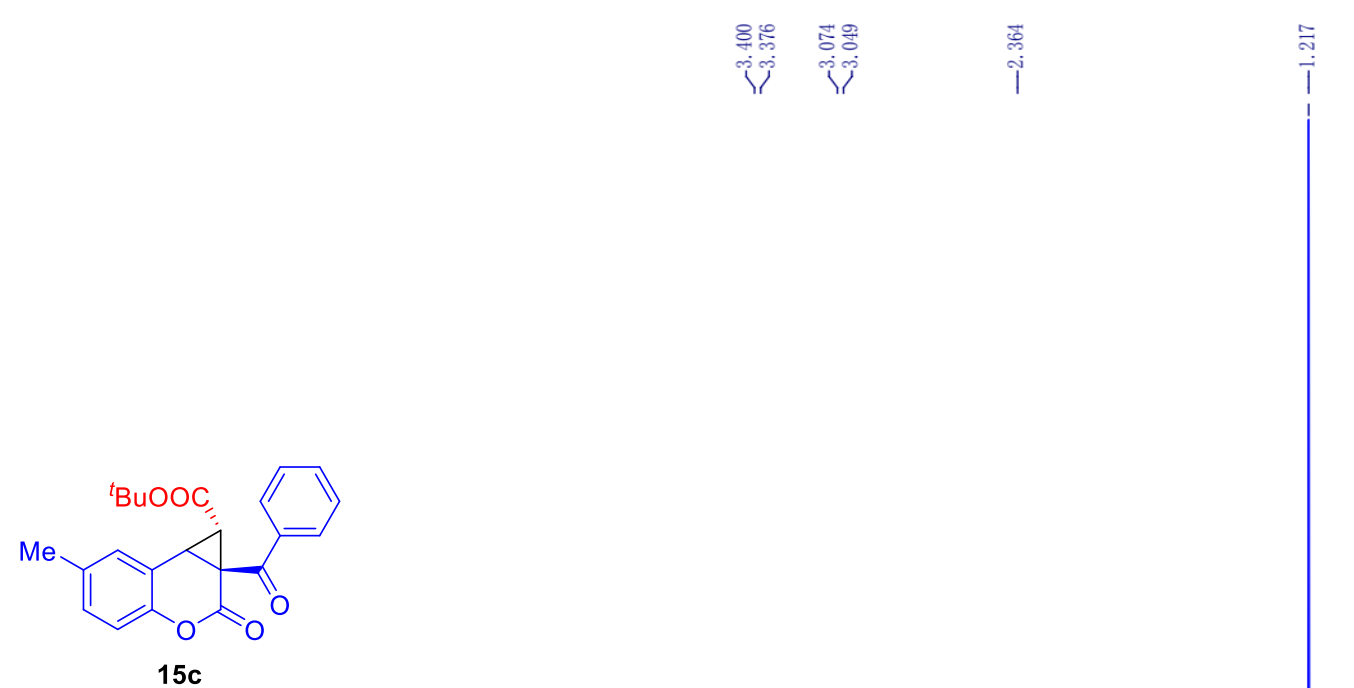

$15 \mathrm{c}$

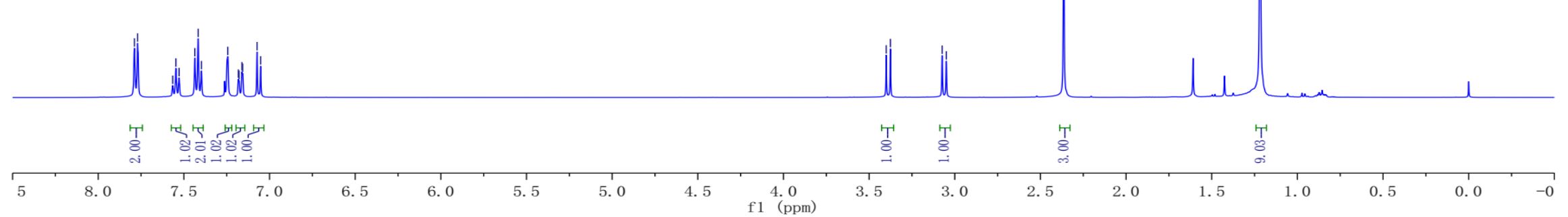


${ }^{13} \mathrm{C}\left\{{ }^{1} \mathrm{H}\right\}$ NMR Spectrum (101 MHz, Chloroform- $d$ ) of $\mathbf{1 5 c}$
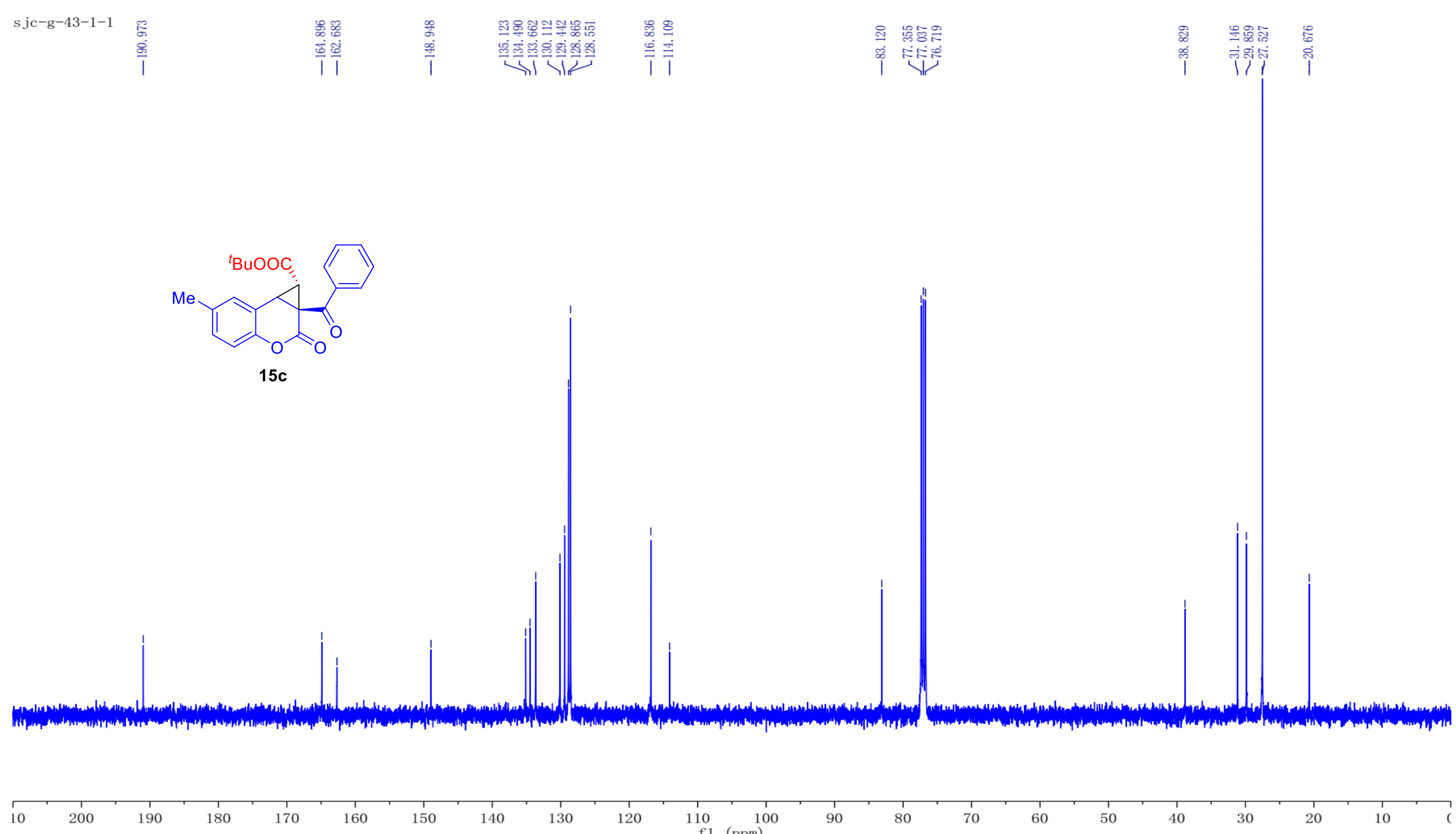
${ }^{1} \mathrm{H}$ NMR Spectrum (400 MHz, Chloroform- $d$ ) of $\mathbf{1 5 d}$

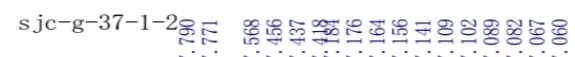

iv ivivinimin

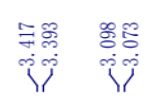

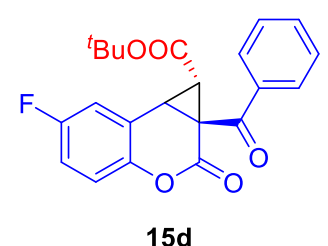

$15 d$

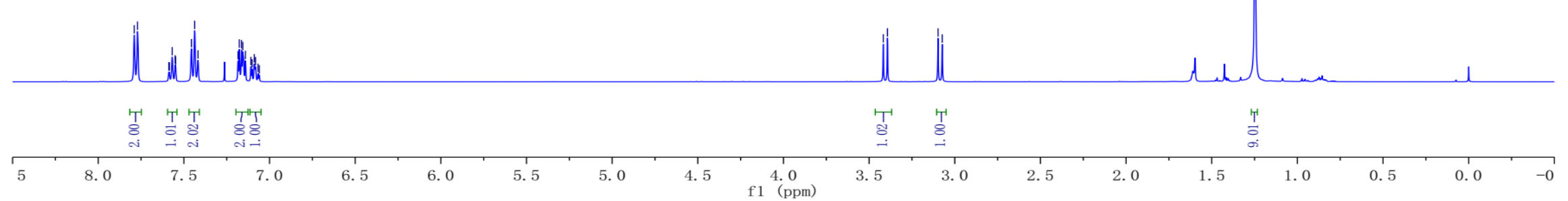


${ }^{13} \mathrm{C}\left\{{ }^{1} \mathrm{H}\right\}$ NMR Spectrum (101 MHz, Chloroform- $d$ ) of 15d
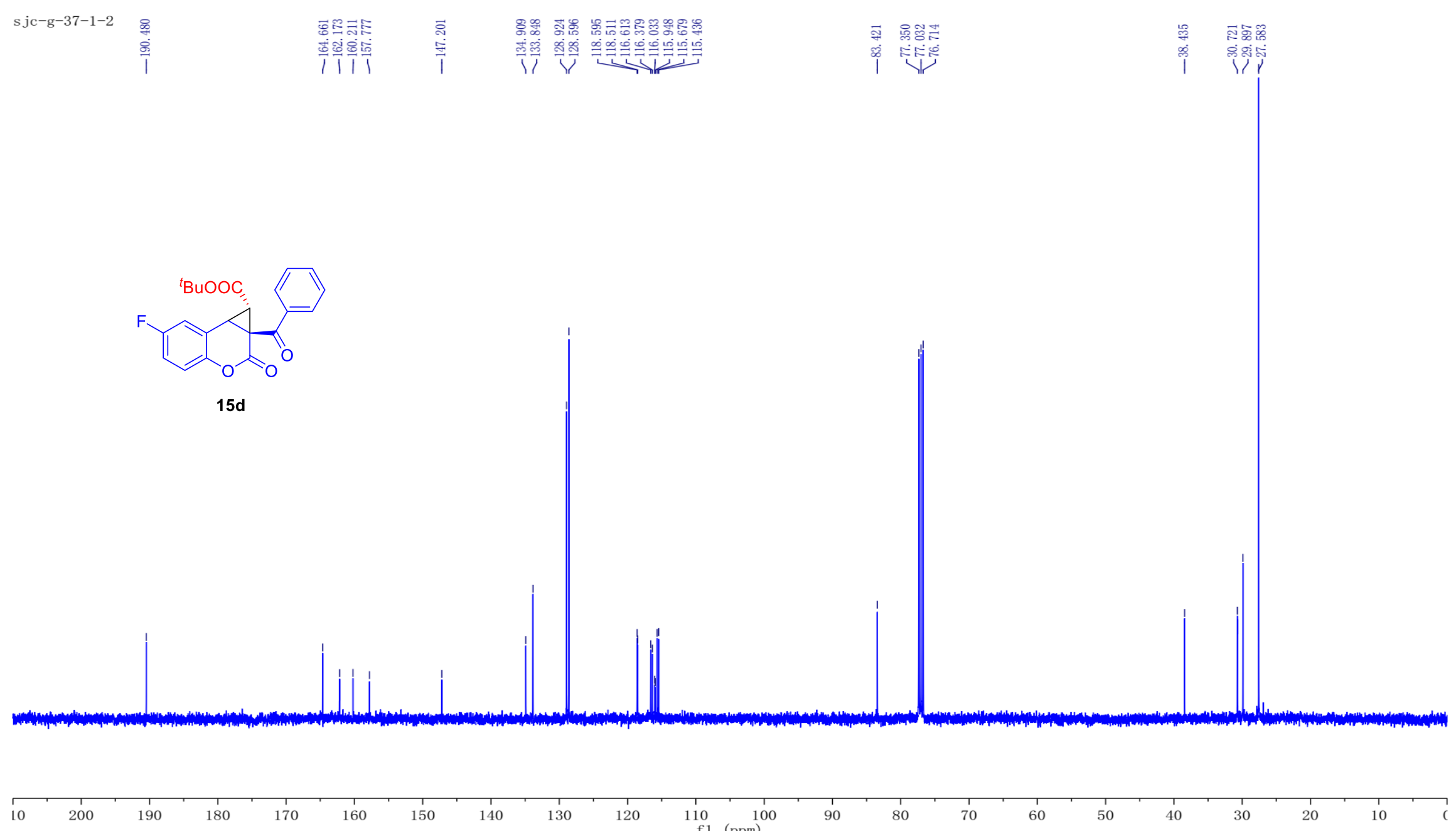
${ }^{19}$ F NMR (282 MHz, Chloroform- $d$ ) of $\mathbf{1 5 d}$

sjc-g-37-1-2

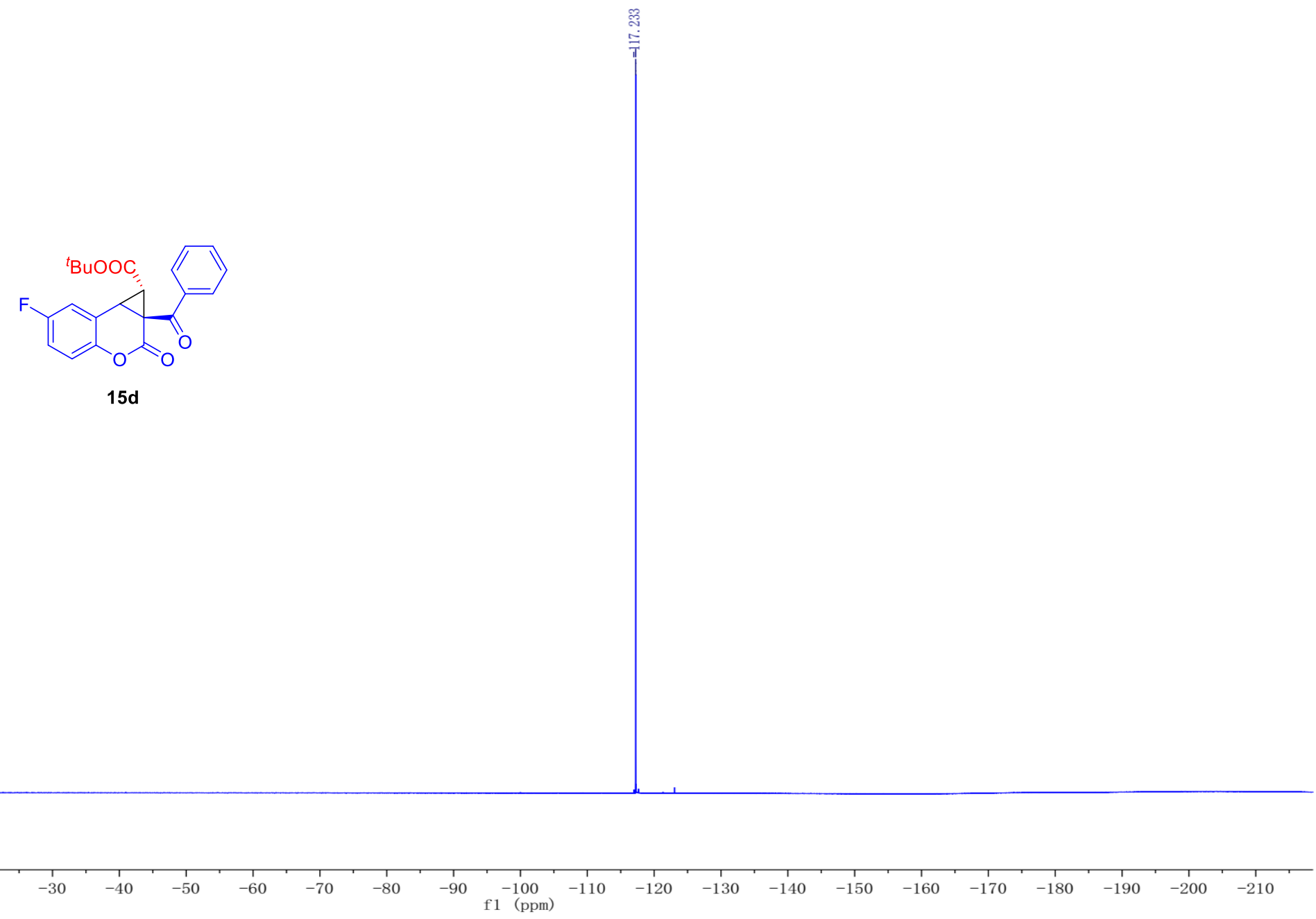


${ }^{1} \mathrm{H}$ NMR Spectrum (400 MHz, Chloroform- $d$ ) of $\mathbf{1 5 e}$

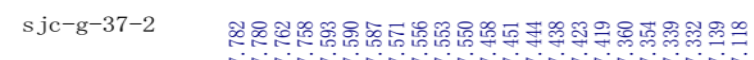

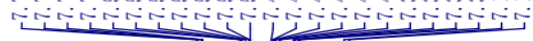

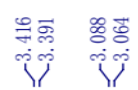

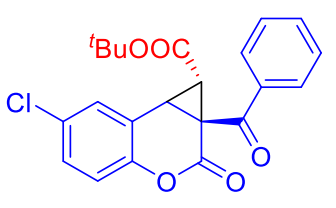

$15 e$

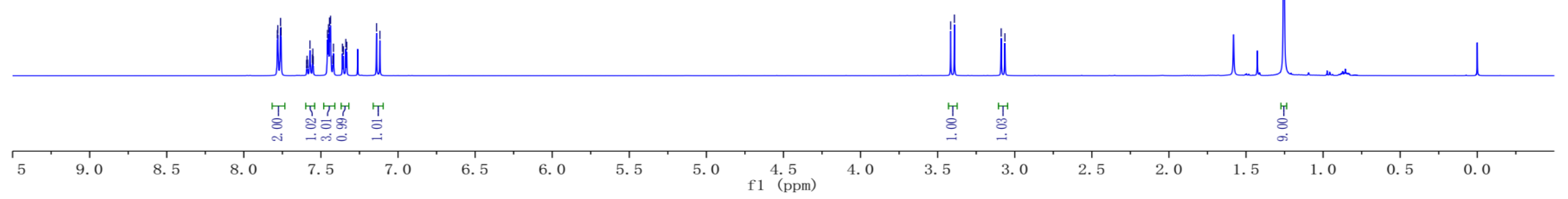


${ }^{13} \mathrm{C}\left\{{ }^{1} \mathrm{H}\right\}$ NMR Spectrum (101 MHz, Chloroform- $d$ ) of 15e

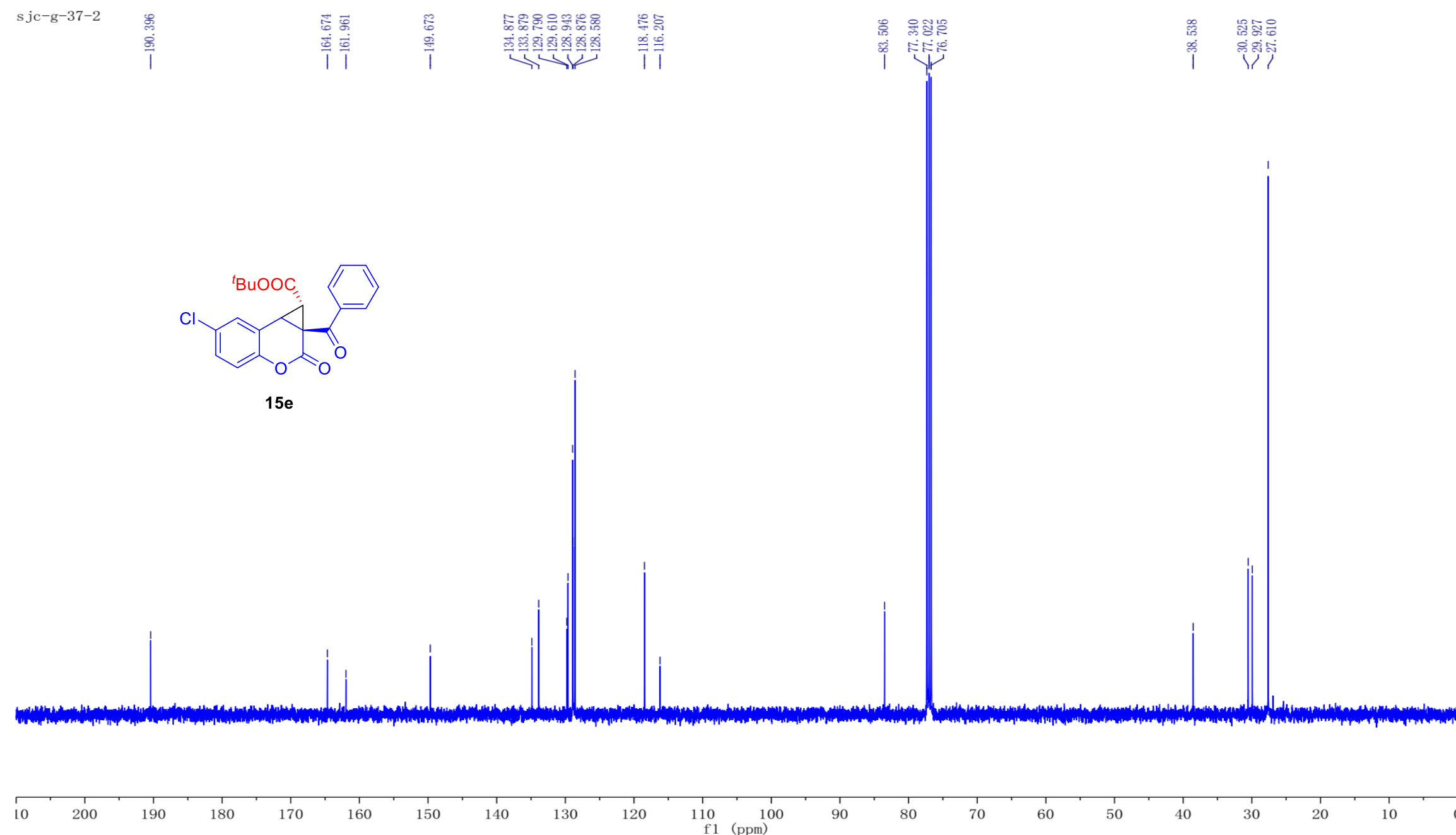


${ }^{1} \mathrm{H}$ NMR Spectrum (400 MHz, Chloroform- $d$ ) of $\mathbf{1 5 f}$
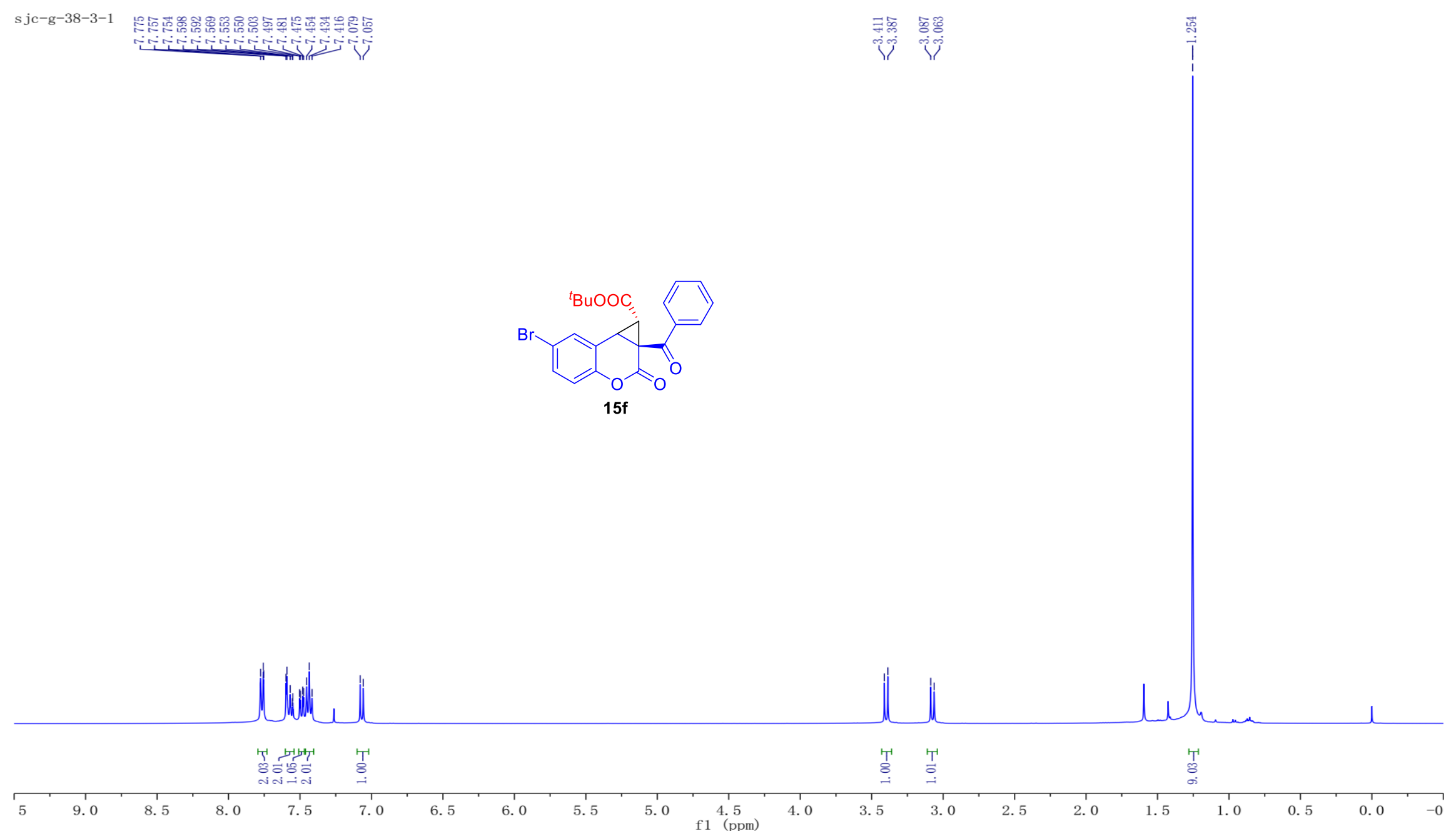
${ }^{13} \mathrm{C}\left\{{ }^{1} \mathrm{H}\right\}$ NMR Spectrum (101 MHz, Chloroform- $d$ ) of $\mathbf{1 5 f}$
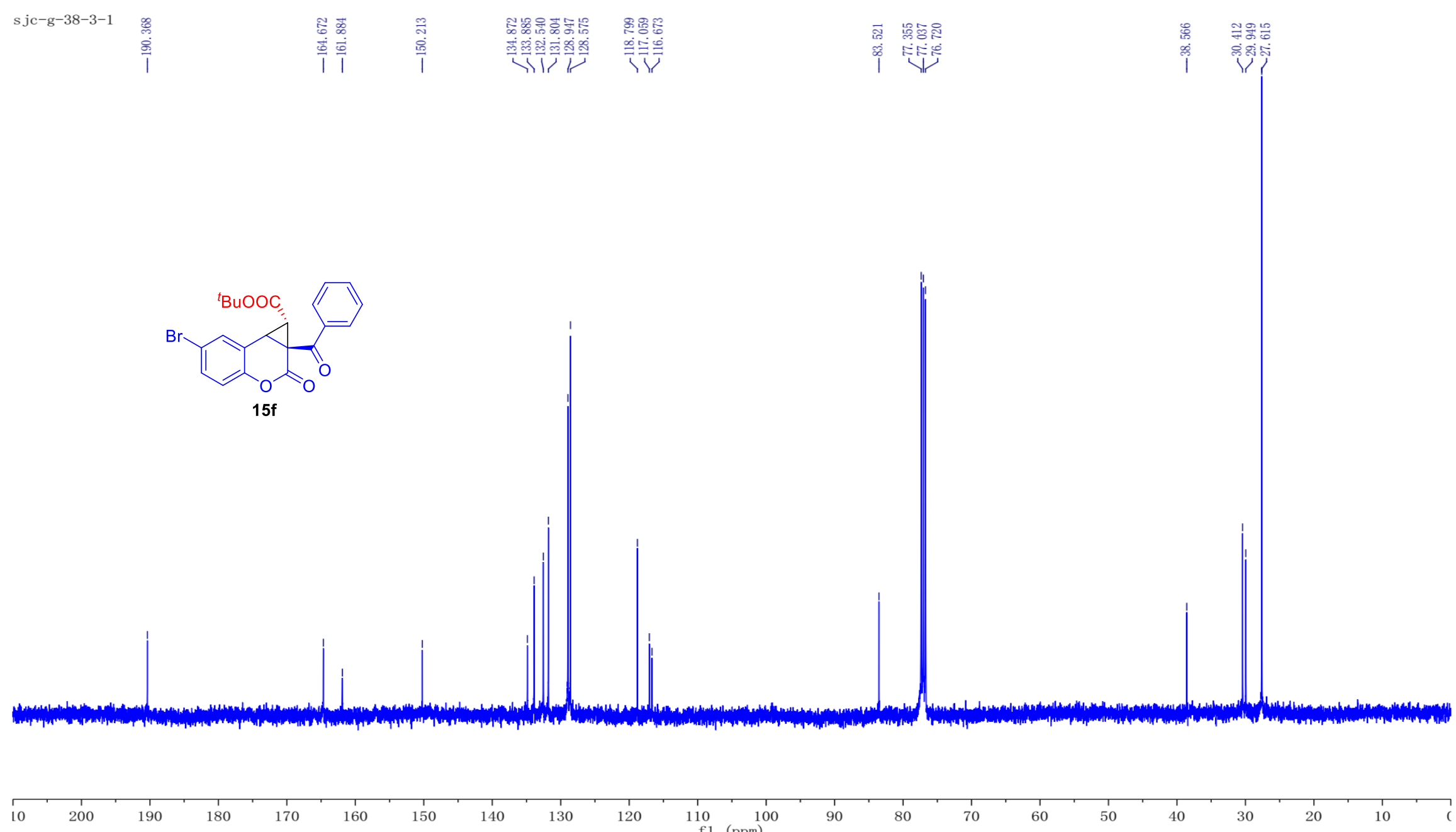
${ }^{1} \mathrm{H}$ NMR Spectrum (400 MHz, Chloroform- $d$ ) of $\mathbf{1 5 g}$

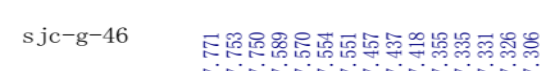

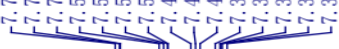

丞

ij

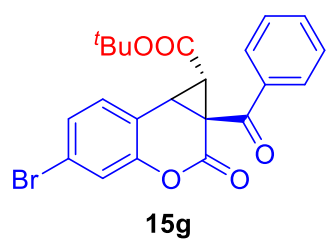

$15 \mathrm{~g}$

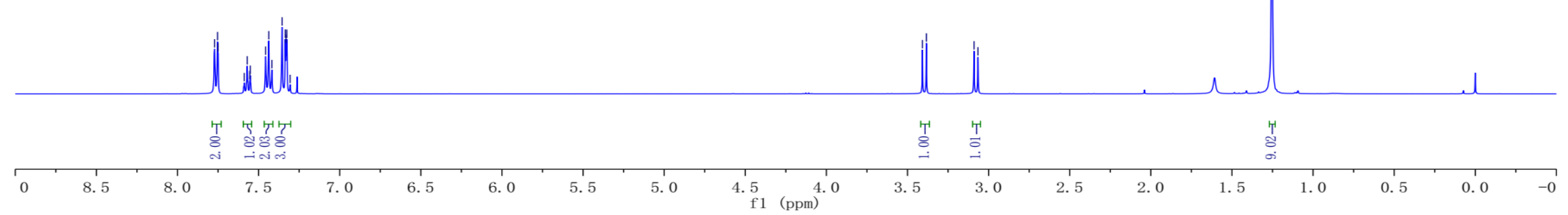

S41 
${ }^{13} \mathrm{C}\left\{{ }^{1} \mathrm{H}\right\}$ NMR Spectrum (101 MHz, Chloroform- $d$ ) of $\mathbf{1 5 g}$
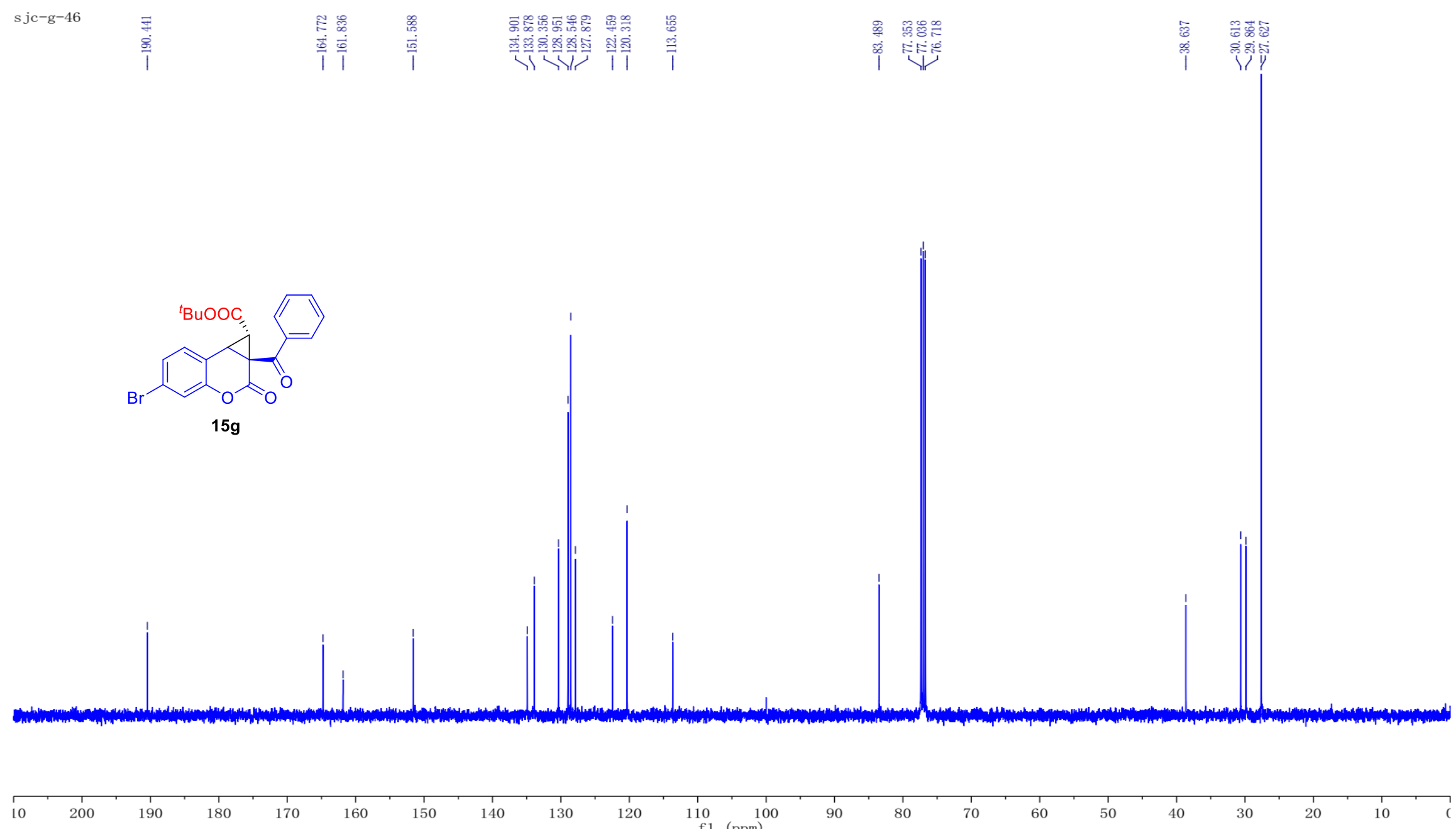
${ }^{1} \mathrm{H}$ NMR Spectrum (400 MHz, Chloroform- $d$ ) of $\mathbf{1 5 h}$

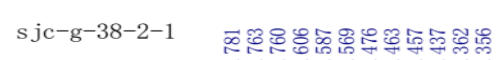

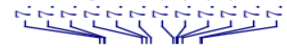

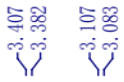

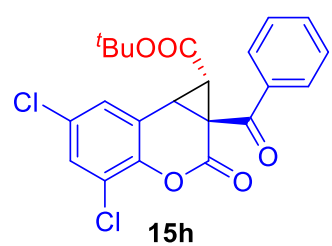

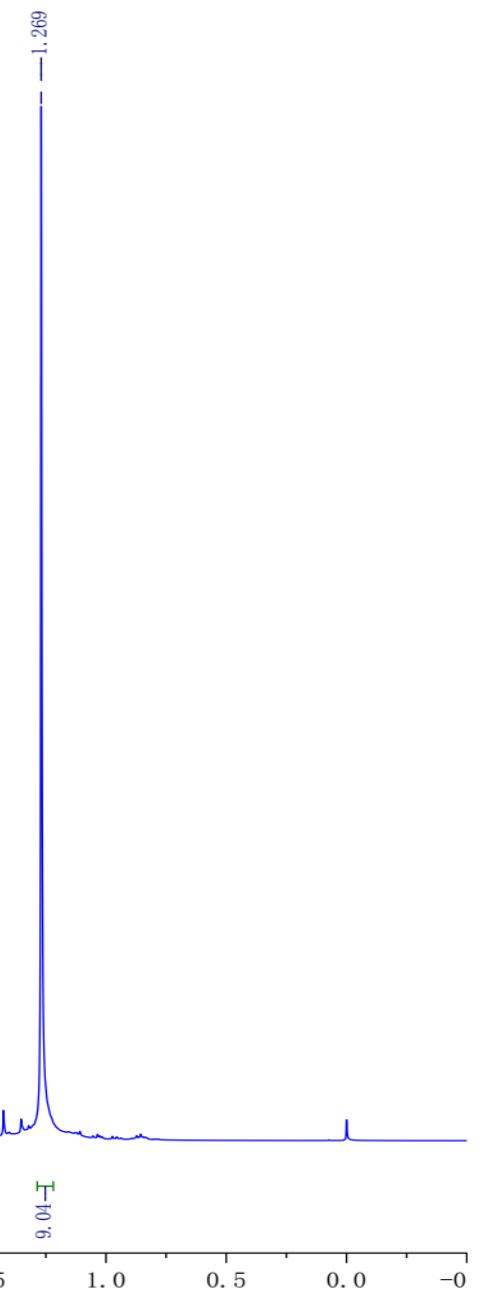


${ }^{13} \mathrm{C}\left\{{ }^{1} \mathrm{H}\right\}$ NMR Spectrum (101 MHz, Chloroform- $d$ ) of $\mathbf{1 5 h}$

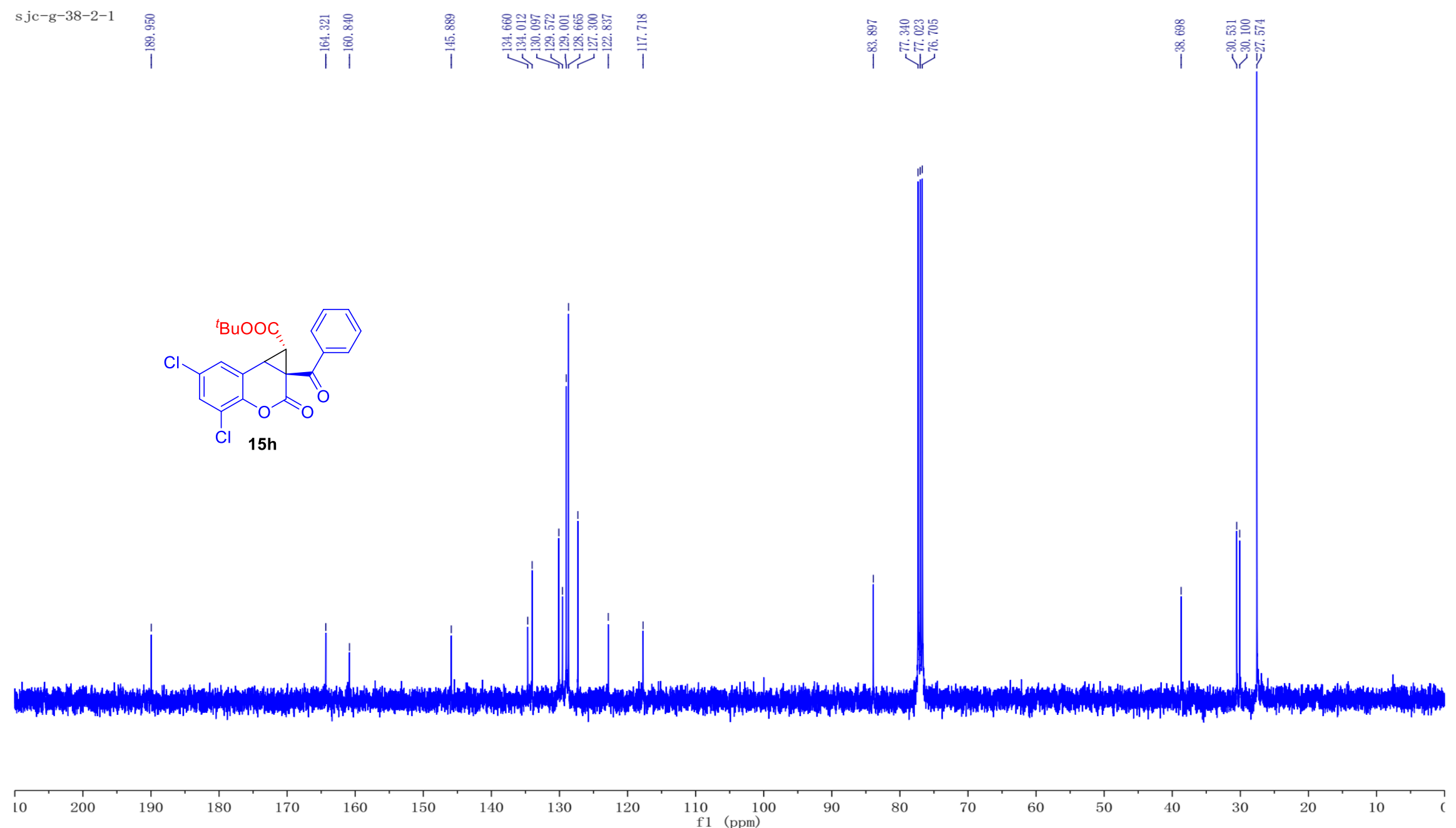


${ }^{1} \mathrm{H}$ NMR Spectrum (400 MHz, Chloroform- $d$ ) of $\mathbf{1 5 i}$

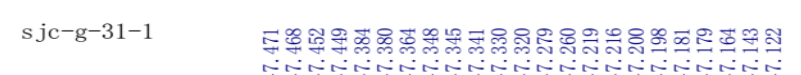

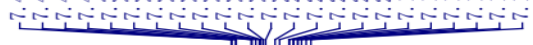

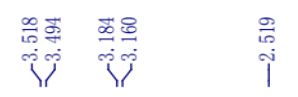

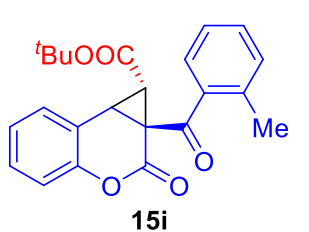

$15 i$

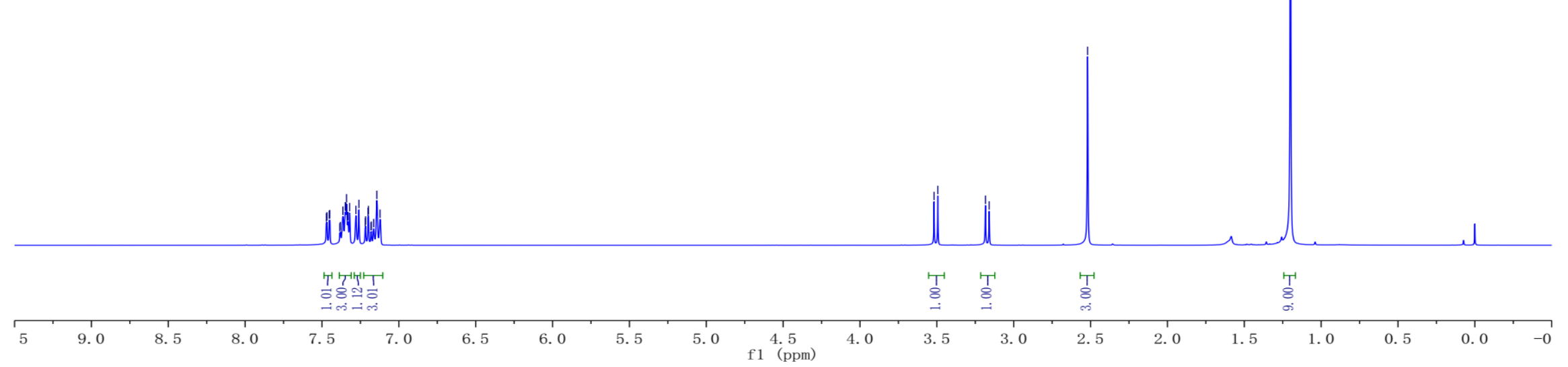

S45 
${ }^{13} \mathrm{C}\left\{{ }^{1} \mathrm{H}\right\}$ NMR Spectrum (101 MHz, Chloroform- $d$ ) of 15i
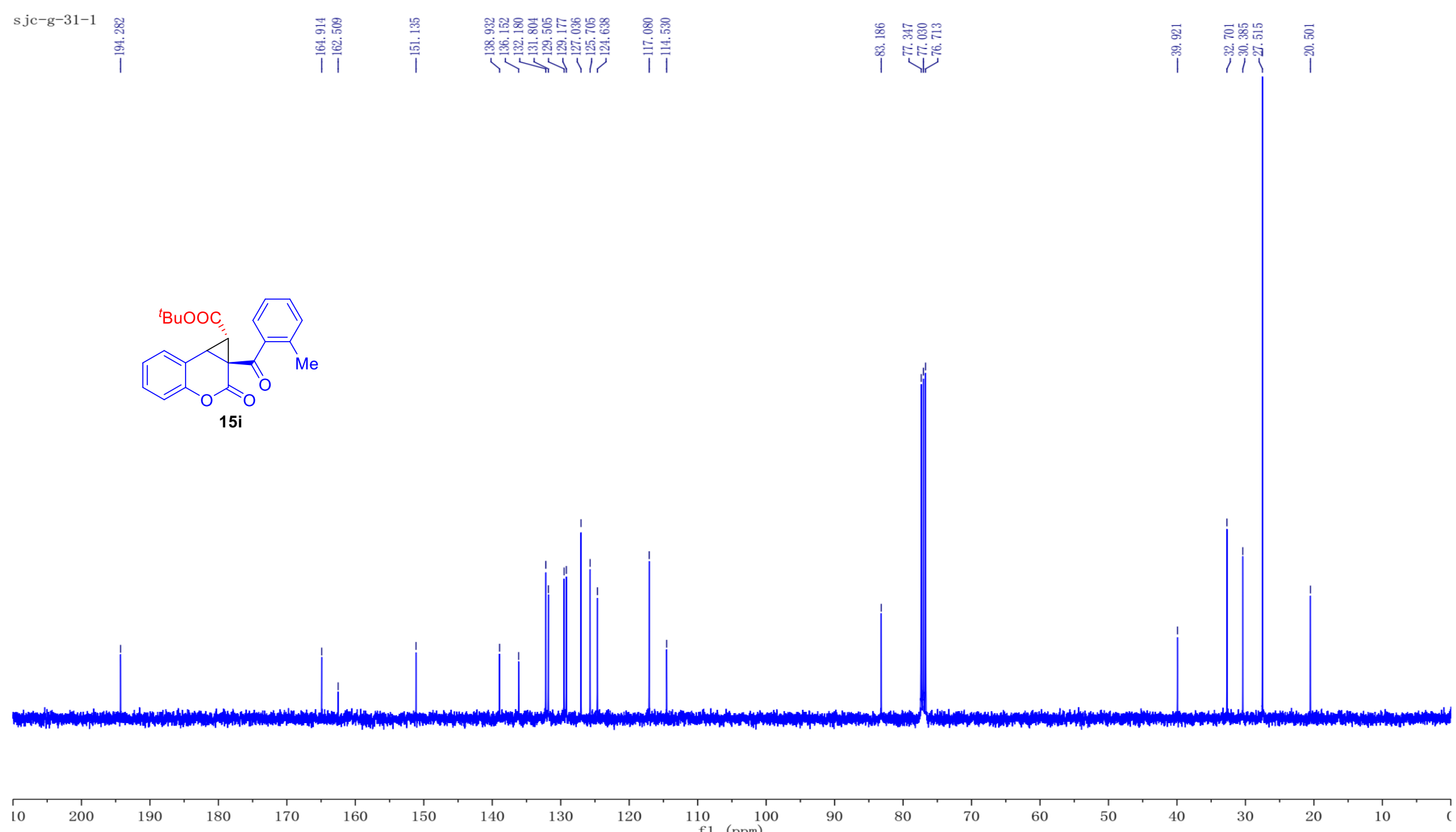
${ }^{1} \mathrm{H}$ NMR Spectrum (400 MHz, Chloroform- $d$ ) of $\mathbf{1 5 j}$

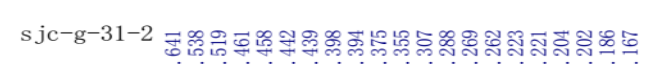

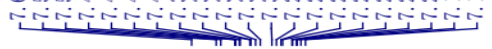

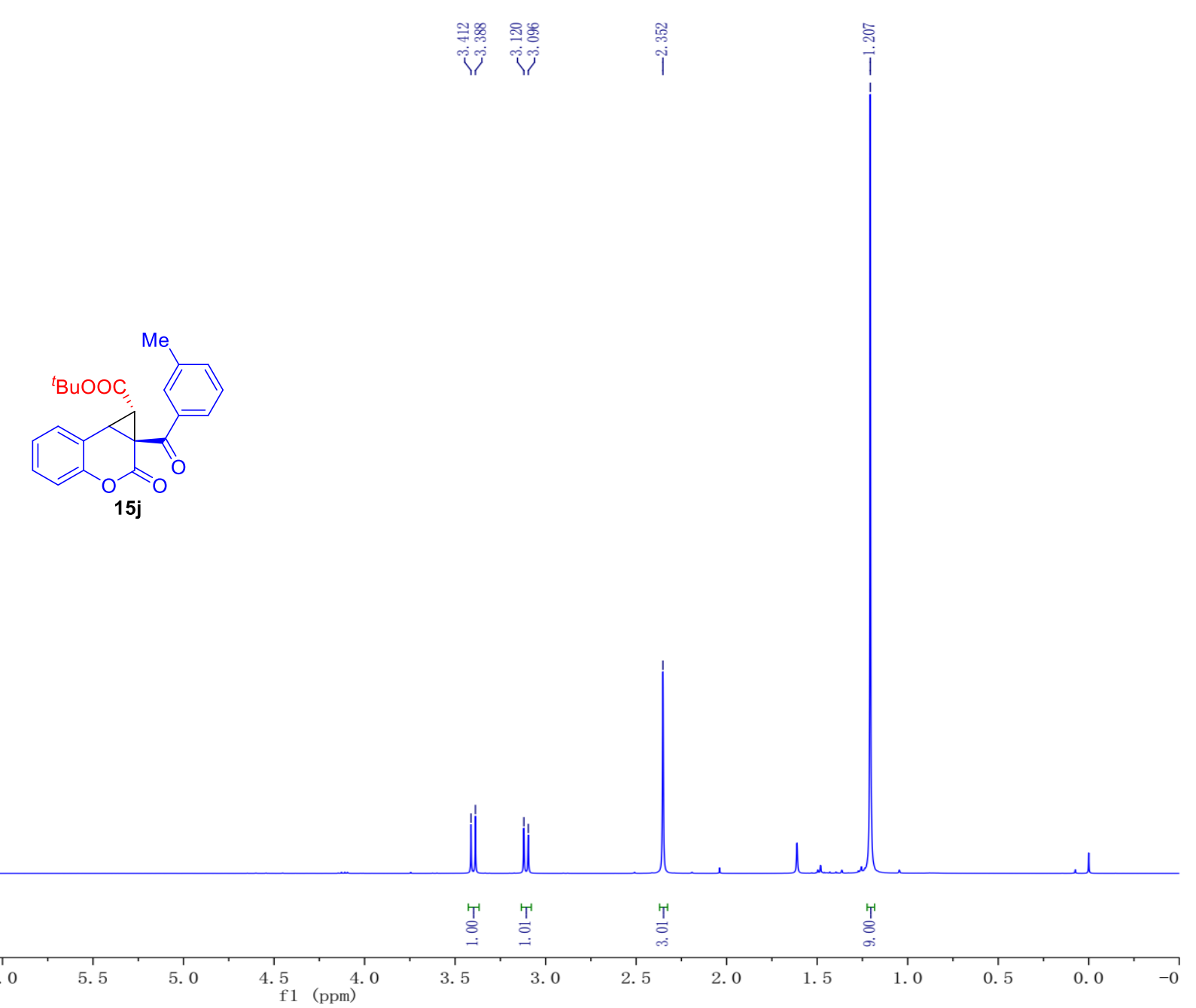

S47 
${ }^{13} \mathrm{C}\left\{{ }^{1} \mathrm{H}\right\}$ NMR Spectrum (101 MHz, Chloroform- $d$ ) of $\mathbf{1 5 j}$
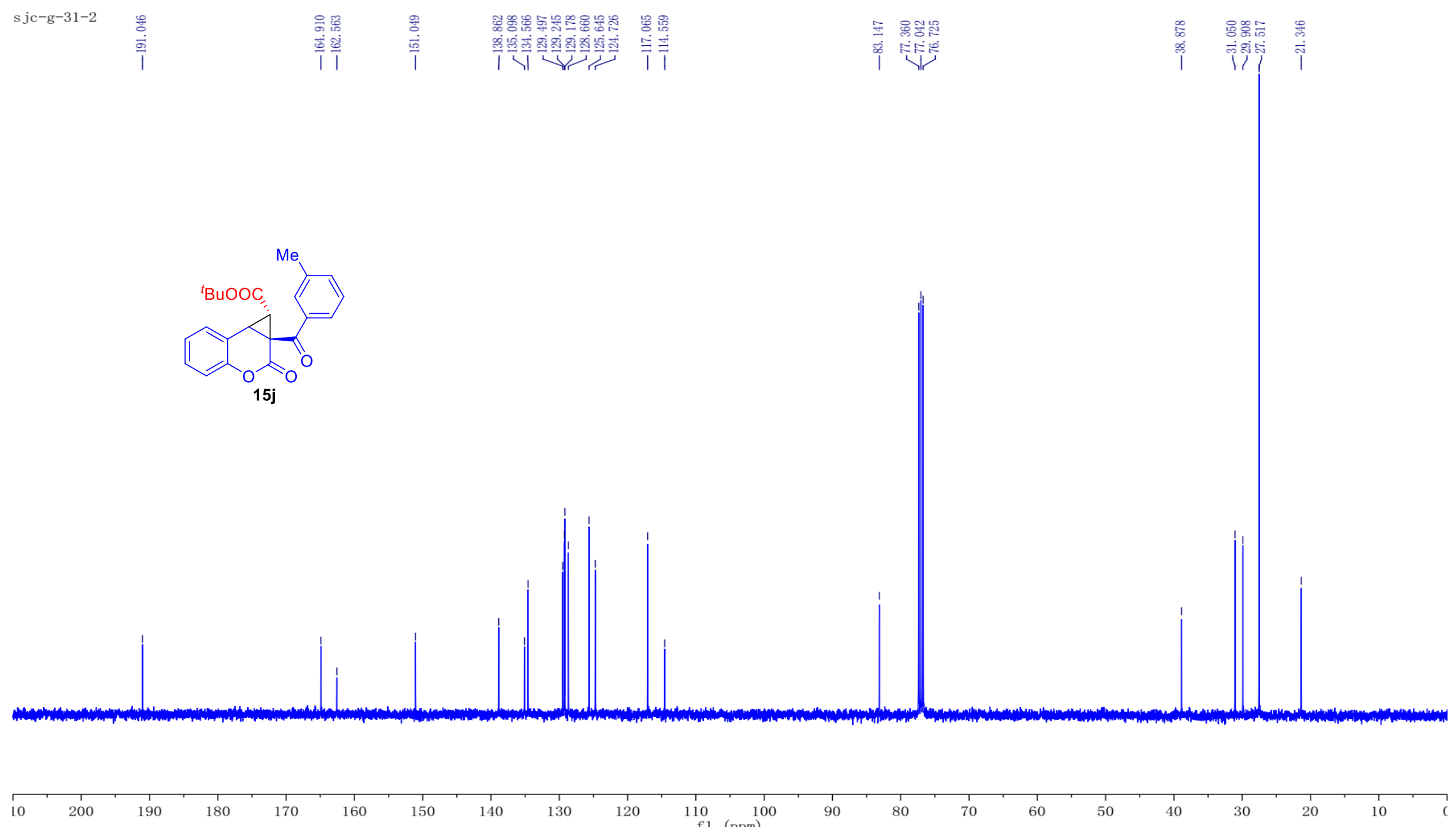
${ }^{1} \mathrm{H}$ NMR Spectrum (400 MHz, Chloroform- $d$ ) of $\mathbf{1 5 k}$

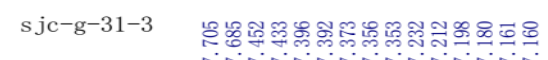

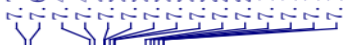

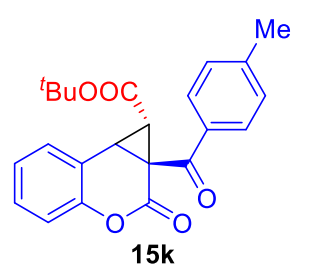

$15 \mathrm{k}$

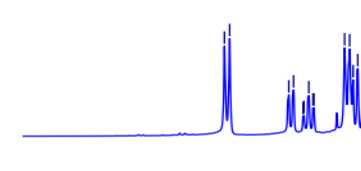

int

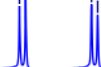

when

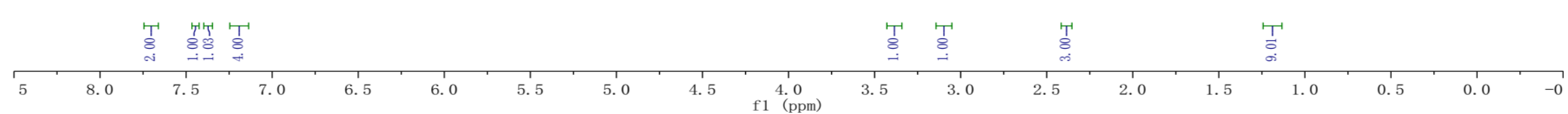


${ }^{13} \mathrm{C}\left\{{ }^{1} \mathrm{H}\right\}$ NMR Spectrum (101 MHz, Chloroform- $d$ ) of $\mathbf{1 5 k}$
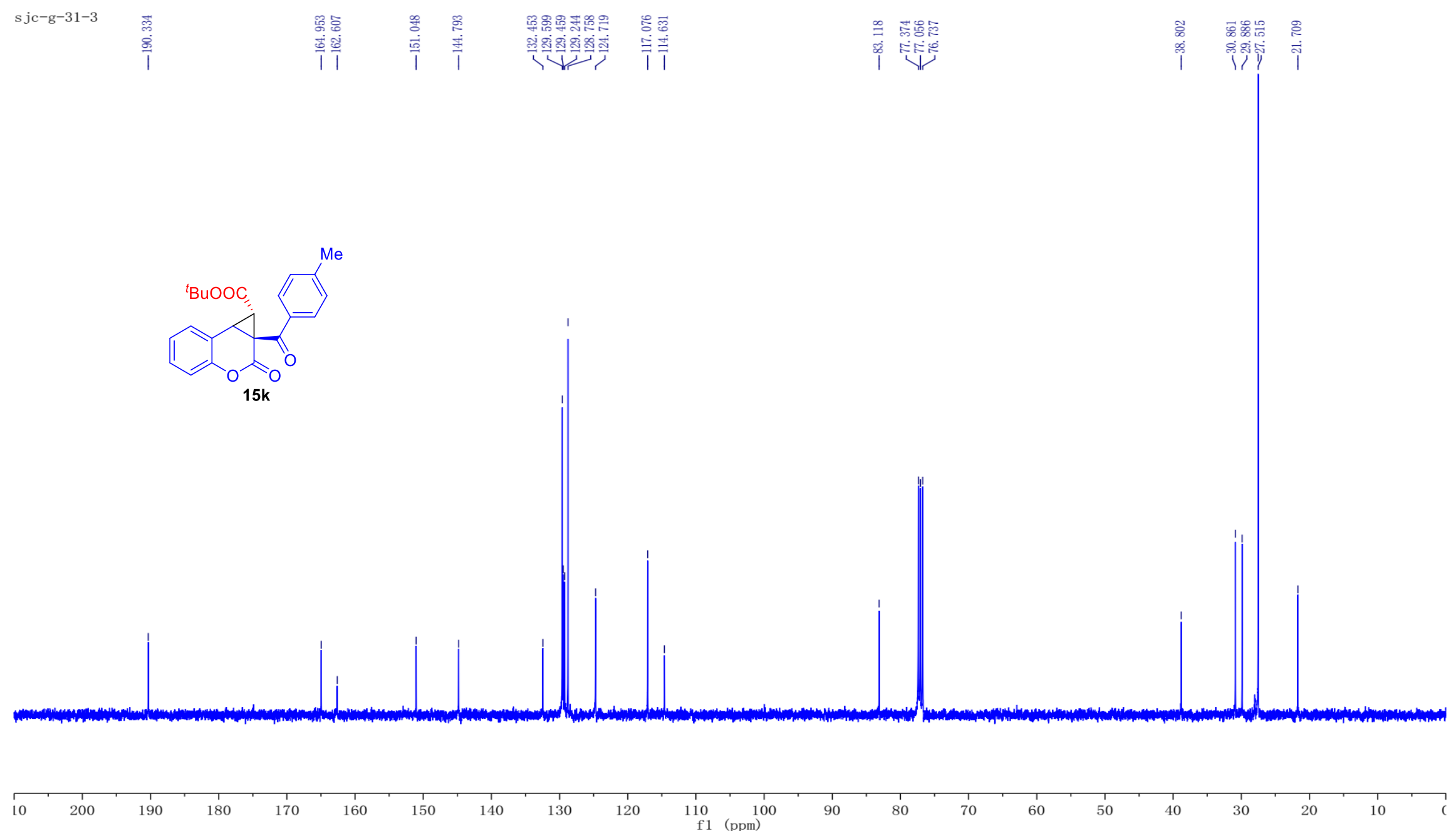
${ }^{1} \mathrm{H}$ NMR Spectrum (400 MHz, Chloroform- $d$ ) of $\mathbf{1 5 I}$

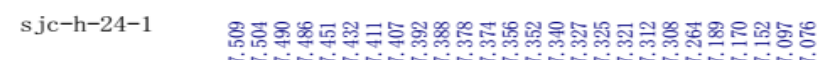

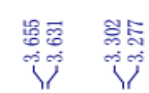

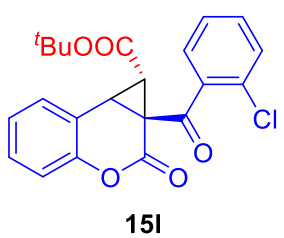

151

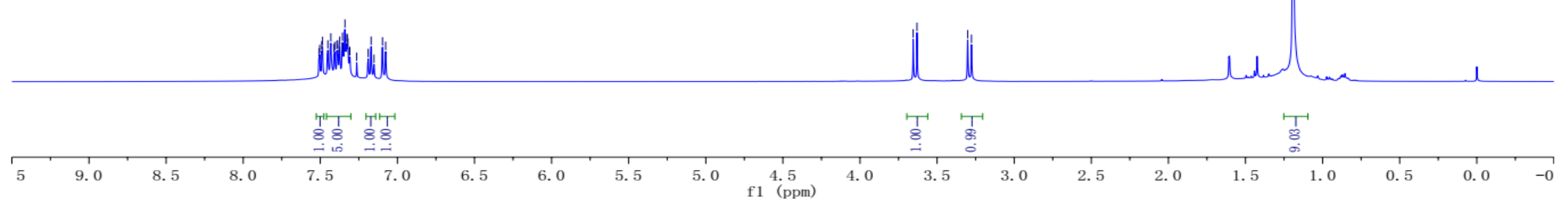


${ }^{13} \mathrm{C}\left\{{ }^{1} \mathrm{H}\right\}$ NMR Spectrum (101 MHz, Chloroform- $d$ ) of $\mathbf{1 5 l}$
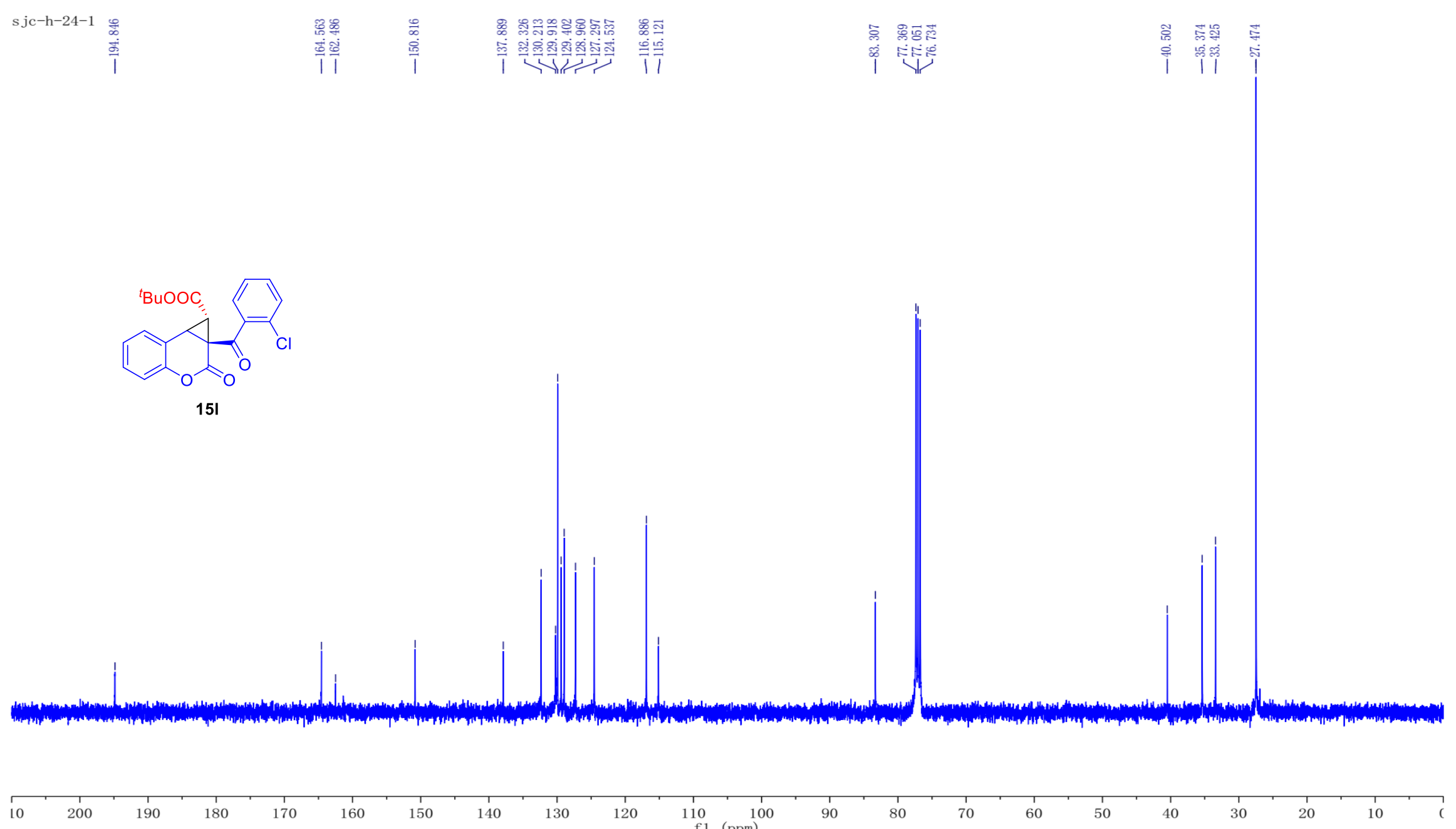
${ }^{1} \mathrm{H}$ NMR Spectrum (400 MHz, Chloroform- $d$ ) of $\mathbf{1 5 m}$

sjc-g-35-1-1

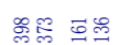

i j
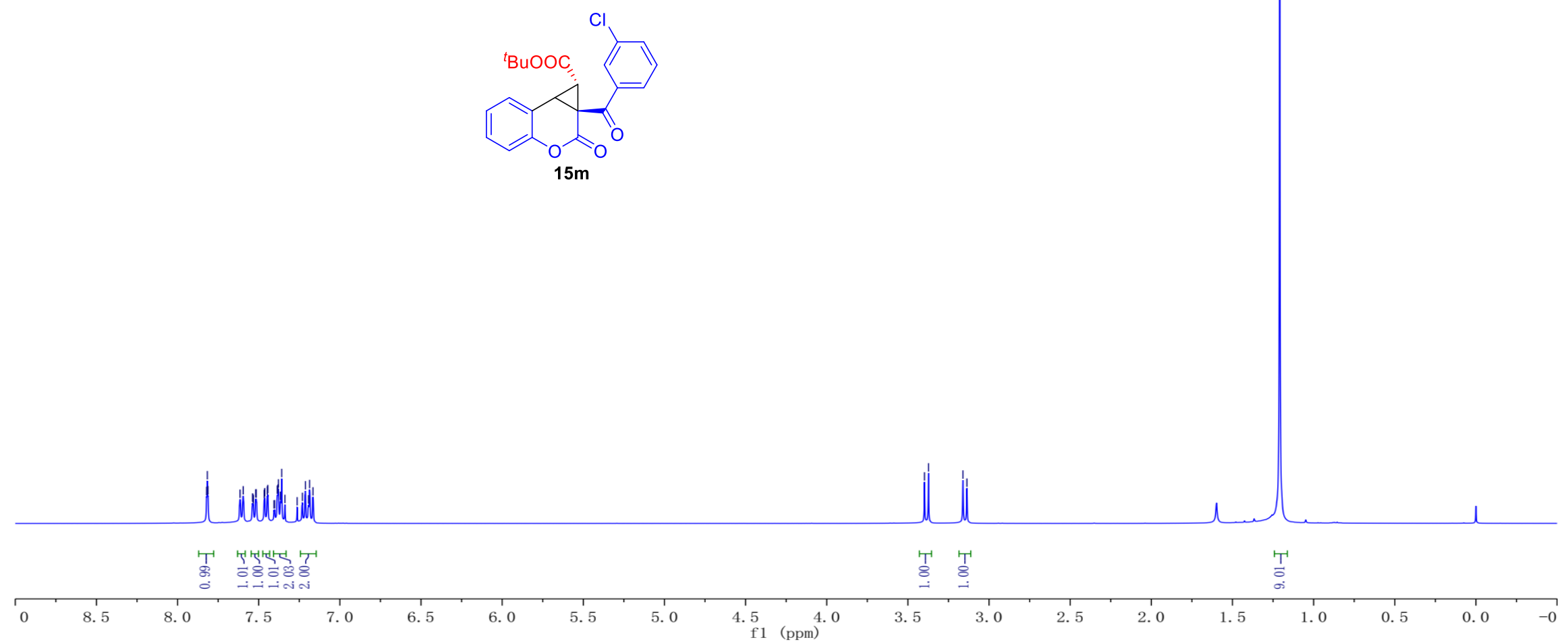
${ }^{13} \mathrm{C}\left\{{ }^{1} \mathrm{H}\right\}$ NMR Spectrum (101 MHz, Chloroform- $d$ ) of $\mathbf{1 5 m}$

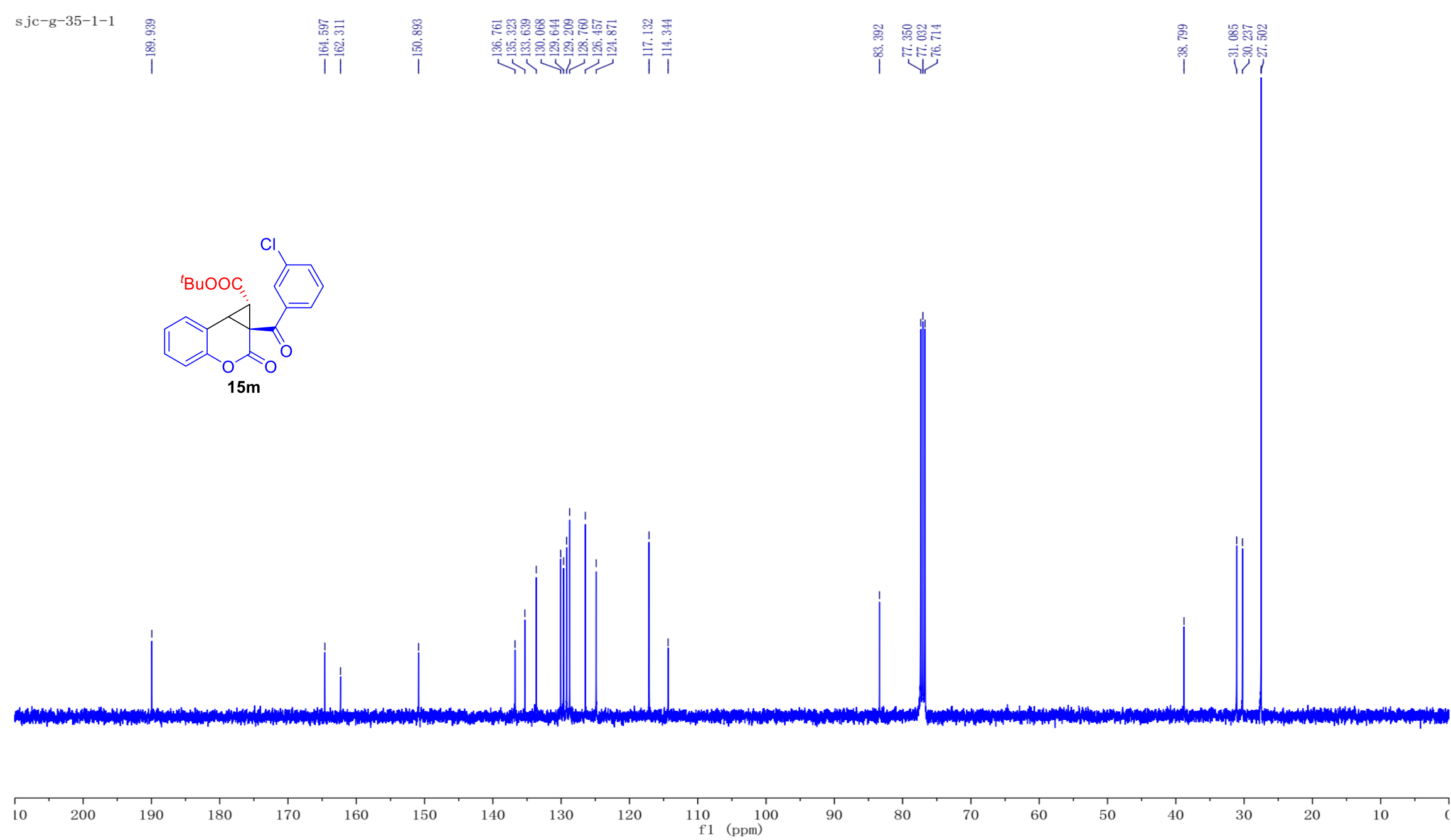

S54 
${ }^{1} \mathrm{H}$ NMR Spectrum (400 MHz, Chloroform- $d$ ) of $\mathbf{1 5 n}$

$\mathrm{sjc}-\mathrm{g}-35-2$

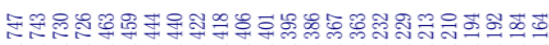

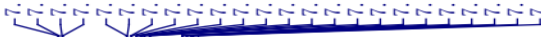

유ㅇㅛㅠ 웜

ip

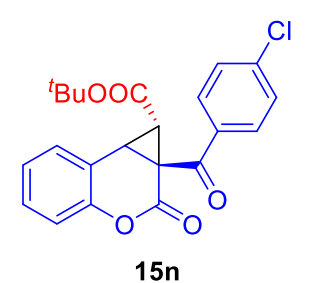

$15 n$

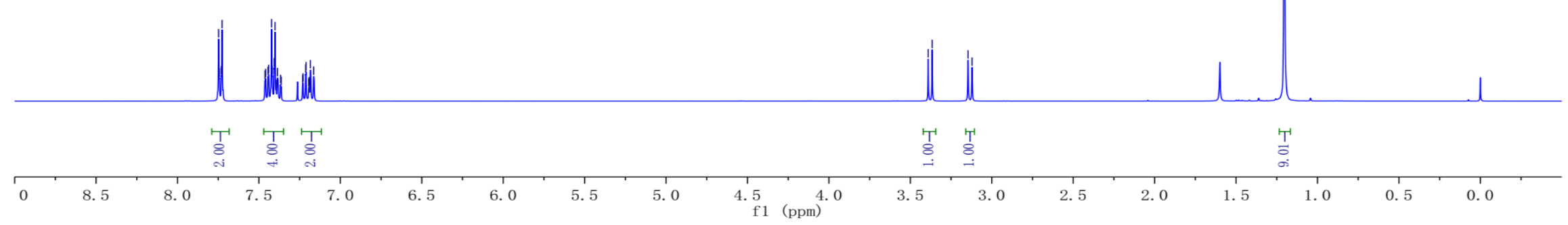

S55 
${ }^{13} \mathrm{C}\left\{{ }^{1} \mathrm{H}\right\}$ NMR Spectrum (101 MHz, Chloroform- $d$ ) of 15n
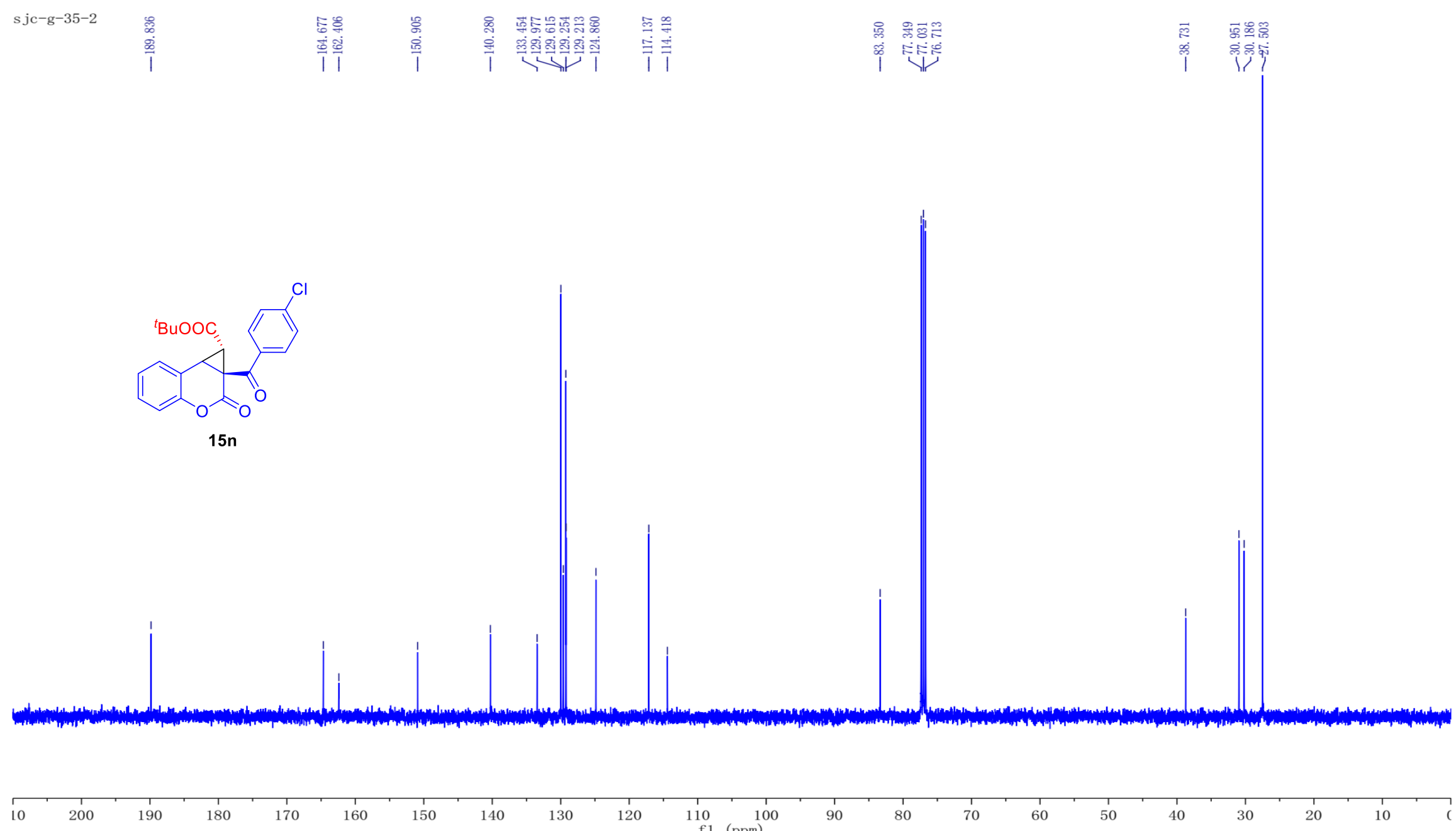
${ }^{1} \mathrm{H}$ NMR Spectrum (400 MHz, Chloroform- $d$ ) of $\mathbf{1 5 0}$

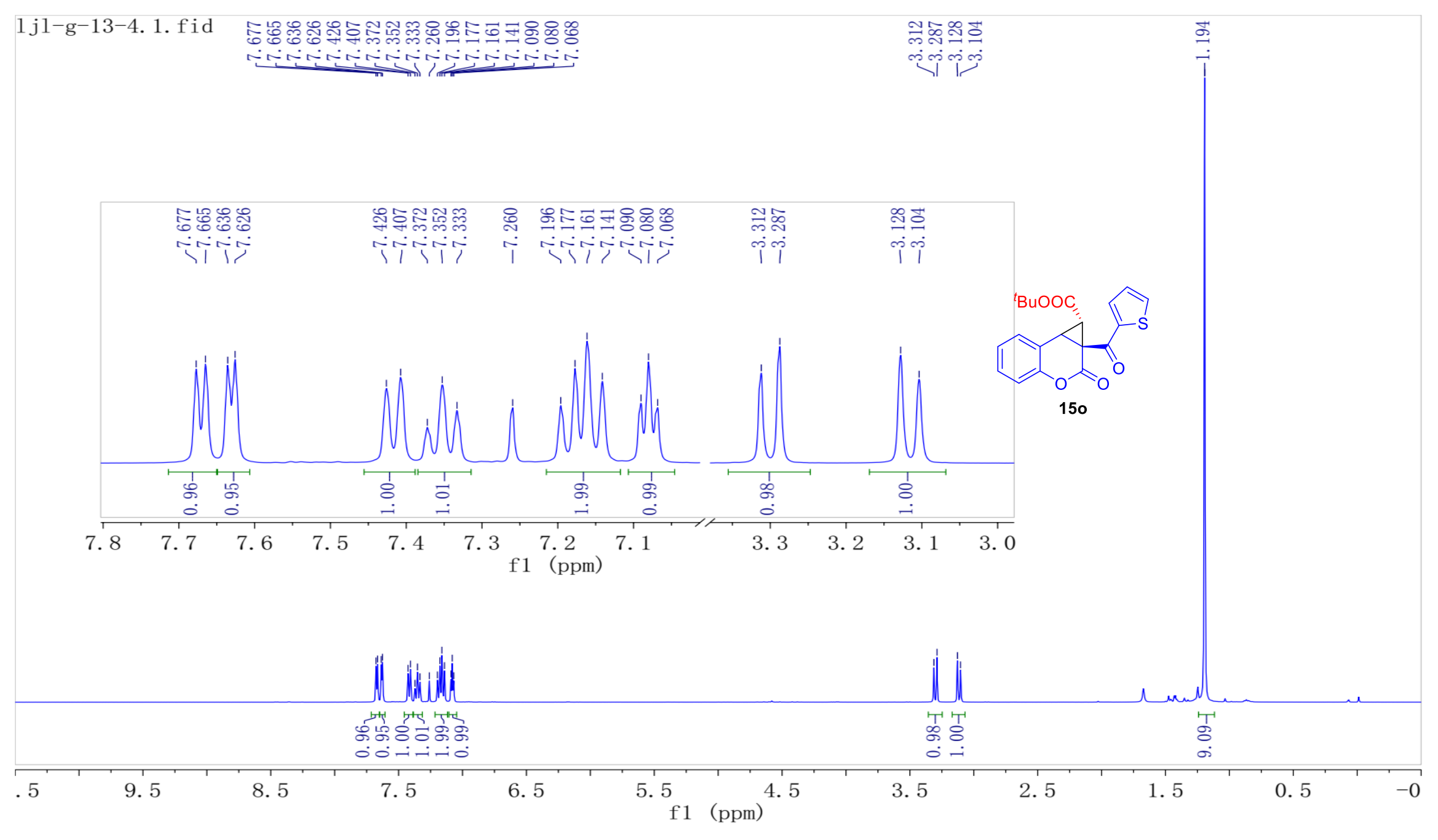


${ }^{13} \mathrm{C}\left\{{ }^{1} \mathrm{H}\right\}$ NMR Spectrum (101 MHz, Chloroform- $d$ ) of $\mathbf{1 5 o}$

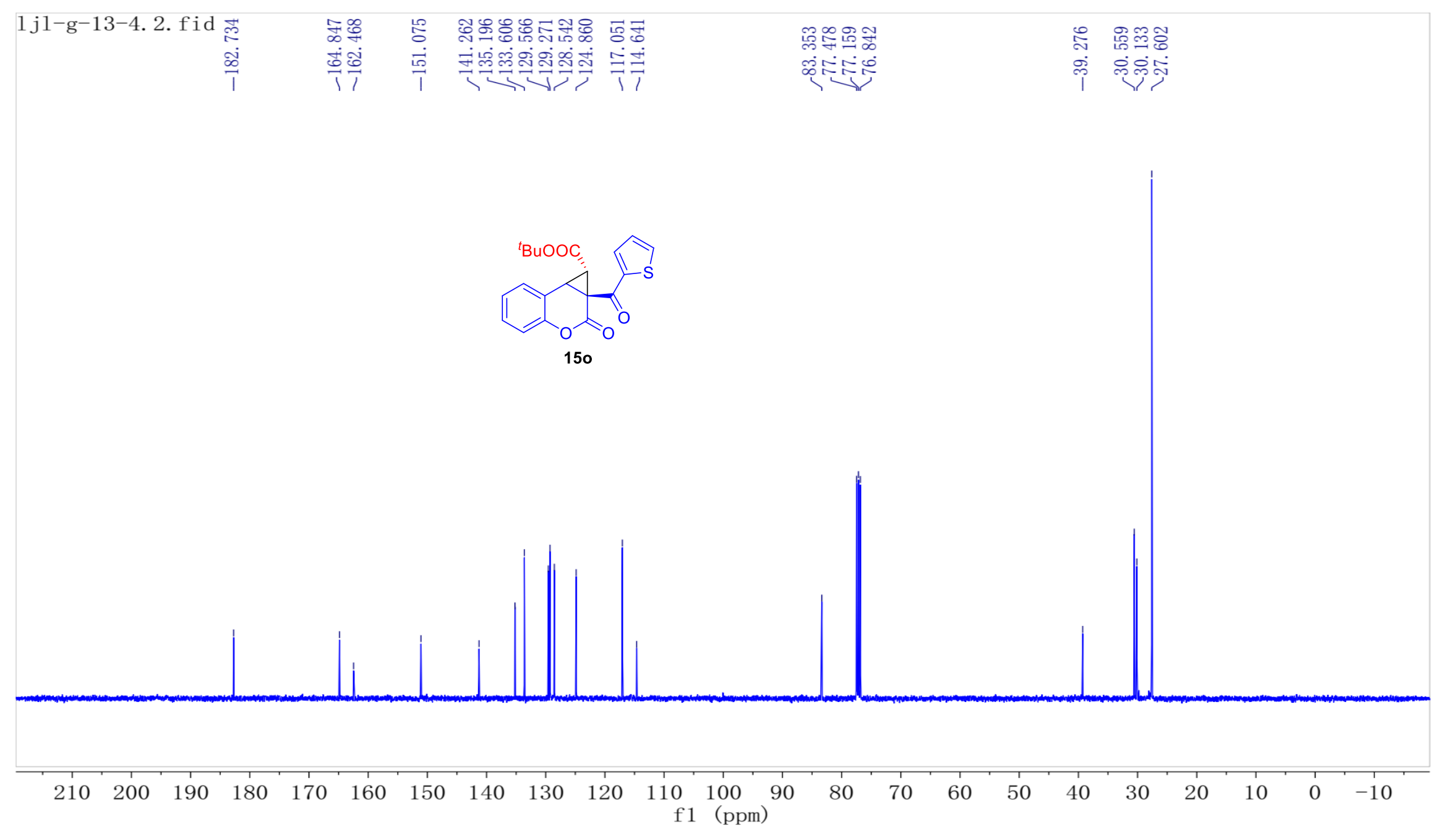




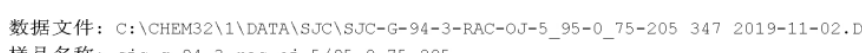
样品名称: sjc- $-94-3-\mathrm{rac}-0 j-5 / 95-0.75-205$
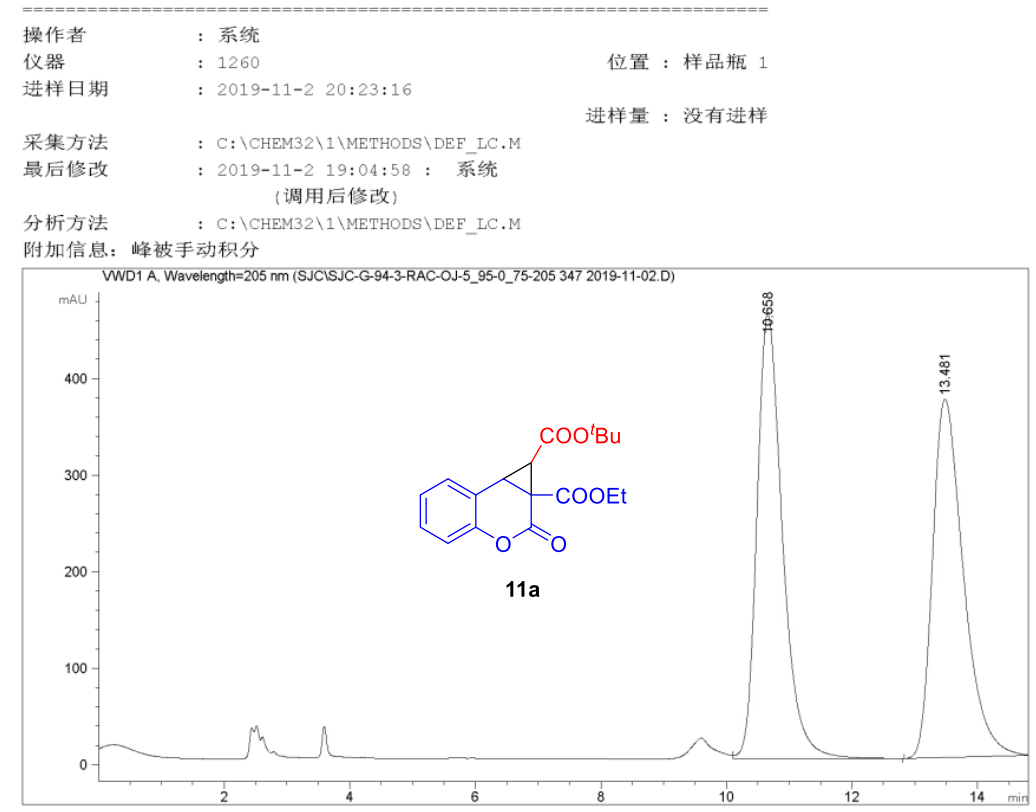

面积百分比报告

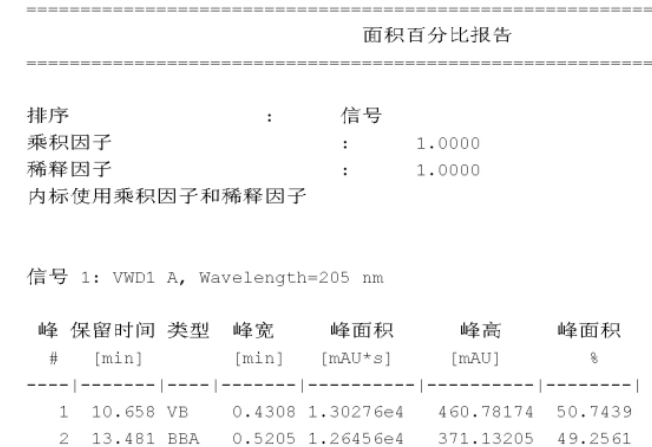

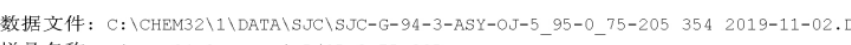

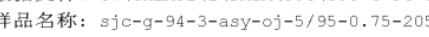

\begin{tabular}{|c|c|c|}
\hline \multirow{3}{*}{$\begin{array}{l}\text { 操作者 } \\
\text { 仪器 } \\
\text { 进样日期 }\end{array}$} & 系统 & \multirow{3}{*}{ 位置：样品瓶 1} \\
\hline & $: 1260$ & \\
\hline & : 2019-11-2 22:18:04 & \\
\hline $\begin{array}{l}\text { 采集方法 } \\
\text { 最后修改 }\end{array}$ & 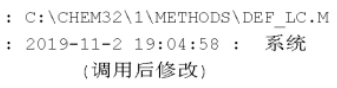 & \\
\hline 析方法 & $C: \backslash C H E M 32 \backslash 1 \backslash M E T H O D S \backslash D E F$ LC.M & \\
\hline
\end{tabular}
附加信息: 峰被手动积分

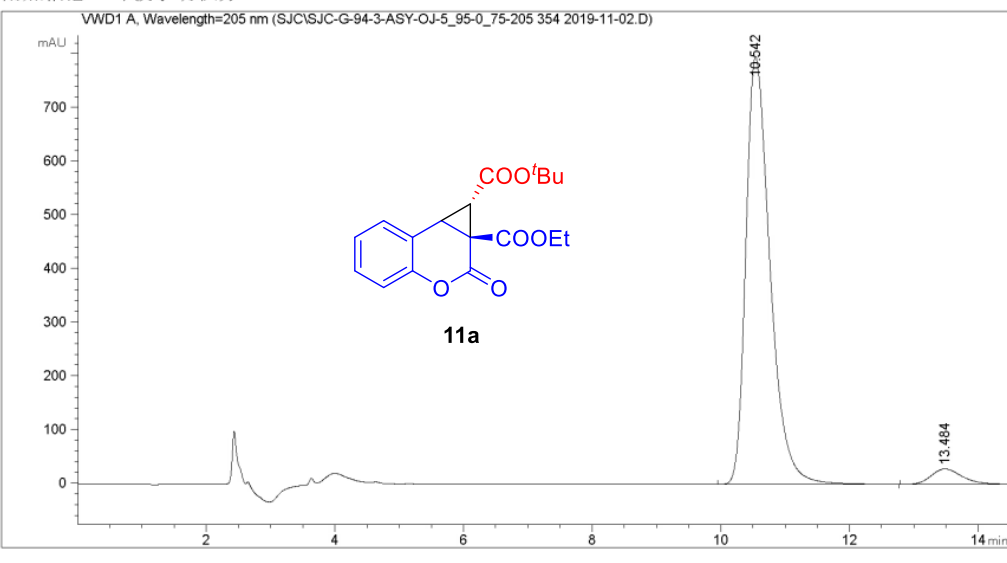

面积百分比报告

$\begin{array}{llll} & \text { 排序 } \\ \text { 乘积因子 } & \text { 信号 } & \\ \text { 稀稀因子 } & \vdots & 1.0000 \\ & : & 1.0000\end{array}$

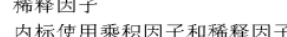

信号 1: VWD1 A, wavelength=205 nm

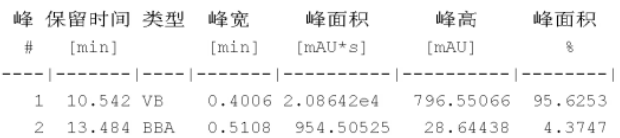




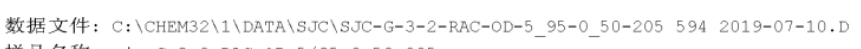
样品名称: sjo-G-3-2-PAC-OD-5/95-0.50-205

\begin{tabular}{|c|c|c|}
\hline 操作者 & : 系统 & \\
\hline 仪器 & $: 1260$ & 位置：样品瓶 1 \\
\hline 进样日期 & $: 2019-7-10 \quad 15: 45: 26$ & 进样量：没有进样 \\
\hline $\begin{array}{l}\text { 采集方法 } \\
\text { 最后修改 }\end{array}$ & 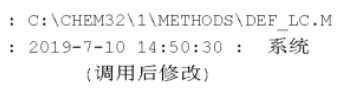 & \\
\hline 分析方法 & 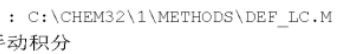 & \\
\hline
\end{tabular}

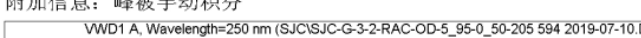

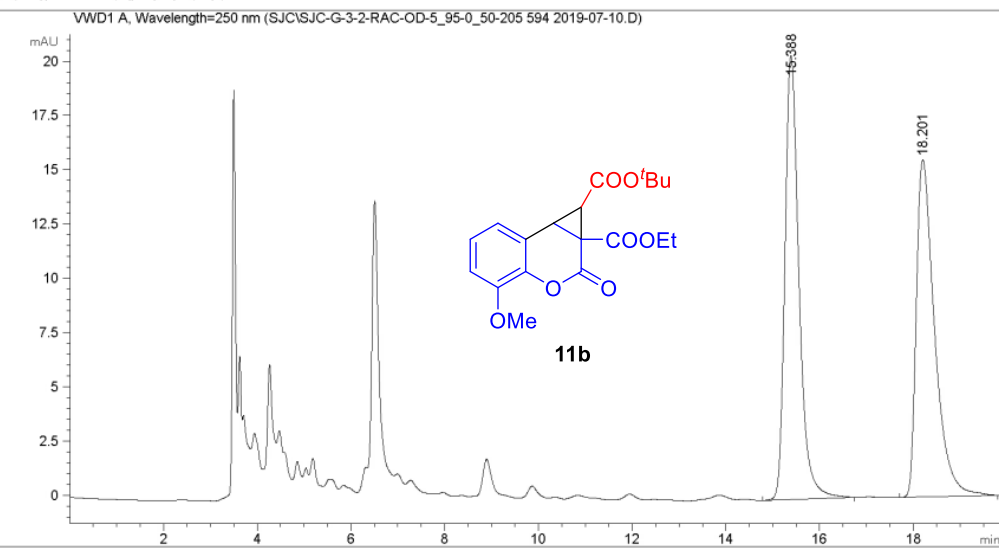

面积百分比报告

$\begin{array}{llll}\text { 排序 } & : & \text { 信号 } & \\ \text { 乘积因子 } & \vdots & 1.0000 \\ \text { 秝释因子 } & : & 1.0000\end{array}$

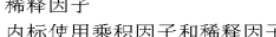

信号 1: VWD1 A, wavelength=250 nm

\begin{tabular}{|c|c|c|c|c|}
\hline $\begin{array}{l}\text { 保留时间 类型 } \\
\text { [min] }\end{array}$ & $\begin{array}{l}\text { 峰宽 } \\
\text { [min] }\end{array}$ & $\begin{array}{l}\text { 峰面积 } \\
{[\mathrm{mAU} * \text { * }]}\end{array}$ & $\begin{array}{l}\text { 峰高 } \\
\text { [mAU] }\end{array}$ & $\begin{array}{l}\text { 峰面积 } \\
8\end{array}$ \\
\hline & & & & \\
\hline
\end{tabular}

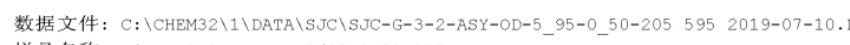
样品名称:

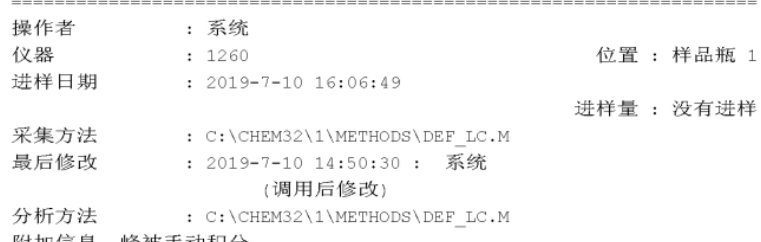

分析方法 $:$ : : \CHEM32\1 1 METHODS $\backslash$ DEF_LC.

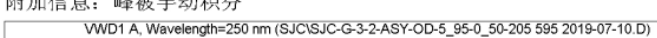

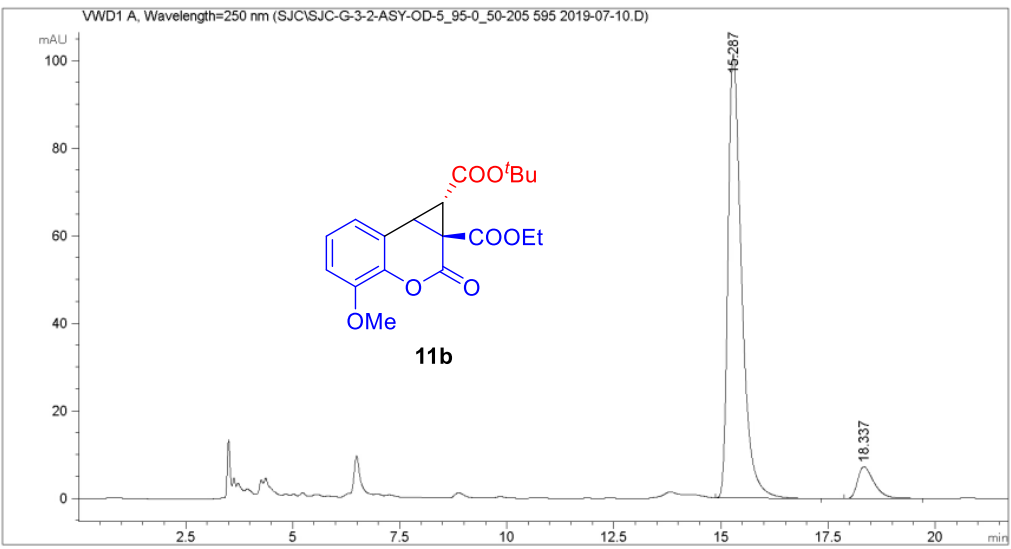

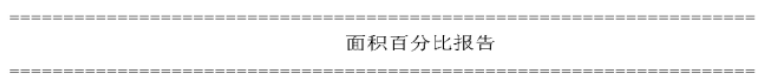

排序

信号

乘积因子

稀释因子

内标使用乘积因子和稀皕因子

信号 1: VWD1 A, wavelength=250 nm

峰保留时间 类型峰宽峰面积峰高峰面积

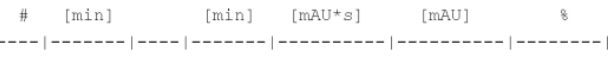

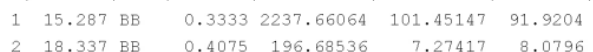

1260 2019-7-10 16:45:16 系统 


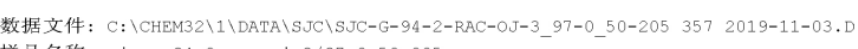
羊品名称: sjc-g-94-2-rac-oj-3/97-0.50-20

\begin{tabular}{|c|c|c|}
\hline \multirow{3}{*}{$\begin{array}{l}\text { 操作者 } \\
\text { 进样日期 }\end{array}$} & : 系统 & \\
\hline & $: 1260$ & 位置：样品瓶 1 \\
\hline & : 2019-11-3 12:19:51 & 进样量：没有进样 \\
\hline 采集方: & 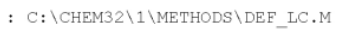 & \\
\hline 最后修改 & $\begin{array}{c}\text { ：2019-11-3 12:02:39： 系统 } \\
\text { (调用后修改) }\end{array}$ & \\
\hline 分析方法 & $\begin{array}{l}: C: \backslash C H E M 32 \backslash 1 \backslash M E T H O D S \backslash D E E \_L C . M \\
: \text { 动积分 }\end{array}$ & \\
\hline
\end{tabular}

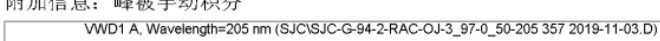

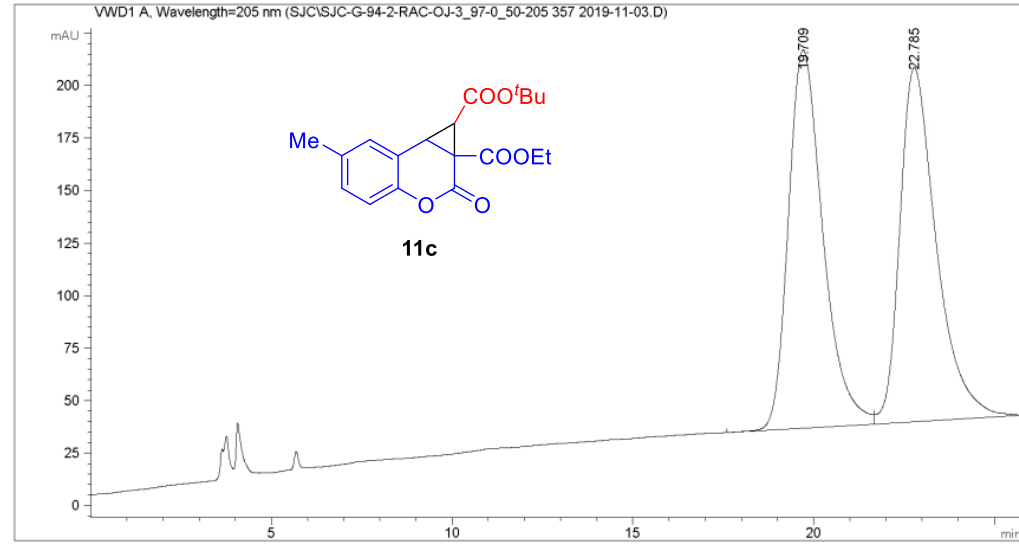

面积百分比报告

$\begin{array}{llll}\text { 排序 } & : & \text { 信号 } & \\ \text { 乘积因子 } & \vdots & 1.0000 \\ \text { 桸勫因子 } & : & 1.0000\end{array}$

内标使用乘积因子和称释因子

信号 1: VWD1 A, wavelength=205 $\mathrm{nm}$

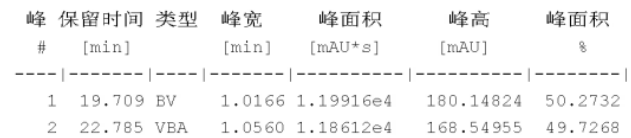

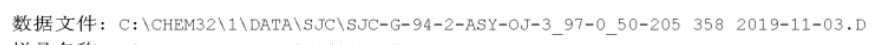
样品名称: sjc-q-94-2-asy-oj-3/97-0.50-205
操作者系线
位置：样品瓶

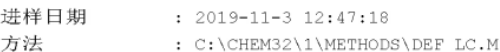 附加信息：峰被手动积分

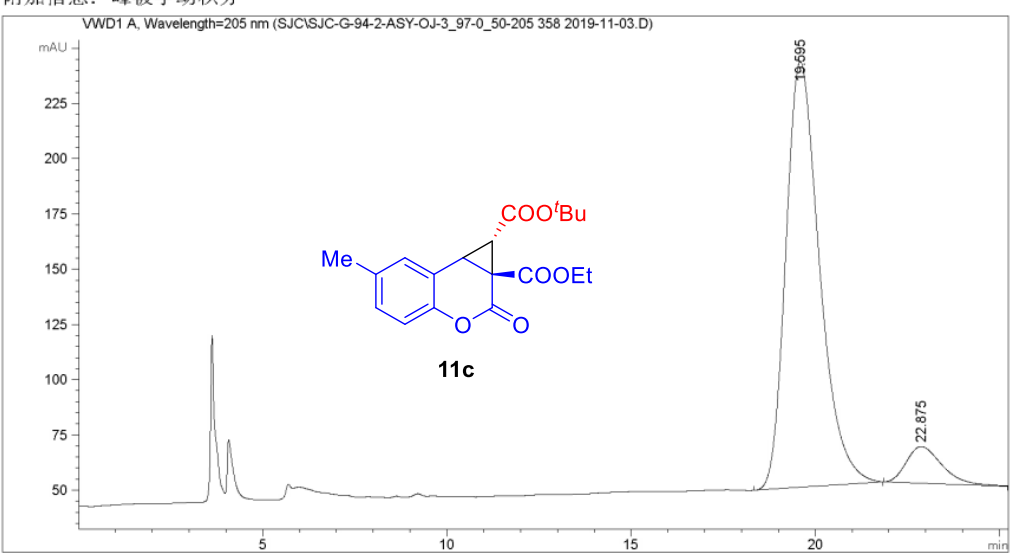

面积百分比报告

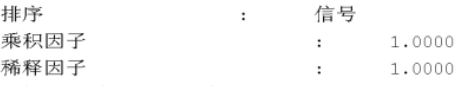

内稀释因子

言号 1: VWD1 A, Wavelength=20.5 $\mathrm{mm}$

峰保留时间类型峰宽峰面积峰高峰面积

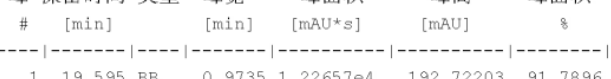

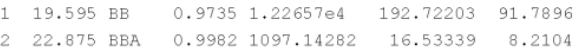

总量 : $\quad 1.33629 e 4 \quad 209.2554$

1260 2019-11-3 13:14:14 系纯

页 $1 / 1$ 


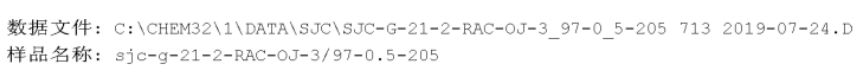

\begin{tabular}{|c|c|c|}
\hline \multirow{3}{*}{$\begin{array}{l}\text { 操作者 } \\
\text { 仪器 } \\
\text { 进样日期 }\end{array}$} & 系统 & \\
\hline & $: 1260$ & 位置：样品瓶 1 \\
\hline & $: 2019-7-24 \quad 20: 50: 44$ & \\
\hline 采集方法 & : C: $\backslash$ CHEM $2 \backslash \backslash \backslash$ METHODS $\backslash D E E_{-}$LC.M & \\
\hline 最后修改 & ：2019-7-24 19:59:09：系统 & \\
\hline & $\begin{array}{c}\text { (调用后修改) } \\
C: \backslash C H E 32 \backslash 1 \backslash M E T H O D S \backslash D E F \text { LC.M }\end{array}$ & \\
\hline
\end{tabular}

附加信息: 峰被手动积分

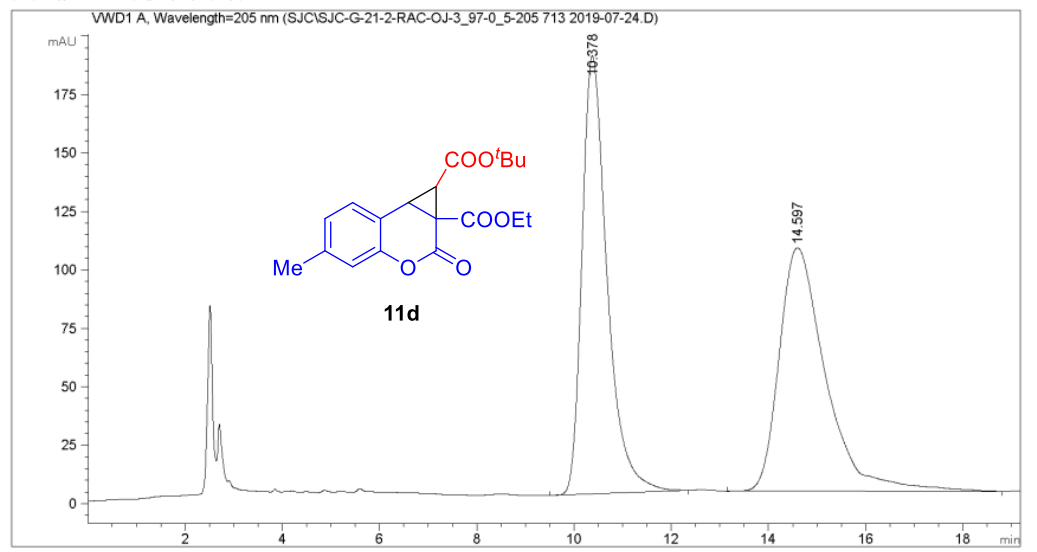

面积百分比报告

$\begin{array}{llll}\text { 排序 } & : & \text { 信号 } & \\ \text { 乘积因子 } & \vdots & 1.0000 \\ \text { 稍释因子 } & : & 1.0000\end{array}$

稀释因子

信号 1: VWD1 A, wavelength=205 nm

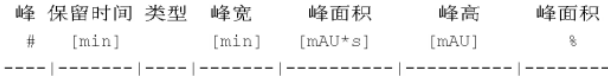

$\begin{array}{llllll}1 & 10.378 \text { вв } \quad 0.5446 & 6737.14258 & 187.31308 & 49.3654\end{array}$

1260 2019-7-25 21:17:04 系统 数据文件: C: \CHEM32\1 \DATA \SJC\SJC-G-21-2-ASY-OJ-3_97-0_5-205 714 2019-07-24.D

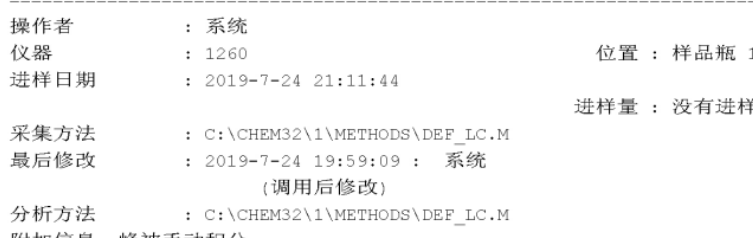

分析方法 : C : \CHEM32\1 \METHODS \DEF_LC.

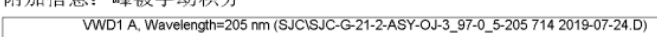

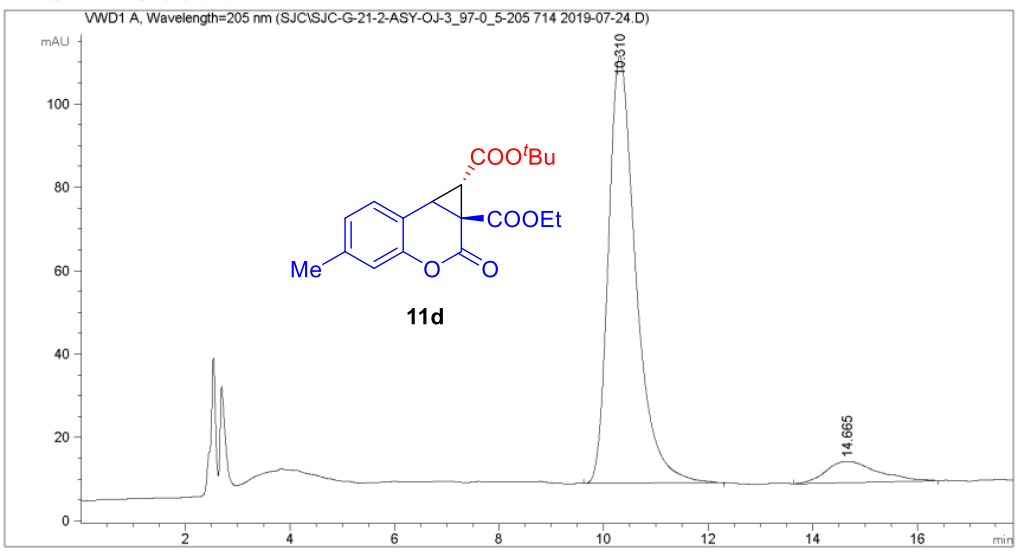

面积百分比报告

排序积因子

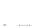

$: \quad 1.0000$

等和稀释因子

信号 1: VWD1 A, Wavelength=205 nm

峰保留时间类型峰宽峰面积峰高峰面积

\# $[\mathrm{min}] \quad[\mathrm{min}] \quad\left[\mathrm{mAU} \mathrm{N}^{\mathrm{s}}\right] \quad$ [mAU] 8

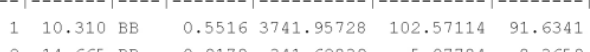

2019-7-25 21:17:21 系统 


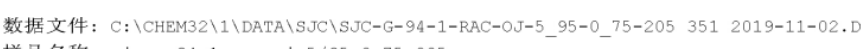
羊品名称: sjc-g-94-1-rac-oj-5/95-0.75-20

\begin{tabular}{|c|c|c|}
\hline 操作者 & : 系统 & \\
\hline 仪器 & $: 1260$ & 位置：样品瓶 \\
\hline 进样日期 & $: 2019-11-2 \quad 21: 31: 08$ & \\
\hline 采集方法 & $: \mathrm{C}: \backslash \mathrm{CHEM} 32 \backslash 1 \backslash \mathrm{METHODS} \backslash \mathrm{DEF}_{\mathrm{C}} \mathrm{LC} . \mathrm{M}$ & \\
\hline 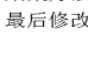 & $\begin{array}{l}\text { : 2019-11-2 19:04:58： 系统 } \\
\text { (调用后修改) }\end{array}$ & \\
\hline 分析方法 & $: C: \backslash C H E M 32 \backslash 1 \backslash M E T H O D S \backslash D E E_{-}$LC.M & \\
\hline
\end{tabular}

附加信息: 峰被手动积分

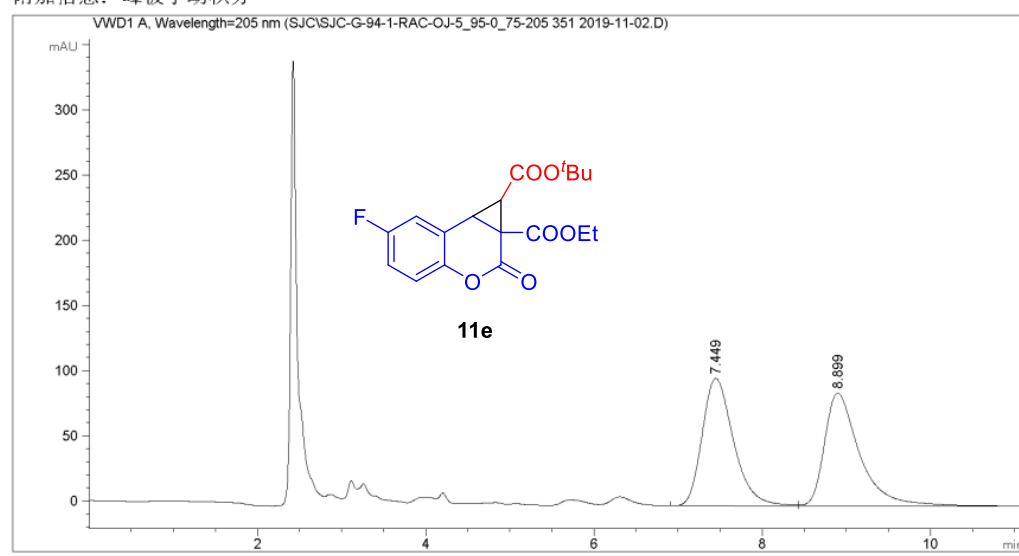

面积百分比报告

$$
\text { 排序 }
$$

乘积因子

$\begin{array}{lll}\text { 乘积因子 } & : & 1.0000 \\ \text { 稀秚因子 } & : & 1.0000 \\ \text { 内标使用乘积因子和稀释因子 } & & \end{array}$

信号 1: VWD1 A, wavelength=205 nm

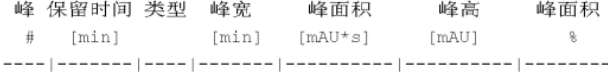

$\begin{array}{llllll}1 & 7.449 \mathrm{BV} & 0.3975 & 2534.69971 & 97.75158 & 50.3265\end{array}$

1260 2019-11-3 11:08:39 系统

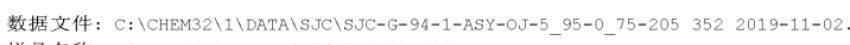

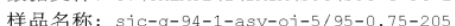

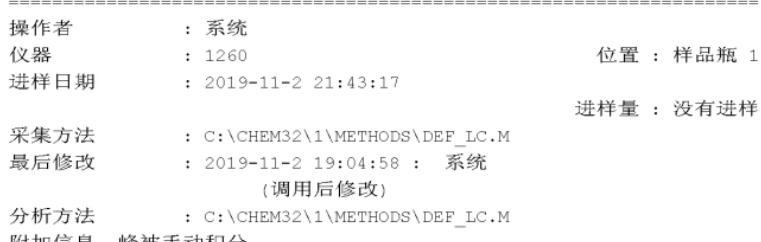

分析方法 $\quad$ C : \CHEM32\1 \METHODS \DEF_LC. .

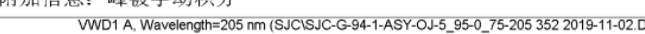

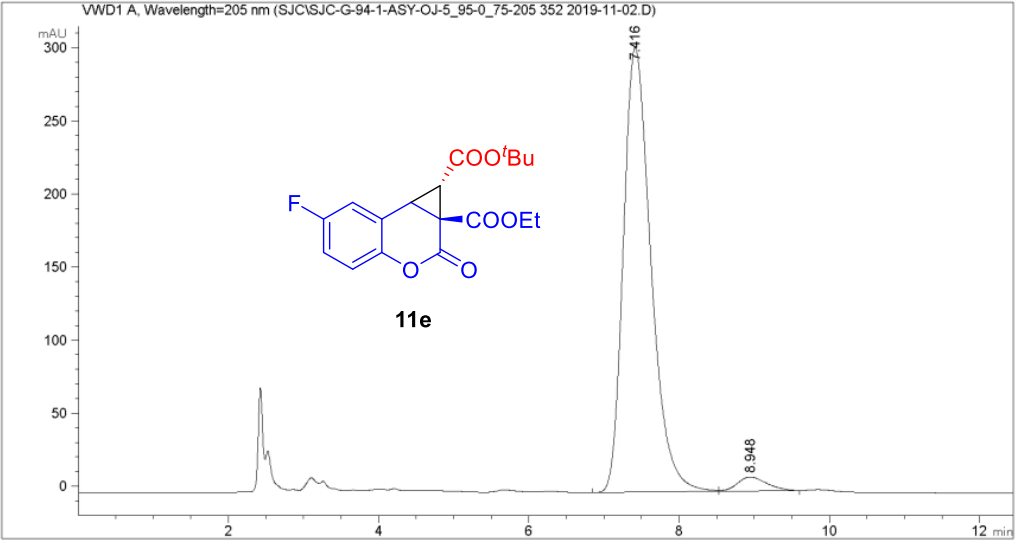

面积百分比报告

排序

乘积因子

信号

$\begin{array}{ll}: & 1.0000 \\ \vdots & 1.0000\end{array}$

信号 1: VWD1 A, Wavelength=205

峰保留时间 类型峰宽峰面积峰高峰面积

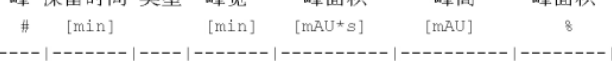

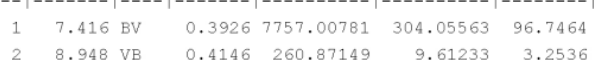

1260 2019-11-3 11:09:20 系统 


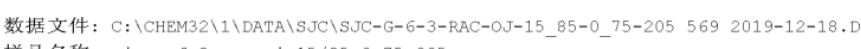
羊品名称: sjc- $9-6-3-\mathrm{rac}-0 j-15 / 85-0.75-20$

\begin{tabular}{|c|c|c|}
\hline \multirow{2}{*}{$\begin{array}{l}\text { 操作者 } \\
\text { 仪器 }\end{array}$} & & \multirow{2}{*}{ 位置：样品瓶 1} \\
\hline & $\begin{array}{l}1 \\
: 1260\end{array}$ & \\
\hline 进样日期 & $: 2019-12-18 \quad 20: 14: 57$ & \\
\hline 采集方法 & : C: $\backslash$ CHEM32\} 1 \backslash \text { METHODS } \backslash T E S T , M $&{\text { 进样量：没有进样 }} \\
{\hline \text { 最后修改 }} &{\begin{array}{l}\text { : 2019-12-18 19:32:00： 系统 } \\
\text { (调用后修改) }\end{array}} &{ } \\
{\hline \text { 析方 }} &{\text { 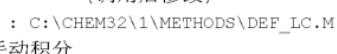 }} &{ } \\
$\hline
\end{tabular}

附加信息：峰被手动积分

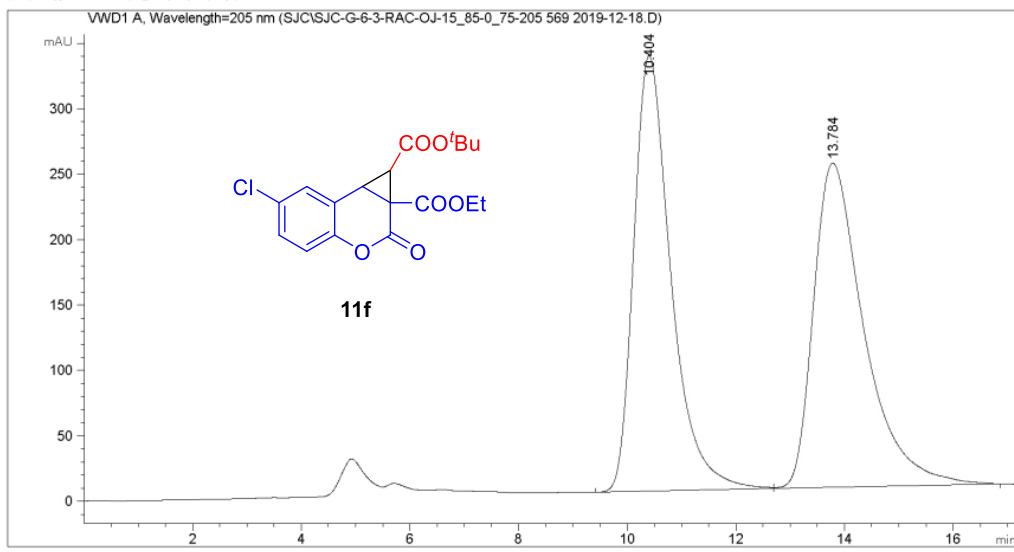

面积百分比报告

$\begin{array}{llll}\text { 排序 } & : & \text { 信号 } & \\ \text { 乘积因子 } & \vdots & 1.0000 \\ \text { 稍释因子 } & : & 1.0000\end{array}$

稀释因子

信号 1: VWD1 A, wavelength=205 nm

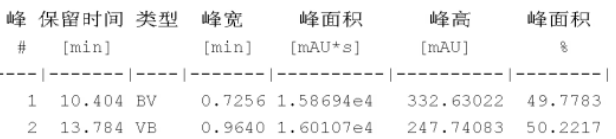
数据文件: C: \CHEM32\1 \DATA \SJC\SJC-G-6-3-ASY-OJ-15_85-0_75-205 571 2019-12-18.D

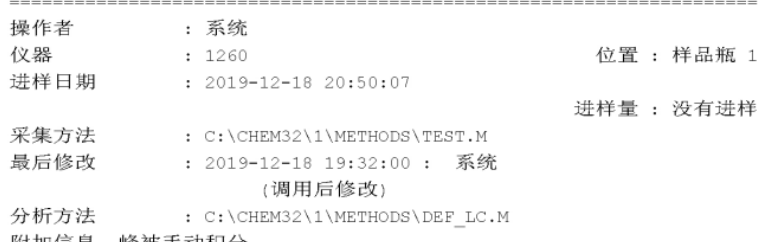

分析方法 : C : \CHEM32\1 \METHODS \DEE_LC.

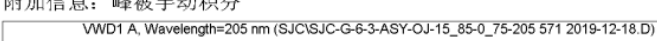

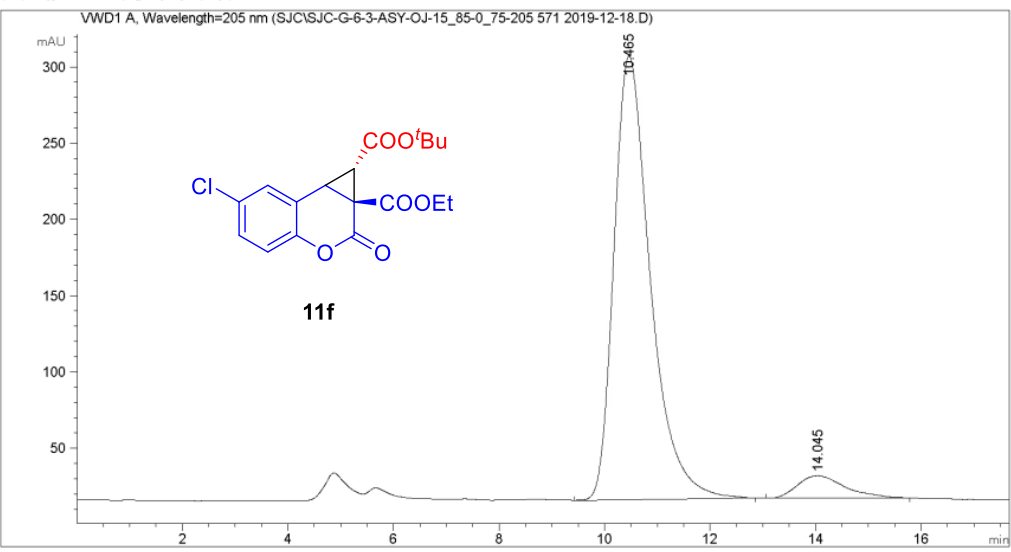

面积百分比报告

排序积因子

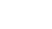

希释因子

: 1.0000

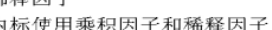

信号 1: VWD1 A, wavelength=205 nm

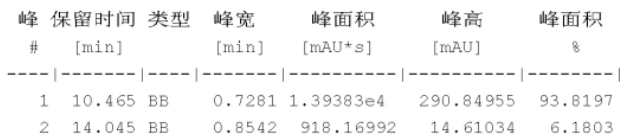




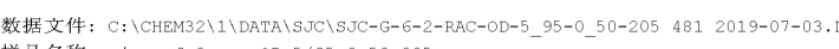
羊品名称: sjc- $9-6-2-2 a c-0 D-5 / 95-0.50-205$

\begin{tabular}{|c|c|c|}
\hline 操作者 & : 系统 & \\
\hline & $: 1260$ & 位置：样品瓶 1 \\
\hline 进样日期 & $: 2019-7-3 \quad 20: 08: 29$ & 进样量：没有进样 \\
\hline $\begin{array}{l}\text { 采集方法 } \\
\text { 最后修改 }\end{array}$ & 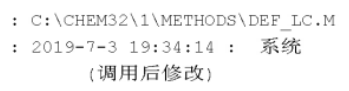 & \\
\hline $\begin{array}{l}\text { 分析方法 } \\
\text { 附加信息: }\end{array}$ & $\begin{array}{l}\text { C: C \CHEM32\1 } \backslash \text { METHODS \DEE_LC.M } \\
\text { 动积分 }\end{array}$ & \\
\hline
\end{tabular}

附加信息: 峰被手动积分

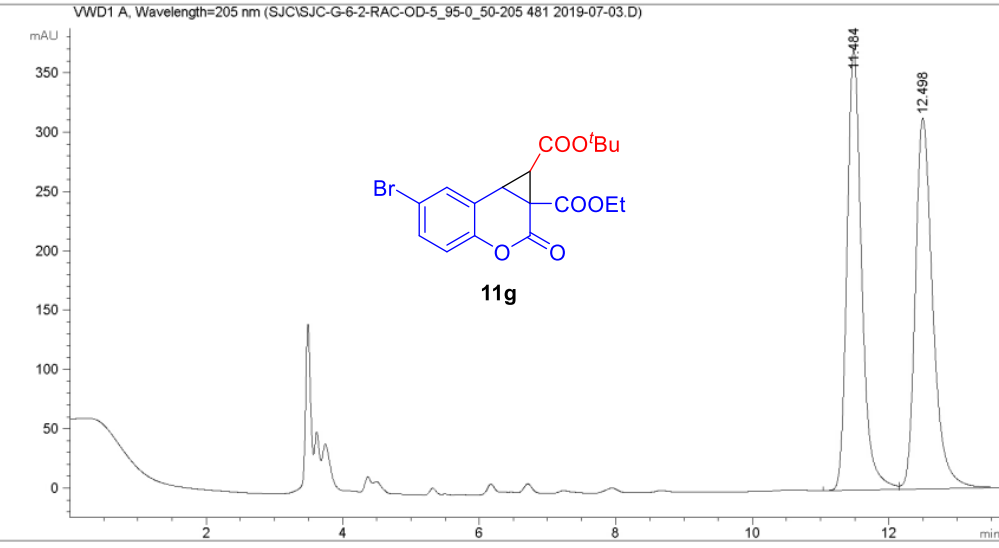

面积百分比报告

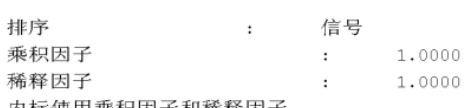

内标值用乘和因子和残释团

信号 1: VWD1 A, wavelength=205 nm

\section{峰保留时间类型峰宽峰面积峰高峰面积}

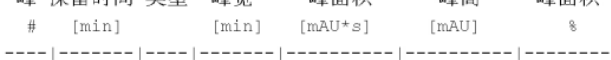

$\begin{array}{llllll}1 & 11.484 \text { BV } & 0.2282 & 5537.42920 & 370.87085 & 49.9337\end{array}$
数据文件: C: \CHEM32\1\DATA \SJC \SJC-G-6-2-ASY-OD-5_95-0_50-205482 2019-07-03.D

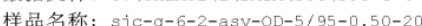

\begin{tabular}{|c|c|c|}
\hline 操作者 & : 素统 & \\
\hline 仪器 & $: 1260$ & 位置：样品瓶 1 \\
\hline 进样日期 & $: 2019-7-3 \quad 20: 24: 02$ & \\
\hline 采集方法 & 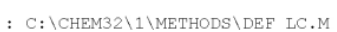 & \\
\hline 最后修改 & $\begin{array}{r}\text { : 2019-7-3 19:34:14：系统 } \\
\text { (调用后修改) }\end{array}$ & \\
\hline
\end{tabular}

分析方法 $:$ : : \CHEM32 $21 \backslash$ METHODS $\backslash$ DEF_LC.

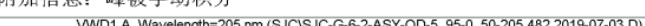

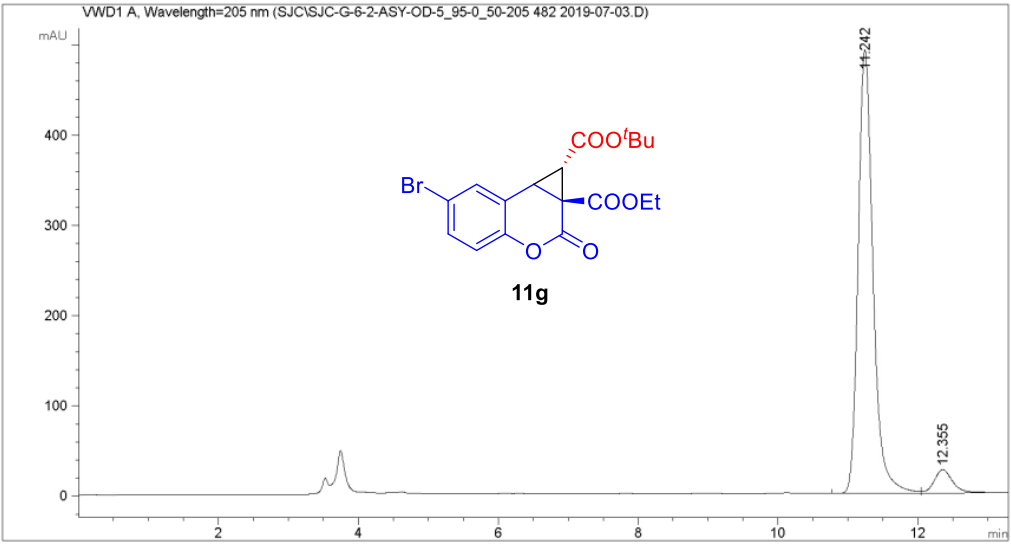

面积百分比报告

排序

(1)

乘积因子

希释因子

用乘积因子和楼释因子

信号 1: VWD1 A, Wavelength=205 nir

峰保留时间 类型峰宽峰面积峰高峰面积

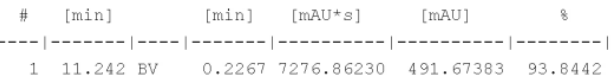

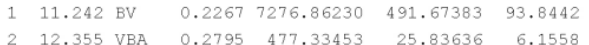

1260 2019-7-3 20:40:39 系统 


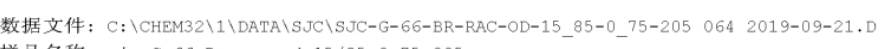
样品名称: sic- G- 6 - $-\mathrm{B} r-\mathrm{rac}-\mathrm{od}-15 / 85-0.75-205$

\begin{tabular}{|c|c|c|}
\hline \multirow{3}{*}{$\begin{array}{l}\text { 操作者 } \\
\text { 进样日期 }\end{array}$} & \\
\hline & 1260 & 位置：样品瓶 1 \\
\hline & $: 2019-9-21 \quad 16: 46: 21$ & \\
\hline 采集方法 & : C:।CHEM $2 \backslash 1 \backslash$ METHODS $\backslash D E F$ LC.M & 没有进杽 \\
\hline 最后修改 & $\begin{array}{l}: 2019-9-2115: 02: 09: \text { 䇣统 } \\
\text { (调用后修改) }\end{array}$ & \\
\hline 分析方法 & $\mathrm{C}: \backslash \mathrm{CHEM} 32 \backslash 1 \mathrm{METHODS} \backslash \mathrm{DEF}$ _LC.M & \\
\hline 扣信息: & 只分 & \\
\hline
\end{tabular}

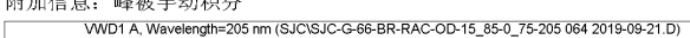

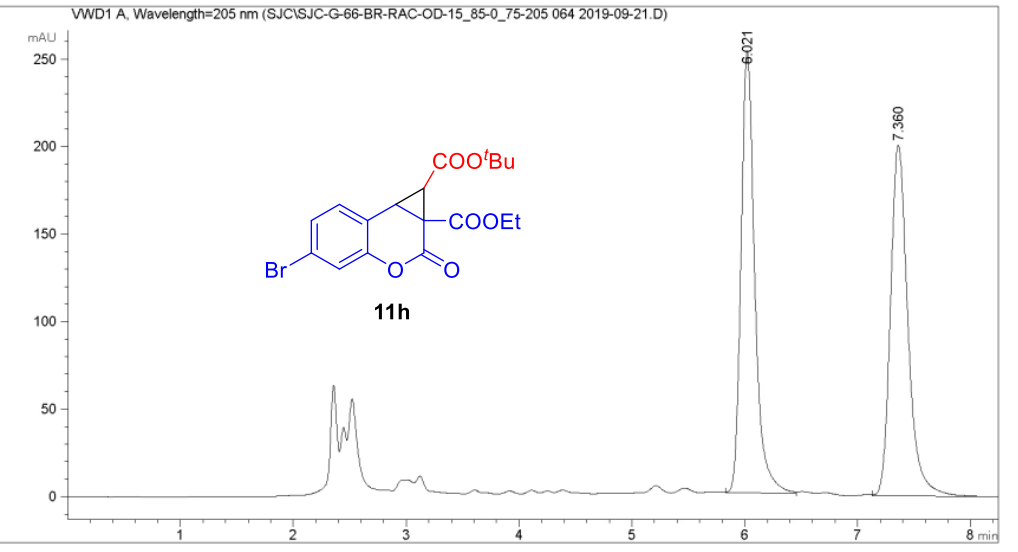

面积百分比报告

$\begin{array}{llll}\text { 排序 } & : & \text { 信号 } & \\ \text { 乘积因子 } & \vdots & 1.0000 \\ \text { 饰䉽因子 } & : & 1.0000\end{array}$

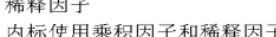

信号 1: VWD1 A, wavelength=205 nm

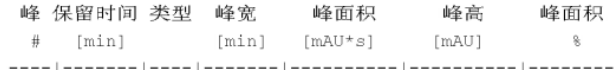

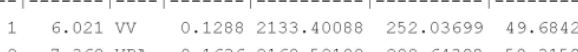

1260 2019-9-21 21:02:50 系统

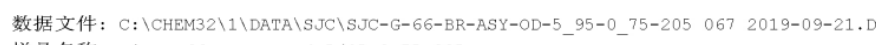
样品名称, sjc-0 - $66-\mathrm{B} x-2.9 \mathrm{y}-0 \mathrm{~d}-5 / 95-0.75-205$

\begin{tabular}{|c|c|c|}
\hline 操作者 & : 系统 & \\
\hline 仪器 & $: 1260$ & 位置：样品瓶 1 \\
\hline 进样日期 & $:$ 2019-9-21 17:21:31 & \\
\hline 采集方法 & 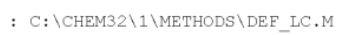 & \\
\hline 最后修改 & $\begin{array}{c}\text { : 2019-9-21 15:02:09： 系统 } \\
\text { (调用后修改) }\end{array}$ & \\
\hline 析方法 & 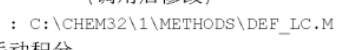 & \\
\hline
\end{tabular}

Woraw

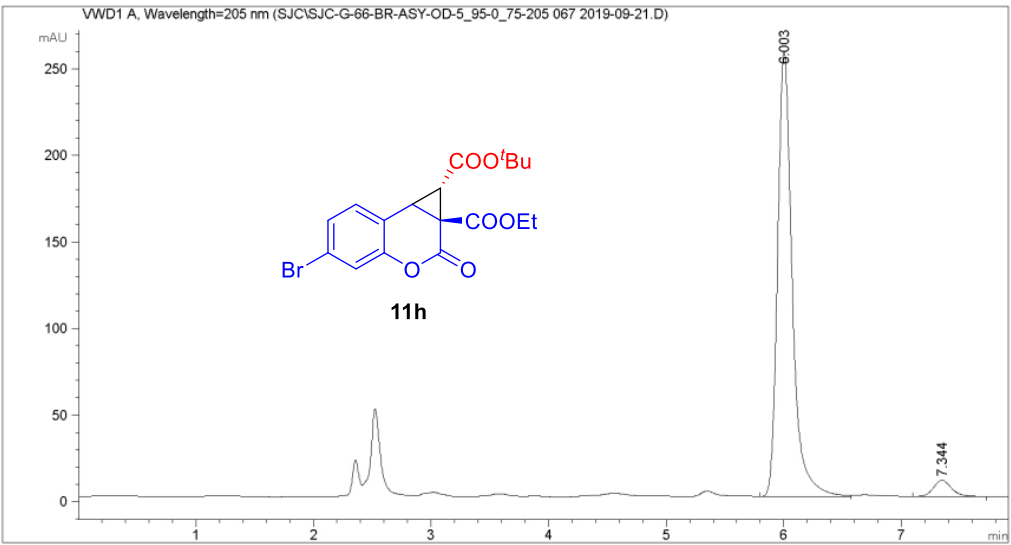

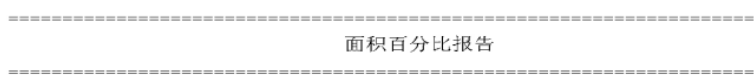

排序

信号

乘积因子

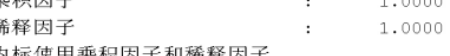

信号 1: VWD1 A, Wavelength=205 $\mathrm{nr}$

峰保留时间类型峰宽峰面积峰高峰面积

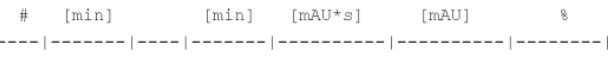

$\begin{array}{lrrrrr}1 & 6.003 \mathrm{BV} & 0.1321 & 2224.43799 & 256.75482 & 95.7774 \\ 2 & 7.344 \mathrm{BB} & 0.1610 & 98.06953 & 9.30361 & 4.2226\end{array}$

1260 2019-9-21 21:03:04 系统 


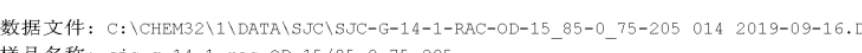
羊品名称: sic- $q$ - $14-1-\mathrm{rac}-\mathrm{OD}-15 / 85-0.75-205$

\begin{tabular}{|c|c|c|}
\hline \multirow{2}{*}{$\begin{array}{l}\text { 操作者 } \\
\text { 仪器 }\end{array}$} & 系统 & \multirow{2}{*}{ 位置：样品瓶 1} \\
\hline & $\therefore 1260$ & \\
\hline & $: 2019-9-16 \quad 15: 42: 30$ & \\
\hline 采集方法 & : C: $\backslash$ CHЕМ $2 \backslash 1 \backslash$ METHODS $\backslash D E F$ LC.M & 进样量：没有进样 \\
\hline $\begin{array}{l}\text { 最后修改 } \\
\text { 等 }\end{array}$ & : 2019-9-16 15:27:16 : 系统 & \\
\hline & $\begin{array}{c}\text { (调用后修改) } \\
: \text { : }\end{array}$ & \\
\hline 附加信息: & 动积分 & \\
\hline
\end{tabular}

附加信息: 峰被手动积分

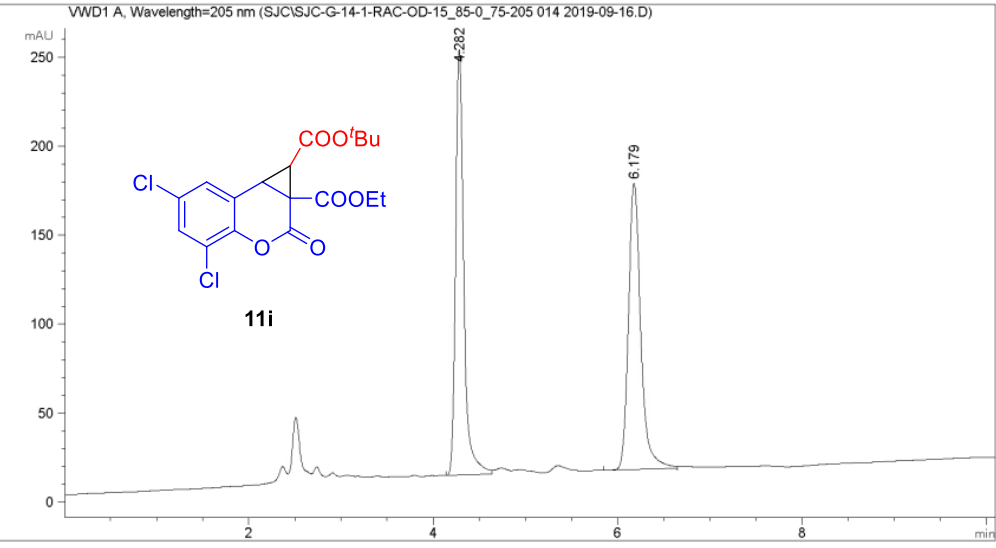

面积百分比报告

$\begin{array}{llll}\text { 排序 } & : & \text { 信号 } & \\ \text { 乘积因子 } & \vdots & 1.0000 \\ \text { 秝释因子 } & : & 1.0000\end{array}$

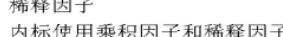

信号 1: VWD1 A, wavelength=205 nm

峰保留时间类型峰宽峰面积峰高峰面积

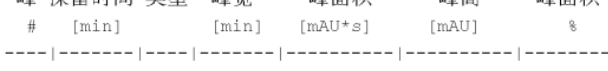

$\begin{array}{llllll}1 & 4.282 \mathrm{BV} & 0.0942 & 1479.36646 & 239.08223 & 49.8271 \\ 2 & 6.179 \mathrm{BV} & 0.1410 & 1489.63599 & 160.95488 & 50.1729\end{array}$

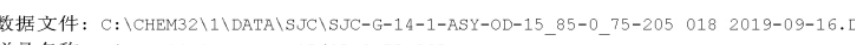
样品名称, $3 j 0-9-14-1-205-00-15 / 85-0.75-205$

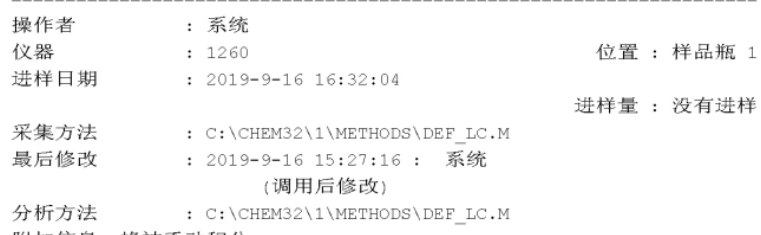

分析方法 $:$ : : \CHEM32\1 1 METHODS $\backslash$ DEF_LC.

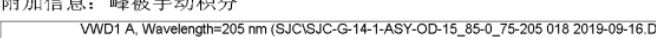

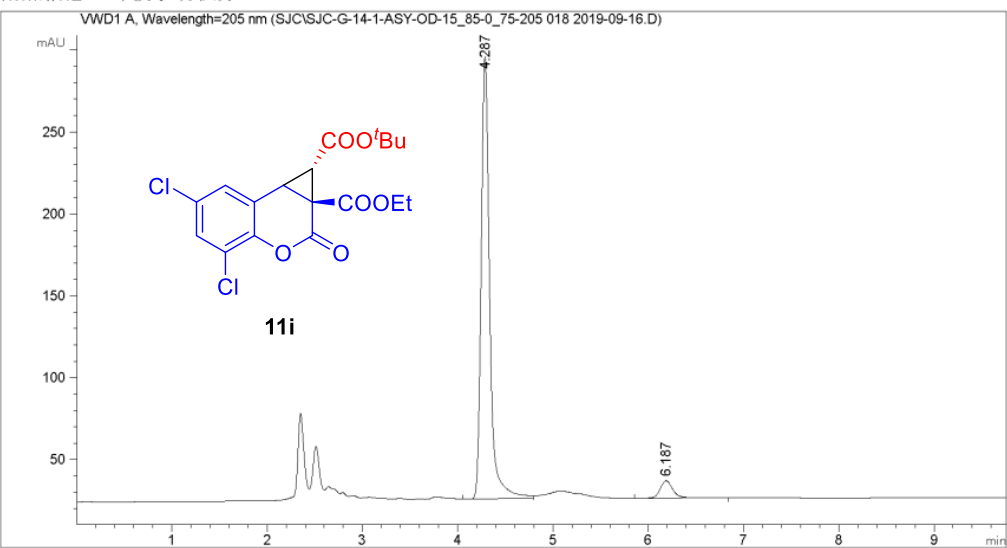

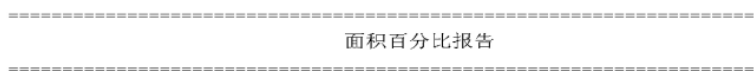

排序

信号

乘积因子

希秚因子

用乘积因子和称释因子

信号 1: VWD1 A, wavelength=205 num

峰保留时间类型峰宽峰面积峰高峰面积

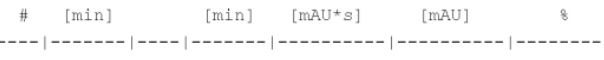

$\begin{array}{lllllll}1 & 4.287 \mathrm{BV} & 0.0946 & 1678.55579 & 269.90268 & 94.5017 \\ 2 & 6.187 & \mathrm{BB} & 0.1394 & 97.65236 & 10.60891 & 5.4983\end{array}$

1260 2019-9-16 16:50:18 系统 


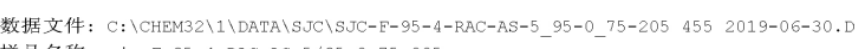

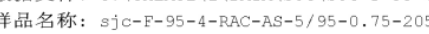

\begin{tabular}{|c|c|c|}
\hline 操作者 & : 系统 & \\
\hline 仪器 & $: 1260$ & 位置：样品瓶 1 \\
\hline 进样日期 & $: 2019-6-30 \quad 10: 29: 02$ & 进样量：没有进样 \\
\hline $\begin{array}{l}\text { 采集方法 } \\
\text { 取后修改 }\end{array}$ & $\begin{array}{c}\text { : C: \CHEM32\1 } 1 \text { METHODS \DEF LC.M } \\
: \text { 2019-6-30 9:29:25: 系统 } \\
\text { (调用后修改) }\end{array}$ & \\
\hline 分析方法 & $\begin{array}{l}C: \backslash C H E M 32 \backslash 1 \backslash M E T H O D S \backslash D E F \text { _LC.M } \\
\text { 办积分 }\end{array}$ & \\
\hline
\end{tabular}

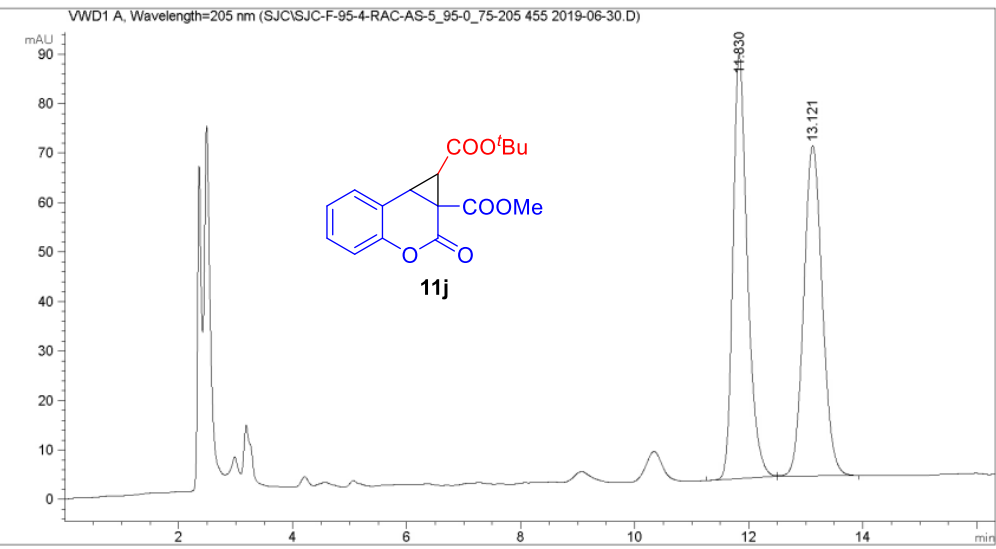

面积百分比报告

$\begin{array}{llll}\text { 排序 } & : & \text { 信号 } & \\ \text { 乘积因子 } & \vdots & 1.0000 \\ \text { 饰䉽因子 } & : & 1.0000\end{array}$

内标使用乘积因子和䊩释因子

信号 1: VWD1 A, wavelength=205 nm

峰保留时间类型峰宽峰面积峰高峰面积

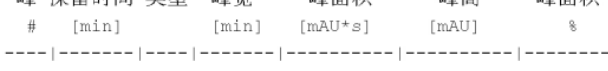

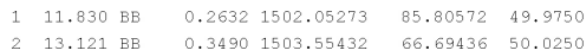

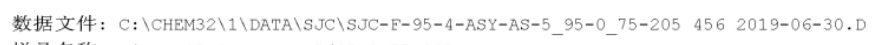

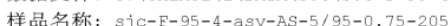

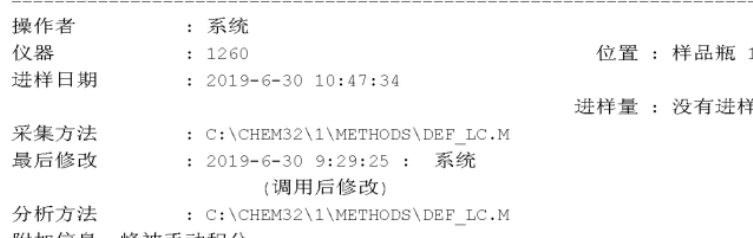

分析方法 : C: \CHEM32\1\METHODS \DEE_LC.

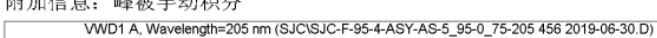

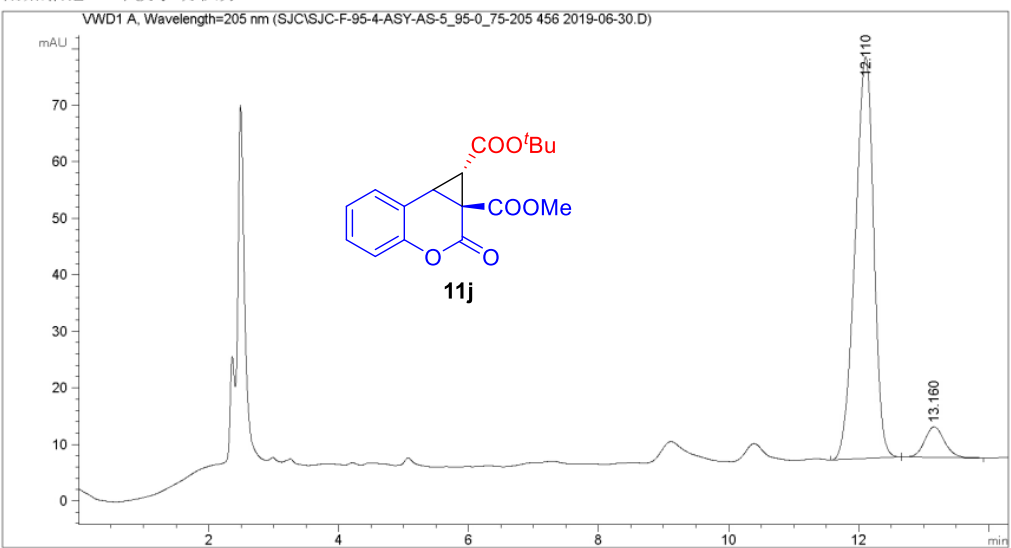

面积百分比报告

排序

乘积因子

它官

稀释因子

1.0000

信号 1: VWD1 A, wavelength=205 nm

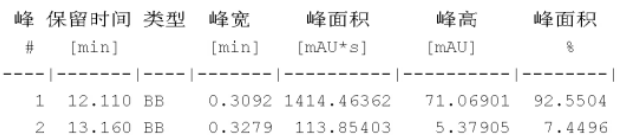




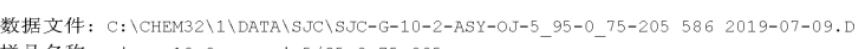
羊品名称: sjc- 9 -10-2-asy-oj-5/95-0.75-20

\begin{tabular}{|c|c|c|}
\hline 操作者 & : 系统 & \\
\hline $\begin{array}{l}\text { 仪器 } \\
\text { 进样日期 }\end{array}$ & $: \quad 1260$ & 位置：样品瓶 1 \\
\hline 进样日期 & $: 2019-7-9 \quad 21: 36: 23$ & 进样量：没有进样 \\
\hline $\begin{array}{l}\text { 采集方法 } \\
\text { 最后修改 }\end{array}$ & $\begin{array}{c}: \text { : C: \CHEM } 2 \backslash 1 \text { 1METHODS \DEF LC.M } \\
: \text { 2019-7-9 19:44:02: 系统 } \\
\text { (调用后修改) }\end{array}$ & \\
\hline 折方法 & $\begin{array}{l}\text { : C: \CHEM32\1 } \backslash \text { METHODS } \backslash D E E_{-} \text {LC.M.M } \\
\text { 动积分 }\end{array}$ & \\
\hline
\end{tabular}

体信息: 峰被手动积分

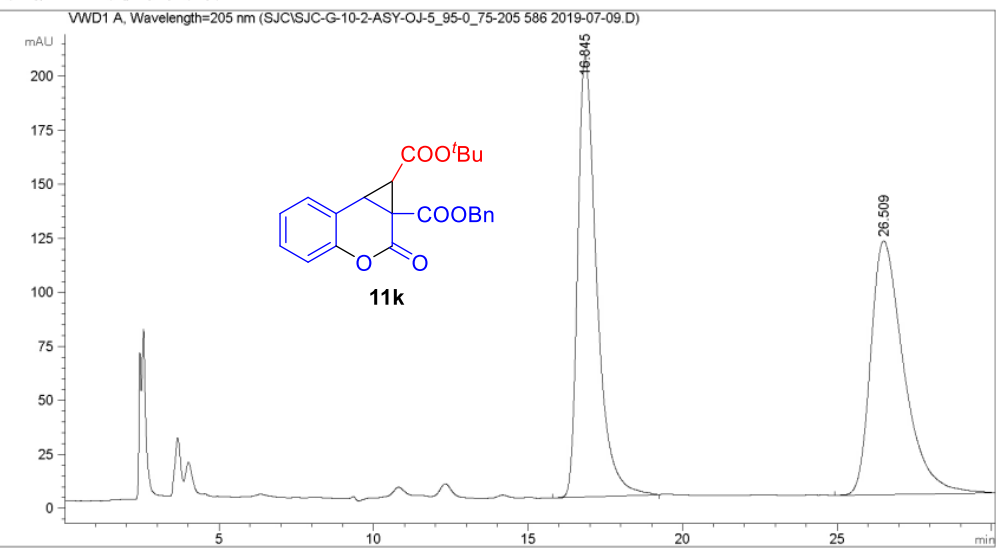

面积百分比报告

$\begin{array}{llll}\text { 排序 } & : & \text { 信号 } & \\ \text { 乘积因子 } & \vdots & 1.0000 \\ \text { 秝释因子 } & : & 1.0000\end{array}$

内标值用乘积因子和残释因

信号 1: VWD1 A, Wavelength=205 nm

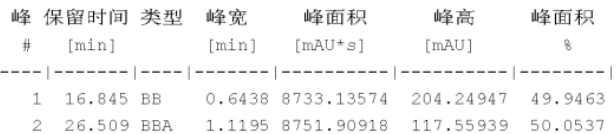

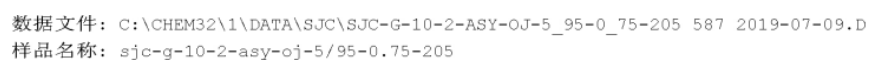

\begin{tabular}{|c|c|c|}
\hline 操作者 & : 系统 & \\
\hline 仪器 & $: 1260$ & 位置：样品瓶 1 \\
\hline 进样日期 & $: 2019-7-9 \quad 22: 07: 37$ & \\
\hline 采集方法 & 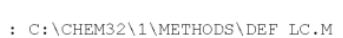 & \\
\hline 最后修改 & $\begin{array}{c}: 2019-7-919: 44: 02: \text { 系统 } \\
\text { (调用后修改) }\end{array}$ & \\
\hline $\begin{array}{l}\text { 分析方法 } \\
\text { 倠加棓 }\end{array}$ & $\begin{array}{l}\text { : C: } \backslash \text { CHEM32\1 \METHODS } \backslash D E E_{-} \text {LC.M } \\
\text { 动积分 }\end{array}$ & \\
\hline
\end{tabular}

倠加信息：峰被手动积分

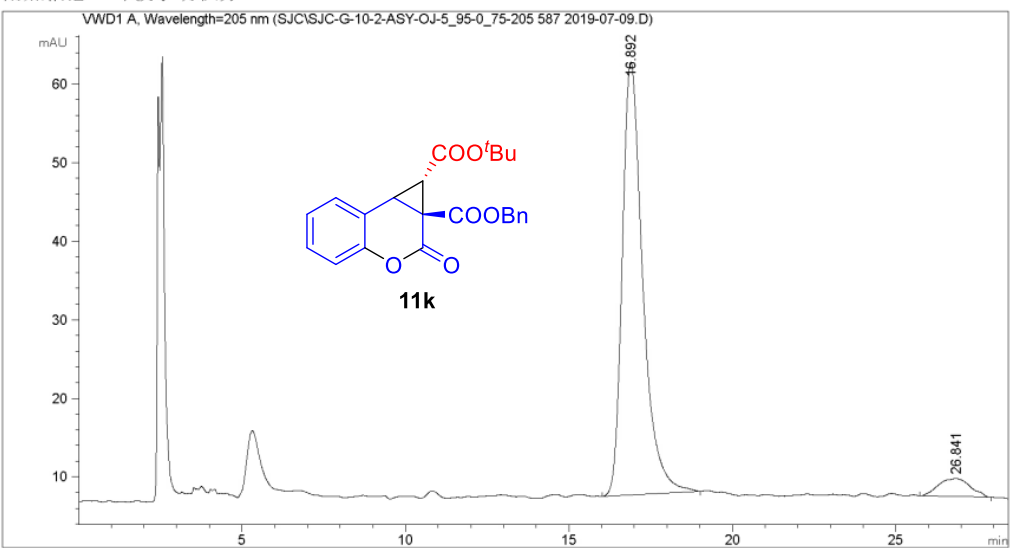

面积百分比报告

排序

乘积因子

位它

稀释因子

: 1.0000

我和稀程因子

信号 1: VWD1 A, wavelength=205 nm

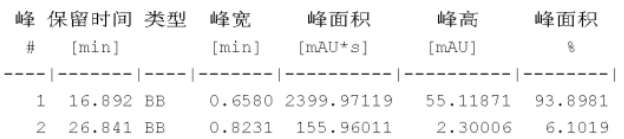

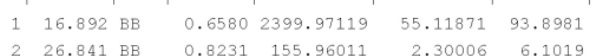

1260 2019-7-10 9:37:14 系统 
数据文件: C: \CHEM32\1\DATA \SJC \SJC-G-34-1-RAC-10_90-0_75-OJ-205862 2019-08-21.D 羊品名称: $s j \mathrm{c}-\mathrm{g}-34-1-\mathrm{RAC}-10 / 90-0.75-\mathrm{OJ}-205$

\begin{tabular}{|c|c|c|}
\hline 操作者 & : 系统 & \\
\hline 仪器 & $: 1260$ & 位置：样品瓶 1 \\
\hline 进样日期 & $: 2019-8-21 \quad 20: 08: 52$ & 进样量：没有进样 \\
\hline $\begin{array}{l}\text { 采集方法 } \\
\text { 取后修改 }\end{array}$ & 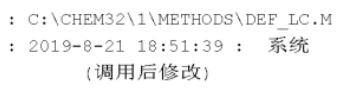 & \\
\hline 折方法 & $\begin{array}{l}C: \backslash C H E M 32 \backslash 1 \backslash M E T H O D S \backslash D E F \text { LC.M } \\
\text { 动积分 }\end{array}$ & \\
\hline
\end{tabular}

附加信息: 峰被手动积分

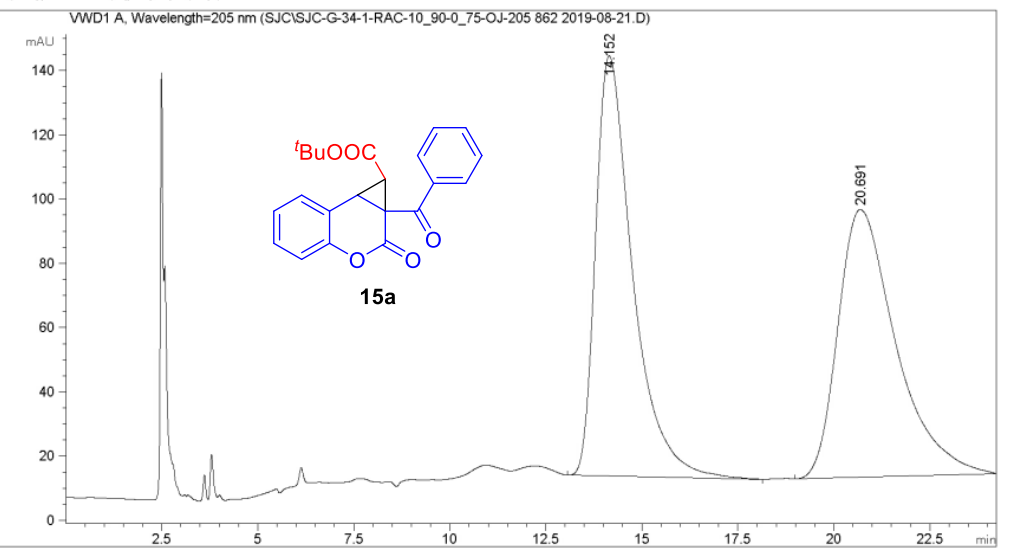

面积百分比报告

$\begin{array}{llll}\text { 排序 } & : & \text { 信号 } & \\ \text { 乘积因子 } & \vdots & 1.0000 \\ \text { 秝释因子 } & : & 1.0000\end{array}$

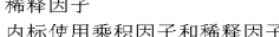

信号 1: VWD1 A, wavelength=205 nm

\section{峰保留时间类型峰宽峰面积峰高峰面积}

\# $[\mathrm{min}][\mathrm{min}][\mathrm{mAU}+s][\mathrm{mAU}] \frac{1}{8}$

$\begin{array}{llllll}1 & 14.152 \text { BB } & 1.0037 & 8785.71387 & 130.78671 & 50.1889\end{array}$

(20)

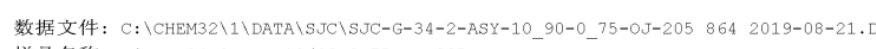
样品名称: s广c- $-3-34-2$-ASY-10/90-0. 75 - OJ-205

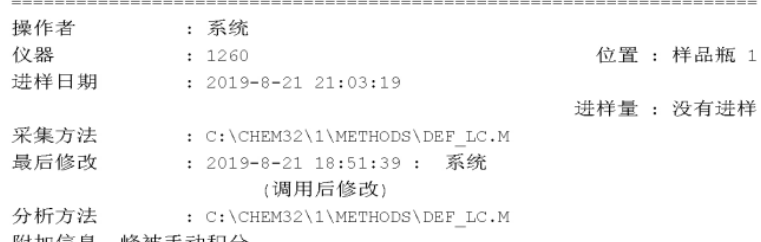

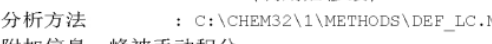

附加信息: 峰被手动积分
WO

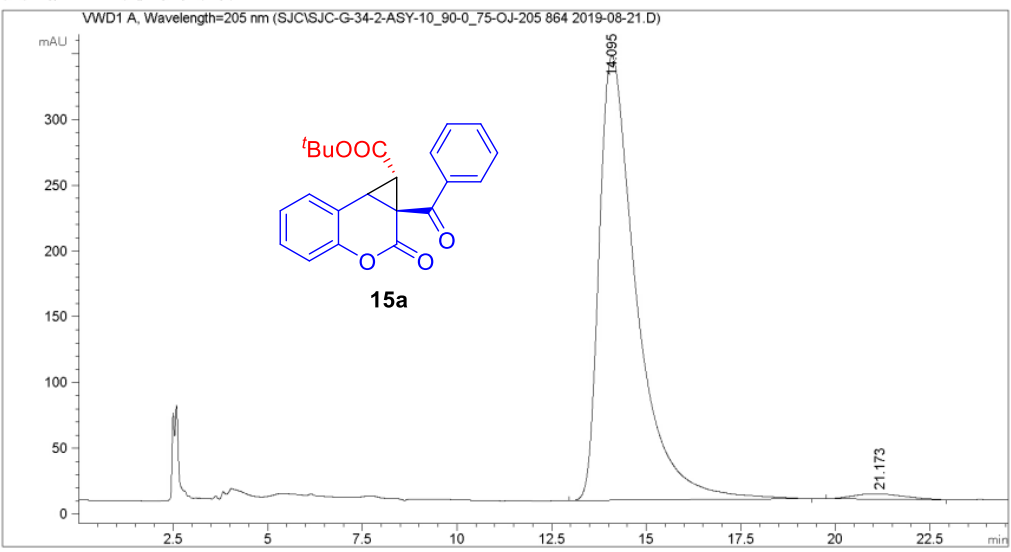

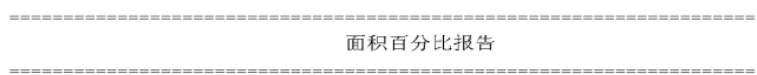

排序

信号

乘积因子

稀释因子

内标使用乘积因子和稼释因子

信号 1: VWD1 A, Wavelength=205 nm

峰保留时间类型峰宽峰面积峰高峰面积

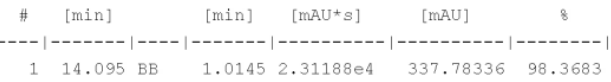

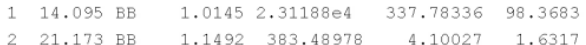

1260 2019-8-21 21:30:28 系统 
数据文件: C: \CHEM32\1\DATA \SJC \SJC-G-38-1-RAC-10_90-0_75-OD-205902 2019-08-24.D 羊品名称: $s j \mathrm{c}-\mathrm{g}-38-1-\mathrm{rac}-10 / 90-0.75-\mathrm{OD}-205$

\begin{tabular}{|c|c|c|}
\hline 操作者 & : 系统 & \\
\hline 仪器 & $: 1260$ & 位置：样品瓶 1 \\
\hline 进样日期 & $: 2019-8-24 \quad 17: 17: 44$ & 进样量：没有进样 \\
\hline $\begin{array}{l}\text { 采集方法 } \\
\text { 最后修改 }\end{array}$ & 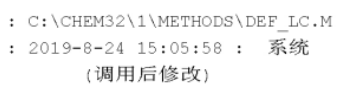 & \\
\hline $\begin{array}{l}\text { 分析方法 } \\
\text { 附加信息: }\end{array}$ & $\begin{array}{l}: \text { : }: \backslash \text { CHEM } 2 \backslash 1 \backslash \text { METHODS } \backslash D E E \text { _LC.M } \\
\text { 三动积分 }\end{array}$ & \\
\hline
\end{tabular}

附加信息: 峰被手动积分

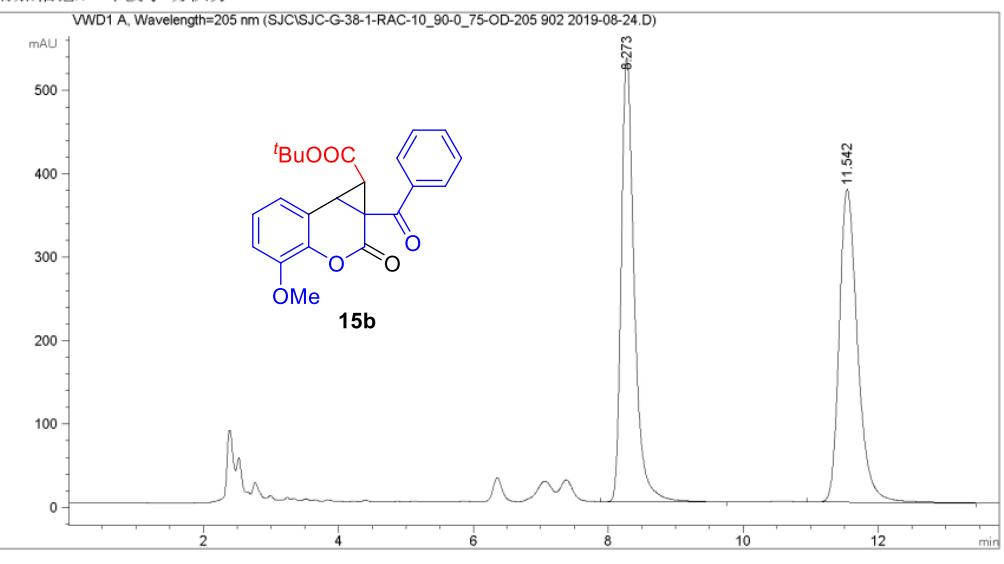

面积百分比报告

$\begin{array}{llll} & \text { 排序 } & \text { 信号 } & \\ \text { 乘积因子 } & \vdots & 1.0000 \\ \text { 稀释因子 } & : & 1.0000\end{array}$

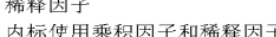

信号 1: VWD1 A, wavelength=205 nm

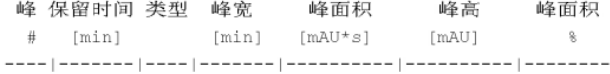

$\begin{array}{llllll}1 & 8.273 \text { BB } & 0.2033 & 7130.17383 & 532.19360 & 50.1919\end{array}$

1260 2019-8-24 17:45:27 系统

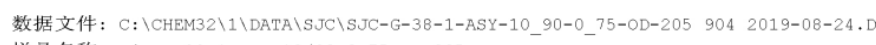
样品名称: sjc- 3 -30-1-asy-10/90-0. $75-0 \mathrm{D}-205$

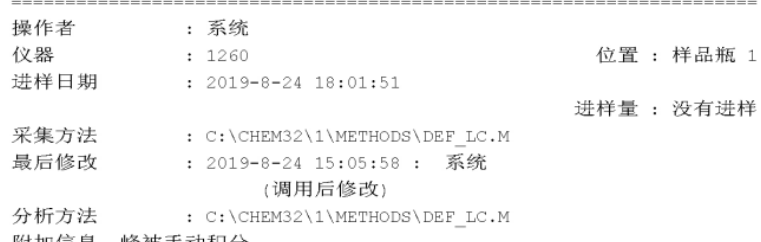

分析方法 $:$ : : \CHEM32\1 1 METHODS $\backslash$ DEF_LC.

的名信息: 峰被手动积分

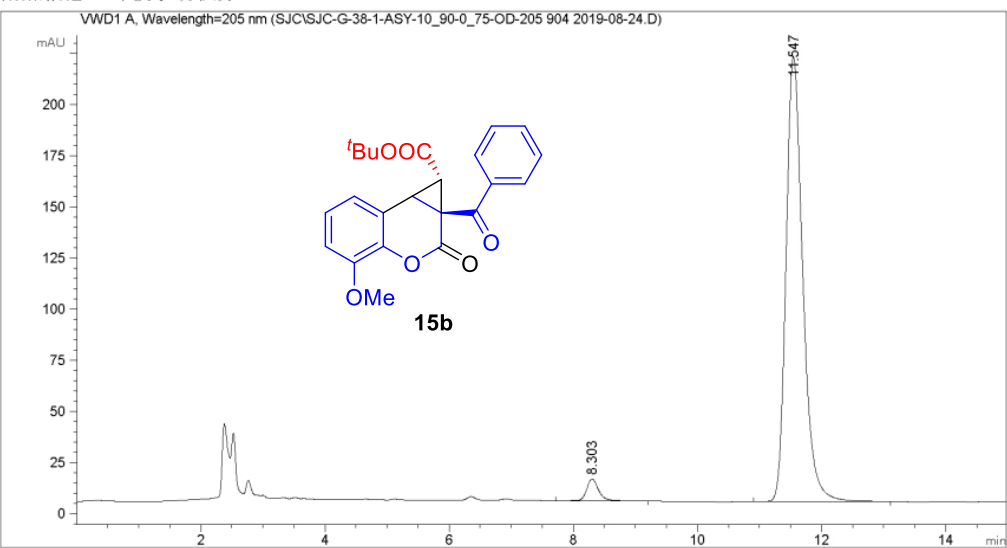

面积百分比报告

排序

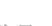

乘积因子

$: \quad 1.0000$

稀释因子

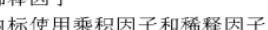

信号 1: VWD1 A, wavelength=205 nm

峰保留时间类型峰䆓峰面积峰高峰面积

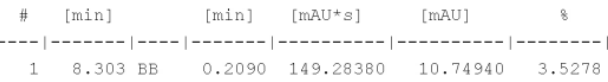

$1260 \quad 2019-8-24 \quad 18: 17: 43$ 系统 


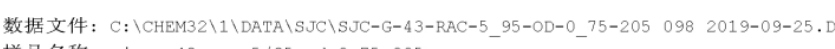

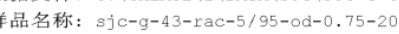

\begin{tabular}{|c|c|c|}
\hline 操作者 & : 系统 & \\
\hline 仪器 & $: 1260$ & 位置：样品瓶 1 \\
\hline 进样日期 & $: 2019-9-25 \quad 20: 08: 27$ & 进样量：没有进样 \\
\hline $\begin{array}{l}\text { 采集方法 } \\
\text { 最后修改 }\end{array}$ & $\begin{array}{l}\text { : C: } \backslash \text { CHEM } M 2 \backslash 1 \backslash \text { METHODS } \backslash \text { DEF_LC.M } \\
: \text { 2019-9-25 19:40:21 : 系统 } \\
\text { (调用后修改) }\end{array}$ & \\
\hline $\begin{array}{l}\text { 分析方法 } \\
\text { 附加信息: }\end{array}$ & $\begin{array}{l}C: \backslash C H E M 32 \backslash 1 \backslash M E T H O D S \backslash D E F \text { LCC.M } \\
\text { 动积分 }\end{array}$ & \\
\hline
\end{tabular}

附加信息: 峰被手动积分

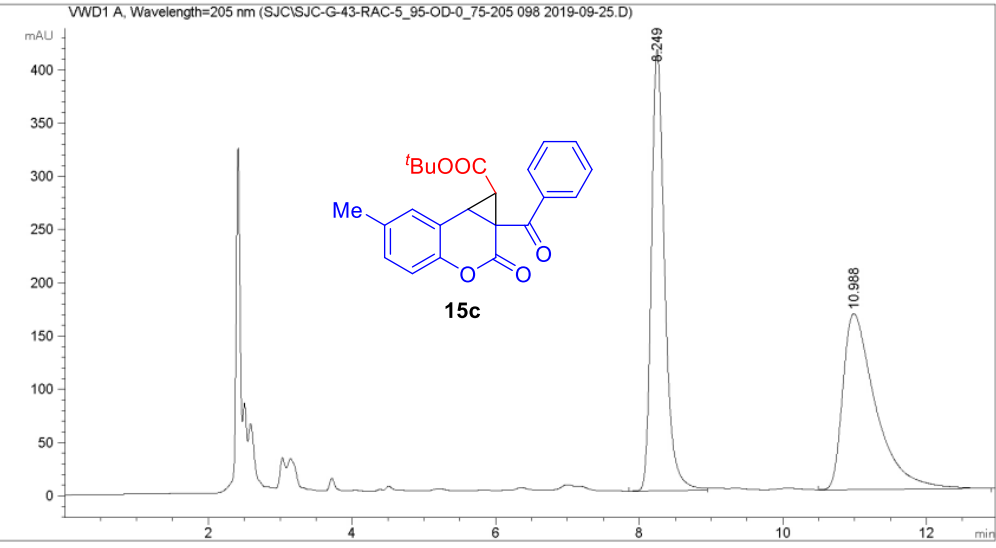

面积百分比报告

$\begin{array}{llll}\text { 排序 } & : & \text { 信号 } & \\ \text { 乘积因子 } & \vdots & 1.0000 \\ \text { 秝释因子 } & : & 1.0000\end{array}$

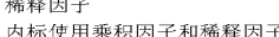

信号 1: VWD1 A, wavelength=205 nm

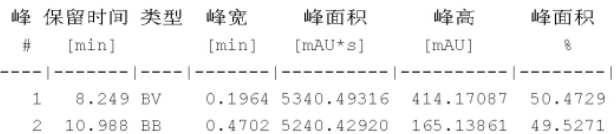

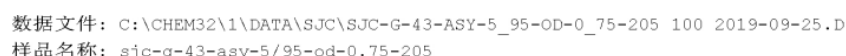

\begin{tabular}{|c|c|c|}
\hline 操作者 & : 系统 & \\
\hline 仪器 & $: 1260$ & 位置：样品瓶 1 \\
\hline 进样日期 & $: 2019-9-25 \quad 20: 35: 57$ & 进样量：没有进样 \\
\hline $\begin{array}{l}\text { 采集方法 } \\
\text { 最后修改 }\end{array}$ & 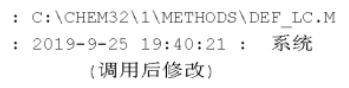 & \\
\hline 分析方法 & 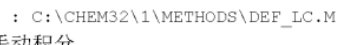 & \\
\hline
\end{tabular}

分析方法 $:$ : : \CHEM32\1 1 METHODS $\backslash$ DEF_LC.

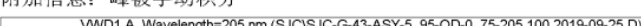

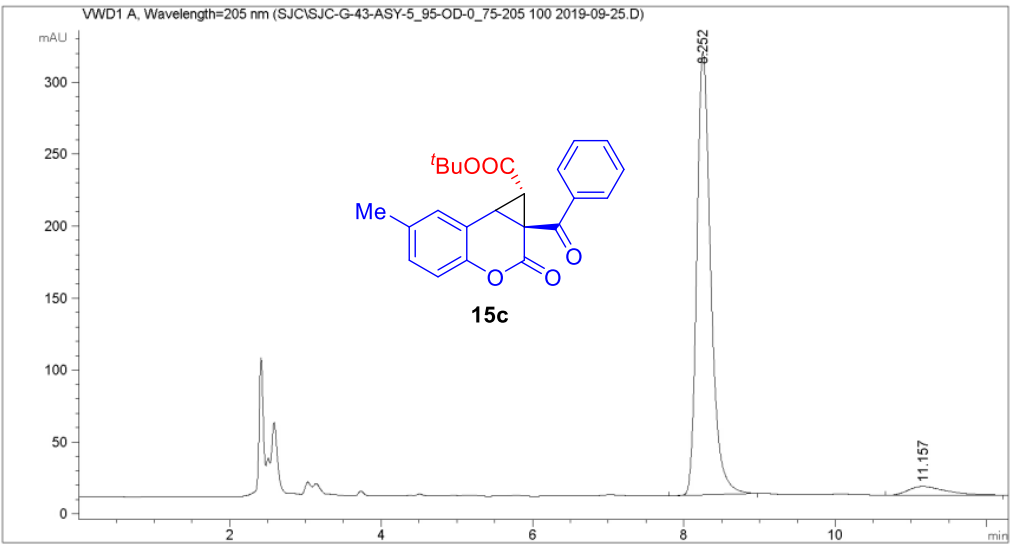

面积百分比报告

排序

信号

乘积因子

稀释因子

和称释因子

信号 1: VWD1 A, wavelength=205 $\mathrm{nm}$

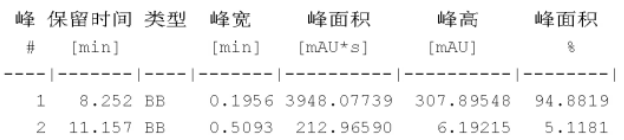

1260 2019-9-25 20:49:07 系统 


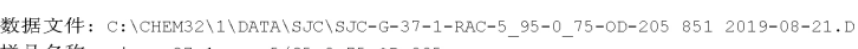
羊品名称: Sic- $9-37-1-20 \mathrm{C}-5 / 95-0.75-0 \mathrm{D}-205$

\begin{tabular}{|c|c|c|}
\hline 操作者 & : 系统 & \\
\hline $\begin{array}{l}\text { 仪器 } \\
\text { 讲样日期 }\end{array}$ & $: \quad 1260$ & 位置：样品瓶 1 \\
\hline 进样日期 & : 2019-8-21 11:06:50 & 进样量：没有进样 \\
\hline $\begin{array}{l}\text { 采集方法 } \\
\text { 最后修改 }\end{array}$ & $\begin{array}{c}: \text { : C: \CHEM } 2 \backslash 1 \text { 1METHODS \DEF_LC.M } \\
: 2019-8-219: 16: 22: \text { 系统 } \\
\text { (调用后修改) }\end{array}$ & \\
\hline 析方法 & $\begin{array}{l}\text { : C: } \backslash \text { CHEM32\1 } \\
\text { 动积分 }\end{array}$ & \\
\hline
\end{tabular}

附加信息: 峰被手动积分

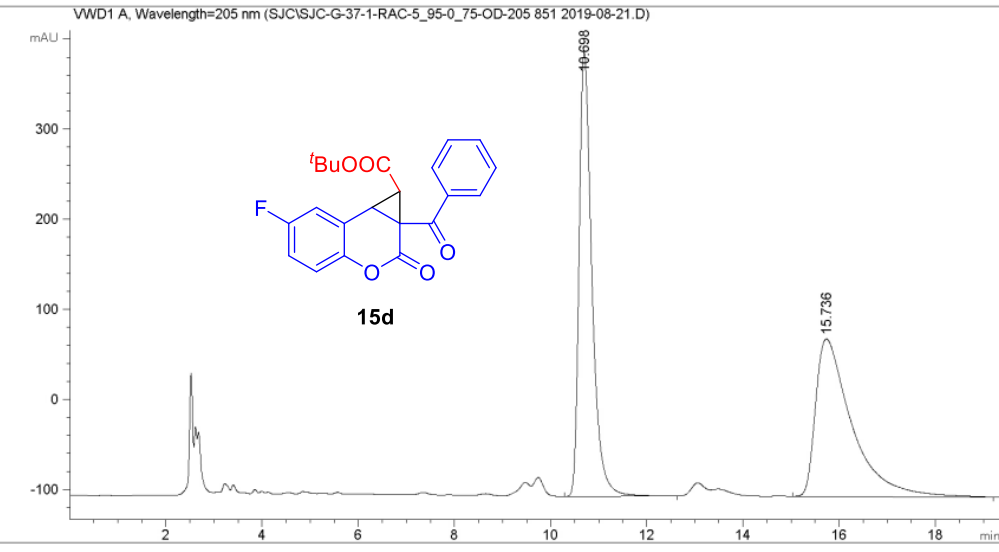

面积百分比报告

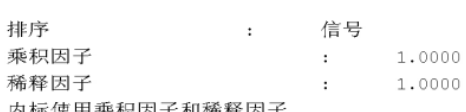

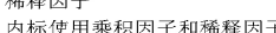

信号 1: VWD1 A, wavelength=205 nm

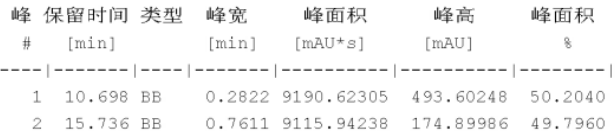

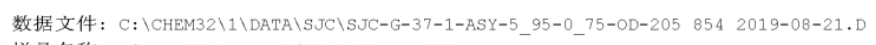

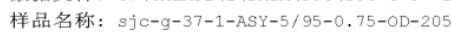

\begin{tabular}{|c|c|c|}
\hline \multirow{3}{*}{$\begin{array}{l}\text { 操作者 } \\
\text { 仪器 } \\
\text { 进样日期 }\end{array}$} & : 系统 & \\
\hline & $\because 1260$ & 位置：样品瓶 1 \\
\hline & $: 2019-8-21 \quad 14: 53: 51$ & \\
\hline 采集方法 & : C: \CHEM32\1\METHODS \DEF LC.M & \\
\hline 最后修改 & $\begin{array}{c}: \text { 2019-8-21 } 14: 16: 37: \text { 系统 } \\
\text { (调用后修改) }\end{array}$ & \\
\hline $\begin{array}{l}\text { 分析方法 } \\
\text { 附加信息: }\end{array}$ & $\begin{array}{l}\text { : C: \CHEM32\1 } \backslash \text { METHODS } \backslash D E E_{-} \text {LC.M } \\
\text { 动积分 }\end{array}$ & \\
\hline
\end{tabular}

倠加信息: 峰被手动积分

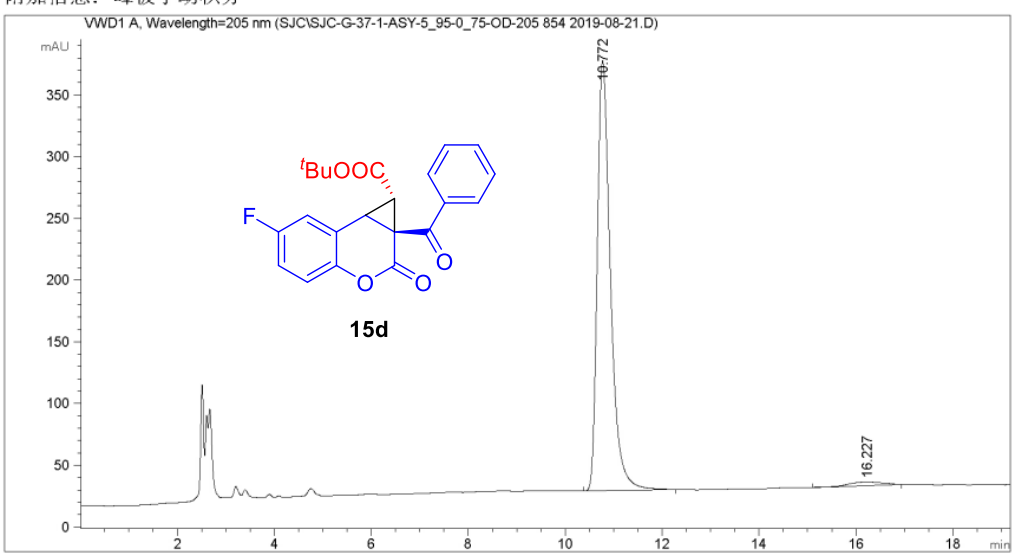

面积百分比报告

排序

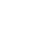

乘积因子

稀释因子 : 1.0000

和稀释因子

信号 1: VWD1 A, wavelength=205 num

峰 保留时间 类型峰宽峰面积峰高峰面积

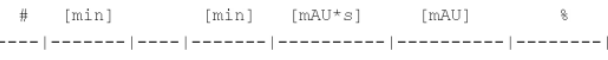

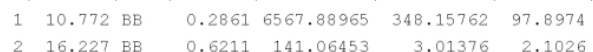

1260 2019-8-21 16:53:05 系统 
数据文件: C: \CHEM32\1\DATA \SJC \SJC-G-37-2-RAC-10_90-0_75-OD-205895 2019-08-24.D 样品名称: $3 j \mathrm{C}-9-37-2-\mathrm{RAC}-10 / 90-0.75-\mathrm{OD}-205$

\begin{tabular}{|c|c|c|}
\hline \multirow{3}{*}{$\begin{array}{l}\text { 操作者 } \\
\text { 仪器 } \\
\text { 进样日期 }\end{array}$} & : 系统 & \\
\hline & $: 1260$ & 位置：样品瓶 \\
\hline & : 2019-8-24 15:24:56 & 进样量：没有进样 \\
\hline 采集方法 & 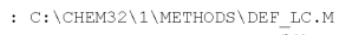 & \\
\hline 最后修改 & $\begin{array}{c}\text { : 2019-8-24 15:05:58： 系统 } \\
\text { (调用后修改) }\end{array}$ & \\
\hline 法 & 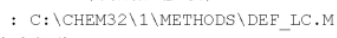 & \\
\hline
\end{tabular}

附加信息: 峰被手动积分

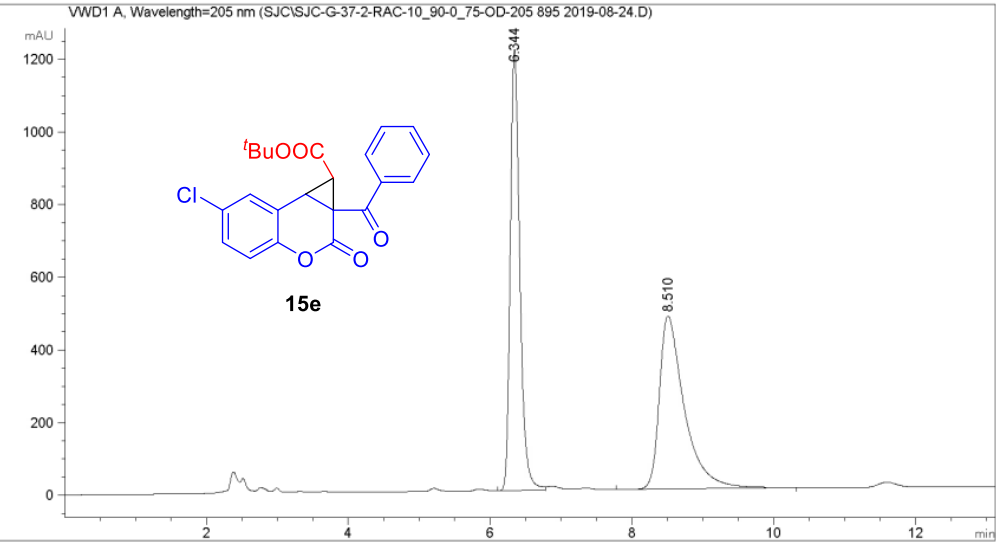

面积百分比报告

$\begin{array}{llll}\text { 排序 } & : & \text { 信号 } & \\ \text { 乘积因子 } & \vdots & 1.0000 \\ \text { 秝释因子 } & : & 1.0000\end{array}$

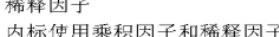

信号 1: VWD1 A, wavelength=205 nm

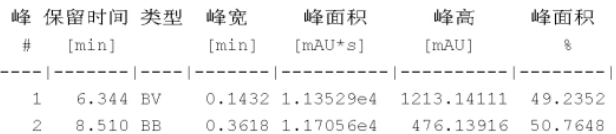

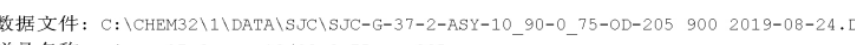
样品名称: $050-37-37-2-39 y-10 / 90-0.75-0 D-205$

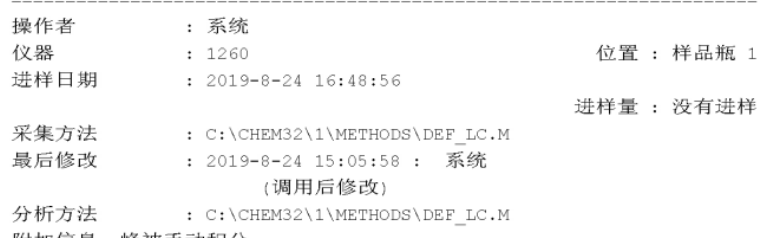

分析方法 : : : \CHEM32\1\METHODS \DEF_LC.

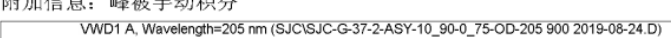

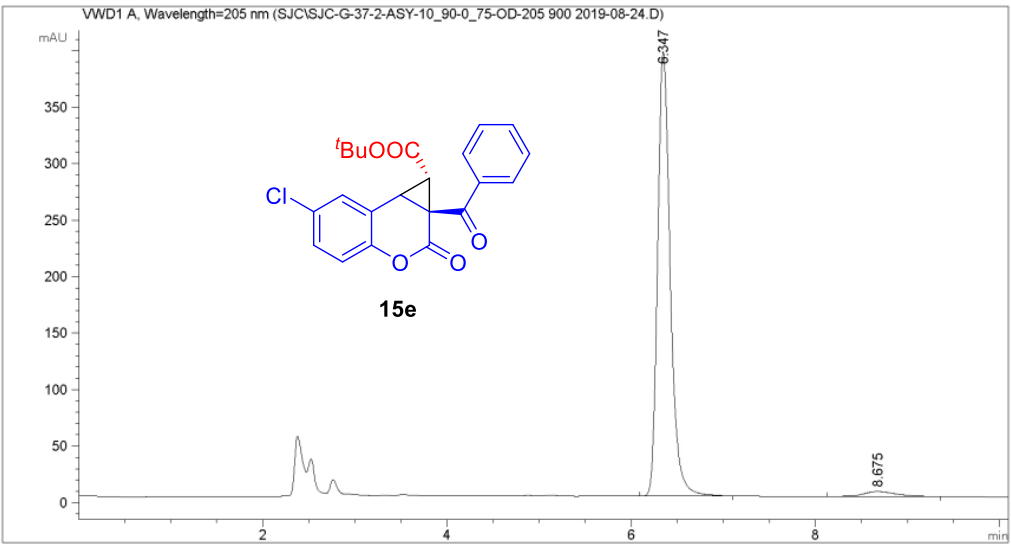

面积百分比报告

排序

乘积因子

言号

脪释因于

$: \quad 1.0000$

我和和称释因子

信号 1: VWD1 A, wavelength=205 nm

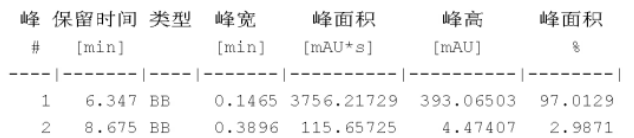

1260 2019-8-24 17:01:14 系统 


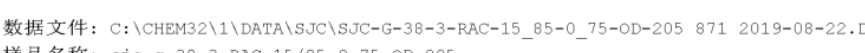
羊品名称: $s j \mathrm{C}-\mathrm{g}-38-3-\mathrm{RAC}-15 / 85-0.75-\mathrm{OD}-205$

\begin{tabular}{|c|c|c|}
\hline 操作者 & : 系统 & \\
\hline 仪器 & $: 1260$ & 位置：样品瓶 1 \\
\hline 进样日期 & $: 2019-8-22 \quad 19: 15: 38$ & 进样量：没有进样 \\
\hline $\begin{array}{l}\text { 采集方法 } \\
\text { 取后修改 }\end{array}$ & 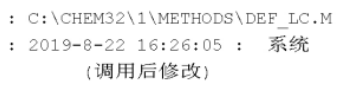 & \\
\hline $\begin{array}{l}\text { 分析方法 } \\
\text { 附加信息: }\end{array}$ & 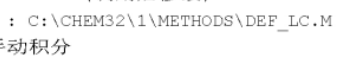 & \\
\hline
\end{tabular}

附加信息：峰被手动积分

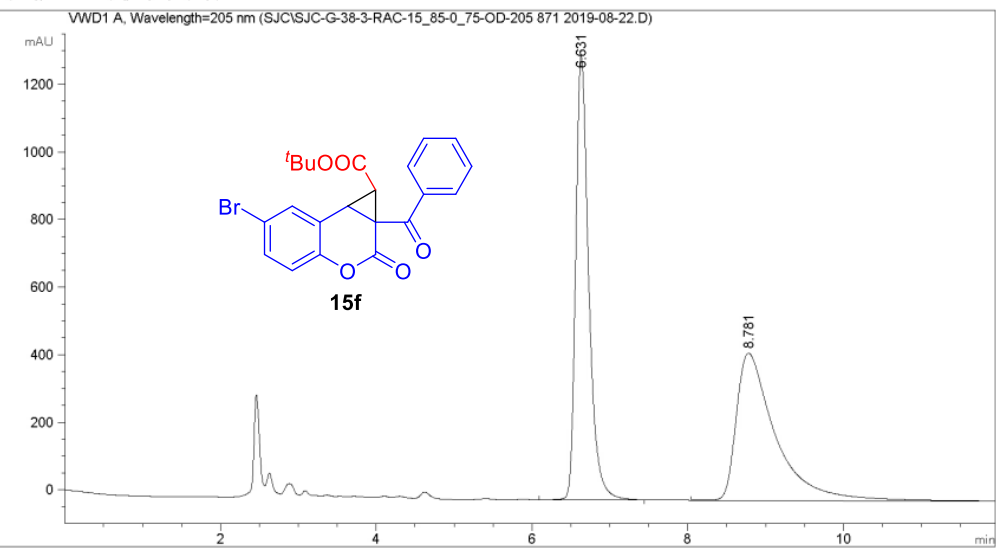

面积百分比报告

$\begin{array}{llll}\text { 排序 } & : & \text { 信号 } & \\ \text { 乘积因子 } & \vdots & 1.0000 \\ \text { 秝释因子 } & : & 1.0000\end{array}$

内标使用乘积因子和顽船因子

信号 1: VWD1 A, wavelength=205 nm

\section{峰保留时间类型峰宽峰面积峰高峰面积}

\# $[\mathrm{min}] \quad[\mathrm{min}][\mathrm{mAU}+s][\mathrm{mAU}] \frac{1}{8}$

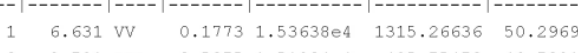

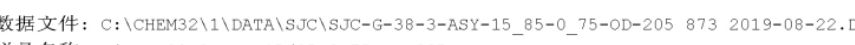

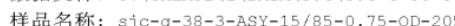

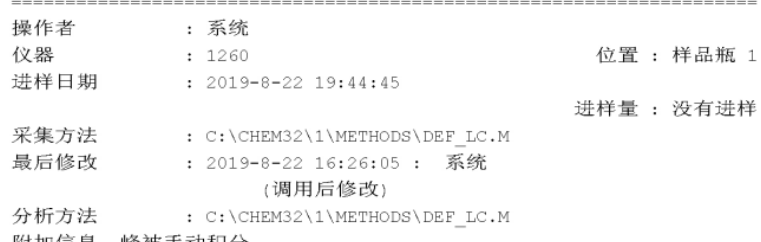

分析方法 $:$ : : \CHEM32\1 1 METHODS $\backslash$ DEF_LC.

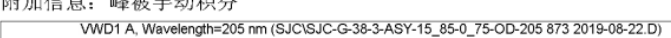

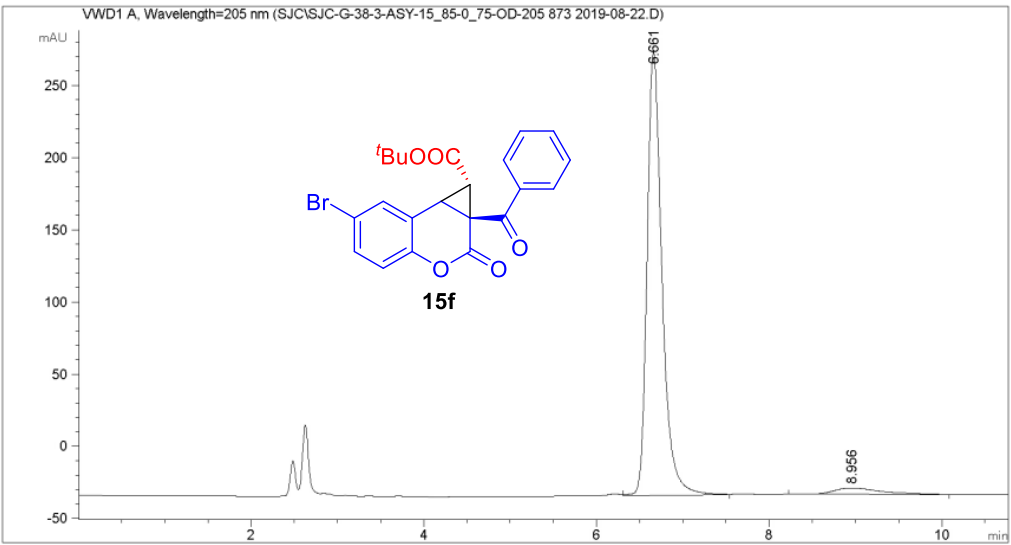

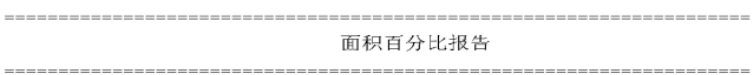

排序

信号

乘积因子

稀秚因子

示使用乘积因子和稀释因子

信号 1: VWD1 A, wavelength=205 nm

峰 保留时间 类型峰宽峰面积峰高峰面积

\# $[\mathrm{min}]$

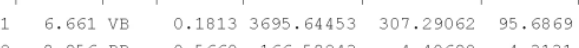

2019-0-28 $15: 28: 35$ 系纸

页 $1 / 2$ 


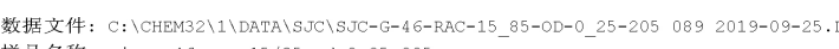
羊品名称: sjc- $9-46-r a c-15 / 85-0 d-0.25-205$

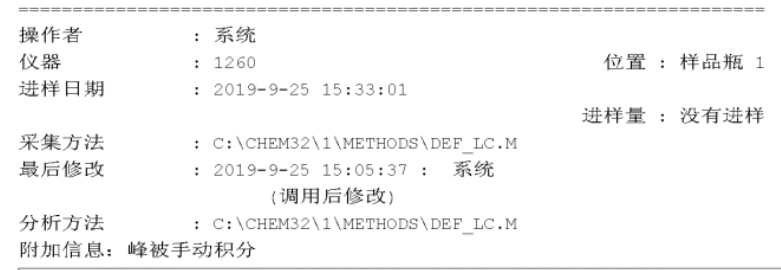

附加信息: 峰被手动积分

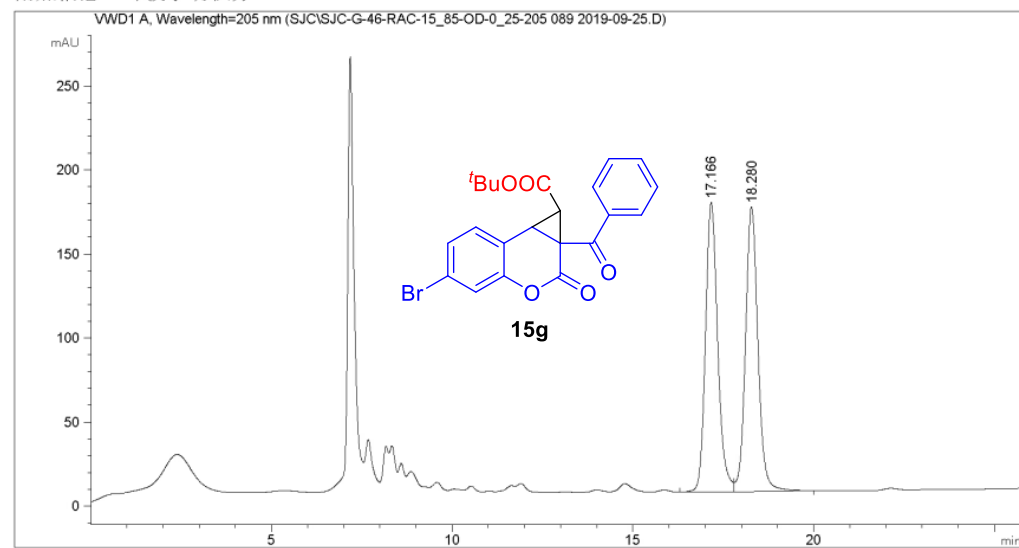

面积百分比报告

$\begin{array}{llll}\text { 排序 } & : & \text { 信号 } & \\ \text { 乘积因子 } & \vdots & 1.0000 \\ \text { 秝释因子 } & : & 1.0000\end{array}$

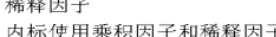

信号 1: VWD1 A, wavelength=205 nm

峰保留时间类型峰宽峰面积峰高峰面积

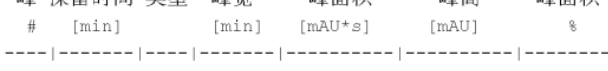

$\begin{array}{llllll}1 & 17.166 \text { BV } & 0.3602 & 4043.44360 & 172.06198 & 49.9566\end{array}$

1260 2019-9-25 16:47:05 系统

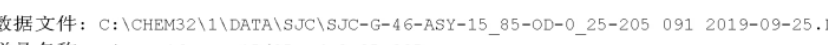

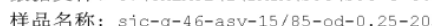

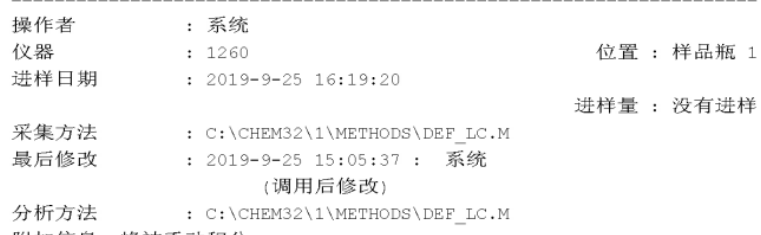

分析方法 $:$ : : \CHEM32\1 1 METHODS $\backslash$ DEF_LC.

附加信息: 峰被手动积分

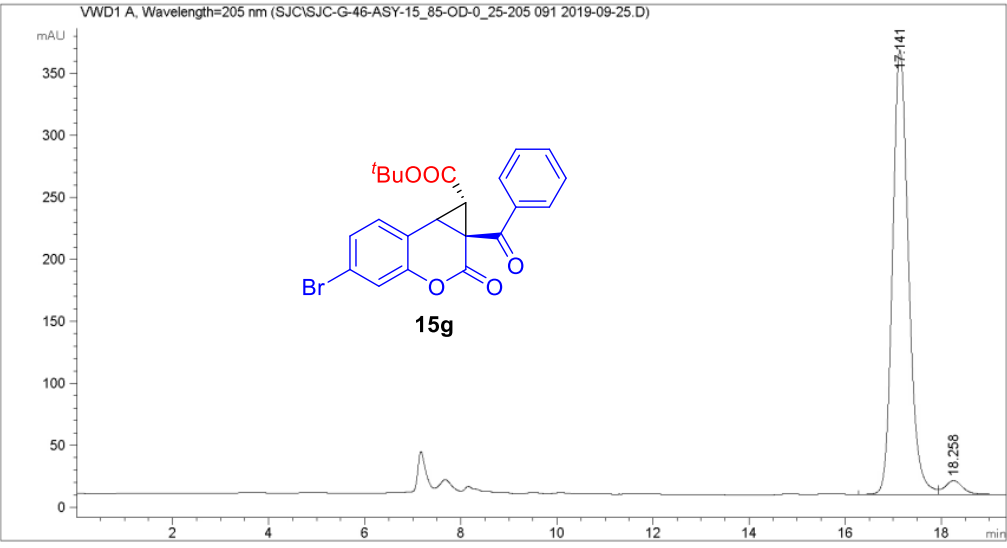

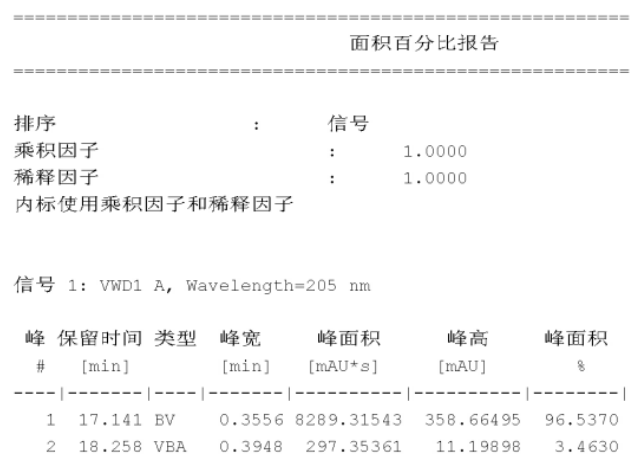

1260 2019-9-25 16:46:46 系统 


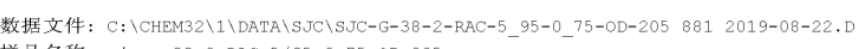
羊品名称: sjc-g-38-2-RAC-5/95-0.75-OD-20

\begin{tabular}{|c|c|c|}
\hline 操作者 & : 系统 & \\
\hline 仪器 & $: 1260$ & 位置：样品瓶 1 \\
\hline 进样日期 & $: 2019-8-22 \quad 22: 39: 25$ & 进样量：没有进样 \\
\hline 采集方法后修改 & 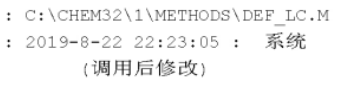 & \\
\hline 分析方法 & 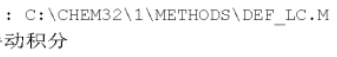 & \\
\hline
\end{tabular}

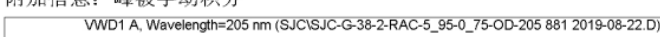

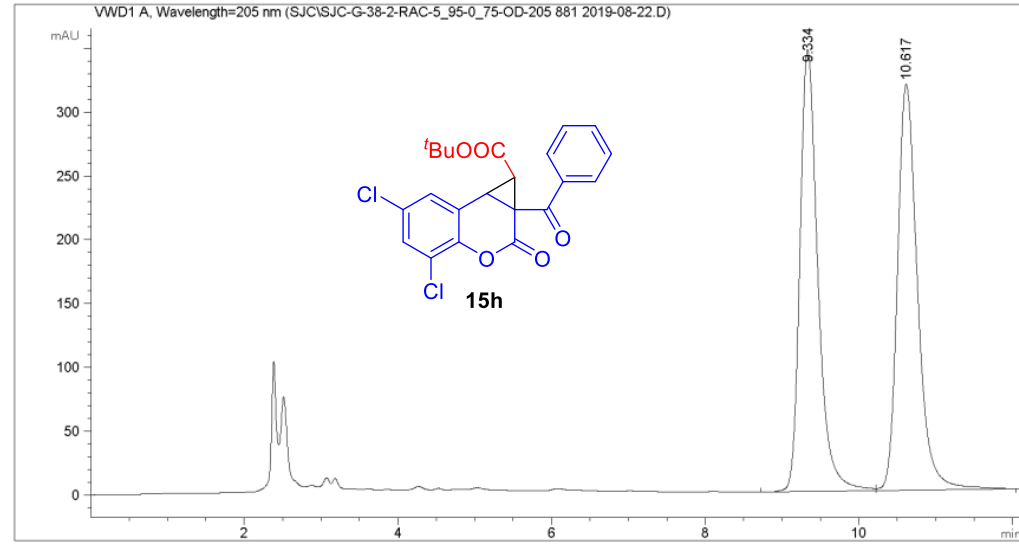

面积百分比报告

$\begin{array}{llll}\text { 排序 } & : & \text { 信号 } & \\ \text { 乘积因子 } & \vdots & 1.0000 \\ \text { 饰䉽因子 } & : & 1.0000\end{array}$

内标保因乘积因子和稀释因子

信号 1: VWD1 A, wavelength=205 nm

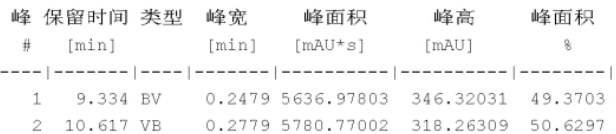

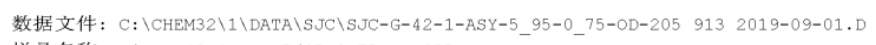
样品名称: sic- $9-42-1-a s y-5 / 95-0$. 75-0D-205

\begin{tabular}{|c|c|c|}
\hline \multirow{3}{*}{$\begin{array}{l}\text { 操作者 } \\
\text { 仪器 } \\
\text { 进样日期 }\end{array}$} & : 系统 & \\
\hline & $\because 1260$ & 位置：样品瓶 1 \\
\hline & : 2019-9-1 9:56:51 & \\
\hline 采集方法 & : C: \CHEM32\1\METHODS \DEF LC.M & \\
\hline 最后修改 & $\begin{array}{c}: \text { :2019-9-1 9:16:00： 系统 } \\
\text { (调用后修改) }\end{array}$ & \\
\hline 分析方法 & $\begin{array}{l}: C: \backslash C H E M 32 \backslash 1 \backslash M E T H O D S \backslash D E E_{-} \text {LC.M } \\
\text { =动积分 }\end{array}$ & \\
\hline
\end{tabular}

附加信息: 峰被手动积分
WW

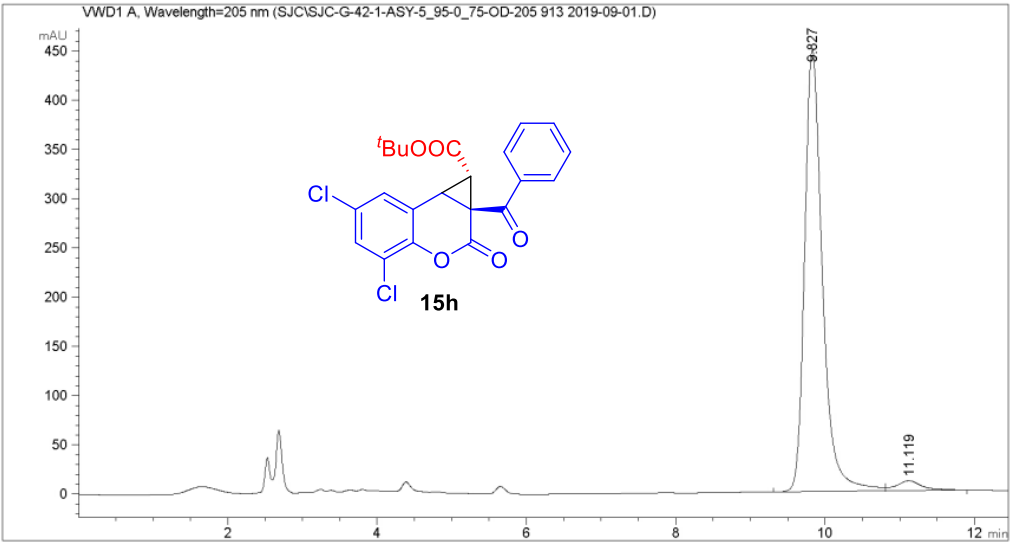

面积百分比报告

排序

乘积因子

它官

脪释因于

1.0000

内标使乘积因子和稀释因子

信号 1: VWD1 A, wavelength=205 nm

峰保留时间类型峰䆓峰面积峰高峰面积

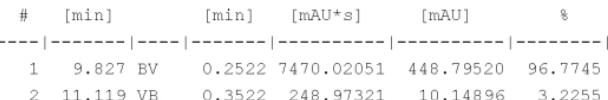

1260 2019-9-2 15:41:44 系统 


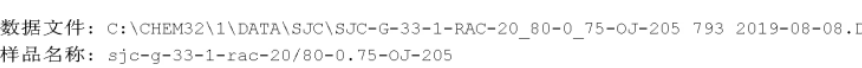

\begin{tabular}{|c|c|c|}
\hline 操作者 & : 系统 & \\
\hline 仪器 & $: 1260$ & 位置：样品瓶 1 \\
\hline 进样日期 & $: 2019-8-8 \quad 20: 01: 40$ & 进样量：没有进样 \\
\hline 采集方法 & $\begin{array}{c}: \text { : C: \CHEM } 32 \backslash 1 \mid \text { METHODS } \backslash \text { DEF LC.M } \\
: \text { 2019-8-8 19:08: }: 88 \text { : 系统 } \\
\text { (调用后修改) }\end{array}$ & \\
\hline $\begin{array}{l}\text { 分析方法 } \\
\text { 附加信息: }\end{array}$ & $\begin{array}{l}\text { : C: } \backslash \text { CHEM32\1 \METHODS } \backslash \text { DEF_LC.M } \\
\text { 动积分 }\end{array}$ & \\
\hline
\end{tabular}

附加信息：峰被手动积分

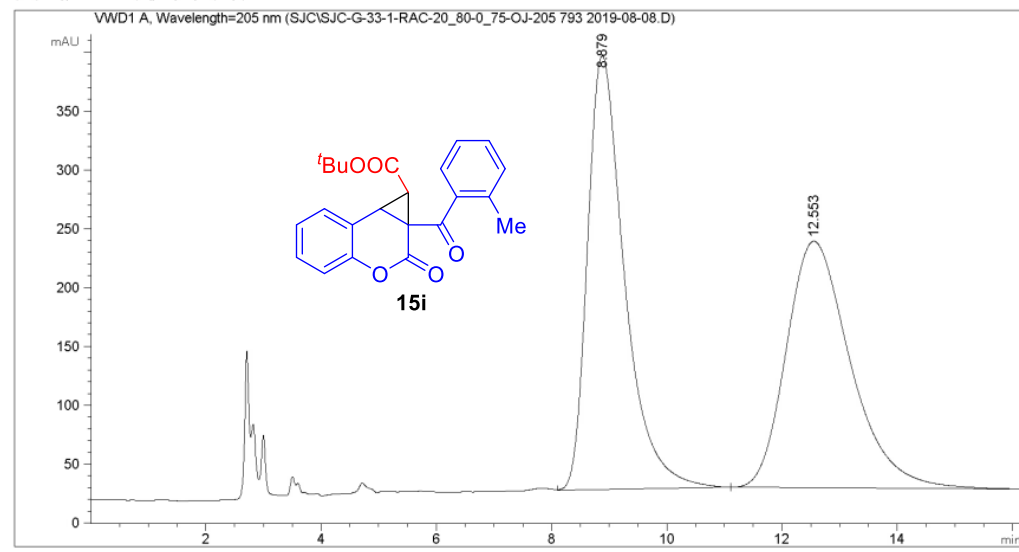

面积百分比报告

$\begin{array}{llll}\text { 排序 } & : & \text { 信号 } & \\ \text { 乘积因子 } & \vdots & 1.0000 \\ \text { 秝释因子 } & : & 1.0000\end{array}$

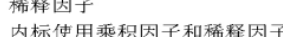

信号 1: VWD1 A, wavelength=205 nm

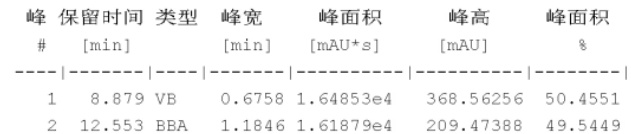

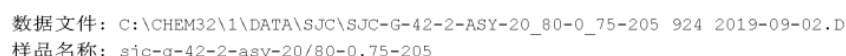

\begin{tabular}{|c|c|c|}
\hline 操作者 & : 素统 & \\
\hline 仪器 & $: 1260$ & 位置：样品瓶 1 \\
\hline 进样日期 & $:$ :2019-9-2 15:15:40 & 没有讲新 \\
\hline 采集方法 & $: C: \backslash C H E M 32 \backslash 1 \backslash M E T H O D S \backslash D E F \_L C . M$ & \\
\hline 最后修改 & $\begin{array}{r}\text { : 2019-9-2 14:35:55：系统 } \\
\text { (调用后修改) }\end{array}$ & \\
\hline
\end{tabular}

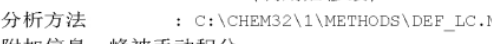

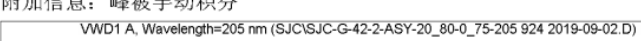

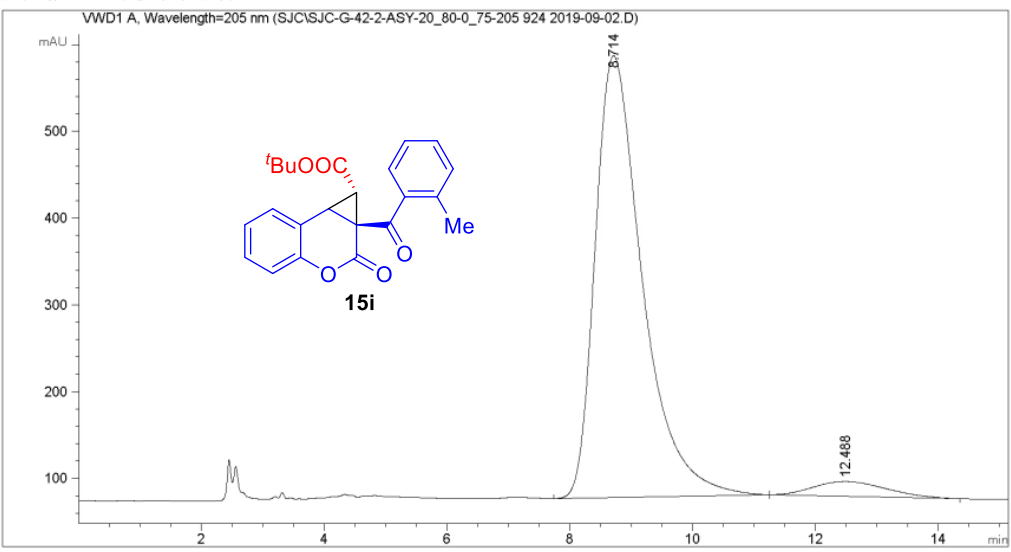

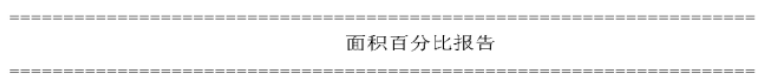

排序

信号

乘积因子

稀释因子

内标使用乘积因子和称释因子

信号 1: VWD1 A, wavelength=205 num

峰保留时间类型峰宽峰面积峰高峰面积

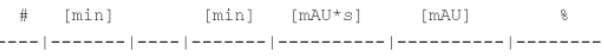

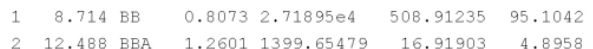

1260 2019-9-2 15:39:59 系统 


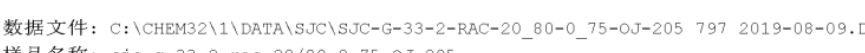
羊品名称: sjc-g-33-2-rac-20/80-0.75-0J-20

\begin{tabular}{|c|c|c|}
\hline 操作者 & : 系统 & \\
\hline 仪器 & $: 1260$ & 位置：样品瓶 1 \\
\hline 进样日期 & $: 2019-8-9$ 10:23:01 & 进样量：没有进样 \\
\hline $\begin{array}{l}\text { 采集方法 } \\
\text { 最后修改 }\end{array}$ & 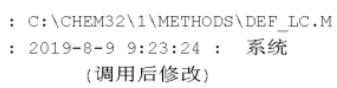 & \\
\hline $\begin{array}{l}\text { 分析方法 } \\
\text { 附加信息: }\end{array}$ & $\begin{array}{l}: \text { : }: \backslash \text { CHEM } 2 \backslash 1 \backslash \text { METHODS } \backslash D E E \text { _LC.M } \\
\text { 三动积分 }\end{array}$ & \\
\hline
\end{tabular}
陌前信息: 峰被手动积分

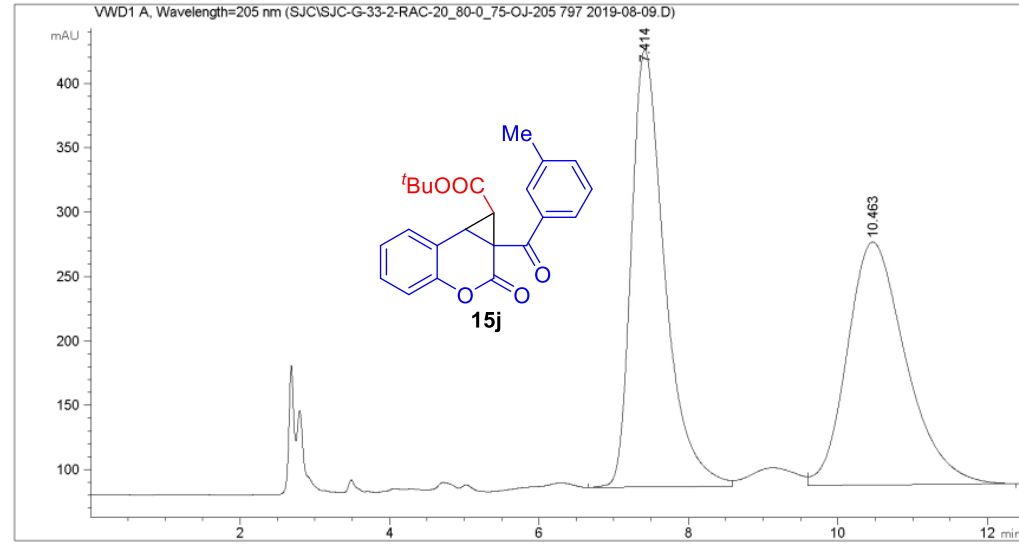

面积百分比报告

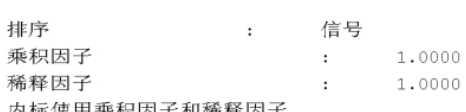

内标值用乘和因子和㱠船因子

信号 1: VWD1 A, wavelength=205 nm

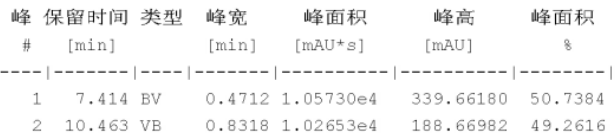

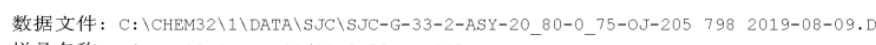
样品名称: $5 j c-9-33-2-29 y-20 / 80-0.75-0.5-205$

\begin{tabular}{|c|c|c|}
\hline 操作者 & : 素统 & \\
\hline 仪器 & $: 1260$ & 位置：样品瓶 1 \\
\hline 进样日期 & $: 2019-8-9 \quad 10: 38: 18$ & 没有讲新 \\
\hline 采集方法 & $: C: \backslash C H E M 32 \backslash 1 \backslash M E T H O D S \backslash D E F \_L C . M$ & \\
\hline 最后修改 & $\begin{array}{c}: \text { 2019-8-9 9:23:24： 系统 } \\
\text { (调用后修改) }\end{array}$ & \\
\hline
\end{tabular}

分析方法 : C: \CHEM32\1 \METHODS \DEF_LC.11

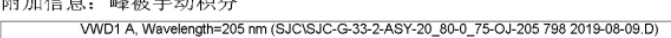

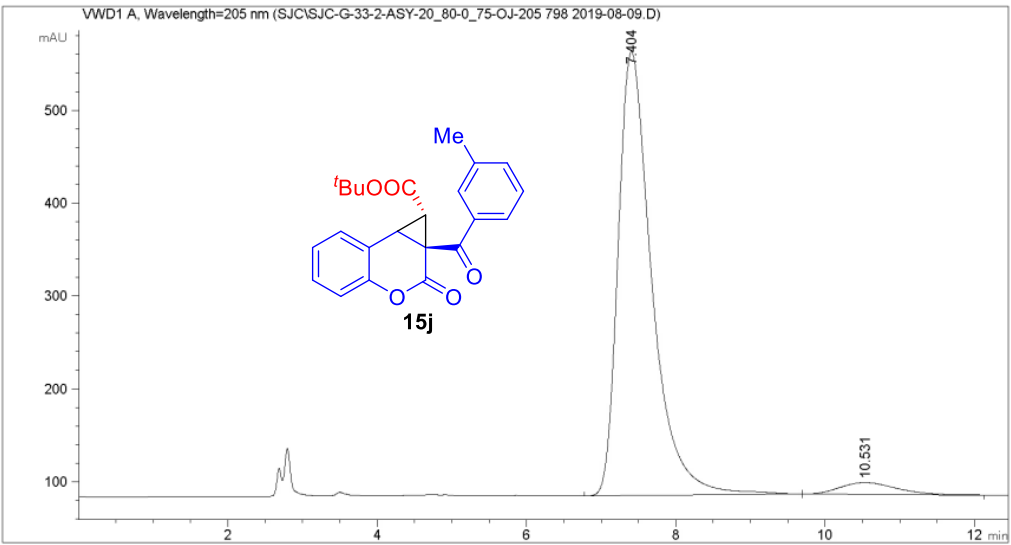

面积百分比报告

排序

它官

乘积因子

稀释因子

和稀释因子

信号 1: VWD1 A, wavelength=205 num

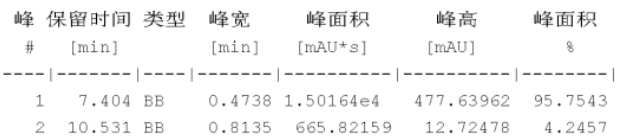

1260 2019-8-9 11:02:17 系统 


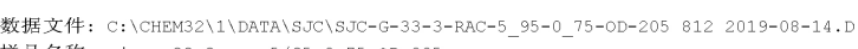
羊品名称: sic- $9-33-3-$ rac $-5 / 95-0,75-0 \mathrm{D}-205$

\begin{tabular}{|c|c|c|}
\hline 操作者 & : 系统 & \\
\hline 仪器 & $: 1260$ & 位置：样品瓶 1 \\
\hline 进样日期 & $: 2019-8-14 \quad 15: 36: 44$ & 进样量：没有进样 \\
\hline $\begin{array}{l}\text { 采集方法 } \\
\text { 最后修改 }\end{array}$ & $\begin{array}{c}: C: \backslash \text { ICHEM } 2 \backslash 1 \backslash \text { METHODS \DEF LC.M } \\
: \text { 2019-8-14 14:13:24: 系统 } \\
\text { (调用后修改) }\end{array}$ & \\
\hline $\begin{array}{l}\text { 分析方法 } \\
\text { 附加信息: }\end{array}$ & $\begin{array}{l}\text { : C: } \backslash \text { CHEM } 2 \backslash 1 \backslash \text { METHODS } \backslash D E E_{-} \text {LC.M } \\
\text { 动积分 }\end{array}$ & \\
\hline
\end{tabular}

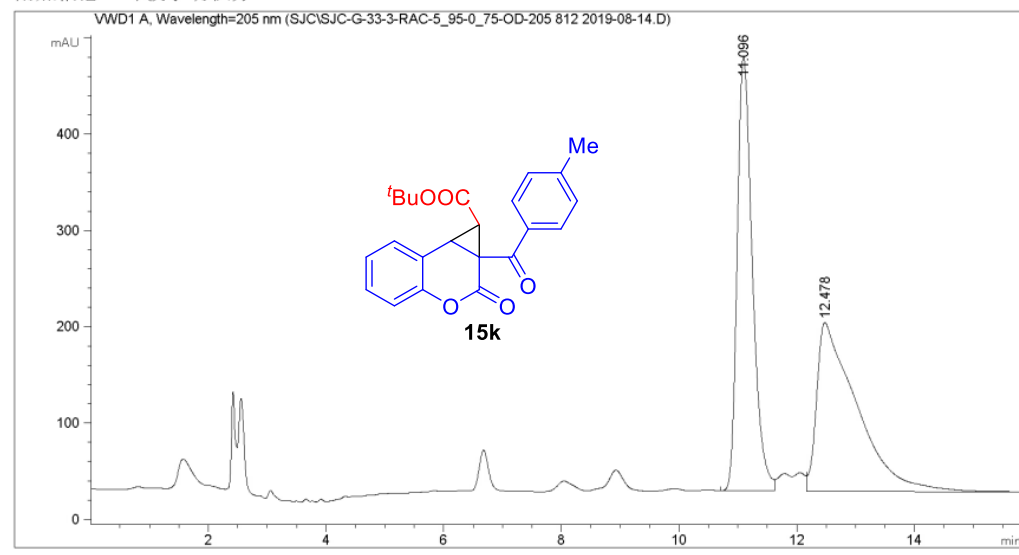

面积百分比报告

$\begin{array}{llll}\text { 排序 } & : & \text { 信号 } & \\ \text { 乘积因子 } & \vdots & 1.0000 \\ \text { 饰䉽因子 } & : & 1.0000\end{array}$

内桥称因子

信号 1: VWD1 A, wavelength=205 nm

峰保留时间类型峰宽峰面积峰高峰面积

\# $[\mathrm{min}]$
$[\mathrm{min}]$
$[\mathrm{mAU}+\mathrm{s}]$

$\begin{array}{llllll}1 & 11.096 \mathrm{BV} & 0.2743 & 8081.63477 & 450.45944 & 49.3912\end{array}$

1260 2019-8-21 14:29:06 系统

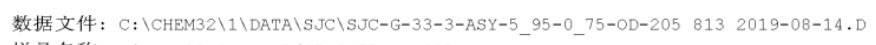

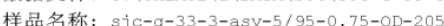

\begin{tabular}{|c|c|c|}
\hline \multicolumn{3}{|c|}{ : 系统 } \\
\hline 仪器 & $: 1260$ & 位置：样品瓶 1 \\
\hline 进样日期 & $: 2019-8-14 \quad 15: 54: 29$ & \\
\hline 采集方法 & $: C: \backslash C H E M 32 \backslash 1 \backslash M E T H O D S \backslash D E F \_L C . M$ & \\
\hline 最后修改 & $\begin{array}{c}\text { : 2019-8-14 14:13:24： 系统 } \\
\text { (调用后修改) }\end{array}$ & \\
\hline 分析方法 & $: C: \backslash C H E M 32 \backslash 1 \backslash$ METHODS $\backslash D E F$ LCC.M & \\
\hline
\end{tabular}

分析方法 $:$ : : \CHEM32\1 1 METHODS $\backslash$ DEF_LC.

附加信息: 峰被手动积分

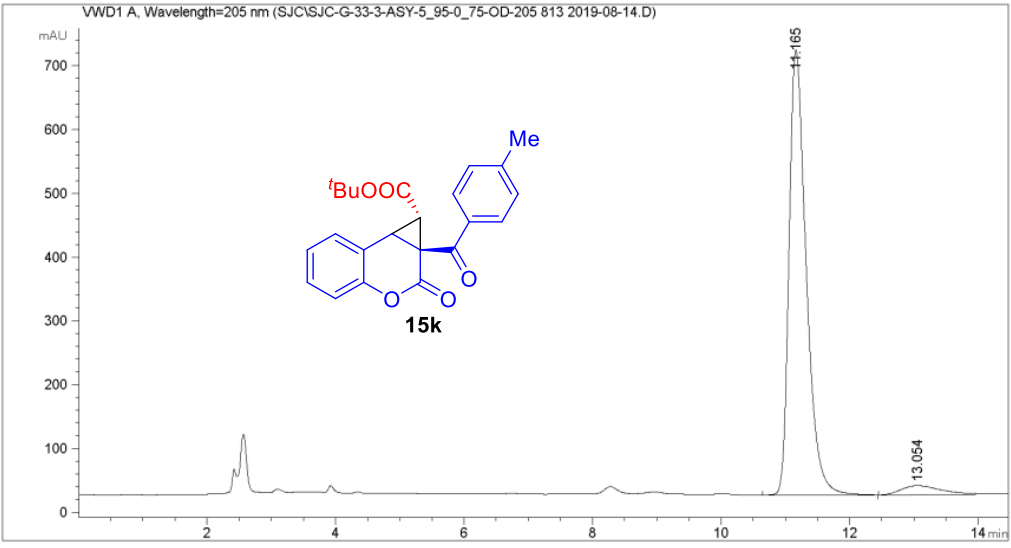

面积百分比报告

排序

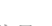

乘积因子

希释因子

乘积因子和船释因子

信号 1: VWD1 A, wavelength=205 num

峰保留时间类型峰宽峰面积峰高峰面积

$\#[\mathrm{~min}]$
$-\mathrm{min}]$$[\mathrm{mAU}+\mathrm{s}]$

$\begin{array}{llllll}1 & 11.165 \mathrm{BB} & 0.2833 & 1.29218 \mathrm{eq} & 697.03363 & 95.4121 \\ 2 & 13.054 \mathrm{BBA} & 0.6632 & 621.34937 & 14.15372 & 4.5879\end{array}$

1260 2019-8-14 16:10:47 系统 


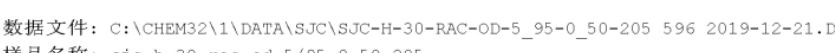
羊品名称: sjc-h-30-rac-od-5/95-0.50-20

\begin{tabular}{|c|c|c|}
\hline \multirow{3}{*}{$\begin{array}{l}\text { 操作者 } \\
\text { 仪器日期 } \\
\text { 进样日期 }\end{array}$} & : 系统 & \\
\hline & $: 1260$ & 位置：样品瓶 1 \\
\hline & $: 2019-12-21 \quad 20: 54: 24$ & \\
\hline 采集方法 & : C: \CHEM32\1\METHODS \DEF LC.M & \\
\hline 最后修改 & $\begin{array}{c}: \text { 2019-12-21 20:15:29：系统 } \\
\text { (调用后修改) }\end{array}$ & \\
\hline 折方法 & 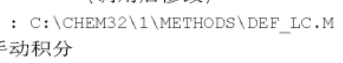 & \\
\hline
\end{tabular}

附加信息: 峰被手动积分

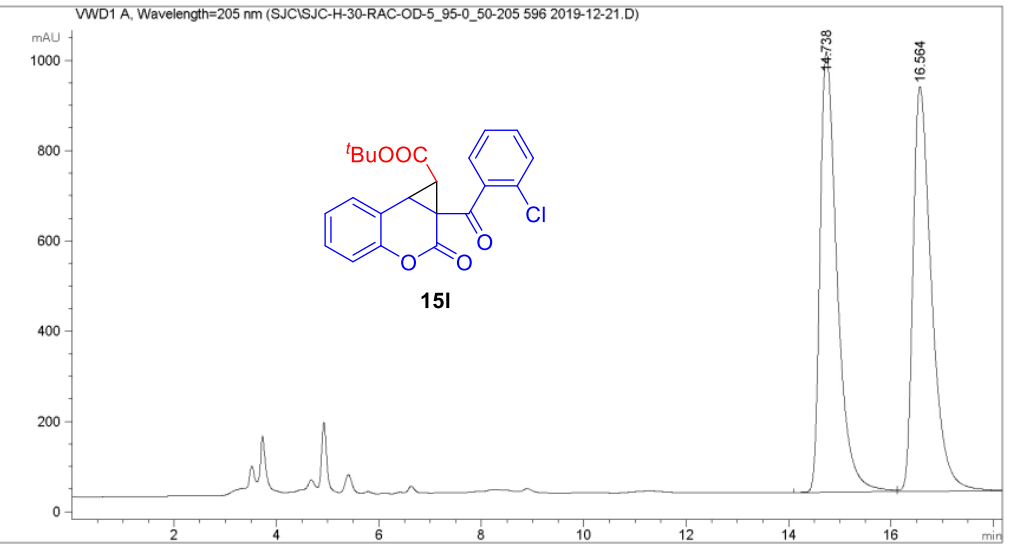

面积百分比报告

$\begin{array}{llll}\text { 排序 } & : & \text { 信号 } & \\ \text { 乘积因子 } & \vdots & 1.0000 \\ \text { 饰䉽因子 } & : & 1.0000\end{array}$

内标称因围乘积因子和顽释因子

信号 1: VWD1 A, Wavelength=205 nm

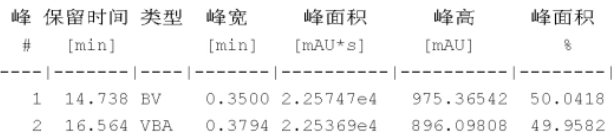

数据文件: C: \CHEM32\1\DATA $S$ S C \SJC-H-30-ASY-OD-5_95-0_50-205 597 2019-12-21.D 样品名称: sjc-h-30-asy-od-5/95-0.50-20

\begin{tabular}{|c|c|c|}
\hline \multirow{3}{*}{$\begin{array}{l}\text { 操作者 } \\
\text { 仪器 } \\
\text { 进样日期 }\end{array}$} & : 系统 & \\
\hline & $\because 1260$ & 位置：样品瓶 1 \\
\hline & $: 2019-12-21 \quad 21: 13: 28$ & \\
\hline 采集方法 & : C: \CHEM32\1\METHODS \DEF LC.M & \\
\hline 最后修改 & $\begin{array}{c}: 2019-12-21 \text { 20:15:29: 系统 } \\
\text { (调用后修改) }\end{array}$ & \\
\hline $\begin{array}{l}\text { 分析方法 } \\
\text { 附加信息: }\end{array}$ & 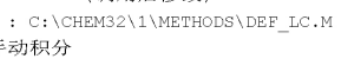 & \\
\hline
\end{tabular}

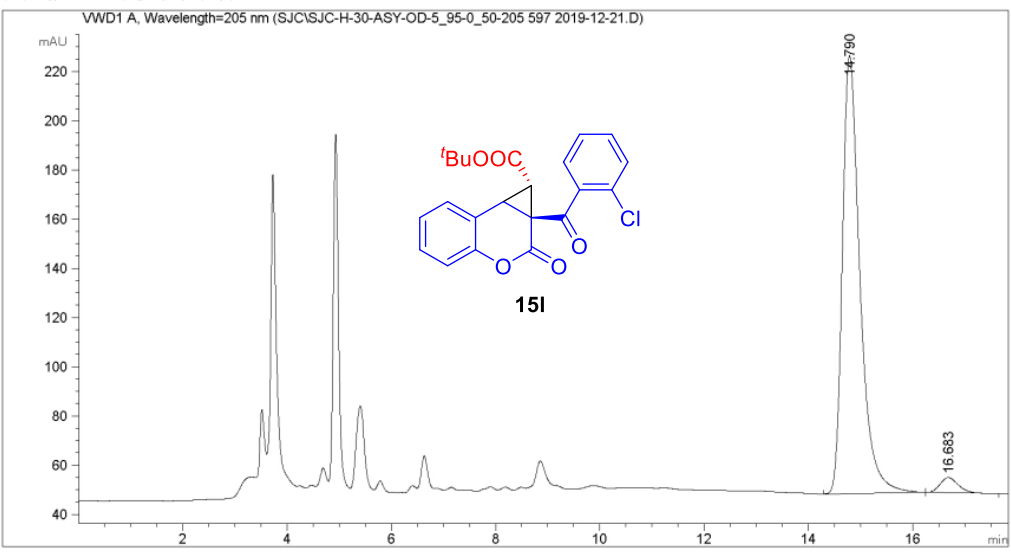

面积百分比报告

排序

(1)

乘积因子

稀释因子 : 1.0000

和矮释因子

信号 1: VWD1 A, wavelength=205 $\mathrm{nm}$

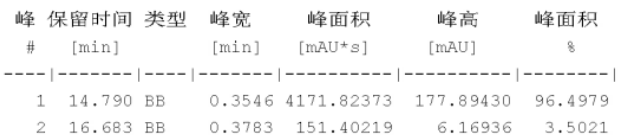

1260 2019-12-23 16:58:25 系统 


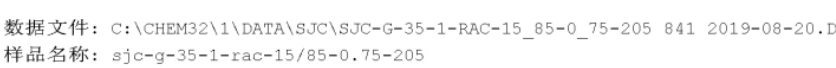

\begin{tabular}{|c|c|c|}
\hline 操作者 & : 系统 & \\
\hline 仪器 & $: 1260$ & 位置：样品瓶 1 \\
\hline 进样日期 & $: 2019-8-20 \quad 15: 20: 34$ & 进样量：没有进样 \\
\hline $\begin{array}{l}\text { 采集方法 } \\
\text { 最后修改 }\end{array}$ & $\begin{array}{l}\text { : C: \CHEM } 2 \backslash 1 \text { 1METHODS \DEF_LC.M } \\
: \text { 2019-8-20 15:09:30: 系统 } \\
\text { (调用后修改) }\end{array}$ & \\
\hline 析方法 & 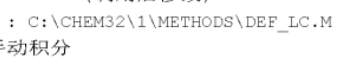 & \\
\hline
\end{tabular}

附加信息：峰被手动积分

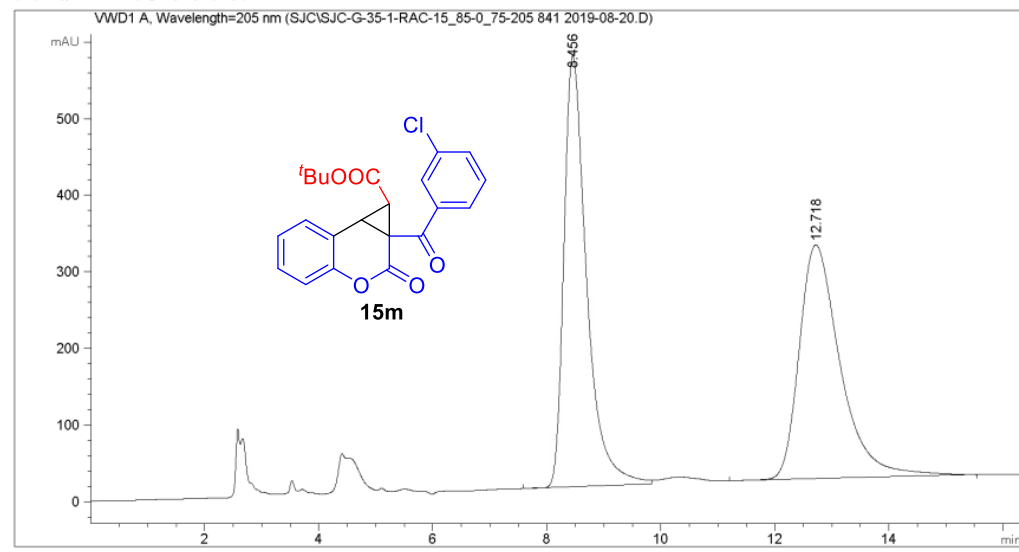

面积百分比报告

$\begin{array}{llll}\text { 排序 } & : & \text { 信号 } & \\ \text { 乘积因子 } & \vdots & 1.0000 \\ \text { 秝释因子 } & : & 1.0000\end{array}$

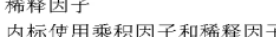

信号 1: VWD1 A, wavelength=205 nm

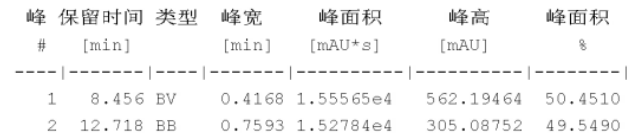

数据文件: C: \CHEM32\1\DATA $S$ S C \SJC-G-35-1-ASY-15_85-0_75-205 841 2019-08-20.D 样品名称: $s j \mathrm{j}-\mathrm{g}-35-1-2 \mathrm{sy}-15 / 85-0.75-205$

\begin{tabular}{|c|c|c|}
\hline 操作者 & : 素统 & \\
\hline 仪器 & $: 1260$ & 位置：样品瓶 1 \\
\hline 进样日期 & $: 2019-8-20 \quad 16: 41: 49$ & \\
\hline 采集方法 & 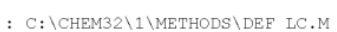 & \\
\hline 最后修改 & $\begin{array}{c}\text { :2019-8-20 16:30:54： 系统 } \\
\text { (调用后修改) }\end{array}$ & \\
\hline 析方法 & 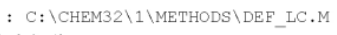 & \\
\hline
\end{tabular}

分析方法 $:$ : : \CHEM32\1 1 METHODS $\backslash$ DEF_LC.

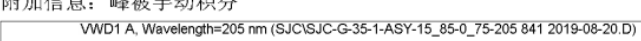

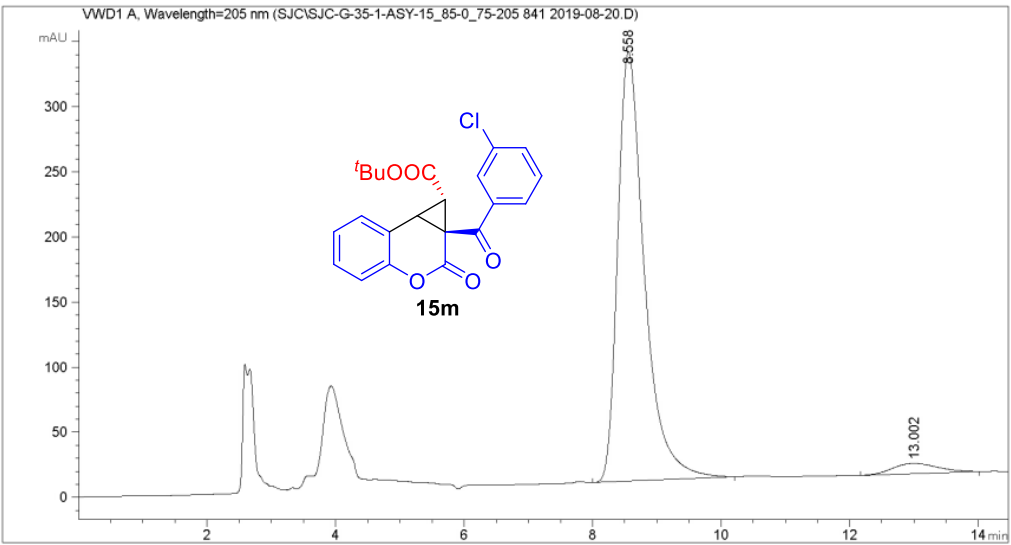

面积百分比报告

排序

它官

乘积因子

希释因子

和用乘积因子穫因子

信号 1: VWD1 A, wavelength=205 num

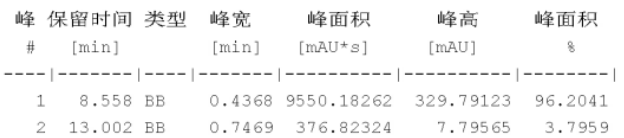

$1260 \quad 2019-8-20 \quad 16: 59: 08$ 系统 


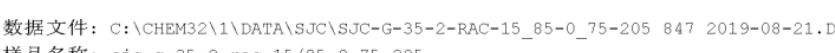
羊品名称: sjc-g-35-2-rac-15/85-0. 75-20

\begin{tabular}{|c|c|c|}
\hline 操作者 & : 系统 & \\
\hline 仪器 & $: 1260$ & 位置：样品瓶 1 \\
\hline 进样日期 & $: 2019-8-21 \quad 9: 36: 54$ & 进样量：没有进样 \\
\hline $\begin{array}{l}\text { 采集方法 } \\
\text { 最后修改 }\end{array}$ & $\begin{array}{l}\text { : C: \CHEM32\1 MMETHODS \DEFELC.M } \\
: \text { 2019-8-21 9:16:22: 系统 } \\
\text { (调用后修改) }\end{array}$ & \\
\hline $\begin{array}{l}\text { 分析方法 } \\
\text { 附加信息: }\end{array}$ & $\begin{array}{l}: \text { : }: \backslash \text { CHEM } 2 \backslash 1 \backslash \text { METHODS } \backslash D E E \text { _LC.M } \\
\text { 三动积分 }\end{array}$ & \\
\hline
\end{tabular}

附加信息: 峰被手动积分

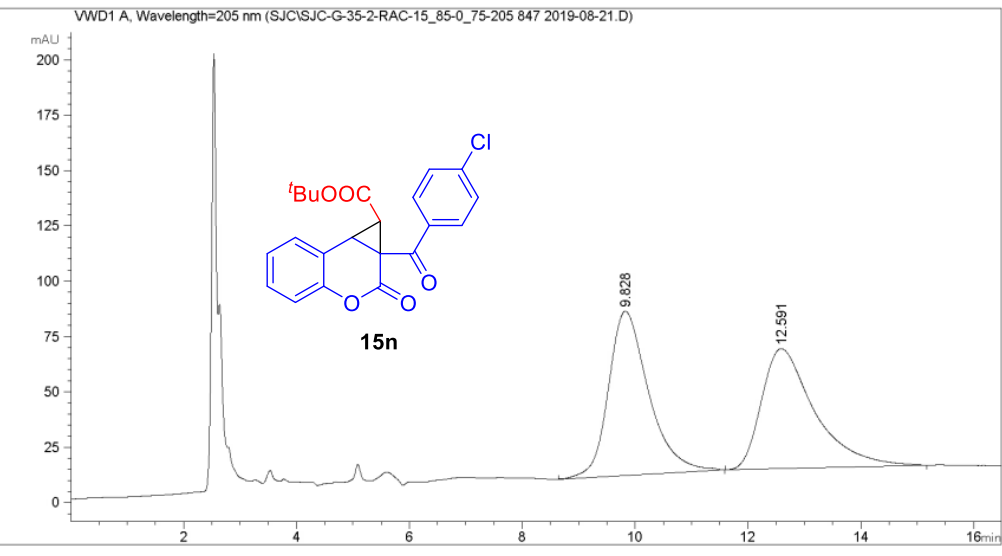

面积百分比报告

$\begin{array}{llll}\text { 排序 } & : & \text { 信号 } & \\ \text { 乘积因子 } & \vdots & 1.0000 \\ \text { 饰䉽因子 } & : & 1.0000\end{array}$

内桥称因子禹乘积因子和础轱因子

信号 1: VWD1 A, wavelength=205 nm

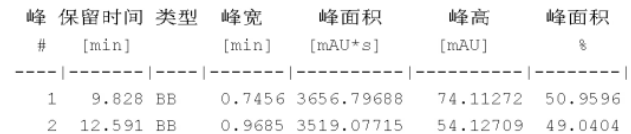

数据文件: C: \CHEM32\1\DATA $S$ S C \SJC-G-35-2-ASY-15_85-0_75-205 848 2019-08-21.D 样品名称: $s j \mathrm{c}-\mathrm{g}-35-2-a s y-15 / 85-0.75-205$

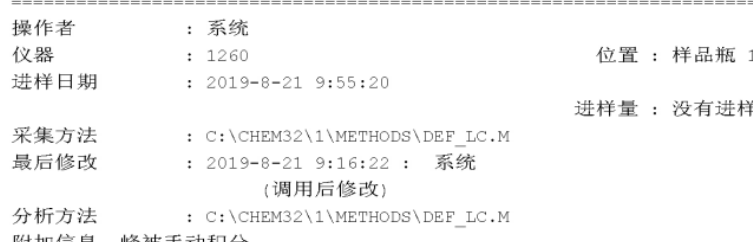

分析方法： C: \CHEM32\1\METHODS\DEF_LC.M

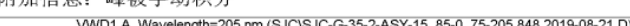

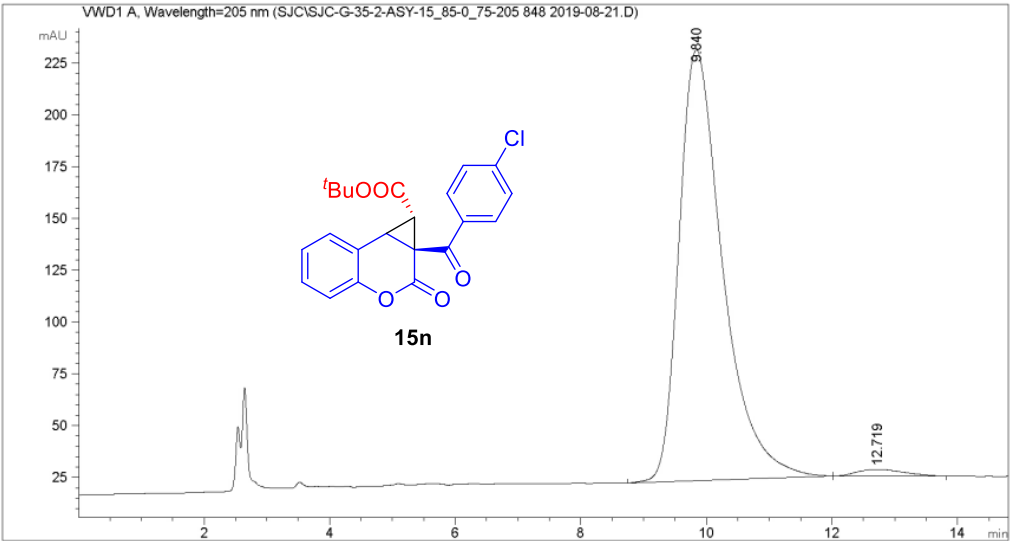

面积百分比报告

排序

信号

乘积因子

$: \quad 1.0000$

稀释因子

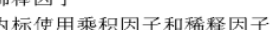

信号 1: VWD1 A, wavelength=205 nn

\begin{tabular}{|c|c|c|c|c|}
\hline $\begin{array}{l}\text { 保留时间 类型 } \\
\text { [min] }\end{array}$ & $\begin{array}{l}\text { 峰宽 } \\
\text { [min] }\end{array}$ & $\begin{array}{l}\text { 峰面积 } \\
{[\mathrm{mAU} \star s]}\end{array}$ & $\begin{array}{l}\text { 峰高 } \\
\text { [mAU] }\end{array}$ & $\begin{array}{c}\text { 峰面积 } \\
8\end{array}$ \\
\hline & 0.635 & 166.6233 & $\begin{array}{r}207.86198 \\
3.25127\end{array}$ & 1.6279 \\
\hline
\end{tabular}

1260 2019-8-21 10:50:35 系玹 


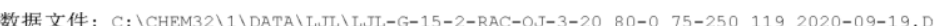
样品名称: $1 j 1-6-15-2-\mathrm{rac}-0 \mathrm{~J}-3-20 / 80-0.75-250$

\begin{tabular}{|c|c|c|}
\hline 操作者 & : 系统 & \\
\hline 仪器 & $: 1260$ & 位置：样品瓶 1 \\
\hline 进样日期 & : 2020-9-19 14:50:11 & 进样量：没有进样 \\
\hline $\begin{array}{l}\text { 采集方法 } \\
\text { 最后修改 }\end{array}$ & 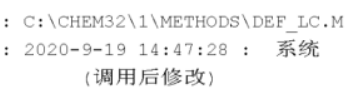 & \\
\hline 分析方法 & $: C: \backslash C H E M 32 \backslash 1 \backslash M E T H O D S \backslash D E F+L C . M$ & \\
\hline
\end{tabular}

分析方法 : C: \CHEM32\1 \METHODS \DEF_LC.M 附加信息: 峰被手动积分

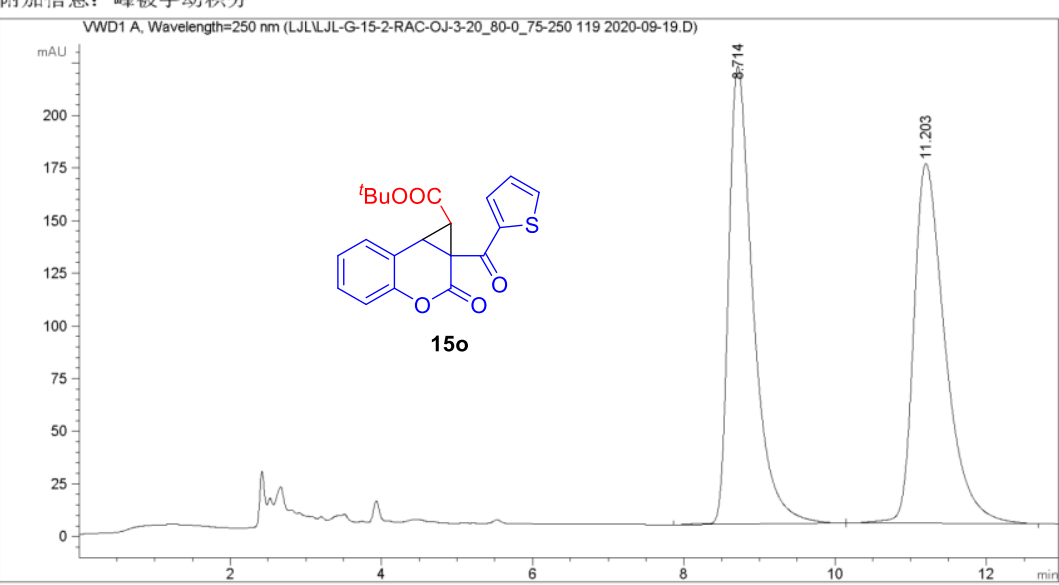

面积百分比报告

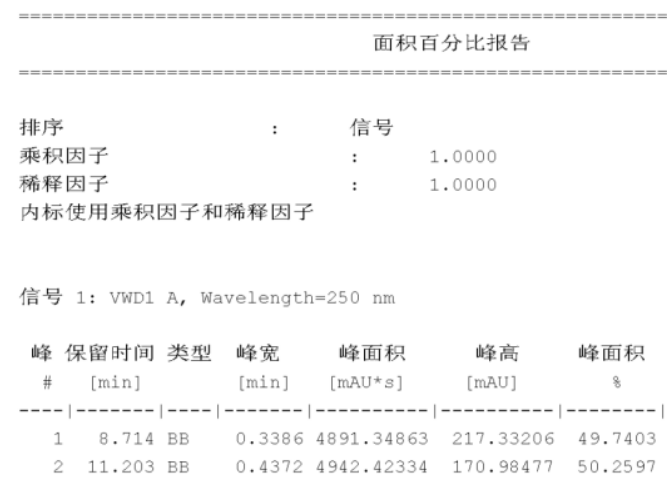

\begin{tabular}{|c|c|c|}
\hline 操作者 & : 系统 & \\
\hline 仪器 & $: 1260$ & 位置：样品 \\
\hline 进样日期 & $: 2020-9-1915: 04: 42$ & 进样量：没有远 \\
\hline $\begin{array}{l}\text { 采集方法 } \\
\text { 最后修改 }\end{array}$ & 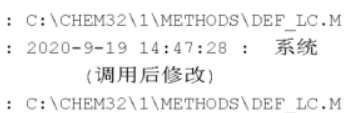 & \\
\hline
\end{tabular}

附加信息: 峰被手动积分

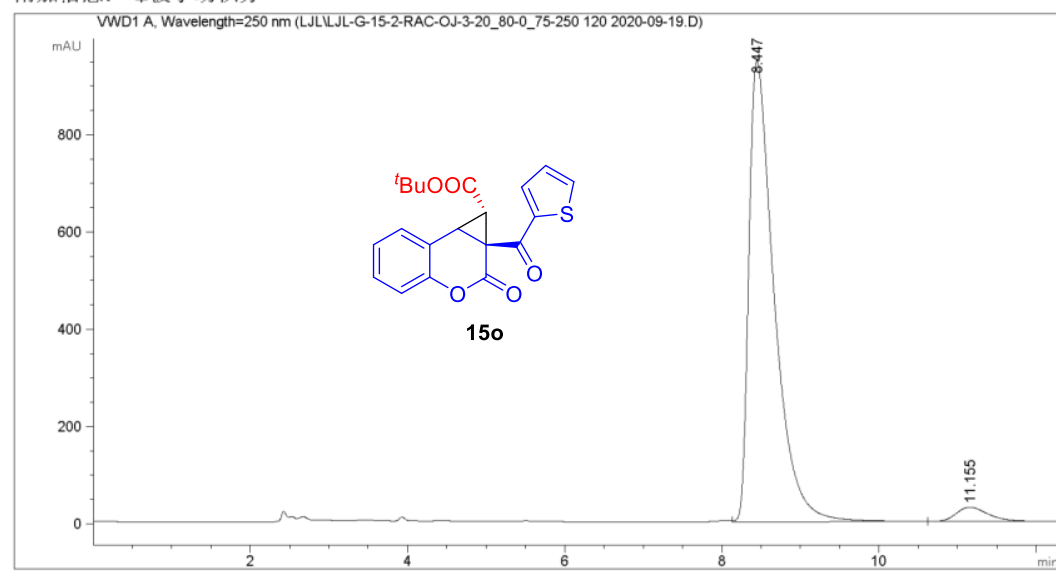

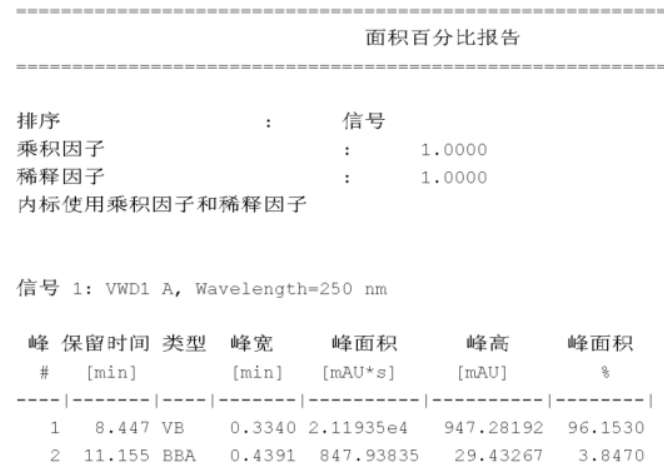




\section{HRMS report for 11 a}

Mass Spectrum List Report

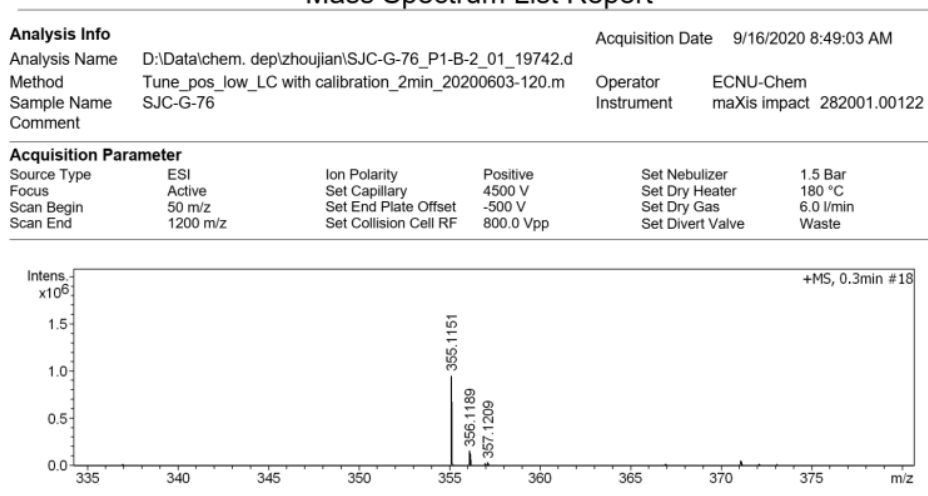

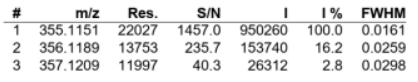

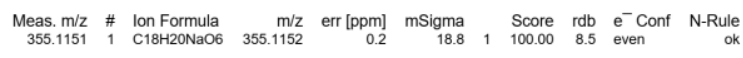

HRMS report for $\mathbf{1 1 b}$

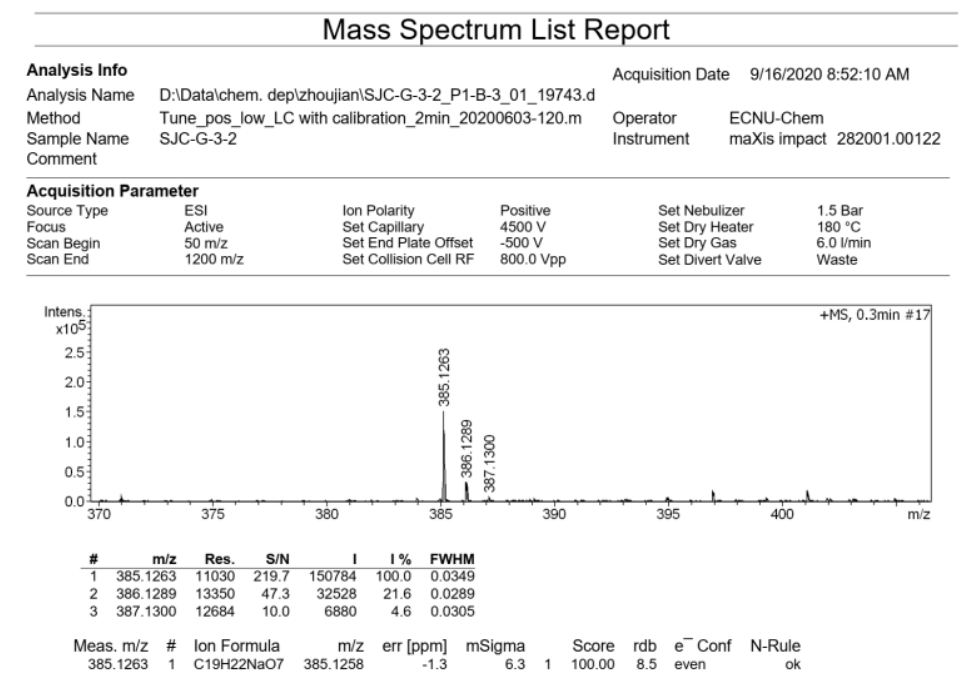

Bruker Compass DataAnalysis 4.1 printed: 9/16/2020 9:33:04 AM $\quad$ by: ECNU-Chem Page 1 of 1 
HRMS report for 11c

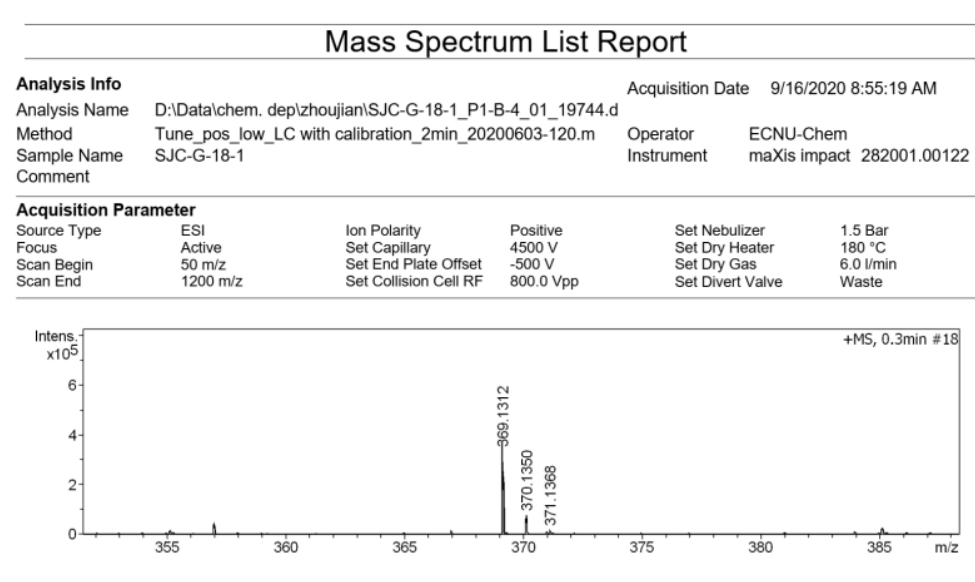

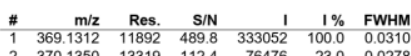

$\begin{array}{lllllll}2 & 370.1350 & 13319 & 12.4 & 76476 & 23.0 & 0.0278 \\ 3 & 371.1368 & 13385 & 20.8 & 14128 & 4.2 & 0.0277\end{array}$

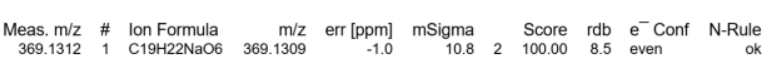

HRMS report for $\mathbf{1 1 d}$

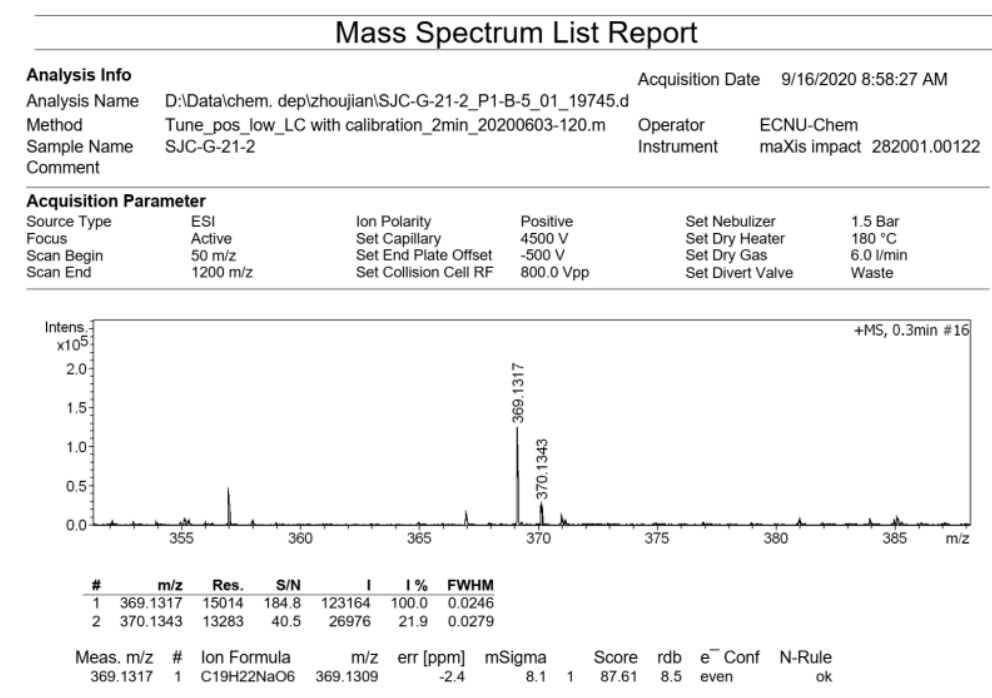

Bruker Compass DataAnalysis $4.1 \quad$ printed: 9/16/2020 9:36:21 AM $\quad$ by: ECNU-Chem Page 1 of 1 
HRMS report for 11e
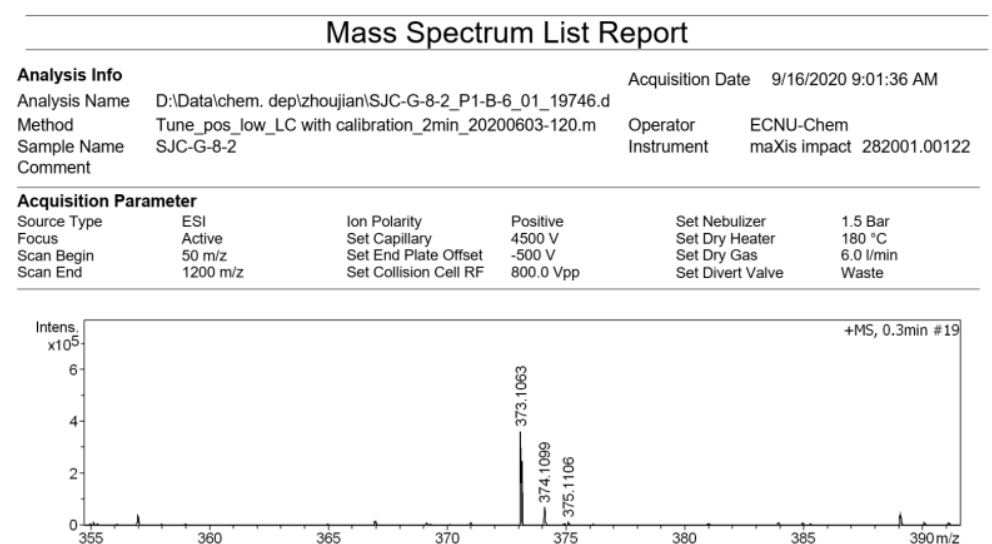

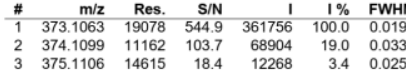

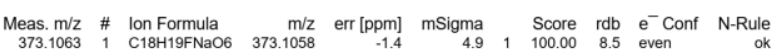

HRMS report for $\mathbf{1 1 f}$

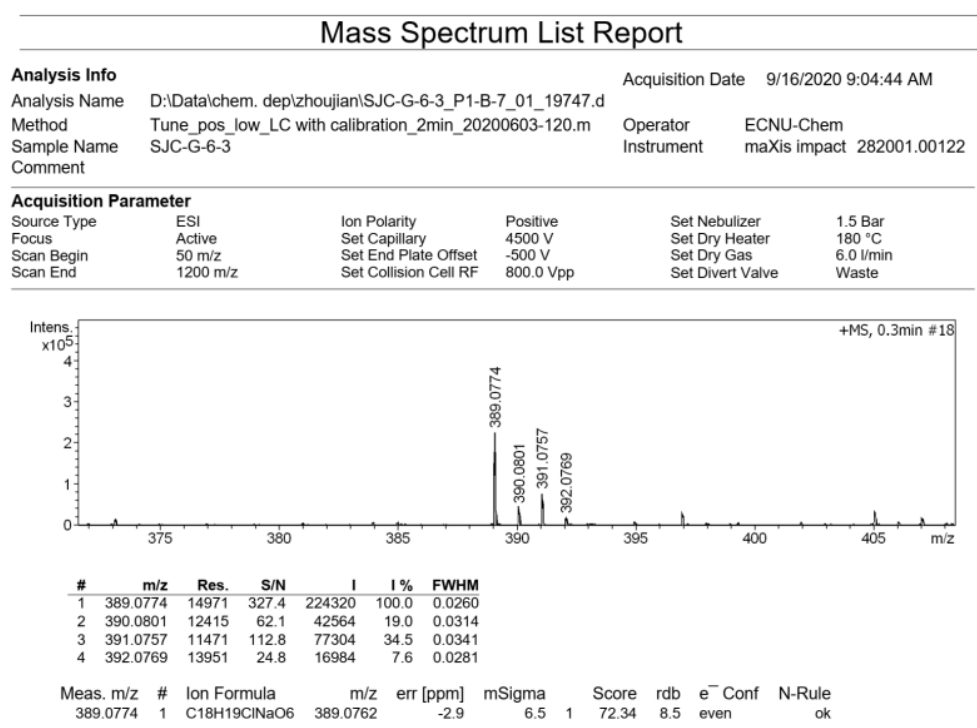

Bruker Compass DataAnalysis 4.1 printed: 9/16/2020 9:38:15 AM $\quad$ by: ECNU-Chem Page 1 of 1 
HRMS report for $\mathbf{1 1 g}$

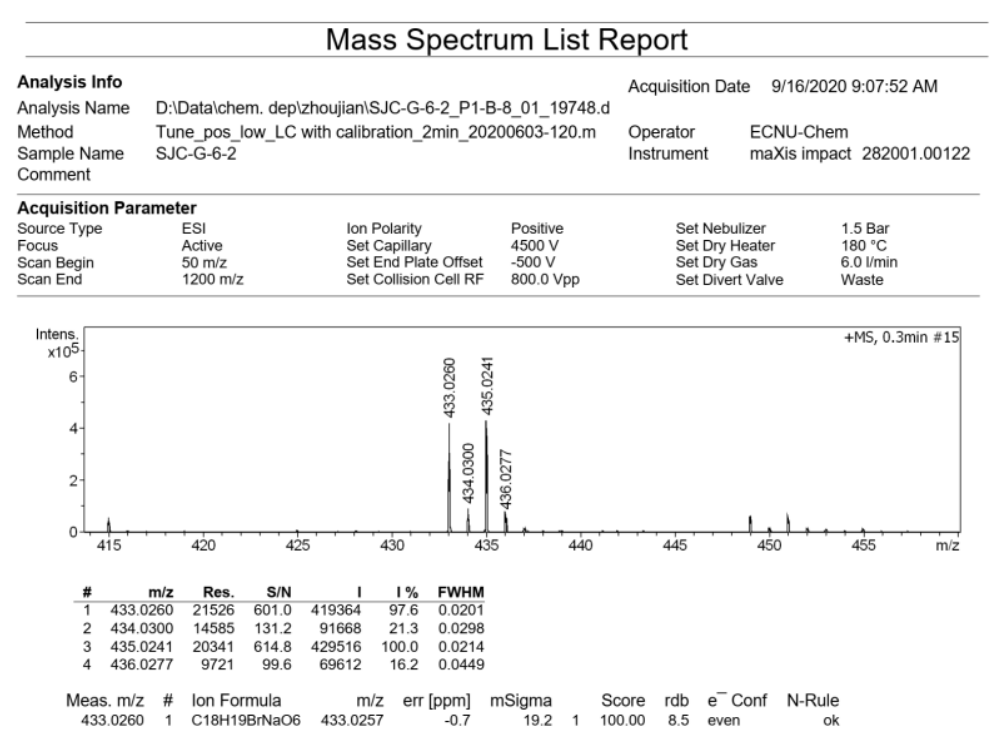

Bruker Compass DataAnalysis 4.1 printed: 9/16/2020 9:41:45 AM by: ECNU-Chem Page 1 of 1
HRMS report for $\mathbf{1 1 h}$

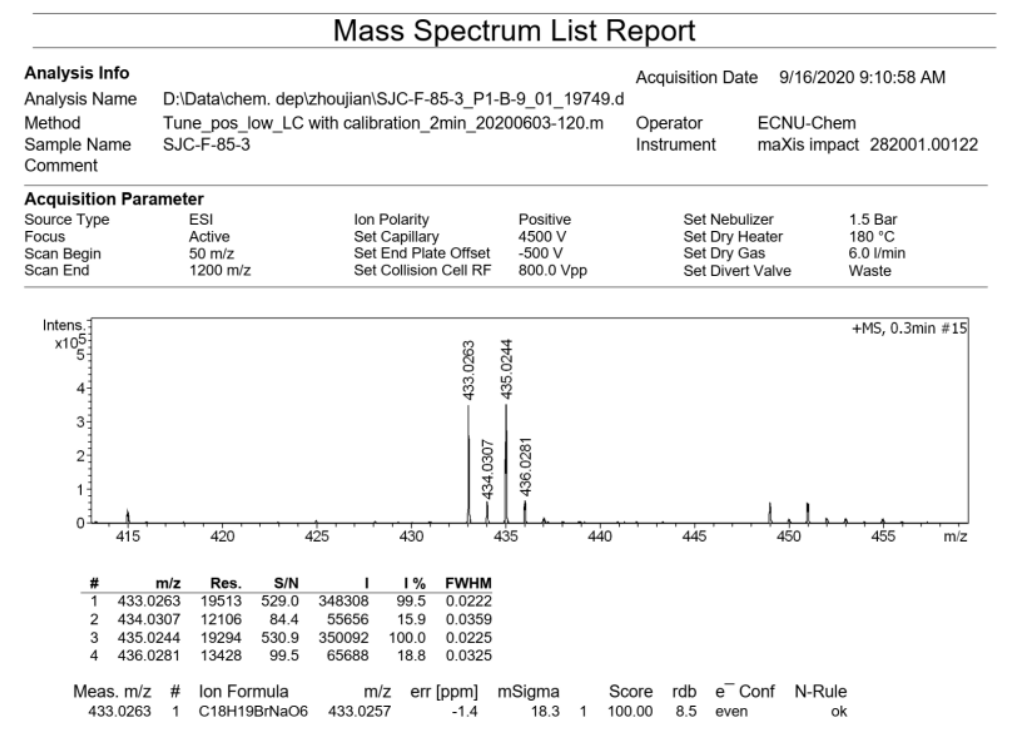

Bruker Compass DataAnalysis 4.1 printed: 9/16/2020 9:42:48 AM by: ECNU-Chem Page 1 of 1 
HRMS report for 11i

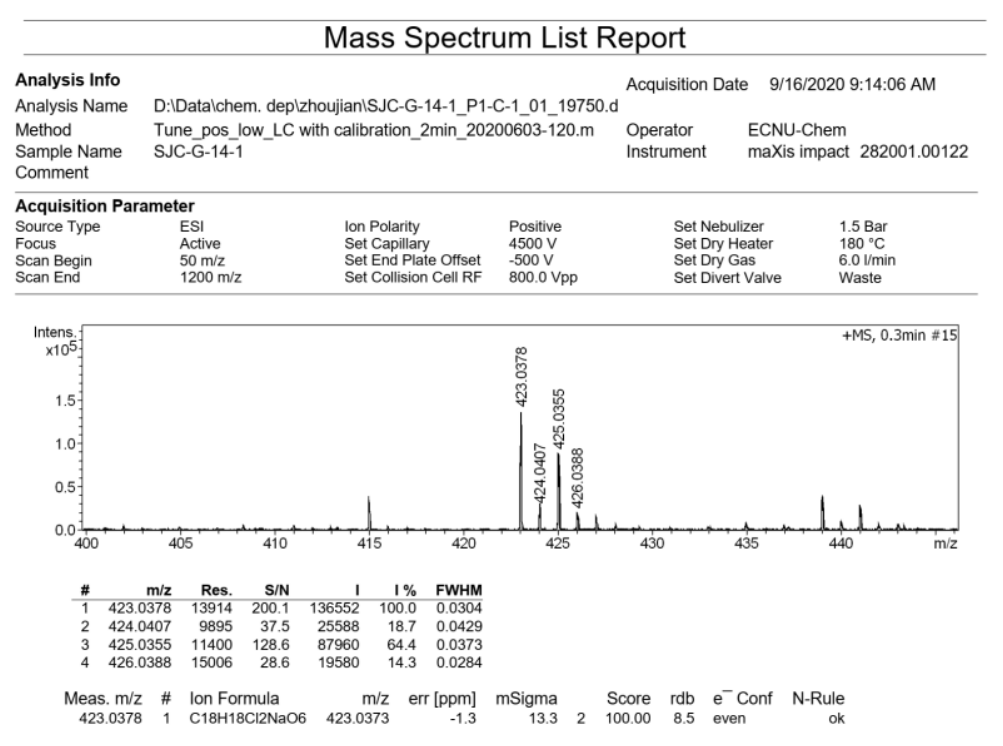

Bruker Compass DataAnalysis 4.1 printed: 9/16/2020 9:43:50 AM by: ECNU-Chem Page 1 of 1
HRMS report for $\mathbf{1 1 j}$

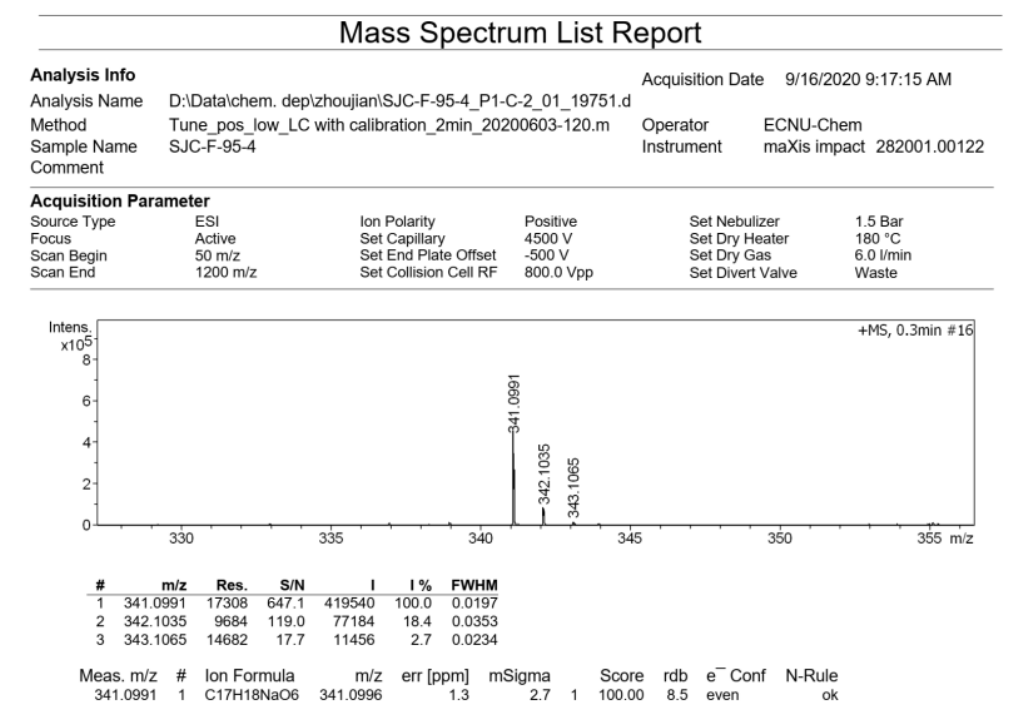

Bruker Compass DataAnalysis 4.1 printed: 9/16/2020 9:44:59 AM $\quad$ by: ECNU-Chem Page 1 of 1 
HRMS report for 11k

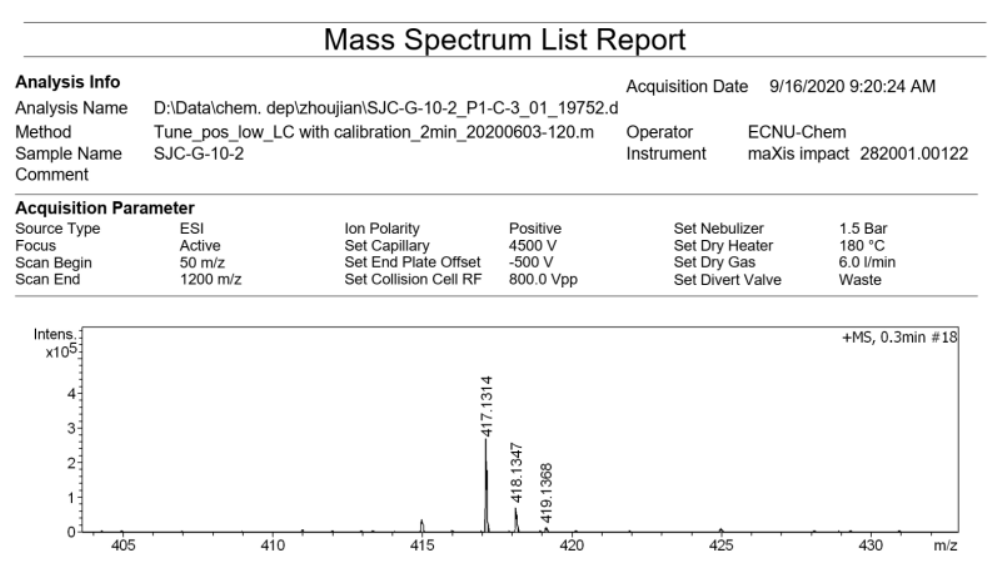

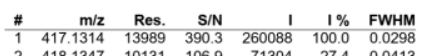

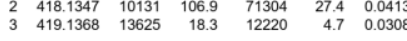

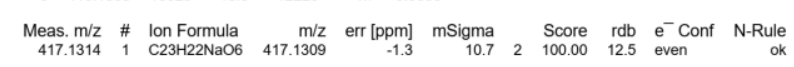

\section{HRMS report for $\mathbf{1 5 a}$}

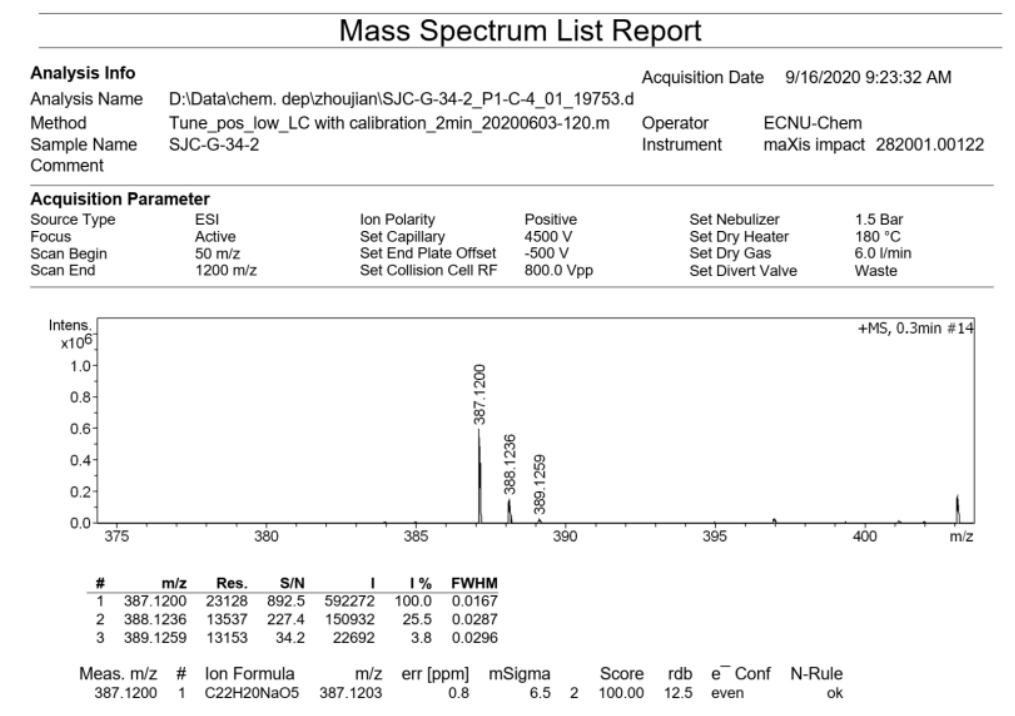

Bruker Compass DataAnalysis 4.1 printed: 9/16/2020 9:47:32 AM $\quad$ by: ECNU-Chem Page 1 of 1 
HRMS report for $\mathbf{1 5 b}$

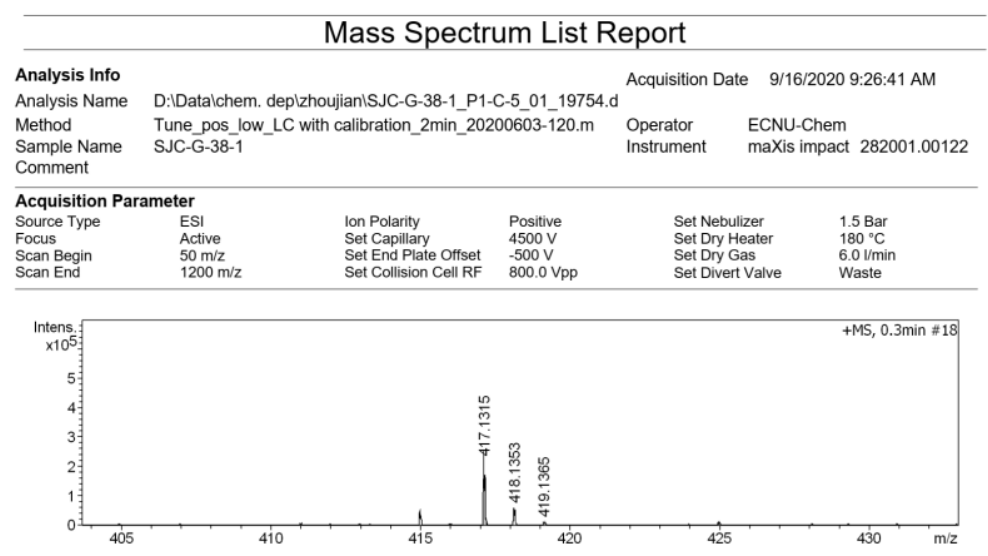

\begin{tabular}{rrrrrrr}
$\#$ & m/z & Res. & SIN & 21907 & $1 \%$ & FWHM \\
\hline 1 & 417.1315 & 16139 & 325.1 & 219076 & 100.0 & 0.0258
\end{tabular}

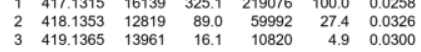

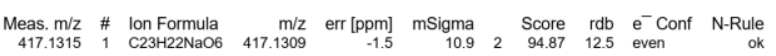

HRMS report for $\mathbf{1 5 c}$

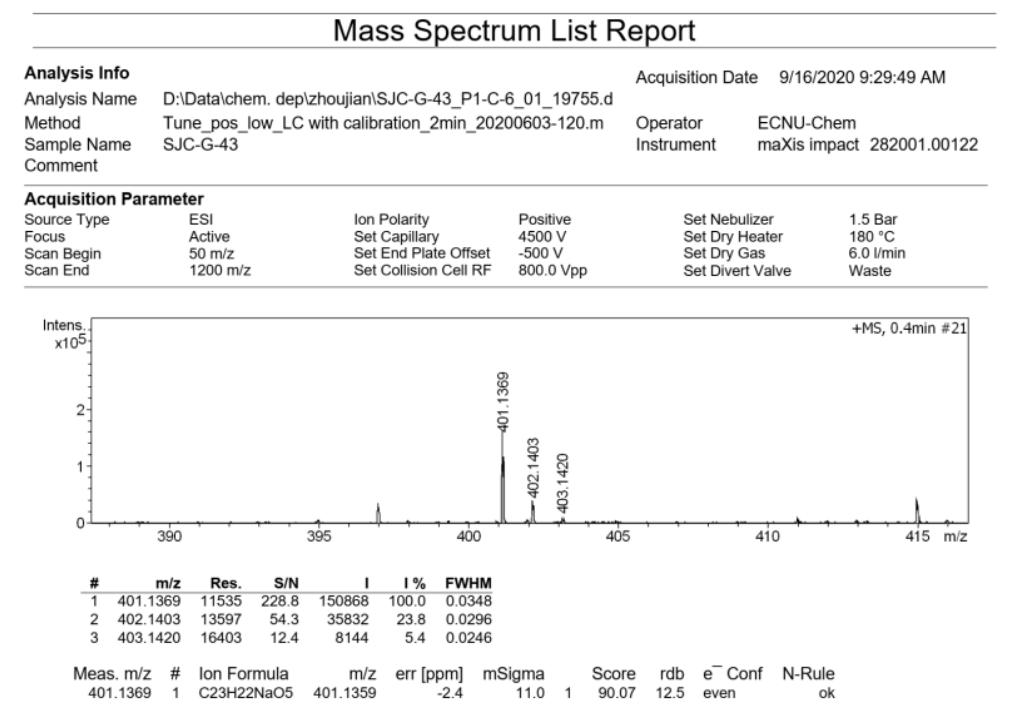

B̈ruker Compass DataAnalysis 4.1 printed: 9/16/2020 9:50:22 AM $\quad$ by: ECNU-Chem Page 1 of 1 
HRMS report for $\mathbf{1 5 d}$

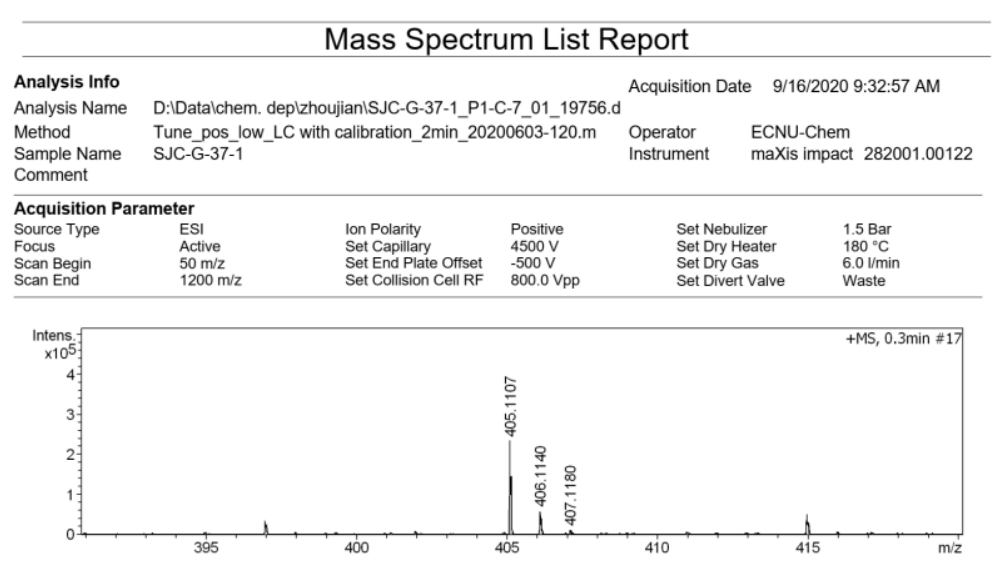

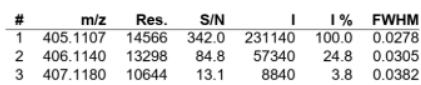

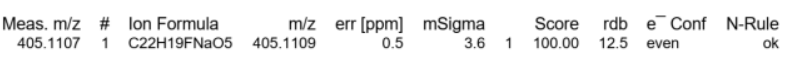

\section{HRMS report for $\mathbf{1 5 e}$}

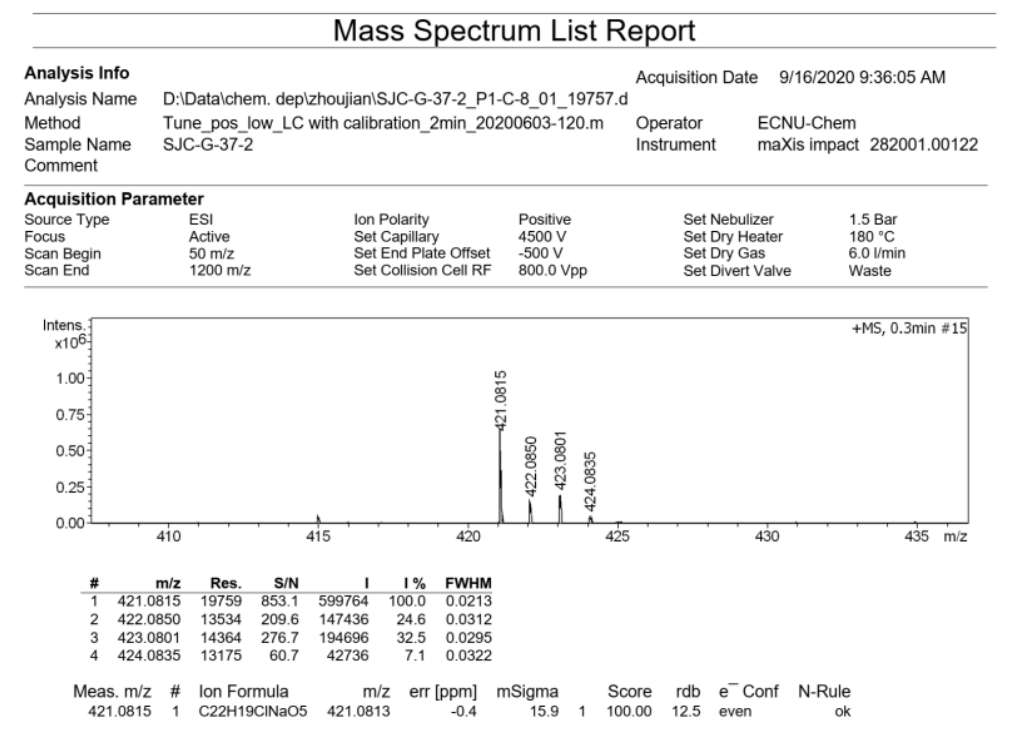

\begin{tabular}{llll}
\hline Bruker Compass DataAnalysis $4.1 \quad$ printed: 9/16/2020 9:52:36 AM $\quad$ by: ECNU-Chem Page 1 of 1
\end{tabular} 
HRMS report for $\mathbf{1 5 f}$

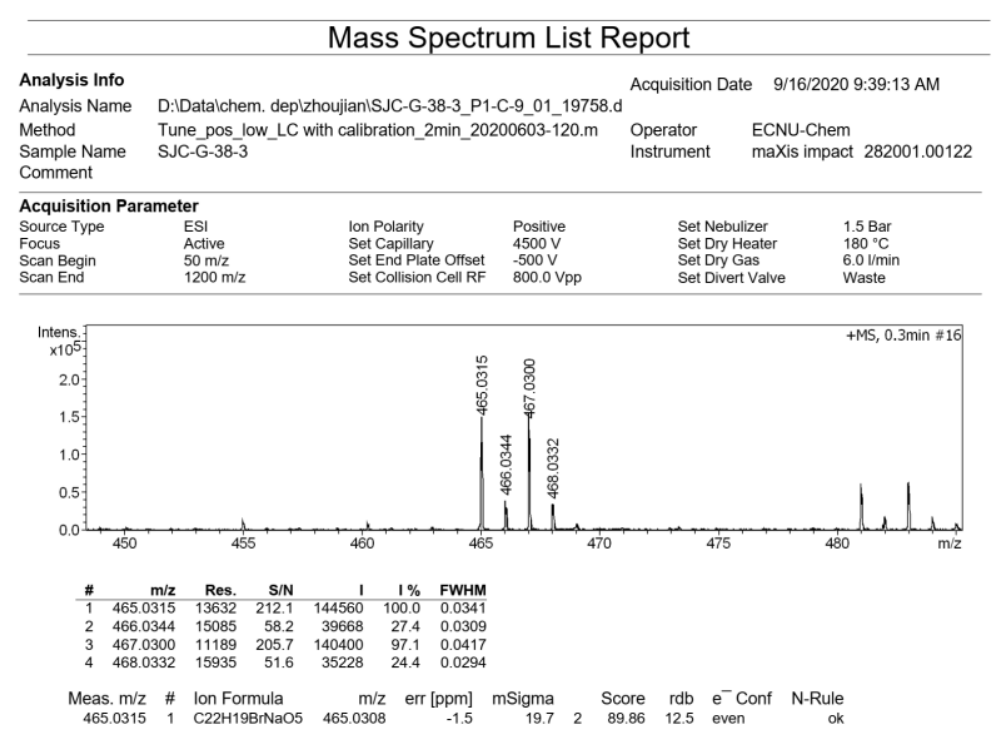

Bruker Compass DataAnalysis 4.1 printed: 9/16/2020 9:54:06 AM by: ECNU-Chem Page 1 of 1

\section{HRMS report for $\mathbf{1 5 g}$}

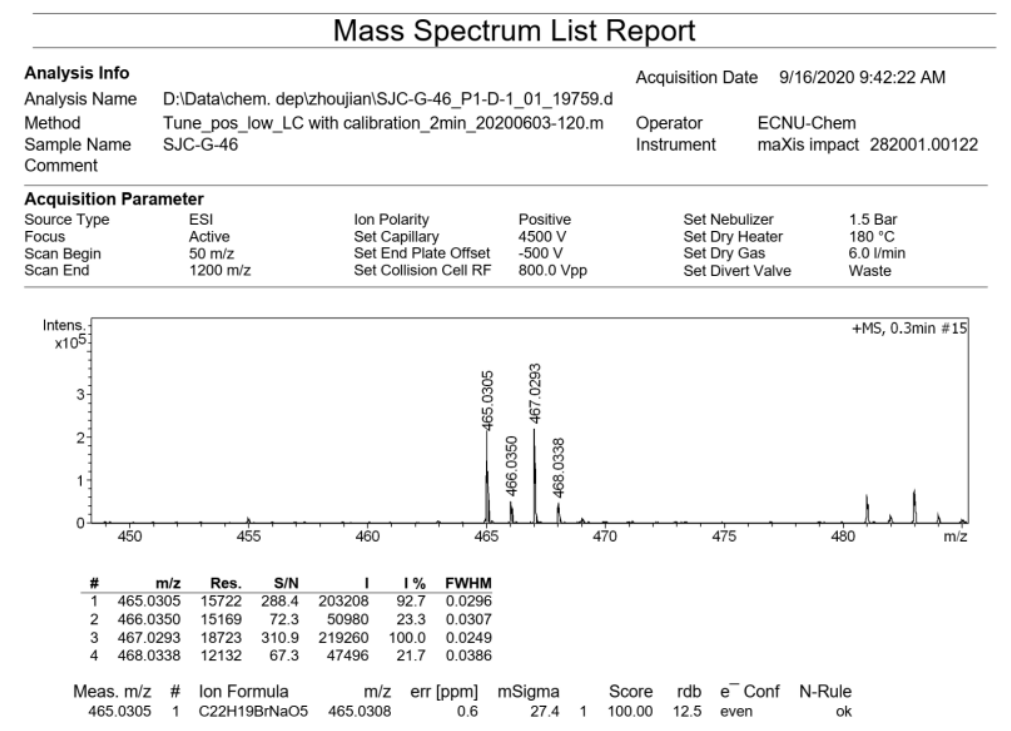

Bruker Compass DataAnalysis $4.1 \quad$ printed: 9/16/2020 9:55:02 AM $\quad$ by: ECNU-Chem Page 1 of 1 
HRMS report for $\mathbf{1 5 h}$

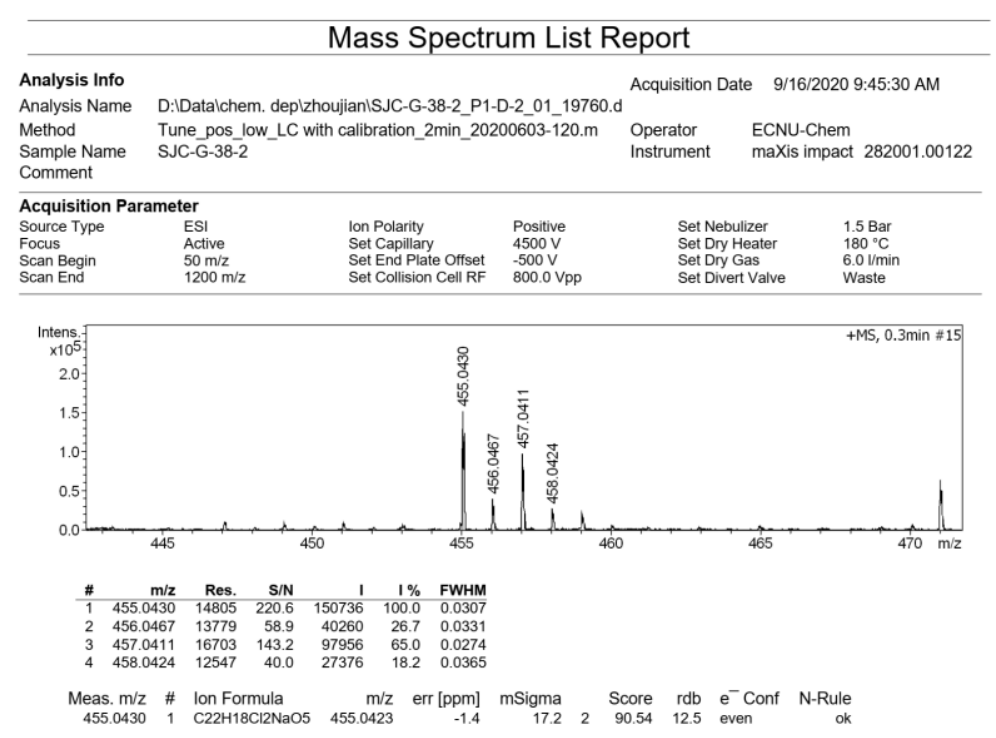

Bruker Compass DataAnalysis 4.1 printed: 9/16/2020 9:56:36 AM by: ECNU-Chem Page 1 of 1
HRMS report for $\mathbf{1 5 i}$

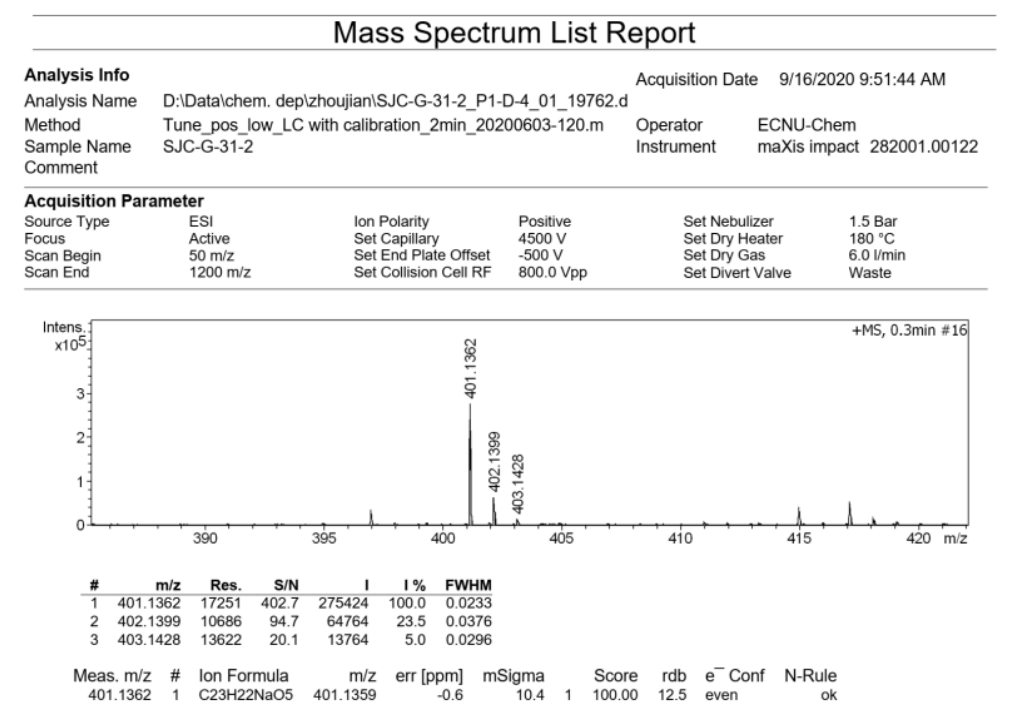

Bruker Compass DataAnalysis $4.1 \quad$ printed: 9/16/2020 9:59:43 AM $\quad$ by: ECNU-Chem Page 1 of 1 


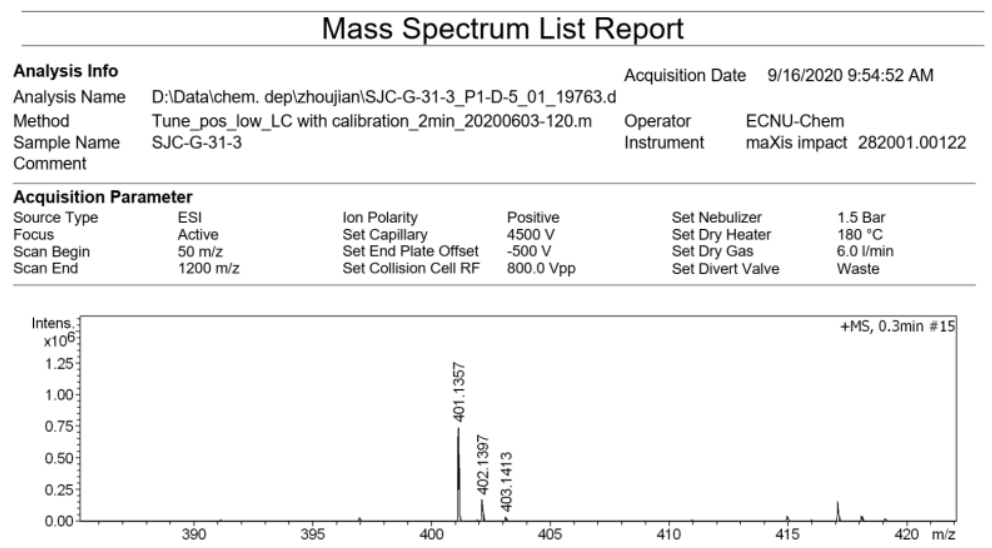

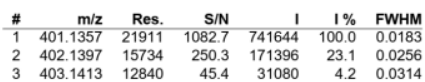

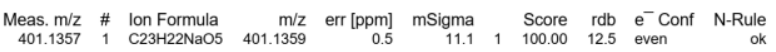

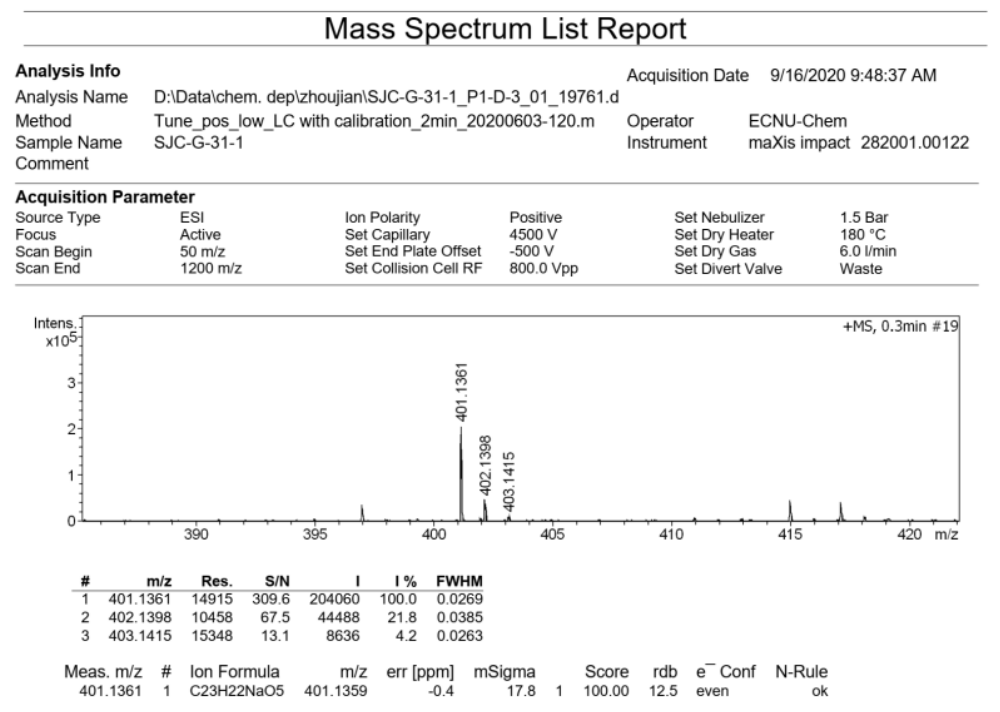

Bruker Compass DataAnalysis $4.1 \quad$ printed: 9/16/2020 9:57:41 AM $\quad$ by: ECNU-Chem Page 1 of 1 


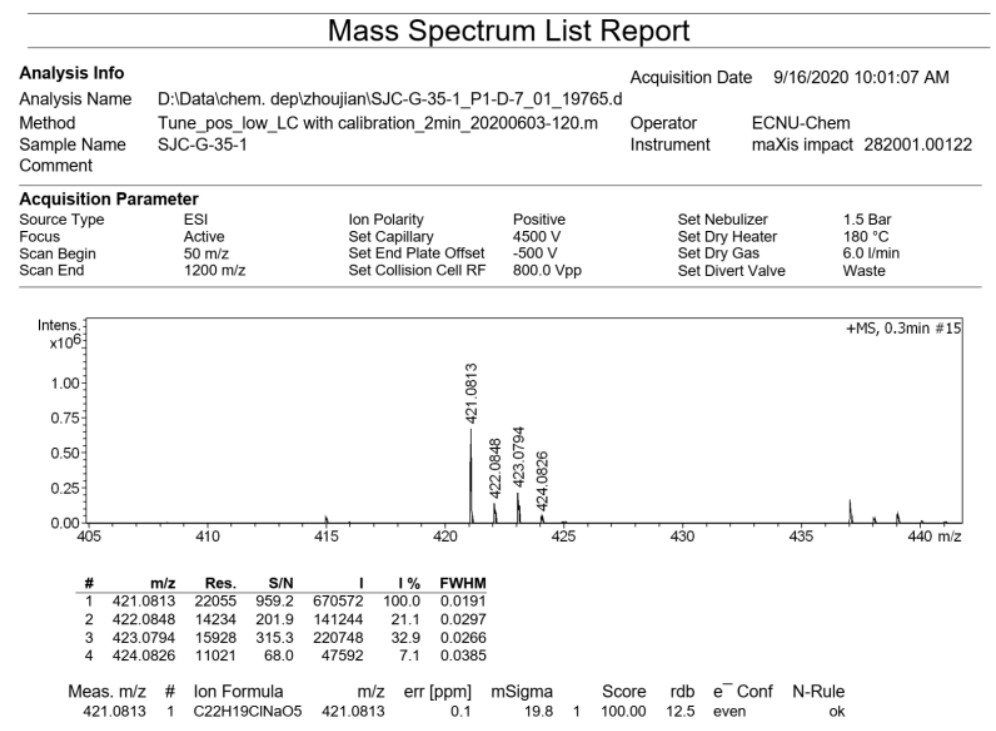

Bruker Compass DataAnalysis 4.1 printed: 9/16/2020 10:04:40 AM by: ECNU-Chem Page 1 of 1

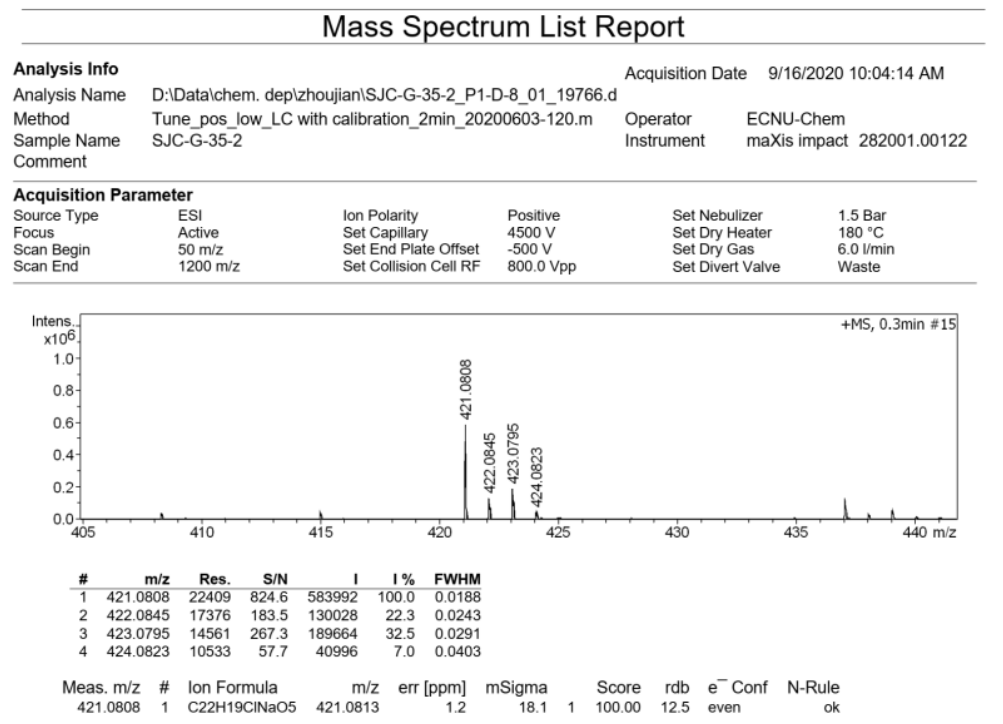

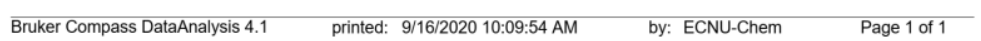


HRMS report for $\mathbf{1 5 n}$

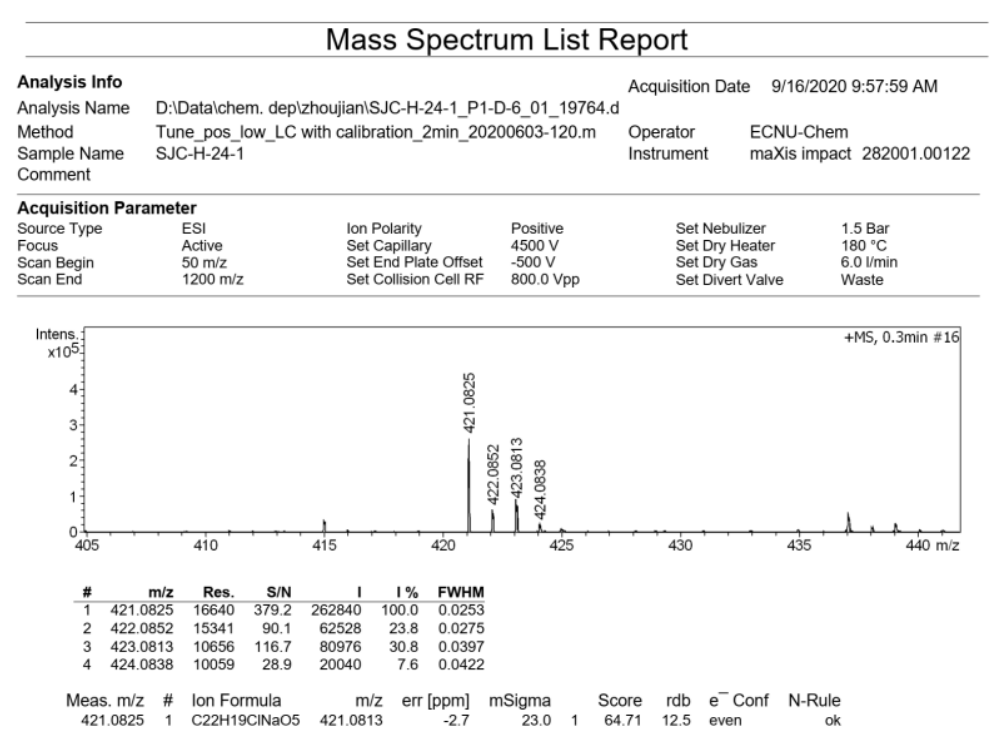

Bruker Compass DataAnalysis 4.1 printed: 9/16/2020 10:02:37 AM by: ECNU-Chem Page 1 of 1

\section{HRMS report for $\mathbf{1 5 0}$}

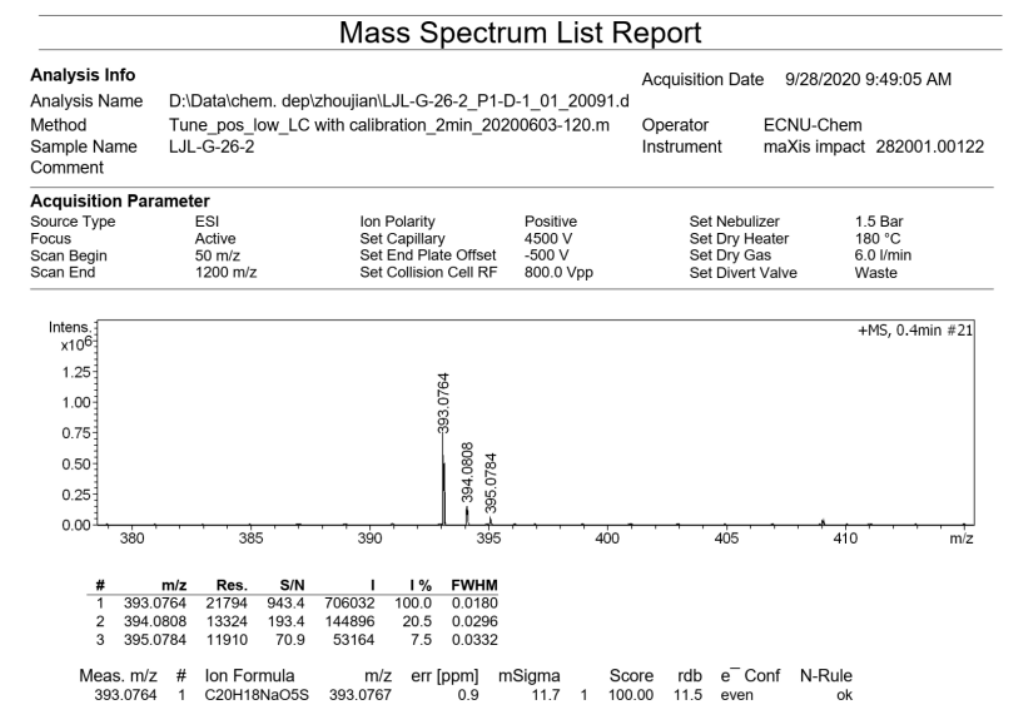

\begin{tabular}{llll}
\hline Bruker Compass DataAnalysis $4.1 \quad$ printed: 9/28/2020 10:27:15 AM & by: ECNU-Chem Page 1 of 1
\end{tabular} 